EBERHARD WILLE

KLAUS KNABNER (HRSG.)

\title{
DEZENTRALISIERUNG
} UND

FLEXIBILISIERUNG IM

GESUNDHEITSWESEN 
EBERHARD WILLE

KLAUS KNABNER (HRSG.)

\section{DEZENTRALISIERUNG UND FLEXIBILISIERUNG IM GESUNDHEITSWESEN}

Dieser Band der Bad Orber Gespräche 2010 enthält die erweiterten Referate eines interdisziplinären Workshops zum Thema „Dezentralisierung und Flexibilisierung im deutschen Gesundheitswesen“. Vertreter der Ministerien, des Bundesversicherungsamtes, der Krankenkassen, der ärztlichen Verbände, der Krankenhausträger, der pharmazeutischen Industrie und der Wissenschaft erörtern Probleme und Optionen einer Dezentralisierung der Gesundheitspolitik, der regionalen Bedarfsplanung, der Flexibilisierung in der stationären und ambulanten Versorgung, der individualisierten Medizin und der Frühbewertung neuer patentgeschützter Arzneimittel.

Eberhard Wille wurde 1942 in Berlin geboren. Nach dem Diplom-Examen 1966 an der Universität Bonn, der Promotion 1969 und der Habilitation 1973 an der Universität Mainz war er von 1975 bis 2010 Professor der Volkswirtschaftslehre und Finanzwissenschaft an der Universität Mannheim. Er ist derzeit als Emeritus u. a. Mitglied des Wissenschaftlichen Beirats beim Bundesministerium für Wirtschaft und Technologie sowie Mitglied und seit 2002 Vorsitzender des Sachverständigenrates für die Begutachtung der Entwicklung im Gesundheitswesen.

Klaus Knabner wurde 1944 in Bramsche geboren. Er studierte Betriebswirtschaftslehre und Volkswirtschaftslehre und promovierte an der Freien Universität Berlin. Bei einem deutschen Pharmaunternehmen war er Leiter der Abteilung Gesundheitswesen und Kommunikation und ist derzeit Lehrbeauftragter für Krankenhausmanagement für verschiedene Institutionen. 
Dezentralisierung und Flexibilisierung im Gesundheitswesen 


\section{ALLOKATION IM MARKTWIRTSCHAFTLICHEN SYSTEM \\ Herausgegeben von \\ Heinz König (†), Hans-Heinrich Nachtkamp, Ulrich Schlieper, Eberhard Wille}

\section{Band 64}

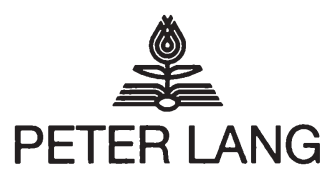

Frankfurt am Main · Berlin · Bern · Bruxelles · New York · Oxford · Wien 


\section{EBERHARD WILLE \\ KLAUS KNABNER \\ (HRSG.)}

\section{DEZENTRALISIERUNG UND FLEXIBILISIERUNG IM GESUNDHEITSWESEN \\ 15. Bad Orber Gespräche über kontroverse Themen im Gesundheitswesen 18.-19. November 2010}

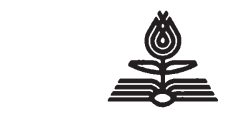

PETER LANG

Internationaler Verlag der Wissenschaften 
Bibliografische Information der Deutschen Nationalbibliothek

Die Deutsche Nationalbibliothek verzeichnet diese Publikation in der Deutschen Nationalbibliografie; detaillierte bibliografische Daten sind im Internet über http://dnb.d-nb.de abrufbar.

Open Access: The online version of this publication is published on www.peterlang.com and www.econstor.eu under the international Creative Commons License CC-BY 4.0. Learn more on how you can use and share this work: http://creativecommons. org/licenses/by/4.0.

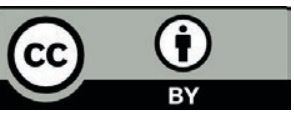

This book is available Open Access thanks to the kind support of ZBW - Leibniz-Informationszentrum Wirtschaft.

\author{
Umschlaggestaltung: \\ Atelier Platen, Friedberg
}

Gedruckt auf alterungsbeständigem, säurefreiem Papier.

\author{
ISSN 0939-7728 \\ ISBN 978-3-631-63539-1 \\ ISBN 978-3-631-75550-1 (eBook) \\ (C) Peter Lang GmbH \\ Internationaler Verlag der Wissenschaften \\ Frankfurt am Main 2011 \\ Alle Rechte vorbehalten.
}

Das Werk einschließlich aller seiner Teile ist urheberrechtlich geschützt. Jede Verwertung außerhalb der engen Grenzen des Urheberrechtsgesetzes ist ohne Zustimmung des Verlages unzulässig und strafbar. Das gilt insbesondere für Vervielfältigungen, Übersetzungen, Mikroverfilmungen und die Einspeicherung und Verarbeitung in elektronischen Systemen.

www.peterlang.de 


\section{Inhaltsverzeichnis}

Klaus Knabner

Begrüßung......

Christian Weber

Ansatzpunkte einer Dezentralisierung in der Gesundheitspolitik

Günter Neubauer

Ziele und Optionen einer Dezentralisierung...

\section{Manfred Zach}

Zum Stellenwert dezentraler Lösungen aus der Sicht eines Bundeslandes

Maximilian Gaßner, Dirk Göppfarth und Rüdiger Wittmann

Spielräume für eine Dezentralisierung im Rahmen des Systems

Gesundheitsfonds

Rolf Hoberg

Notwendigkeiten und Chancen einer dezentralen Gesundheitsversorgung

für eine landesweit kalkulierende Krankenkasse

Norbert Klusen

Möglichkeiten einer dezentralen Geschäftspolitik aus Sicht einer

bundesweit kalkulierenden Krankenkasse

Carl-Heinz Müller

Gemeinsame Bedarfsplanung für die regionale Versorgung aus Sicht

niedergelassener Ärzte

Joachim Bovelet

Flexibilisierung in der stationären und ambulanten Versorgung im Rahmen

eines öffentlichen Krankenhausverbundes.

Hagen Pfundner

Chancen und Grenzen der Individualisierten Medizin 
Wolf-Dieter Ludwig

Verfahren zur Frühbewertung des Nutzens neuer, patentgeschützter

Arzneimittel - Sicht der Arzneimittelkommission der deutschen

Ärzteschaft

Johann-Magnus von Stackelberg und Anja Olbrich

Erstattungsbedingungen nach dem Arzneimittelmarktneuordnungsgesetz (AMNOG)

Franz Waibel

Effekte der Frühbewertung patentgeschützter Arzneimittel auf die forschende pharmazeutische Industrie

Verzeichnis der Autoren 177 


\section{Begrüßung}

\section{Klaus Knabner}

Meine sehr geehrten Damen und Herren,

im Namen von Bayer HealthCare und Liam Condon, Geschäftsführer von Bayer HealthCare Deutschland, begrüße ich Sie ganz herzlich zu den 15. Bad Orber Gesprächen hier in Berlin. Leider musste Herr Condon aufgrund von dringlichen Terminen kurzfristig absagen, was er sehr bedauert. Genau vor einer Woche hat der Bundestag die beiden ersten Reformpakete der schwarz-gelben Koalition verabschiedet: am Donnerstag das Arzneimittelmarkt-Neuordnungsgesetz und am Freitag das GKV-Finanzierungsgesetz. Mit der Finanzreform, die auf dem Wettbewerbs-Stärkungsgesetz von 2007 aufsetzt, werden einkommensunabhängige Zusatzbeiträge als weiteres Finanzierungsinstrument eingeführt und mit einem neu konstruierten Solidarausgleich verbunden. Mit der Reform der Arzneimittelversorgung hat der Gesetzgeber die Reset-Taste für die Nutzenbewertung gedrückt. Von wenigen Ausnahmen abgesehen müssen sich nun alle neuen Wirkstoffe binnen eines halben Jahres einer Evaluierung ihres Zusatznutzens stellen, und dabei entscheidet sich, ob ein neues Arzneimittel sogleich unter Festbetrag kommt oder ob - in zentralen Verhandlungen mit dem GKVSpitzenverband - ein Erstattungshöchstbetrag vereinbart werden muss. Aus dieser Reform sind zwei zentrale Institutionen des Gesundheitswesens gestärkt hervorgegangen: Der Gemeinsame Bundesausschuss, dem die schnelle Nutzenbewertung obliegt, und der GKV-Spitzenverband, mit dem die Erstattungshöchstbeträge auszuhandeln sind. Bedenkt man, dass diese Institutionen Entscheidungen treffen, die auch für die private Krankenversicherung und die Beihilfe - und somit für alle Selbstzahler - Wirkung haben, so haben wir es im Ergebnis mit einer starken Zentralisierung und Monopolisierung zumindest für die Arzneimittelversorgung zu tun. Dass dies unter der Federführung eines liberalen Gesundheitsministers so entschieden worden ist, ist eine Überraschung.

Eher wäre aus meiner Sicht zu erwarten gewesen, dass der Ordnungsrahmen in der Gesundheitspolitik mehr Liberalisierung, mehr Dezentralität und mehr Wettbewerb schafft. Nun sind allerdings die beiden Reformen, die vor einer Woche beschlossen wurden, mit Sicherheit nicht die letzten in dieser Legislaturperiode. Angekündigt ist eine Reform der Versorgung, wahrscheinlich in Verbindung mit einem neuen Vergütungssystem für die Ärzte und einer neuartigen 
Bedarfsplanung - beides Elemente, mit denen auf absehbaren Ärztemangel reagiert werden soll. Ferner steht eine Reform der Pflegeversicherung auf der Agenda. Ebenso sollen der Gesundheitsfonds und insbesondere der morbiditätsorientierte Risikostrukturausgleich überprüft werden.

Meine sehr geehrten Damen und Herren,

angesichts der noch anstehenden Reformnotwendigkeiten haben wir mit Bedacht die diesjährigen Bad Orber Gespräche unter das Generalthema „Dezentralisierung und Flexibilisierung im Gesundheitswesen" gestellt. Ich freue mich deshalb ganz besonders, den Leiter der Grundsatzabteilung im Bundesgesundheitsministerium, Christian Weber, zu begrüßen, der über „Ansatzpunkte einer Dezentralisierung aus der Sicht des Bundesgesundheitsministeriums" referieren wird. Wir stellen fest, dass derzeit wesentliche Bestimmungsfaktoren für die Gesundheitsversorgung zentral nach einheitlichen Prinzipien festgelegt werden: Es ist der Gesundheitsfonds mit dem Morbi-RSA, der die Zuweisungen an die Krankenkassen zentral bestimmt. Das gleiche gilt für die Vergütung der Vertragsärzte, deren Honorare nach einem gesetzlich vorgegebenen, vertraglich vor allem von $\mathrm{KBV}$ und GKV-Spitzenverband im gemeinsamen Bewertungsausschuss beschlossenen Algorithmus an die Kassenärztlichen Vereinigungen und an die Ärzte verteilt werden. Neben der hohen Komplexität ist auch massive Kritik an den regionalen Verteilungseffekten insbesondere zu Lasten der reicheren Bundesländer Kritik geübt worden. Zu Diskussion mag auch stehen, ob die Vergütungen für die Krankenhäuser in ein komplett einheitliches System mit BasisFallwerten konvergieren, die dann keine regionalen Unterschiede mehr aufweisen. Es sind heiße Eisen, die der Gesetzgeber hier anzupacken hat.

Ich freue mich, Professor Günter Neubauer, dem Leiter vom Institut für Gesundheitsökonomik, zu begrüßen, der uns aus der Sicht des Gesundheitsökonomen Ziele und Optionen einer Dezentralisierung systematisieren wird. Ich hoffe, er wird uns einen roten Faden zeigen, der uns bei widerstreitenden Interessen hilft, zu den Gesetzen der Logik zurückzukehren - oder zumindest daran Orientierung zu finden.

Eine besonders spannende Region ist seit einiger Zeit Baden-Württemberg. Das neben Bayern wohlhabendste Flächenland ist zum einen betroffen vom morbiditätsorientierten Risikostrukturausgleich, weil Einnahmen der regionalen Kassen aufgrund der überdurchschnittlichen Einkommen im Südwesten aus die- 
sem Bundesland abfließen in die ärmeren Regionen. Ein ähnlicher Effekt ist seit 2009 in der vertragsärztlichen Versorgung zu beobachten. Aufgrund der neuen Honorarverteilungsregeln stagnieren die die Honorarsummen für die Vertragsärzte in Baden-Württemberg - ähnliche Effekte sind in Bayern und Nordrhein zu beobachten. Hingegen hat sich ein großer Teil der insgesamt beachtlichen Honorarzuwächse auf die Stadtstaaten Hamburg und Berlin sowie auf die neuen Bundesländer konzentriert. Ich freue mich deshalb, den Leiter der Gesundheitsabteilung des baden-württembergischen Sozialministeriums, Manfred Zach, zu begrüßen, der uns die Sichtweise eines von zentralen Steuerungsmechanismen besonders betroffenen Bundeslandes darstellen wird.

Der Gesundheitsfonds und mit ihm das Management des Morbi-RSA obliegt einer Abteilung des Bundesversicherungsamtes. Übrigens sehr effizient und ohne den einst behaupteten bürokratischen Aufwand erledigen diese Aufgabe gut 20 hoch qualifizierte Mitarbeiter des Bundesversicherungsamtes. Ich begrüße sehr herzlich dessen Präsidenten Dr. Maximilian Gaßner, der uns die Spielräume für eine Dezentralisierung im Rahmen des Gesundheitsfonds darstellen wird. Die spannende Frage wird hierbei sein, ob bestimmte regionale Komponenten bei der Mittelverteilung im Morbi-RSA berücksichtigt werden können - eine Forderung, die beispielsweise aus Bayern stammt. Sicherlich sollte dabei auch auf die Frage eingegangen werden, ob es im ersten Jahr, für das nun wohl die Endabrechnung vorliegt, zu den befürchteten Missbrauchsfällen gekommen ist, wie damit umgegangen worden ist und ob für die Zukunft Vereinfachungen im Morbi-RSA vorgesehen sind. Denkbar ist ja auch, dass es in Zukunft eine weitere Verfeinerung des Verteilungsmechanismus geben könnte, mit dem die bei einer Kasse versicherte Morbidität noch präziser abgebildet wird.

Nach der Kaffeepause haben dann die Praktiker von der Versorgungsfront das Wort. Die besonderen Herausforderungen für die Region Baden-Württemberg habe ich soeben schon dargelegt. Besonders interessant ist allerdings die Geschäftspolitik der AOK Baden-Württemberg, deren Vorstandsvorsitzenden Dr. Rolf Hoberg ich begrüße. Seine Kasse arbeitet systematisch und bisher mit beachtlichem Erfolg daran, in der ambulanten vertragsärztlichen Versorgung eine neuartige Vertragspartnerschaft mit dem Hausärzteverband und mit Medi zu begründen - und damit das bisherige Monopol der Kassenärztlichen Vereinigung zu brechen. Mit der Verbindung von $\S 73 \mathrm{~b}$ und 73c-Verträgen ist dies in BadenWürttemberg am weitesten gediehen. 
Unstrittig ist Herr Hoberg in Baden-Württemberg der Marktführer - auf der Bundesebene ist die Techniker Krankenkasse eine der stärksten. Angesichts der Fusionen in der jüngeren Vergangenheit wechselt die Marktführerschaft bisweilen - aber unstrittig ist, dass die TK zu den profiliertesten gehört. Deren Vorstandsvorsitzenden Professor Norbert Klusen begrüße ich sehr herzlich. Anders als die AOKen, die in der Region jeweils der direkte Vertragspartner von Ärzten und Krankenhäusern sind, lag das Vertragsgeschäft bei den Ersatzkassen auf der Landesebene stets bei der vdek-Landesvertretung. Das hat zu einem komplizierten Mechanismus in der regionalen Vertragspolitik der zentral organisierten Ersatzkassen über den vdek-Bundesverband in die jeweiligen regionalen Landesvertretungen geführt. Die Frage ist, ob die Ersatzkassen auch in der Region eine eigenständige Rolle anstreben und sich im Wettbewerb stärker profilieren werden.

Ich sagte eingangs, dass der Gesetzgeber sich voraussichtlich im kommenden Jahr mit einem Gesundheitsversorgungsgesetz befassen wird, und zwar vor dem Hintergrund künftig ernsthafter werdender Personalengpässe bei den beiden wichtigsten Berufsgruppen: den Ärzten und den Pflegekräften. Eines scheint absehbar: Ambulante und stationäre Medizin werden ihr sektorales Denken verlassen und in Zukunft wesentlich enger zusammenarbeiten und auch gemeinsame Kapazitäten, etwa in der Technik nutzen. Es scheint unter den unmittelbar Beteiligten Konsens zu sein, dass auch die personellen Kapazitäten gemeinsam geplant werden sollen. Ich begrüße ganz herzlich Dr. Carl-Heinz Müller, Vorstand der Kassenärztlichen Bundesvereinigung und Reinhard Nieper, ehemaliger Geschäftsführer der Alexianer $\mathrm{GmbH}$, einer der größten katholischen Träger von Gesundheitseinrichtungen in Deutschland.

Sie werden aus ihrer jeweiligen Sicht die Optionen und Notwendigkeit einer gemeinsamen Bedarfsplanung für die regionale Versorgung vorstellen.

Am morgigen Vormittag starten wir mit einem Blick in die Krankenhausversorgung. Am Beispiel Vivantes wird Herr Joachim Bovelet zeigen, wie die stationäre und ambulante Versorgung im Rahmen eines öffentlichen Krankenhausverbundes flexibilisiert werden kann. Welche Optionen der Dezentralisierung einer privaten Krankenhauskette offenstehen, wird Herr Dr. Gebhard von Cossel, Ärztlicher Direktor von den Sana Kliniken Berlin-Brandenburg GmbH darstellen. 
Den Abschluss unseres Symposions bildet ein Ausblick auf die Chancen und Herausforderungen der Arzneimittelversorgung unter den beschlossenen neuen gesetzlichen Rahmenbedingungen. Ich freue mich, dazu Dr. Hagen Pfundner, den Vorstand der Roche Pharma AG, zu begrüßen. Maßgeblich hat Roche das Konzept der personalisierten Medizin entwickelt - und damit Hoffnungen auf eine präzisere, effektivere Therapie vor allem für schwere und lebensbedrohliche Erkrankungen geweckt.

Immer wieder hat die Arzneimittelkommission der deutschen Ärzteschaft die unzureichende Studienlage insbesondere in der Onkologie beklagt. Ihren Vorsitzenden Professor Dr. Wolf Dieter Ludwig begrüße ich hier deshalb besonders herzlich. Wir erwarten von ihm Aufschluss, ob die Verfahren zur Frühbewertung patentgeschützter Arzneimittel ein Ansatzpunkt sein können, den Ärzten bei der Entscheidung über ihre Therapien eine bessere Evidenz an die Hand zu geben.

Schlussendlich haben zwei neue Vertragspartner das Wort: der GKVSpitzenverband und Unternehmen der forschenden pharmazeutischen Industrie. Johann Magnus von Stackelberg, der stellvertretende Vorstandsvorsitzende des GKV-Spitzenverbandes wird aus seiner Sicht die Erstattungsbedingungen von patentgeschützten Arzneimitteln erörtern - Dr. Franz Waibel, der bei der Bayer Schering AG den Market Access verantwortlich steuert, wird die Effekte der Frühbewertung patentgeschützter Arzneimittel auf die forschende Industrie erläutern.

Ganz besonders begrüße ich die beiden Moderatoren unseres Symposions, Gerhard Schulte und Professor Alfred Holzgrewe. Sie haben die verdienstvolle Aufgabe, Sie durch die Diskussion zu leiten - eine Debatte, die seit nunmehr 14 Jahren durch den sachlichen Austausch von Fakten und Argumenten geprägt ist. Die Tatsache, dass wir es geschafft haben, nunmehr $15 \mathrm{mal}$ in Kontinuität und unbeeinflusst von Firmenfusionen und vielfältigen anderen Veränderungen $\mathrm{zu}$ diesem Symposion zusammenzukommen, ist nicht zuletzt auch ein Erfolg der Teilnehmer selbst. Und natürlich auch seines Spiritus Rector, Professor Eberhard Wille, der mit einem großen Gespür für die relevanten Themen der Zukunft dieses Symposion geprägt hat und hoffentlich auch weiter prägen wird. Ich wünsche Ihnen neue Erkenntnisse und anregende Diskussionen.

Vielen Dank. 
Eberhard Wille and Klaus Knabner - 978-3-631-75550-1 Downloaded from PubFactory at 01/11/2019 03:39:43AM via free access 


\section{Ansatzpunkte einer Dezentralisierung in der Gesundheitspolitik}

\section{Christian Weber ${ }^{l}$}

\section{Zum Begriff Dezentralisierung}

Die Diskussion über eine Dezentralisierung im Gesundheitswesen ist vor allem als Antwort auf eine Gesundheitspolitik in der letzten Legislaturperiode zu verstehen, die stark von zentralisierenden Elementen gekennzeichnet war.

Zwar gibt es keine eindeutige Definition darüber, was genau mit Zentralisierung oder Dezentralisierung gemeint ist, gleichwohl enthält das aus der letzten Legislaturperiode stammende GKV-WSG (Gesetz zur Stärkung des Wettbewerbs in der gesetzlichen Krankenversicherung) zahlreiche Elemente, die unzweifelhaft eine stark zentralisierende Wirkung entfaltet haben.

Die Schaffung des Gesundheitsfonds mit einem kasseneinheitlichen Beitragssatz, der per Rechtsverordnung durch die Bundesregierung festzulegen ist, hat den bis zum In-krafttreten des GKV-WSG bestehenden Beitragswettbewerb allein auf die kassenindividuell unterschiedliche Höhe eines Zusatzbeitrags reduziert. Da dieser Zusatzbeitrag zudem durch ein enges Regelwerk definiert wurde, wonach er bis zu einer Höhe von $8 €$ pro Monat ohne Sozialausgleich berechnet wird, zugleich die jenseits von $8 €$ die für den Sozialausgleich erforderliche Finanzierung über den Zusatzbeitrag selber vorzunehmen war, wobei die maximale Höhe des Zusatzbeitrags auf 1 Prozent des beitragspflichtigen Einkommens begrenzt war, hat diese Regelung zwar zu beträchtlichen Verschiebungen der Mitgliederzahlen zwischen den Krankenkassen geführt, ein echter Beitragswettbewerb im Sinne eines funktionsfähigen Preiswettbewerbs als Ausdruck unterschiedlicher ökonomischer Leistungsfähigkeiten ist damit jedoch nicht verbunden gewesen.

Das GKV-WSG hat zudem durch die Umwandlung von Zusatzleistungen in Pflichtleistungen den Leistungskatalog der GKV über das ohnehin schon bestehende Ausmaß hinaus weiter vereinheitlicht.

Auch im Vertragsbereich hat es Zentralisierungen gegeben, die vor allem auch in einer Änderung der Verbändestruktur ihren Niederschlag fanden, indem

\footnotetext{
${ }^{1}$ Die Ausführung gibt die persönliche Auffassung des Autors wieder.
} 
der GKV-Spitzenverband an die Stelle von sieben kassenartenspezifischen Spitzenverbänden getreten ist.

Um dieses Gesetz herum hatte sich nicht zu Unrecht eine intensive Diskussion entwickelt, in der die eine Seite dem Wortlaut des Gesetzes zufolge das Ziel der Intensivierung des Wettbewerbs in den Vordergrund stellte, während die Gegenposition die Zentralisierung und die damit drohende schrittweise Entwicklung zu einer Einheitsversicherung in den Vordergrund stellte.

Diese divergierenden, im Widerspruch zueinander stehenden Auffassungen, sind vor allem unterschiedlichen Vorstellungen darüber geschuldet, was genau unter Wettbewerb im Gesundheitswesen zu verstehen ist. Die Befürworter des Wettbewerbsgedankens des GKV-WSG fordern insbesondere einen sogenannte Qualitätswettbewerb als Gegenentwurf zu einem zuweilen sogar als "ruinös" bezeichneten Beitragswettbewerbs unbedacht der Frage, ob eine "bessere Qualität" tatsächlich durch eine wettbewerbliche Vertragspolitik der Krankenkassen gestaltet werden kann und ob diese Qualität von den Versicherten als entscheidungsrelevantes Kassenwechselmerkmal überhaupt festgestellt und realisiert werden kann. Die Erfahrungen seit dem GKV-WSG sind unter Wettbewerbsaspekten wenig ermutigend:

- Weniger Qualitätsaspekte haben zu Wechselentscheidungen der Versicherten geführt, was im Übrigen schon alleine deshalb nicht zu erwarten war, weil die Wechselbereitschaft der Versicherten oft mit einem positiven Gesundheitszustand korreliert ist. Kranke, die viele Versicherungsleistungen in Anspruch nehmen, gehören eben gerade nicht zu den typischen Kassenwechslern.

- Maßgeblich für Wechselentscheidungen war vor allem die Frage, ob eine Krankenkasse einen Zusatzbeitrag berechnet, der jedoch nicht als Instrument eines funktionsfähigen Wettbewerbs konzipiert war.

- Unter Wettbewerbsaspekten ist auch die Möglichkeit des Angebots von Wahltarifen weit hinter den Erwartungen zurück geblieben. Nicht nur die absolute Zahl der Versicherten in den Wahltarifen ist gemessen an den Erwartungen gering geblieben, auch die Bedingungen für Wahltarife selber waren nicht wettbewerbsfördernd gestaltet. Die mit ihnen verbundene dreijährige Bindungsfrist der Versicherten an die jeweilige Krankenkasse ist von den meisten Krankenkassen insbesondere als strategisches Instrument der Abwanderungsverhinderung genutzt worden. 
- Als wichtiger Wettbewerbsparameter ist dann vor allem der versichertenspezifische Service der Krankenkassen geblieben. Dies ist jedoch weniger auf das GKV-WSG zurückzuführen, sondern letztlich auf die Einführung der Wahlfreiheit der Versicherten durch das Gesundheitsstrukturgesetz von 1992.

Die Auswirkungen dieses "Wettbewerbsprozesses" haben dann auch vor allem seinen Niederschlag in einer Beschleunigung von Fusionsprozessen geführt. Selbstverständlich gibt es auch in einem funktionierenden Wettbewerb Fusionsprozesse. Das Gesundheitswesen ist aber seit dem Inkrafttreten des GKV-WSG nicht nur durch eine ungewöhnliche Beschleunigung von Fusionsvorgängen gekennzeichnet gewesen, sondern diese Fusionen drohen auch über den Verbleib einer für den Wettbewerb notwendigen Anzahl von Konkurrenten weit hinauszugehen. Es kann geradezu als eine besonders gelungene Strategie der Zentralisierung bezeichnet werden, wenn Verhaltensanreize für die Akteure so geschaffen werden, dass sie diese Zentralisierung schrittweise über Fusionen auf freiwilliger Grundlage herbeiführen. Die so in Gang gesetzten Prozesse dann selbst noch als wettbewerbliches Handeln darzustellen, ist politisch und medial zwar geschickt, aber deshalb trotzdem keineswegs zutreffend. Einen Prozess zur Abschaffung des wettbewerblichen Handelns kann man auch dann nicht als Wettbewerb bezeichnen, wenn im Glauben weiter Teile der Öffentlichkeit die Zahl der Krankenkassen noch als zu groß empfunden wird. Zum Wettbewerb gehören Handlungsparameter. Reduziert man diese, in dem man beispielsweise den Preiswettbewerb einschränkt oder beseitigt, oder indem man den Leistungswettbewerb durch die weitere Vereinheitlichung des Leistungskatalogs reduziert, dann bleibt den Akteuren vor allem die Suche nach Synergieeffekten durch $\mathrm{Zu}$ sammenschlüsse.

Die Regierungskoalition dieser Legislaturperiode fand damit also zunächst eine Situation vor, in der zahlreiche mit dem WSG eingeleitete Schritte einen Zentralisierungsprozess eingeleitet haben, der mit den Grundregeln eines funktionsfähigen Wettbewerbsrechts kaum vereinbar ist. $\mathrm{Ob}$ am Ende dieses Prozesses tatsächlich eine Art "Einheitsversicherung" stehen würde oder ob, wie von vielen angenommen, eine begrenzte Anzahl von "Großkrankenkassen" übrig bleiben würde, ist eine eher theoretische Diskussion. 


\section{Dezentralisierung und Handlungsauftrag dieser Legislaturperiode}

Die Bundesregierung hat sich im Koalitionsvertrag festgelegt: "Der Weg in die Einheitskasse und in ein staatlich zentralistisches Gesundheitssystem sind der falsche Weg, um die zukünftigen Herausforderungen bürgernah zu bewältigen. Wettbewerb der Krankenversicherungen wirkt als ordnendes Prinzip mit dem Ziel der Vielfalt, der Effizienz und der Qualität der Versorgung". Dezentralisierung entspricht als Strategie dabei grundsätzlich dem Subsidiaritätsprinzip. Eine Entscheidungskompetenz ist von der unteren Einheit erst dann auf die übergeordnete Einheit zu verlagern, wenn die untere Einheit überfordert ist.

In den so geschaffenen Entscheidungsfreiheiten kann dann ein wirksamer Wettbewerbsprozess etabliert werden. Da Gesundheit bzw. Gesundheitsleistungen jedoch in weiten Teilen nur begrenzt marktfähige Güter sind, ist die Schaffung von Wettbewerbsstrukturen im Gesundheitswesen eine besonders anspruchsvolle Aufgabe. Dieses Spannungsverhältnis wird insbesondere in der gesundheitsökonomischen Literatur seit Jahrzehnten intensiv diskutiert. Versorgungssicherheit, gleicher Zugang zu Leistungen für alle sowie die Bezahlbarkeit von Leistungen und Versicherungsschutz für alle unter den Aspekten von Solidarität und Gerechtigkeit sind einige Anforderungen von großer Bedeutung. Es gilt den Wettbewerb als gestaltendes Steuerungsinstrument dort einzuführen, wo dies unter den Besonderheiten dem Gesundheitswesen und unter Einhaltung von weiteren Bedingungen machbar und sinnvoll ist. Eine so definierte Dezentralisierung dient vor allem dem Ziel der Schaffung von Wahl- und Gestaltungsfreiheiten.

Ziel der christlich-liberalen Regierungskoalition ist es deshalb, den Wettbewerb im Gesundheitswesen mit den geeigneten Instrumenten zu stärken. Für die Versicherten und Patienten bedeutet eine größere Wahlfreiheit am Ende auch eine bessere Behandlung. Mehr Wettbewerb im Gesundheitswesen steht für eine bessere medizinische Qualität, mehr Effizienz, geringere Kosten sowie weniger Bürokratie. Um dies zu erreichen, muss sich der Wettbewerbsgedanke in allen Bereichen des Gesundheitswesens stärker durchsetzen: zwischen den Krankenkassen, zwischen den Anbietern medizinischer Leistungen und im Verhältnis der Krankenkassen zu den Leistungserbringern - insbesondere zu Ärzten und Krankenhäusern. 
In einem gesunden und wettbewerblichen Krankenkassensystem konkurrieren die gesetzlichen Krankenkassen darum, wer seinen Versicherten das beste Angebot zum bestmöglichen Preis machen kann.

Mit dem aktuellen GKV-Finanzierungsgesetz werden insbesondere die bereits existierenden Zusatzbeiträge weiterentwickelt, um die Beitragsautonomie der Krankenkassen zu stärken. Der von den Versicherten zu leistende kassenindividuelle Zusatzbeitrag in Euro und Cent macht die Kassen vergleichbar. Die unterschiedliche Höhe der Zusatzbeiträge hat eine Preisfunktion, an der sich die Versicherten orientieren können. Ist der Zusatzbeitrag hoch und steht ihm kein zusätzlicher Nutzen - z. B. besserer Service oder bessere Versorgungsangebote gegenüber, werden sie zu einer Kasse wechseln, die einen geringeren Zusatzbeitrag erhebt. Die Kassen ihrerseits werden zukünftig ein größeres Interesse daran haben, kostengünstige Versorgungen anzubieten und dafür gute Verträge mit den Leistungserbringern zu schließen. Davon werden die Versicherten und das System insgesamt profitieren. Die Krankenkassen können den Zusatzbeitrag frei festlegen.

Die Schaffung von mehr Wettbewerb auf der Einnahmenseite kann jedoch nur ein erster Schritt sein. Ziel ist es, weitere strukturelle Reformen im System anzugehen, die zu mehr Wettbewerb, mehr Freiheit für den Einzelnen führen. Dies wird vor allem eine Aufgabe für das anstehende Versorgungsgesetz sein.

Das GKV-Finanzierungsgesetz setzt sich aus strukturellen Maßnahmen zur grundsätzlichen Neuordnung der Finanzierung (Weiterentwicklung der Zusatzbeiträge) und kurzfristigen Maßnahmen zur Konsolidierung der Finanzierungsgrundlagen der gesetzlichen Krankenversicherung zusammen:

1. Anhebung des allgemeinen Beitragssatzes auf 15,5 Prozent ( $\$ 241$ SGB V): Der mit Hilfe von Steuermitteln im Jahr 2009 abgesenkte Beitragssatz der Krankenkassen für die Beitragszahler (Arbeitgeber, RV-Träger, Bundesagentur für Arbeit und sonstige Stellen sowie Arbeitnehmer, Rentner und sonstige Mitglieder) wurde wieder auf 14,6 Prozent - wie vor der Senkung durch das Konjunkturpaket II - angehoben. Der nur vom Mitglied finanzierte Beitragssatzanteil von 0,9 Prozent bleibt erhalten.

2. Durchbrechung der automatischen Auswirkungen steigender Gesundheitsaus-gaben auf die Lohnkosten: Der allgemeine Beitragssatz wird für die $\mathrm{Zu}-$ kunft gesetzlich auf 15,5 Prozent fest geschrieben (§ 241 SGB V). Die bis- 
herigen Regelungsmechanismen (Rechtsverordnung; 95 Prozent-Klausel für Anhebung; 100 Prozent-Klausel für Senkung) entfallen. Über die Einnahmeentwicklung hinausgehende Ausgabensteigerungen werden künftig durch einkommensunabhängige Zusatzbeiträge der Mitglieder finanziert. Die Festschreibung des Arbeitgeberbeitrags auf 7,3 Prozent stabilisiert die Lohnkosten und verbessert die Wettbewerbsfähigkeit der deutschen Wirtschaft. Der bisherige Automatismus, dass Ausgabensteigerungen im Gesundheitsbereich zwangsläufig zu steigenden Lohnkosten führen, wird durch die Entkopplung durchbrochen. Damit werden langfristig Wachstum und Beschäftigung gefördert. Eine weitere Belastung des Faktors Arbeit mit der Folge des Abbaus sozialversicherungspflichtiger Beschäftigung wird vermieden. Durch das Umsteuern hin zu einkommensunabhängigen Zusatzbeiträgen wird die Finanzierungsgrundlage zugleich unabhängiger von konjunkturellen Schwankungen.

3. Weiterentwicklung der heutigen Zusatzbeiträge: Künftig ist nur noch die Erhebung von einkommensunabhängigen Zusatzbeiträgen möglich (§ 242 SGB V). Die Zusatzbeiträge selbst unterliegen künftig nicht mehr der Begrenzung auf 1 Prozent der beitragspflichtigen Einnahmen eines Mitglieds. Es gilt eine Überforderungsgrenze für den durchschnittlichen Zusatzbeitrag von 2 Prozent der beitragspflichtigen Einnahmen eines Mitgliedes.

4. Bei den Regelungen zum kassenindividuellen Zusatzbeitrag ist ein wirksamer Sanktionsmechanismus in Form eines Verspätungszuschlags für Mitglieder vorgesehen, die mit der Zahlung des kassenindividuellen Zusatzbeitrages für jeweils insgesamt sechs Monate säumig sind. Der Verspätungszuschlag ist in der Höhe auf die letzten drei fälligen Zusatzbeiträge begrenzt und muss mindestens 20 Euro betragen; das Nähere regelt hierzu die Krankenkasse in ihrer Satzung ( $\$ 242$ Abs. 6 SGB V). Bis zur Zahlung der ausstehenden Zusatzbeiträge und des Verspätungszuschlags besteht zudem kein Anspruch auf Sozialausgleich, es sei denn, dass eine wirksame Ratenzahlungsvereinbarung mit der Krankenkasse getroffen wurde und die Raten vereinbarungsgemäß gezahlt werden.

5. Der Schätzerkreis schätzt künftig im Herbst des jeweiligen Vorjahres in Form einer Gegenüberstellung die voraussichtlichen Ausgaben der Krankenkassen des kommenden Jahres und die voraussichtlichen Einnahmen des Gesundheitsfonds (einkommensabhängige Beiträge und sonstige Einnahmen wie Bundesmittel), die zur Deckung dieser Ausgaben zur Verfügung stehen sowie die voraussichtlichen Versicherten- und Mitgliederzahlen der Kran- 
kenkassen insgesamt. Auf Basis dieser Schätzungen legt das Bundesministerium für Gesundheit im Einvernehmen mit dem Bundesministerium der Finanzen den durchschnittlichen Zusatzbeitrag fest und gibt ihn bekannt (§ 242a SGB V). Der durchschnittliche Zusatzbeitrag ist für die Durchführung des Sozialausgleichs erforderlich.

6. Für bestimmte Mitgliedsgruppen wird dann kein Zusatzbeitrag erhoben, wenn sie (vorübergehend) über keine beitragspflichtigen Einnahmen verfügen. Dazu zählen Empfängerinnen und Empfänger von bestimmten Entgeltersatzleistungen wie Krankengeld, Verletztengeld, Versorgungskrankengeld oder Übergangsgeld sowie Mutterschaftsgeld oder Elterngeld. Ebenso von der Erhebung von Zusatzbeiträgen ausgenommen sind Teilnehmerinnen und Teilnehmer an Leistungen zur Teilhabe am Arbeitsleben, behinderte Menschen, die in anerkannten Werkstätten oder in Blindenwerkstätten tätig sind oder die in Anstalten, Heimen oder gleichartigen Einrichtungen in gewisser Regelmäßigkeit eine Leistung erbringen, Wehr- und Zivildienstleistende, Versicherte, die ein freiwilliges soziales oder ökologisches Jahr leisten, sowie Auszubildende mit einem Entgelt bis zu 325 Euro monatlich oder in einer außerbetrieblichen Einrichtung ( $\$ 242$ Abs. 5 SGB V). Verfügen diese Mitglieder über weitere beitragspflichtige Einnahmen, ist der Sozialausgleich im Regelfall lediglich auf der Bemessungsgrundlage dieser Einnahmen durchzuführen.

7. Für Mitglieder, die Arbeitslosengeld II oder Sozialgeld beziehen, wird der Zusatzbeitrag zwar erhoben, sie zahlen ihn aber künftig nicht selbst, sondern für diese Mitglieder wird der kassenindividuelle, höchstens jedoch der durchschnittliche Zusatzbeitrag ( $\$ 242$ Abs. 4 SGB V) aus den Mitteln der Liquiditätsreserve aufgebracht ( $\$ 251$ Abs. 6 SGB V). Ab 2015 wird die Zahlung dieses Zusatzbeitrages aus der Liquiditätsreserve beim Bundeszuschuss für den Sozialausgleich vollumfänglich berücksichtigt. Der Bund stellt für die Jahre 2011 bis 2014 zwei Mrd. Euro für den Sozialausgleich zur Verfügung. Damit ist die Finanzierung gewährleistet. Der zu zahlende $\mathrm{Zu}$ satzbeitrag wird im Verrechnungsverfahren aus der Liquiditätsreserve des Gesundheitsfonds an die jeweiligen Krankenkassen gezahlt. Ist der kassenindividuelle Zusatzbeitrag höher als der durchschnittliche Zusatzbeitrag, kann die Krankenkasse in ihrer Satzung vorsehen, dass diese Differenz vom Mitglied zu zahlen ist. Das gleiche Verfahren gilt auch für so genannte "Aufstocker". 
8. Für Bezieherinnen und Bezieher von Arbeitslosengeld I oder Kurzarbeitergeld gelten die allgemeinen Regeln, $d$. $h$. sie zahlen den kassenindividuellen Zusatzbeitrag selbst und erhalten bei Überforderung einen Sozialausgleich durch die Bundesagentur für Arbeit bzw. durch den Arbeitgeber.

Der Sozialausgleich ( $§ 242 b$ SGB V) wird i. d. R. durch den Arbeitgeber bzw. den Rentenversicherungsträger durchgeführt. Der einkommensabhängige Beitragssatzanteil des Mitglieds wird zu diesem Zweck um den Betrag gesenkt, um den das Mitglied durch Entrichtung des durchschnittlichen Zusatzbeitrags überfordert wäre. Für die Arbeitnehmerin und den Arbeitnehmer bzw. die Rentnerin und den Rentner bedeutet die Durchführung des Sozialausgleichs damit, dass sie von ihrem Arbeitgeber bzw. dem Rentenversicherungsträger entsprechend mehr ausbezahlt bekommen:

1. Der Sozialausgleich wird immer dann automatisch durchgeführt, wenn ein Mitglied nur aus einer Quelle beitragspflichtige Einnahmen bezieht, also z. B. bei einer Arbeitnehmerin bzw. einem Arbeitnehmer, die bzw. der über keine weiteren beitragspflichtigen Einkünfte verfügt (z. B. Einkommen aus anderen sozialversicherungspflichtigen Beschäftigungsverhältnissen, Renten etc., $\S 242$ b Absatz 2 SGB V).

2. Bezieht ein Mitglied Einkünfte aus mehreren beitragspflichtigen Einnahmen, so prüft die Krankenkasse die Anspruchsberechtigung auf einen Sozialausgleich und informiert die jeweiligen beitragsabführenden Stellen über das Prüfergebnis und das anzuwendende Verfahren zur Beitragsbemessung (§ 242b Abs. 3 SGB V neu). Der Sozialausgleich wird dann grundsätzlich von der beitragsabführenden Stelle ausgeführt, bei der das Mitglied sein Haupteinkommen bezieht. Ausnahmsweise führt bei Bezieherinnen und Beziehern einer gesetzlichen Rente immer der Rentenversicherungsträger den Sozialausgleich durch, soweit die Höhe der Rentenzahlung 260 Euro übersteigt.

3. Für freiwillig Versicherte, wie Selbständige, freiwillig versicherte Arbeitnehmerinnen und Arbeitnehmer, freiwillig versicherte Rentnerinnen und Rentner und Studentinnen und Studenten, die ihren Krankenkassenbeitrag selbst zahlen, wird der Sozialausgleich direkt von der Krankenkasse durchgeführt. Hierfür liegen der Krankenkasse bereits heute alle erforderlichen Informationen vor.

4. Für Bezieher von Arbeitslosengeld I gibt es keinen Beitragssatzanteil des Mitglieds, der entsprechend verringert werden kann. Daher erhalten sie von 
der Bundesagentur für Arbeit eine zusätzliche Auszahlung in Höhe der Überforderung. Die Bemessungsgrundlage für den Sozialausgleich liegt bei ihnen bei 67 Prozent des der Leistung zugrunde liegenden Arbeitsentgelts.

5. Für das Jahr 2011 wurde mit Bekanntmachung im Bundesanzeiger ein durchschnittlicher Zusatzbeitrag von 0 Euro festgelegt, da in diesem Jahr die Zuweisungen aus dem Gesundheitsfonds GKV-weit ausreichen werden, um die voraussichtlichen Ausgaben zu decken. Damit ist im Jahr 2011 kein Sozialausgleich notwendig.

Zugleich wurde das GKV-Finanzierungsgesetz von einem Bündel an Maßnahmen auf der Ausgabenseite der GKV begleitet, die vor allem zum Ziel hatte, das für $2011 \mathrm{zu}$ erwartende Finanzierungsdefizit auszugleichen.

Diese Maßnahmen sollten im Folgenden nicht im Einzelnen aufgeführt werden. Insgesamt ist damit das Ziel verfolgt worden, die GKV auf eine stabile Finanzierungsgrundlage für die Zukunft zu stellen. Soweit zukünftige Kostenentwicklungen über den Einnahmerahmen der aus der Beitragssatzfinanzierung gewonnenen wird, hinausgehen, wird dies ausschließlich seinen Niederschlag in der Höhe der Zusatzbeiträge finden. Da die Kassen über die Höhe der Zusatzbeitrags in einem direkten Wettbewerb stehen, werden sie in Zukunft ein einen direkten Preis- und Leistungswettbewerb eintreten.

\section{Weitere Schritte der Dezentralisierung}

Das GKV-Finanzierungsgesetz ist Teil einer Dezentralisierungsstrategie, die in vielen Maßnahmen im Laufe der Gesetzgebung diese Legislaturperiode ihre Fortsetzung finden wird. Den gewollten Wahlrechten der Versicherten müssen die geeigneten Handlungs- und Gestaltungsparameter auf der Leistungsseite der Krankenkassen gegen-übersehen. Hier gibt es eine ganze Reihe von Aspekten, die zum Teil im GKV-Finanzierungsgesetz schon aufgegriffen wurden, über deren Weiterentwicklung aber noch innerhalb dieser Legislaturperiode zu diskutieren und zu entscheiden sein wird. Einige Beispiele seien im Folgenden herausgegriffen.

Im GKV-Finanzierungsgesetz sind die Möglichkeiten zur Inanspruchnahme der Kostenerstattung verbessert worden. Es entbehrt in der politischen Diskussion nicht eines gewissen Reizes, die Kontroverse zwischen dem Sachleistungsprinzip und der Kostenerstattung aufzurufen. Unbestreitbar waren die vor dem 
GKV-Finanzierungsgesetz bestehenden Regelungen eines Wahlrechts für die Inanspruchnahme der Kostenerstattung anstelle der Sachleistung durch GKVVersicherte vor allem so gestaltet, dass das Wahlrecht zwar formal bestehen sollte, materiell aber für den Versicherten mit hohen Hürden verbunden sein sollte. Ziel der früheren Gesetzgebung war es offensichtlich gerade nicht, dem Versicherten ein faires Wahlrecht zu geben. Die Sachleistung wird von seinen Befürwortern als eine der zentralen Qualitätsmerkmale der GKV angesehen. Jeder kleine - und sei es nur als Wahlrecht ausgestalteter Einstieg in die Kostenerstattung - wird von den Befürwortern geradezu als Angriff auf die Fundamentalprinzipien der GKV angesehen. Die Diskussion hat damit einen hohen Ideologiegehalt, der für Außenstehende nur schwer nachvollziehbar ist.

Das Ziel der Koalition ist auch hier keine "entweder- oder -Entscheidung" zwischen Sachleistung und Kostenerstattungswesen, sondern dem Versicherten sollten Wahlfreiheiten gegeben werden. Die Wahl für das eine oder andere System mag mit Vor- oder Nachteilen verbunden sein, diese zu bewerten und danach zu entscheiden, sollte aber Aufgabe des Versicherten sein. Zu einer Strategie der Dezentralisierung gehört es, dass der Versicherte seine Wahl in eigener Verantwortung treffen kann und dass er diese Entscheidung auch ggf. wieder revidieren kann.

Im GKV-Finanzierungsgesetz sind deshalb die Regelungen für die Inanspruchnahme der Kostenerstattung durch die Versicherten liberalisiert worden. Ein strategischer Systemwechsel zu einem generellen Übergang auf die Kostenerstattung für alle und die Abschaffung der Sachleistung verbindet sich damit ausdrücklich nicht. Und es handelt sich auch nicht, wie insbesondere von Kreisen der Opposition behauptet wurde, um ein System der Vorkasse. Wer sich für die Kostenerstattung entscheidet, bekommt vom Arzt nach der Behandlung eine Rechnung nach der Gebührenordnung für ärztliche Leistungen (GÖ̈̈). Kein Arzt therapiert erst nach Vorauszahlung und dies wäre nach der GOÄ auch nicht statthaft.

Insbesondere im Bereich der ärztlichen Leistung kann die Kostenerstattung für den Versicherten deshalb mit zusätzlichen Kosten verbunden sein, weil eine Erstattung durch die Krankenkasse bis zur Höhe der bei Inanspruchnahme der Sachleistung für die Kasse verbundenen Kosten in der Regel nicht die Höhe der tatsächlichen Arztrechnung erreicht. Der Versicherte geht also einen finanziellen Nachteil ein, der im Einzelfall durchaus erheblich sein kann. Diesen hat er dann 
insgesamt mit den der Kostenerstattung verbundenen Vorteilen gegenüberzustellen. In aller Regel macht dies entweder die Entscheidung für einen kassenspezifischen Wahltarif erforderlich, der aber zur Zeit nur von ganz wenigen Krankenkassen angeboten wird, oder aber die Entscheidung für eine private Zusatzversicherung.

Es wäre eine spannende Diskussion, welche weiteren Möglichkeiten denkbar wären, um für den Versicherten das Kostendifferenzrisiko bei der Inanspruchnahme der Kostenerstattung tragbar zu machen. Hier lassen sich auch vertragliche Möglichkeiten zwischen Krankenkassen und Ärzteschaft diskutieren. Ob eine solche Diskussion allerdings vorbehaltlos in eine stark ideologisch aufgeladene Atmosphäre passt, bleibt abzuwarten.

Eine weitere Regelung im Bereich der Kostenerstattung ist zu erwähnen. Patienten, die sich in der Apotheke anstelle eines Arzneimittels, für das ihre Krankenkasse einen Rabattvertrag abgeschlossen hat, für ein anderes - gleichartiges - Arzneimittel entscheiden, sollten dies können, wenn sie bereit sind, die damit verbundenen Mehrkosten zu bezahlen. Organisiert wird diese Möglichkeit über das Instrument der Kostenerstattung.

Auch hier sollte es nicht darum gehen, die Diskussion über die Gleichartigkeit des Arzneimittels zu führen. Ein gleichartiges, aber teures Arzneimittel zu wollen, mag pharmakologisch wenig begründbar sein, aber die Entscheidungsfreiheit des Versicherten zu respektieren, sollte in einer liberalen Gesellschaft eigentlich eine Selbstverständlichkeit sein. Es ist kein zu rechtfertigender Tatbestand, wenn man Versicherten Wahlfreiheit einräumt. Es ist vielmehr ein rechtfertigender Tatbestand, wenn in Inanspruchnahme von Wahlfreiheiten beschneidet.

Ein weiteres wichtiges Instrument der Dezentralisierung und der Schaffung von Wahlfreiheiten der Versicherten können die Wahltarife sein. Die Erfahrungen seit dem WSG sind jedoch keineswegs uneingeschränkt positiv ausgefallen. Zwei Sachverhalte sind insbesondere festzustellen gewesen:

- Die bisherige Mindestbindungsfrist von 3 Jahren, die vor allen Dingen gedacht war, um eine solide Kalkulationsgrundlage zu schaffen, hat sich oft mehr als Wettbewerbsverhinderungsinstrument erwiesen, indem die Wahltarife vor allem "abwanderungswilligen" Versicherten angeboten werden. 
- Das Verbot von Quersubventionierungen aus allgemeinen Haushaltsmitteln der jeweiligen Kassen ist mit den bestehenden Regelungen nur schwer einzuhalten gewesen. Dies hat die Wahltarife dem Vorwurf der Solidaritätsgefährdung ausgesetzt.

Die Regierungskoalition hat deshalb an beiden Punkten angesetzt und die Mindestbindungsfrist für die Wahltarife (Ausnahmen: Krankengeld, Selbstbehalt) auf ein Jahr reduziert. Außerdem gilt in Zukunft ein Sonderkündigungsrecht (Ausnahme: Krankengeld), wenn die Kasse die Höhe ihres Zusatzbeitrags verändert. Zur Vermeidung von Quersubventionierungen müssen die Berechnungsgrundlagen künftig auf versicherungsmathematischen Grundlagen erstellt werden.

Damit ist das Instrumentarium der Wahltarife deutlich verbessert worden. Ohnehin wird jede Krankenkasse für sich die Entscheidung treffen müssen, ob die individuelle Zuwahl von Leistungen, die eigentlich nur zu bei einer risikogerechten Beitragskalkulation funktionieren kann, nicht besser durch private $\mathrm{Zu}$ satzversicherungen aufgestuft werden kann.

Das Sozialgesetzbuch eröffnet hier ein breites Spektrum an Kooperationsmöglichkeiten, die bisher deutlich häufiger als das Angebot von Wahltarifen genutzt worden sind.

Insbesondere im Zuge der in diesem Jahr anstehenden weiteren gesetzgeberischen Maßnahmen wird es auch um weitere Maßnahmen einer Dezentralisierung gehen.

Mit einiger Spannung kann man hierzu im Rahmen der geplanten Versorgungsgesetztes die Diskussion zur Bedarfsplanung verfolgen. Offensichtlich haben Bund und Länder hier ein unterschiedliches Verständnis von Dezentralisierung. Ohne die zum Teil stark divergierenden Positionen hier wiederzugeben, scheint einer Mehrheit der Länder der eigene gesundheitspolitische Gestaltungseinfluss deutlicher wichtiger zu sein als eine Dezentralisierung, die den Versicherten, den Leistungsanbietern und den Kassen mehr Wahlfreiheiten zubilligt.

Vereinfacht gesprochen ist der Ruf nach Dezentralisierung zugunsten einer Stärkung der Länderkompetenzen deutlich vernehmbarer als im Bereich des selektiven Kontrahierens den Vertragspartnern auch nur in kleinen Schritten entgegenzukommen. 
Das Nein zum selektiven Kontrahieren im Krankenhausbereich ist bestenfalls mit der Investitionskostenfinanzierung und dem Sicherstellungsauftrag der Länder begründbar. Die tatsächliche Höhe der übernommenen Investitionskostenfinanzierung sollte eigentlich eine flexiblere Einstellung bei der Forderung nach mehr selektiven Vertragsrechten rechtfertigen können. Anstelle dessen aber ein Mehr an Planungskompetenzen zu fordern, ist jedenfalls mit einer Politik der Dezentralisierung nicht begründbar. Die Diskussion wird also noch spannend werden.

Im Zusammenhang mit dem Versorgungsgesetz wird es auch um weitere Maßnahmen gehen, die es den Kassen durch Bemühungen von eigener Wirtschaftlichkeit ermöglichen, direkten Einfluss auf die Höhe der von ihnen zu berechnenden Zusatzbeiträge zu nehmen. Es wird darum gehen, den wettbewerbsrechtlichen Gestaltungsspielraum der Kassen möglichst weiter auszubauen. 
Eberhard Wille and Klaus Knabner - 978-3-631-75550-1 Downloaded from PubFactory at 01/11/2019 03:39:43AM via free access 


\title{
Ziele und Optionen einer Dezentralisierung
}

\author{
Günter Neubauer
}

\section{Ausgangslage: Zentralisierung und Dezentralisierung als normative Zielsetzungen}

Die derzeitige Diskussion über die Ziele einer Dezentralisierung bzw. Zentralisierung wird von der politisch gesetzten normativen Ausgangslage bestimmt. Auf der einen Seite wird eine gleiche Versorgung für alle Bürger im Land gewollt. Dabei liegt die Betonung auf „gleiche Versorgung“ und nicht nur gleichmäßige Versorgung. Hier ist bereits schon früher die Diskussion in Deutschland ganz allgemein über die gleiche bzw. gleichmäßige Verteilung der Infrastruktur geführt worden. Soll das Land jedem Bürger die gleiche Infrastruktur anbieten müssen, unabhängig vom Wohnort und unabhängig von seinen beruflichen Tätigkeiten? Daneben steht das normativ gesetzte Ziel, ein Land nach Regionen und Wohlstand, aber auch nach den Präferenzen der Bevölkerung differenziert zu versorgen.

Letztere Zielsetzung wird heute mehr und mehr bestritten. Einerseits wird eine regional differenzierte Gesundheitsversorgung als besonders benachteiligend für die ärmeren Regionen, und andererseits wird eine Gesundheitsversorgung nach Wohlstand als sozial diskriminierend eingestuft. Selbst eine Gesundheitsversorgung, die den unterschiedlichen Präferenzen der Bevölkerung folgt, wird noch für sozial bedenklich gehalten.

Ein Grund dafür, dass eine differenzierte Versorgung mehr und mehr in Zweifel gezogen wird, liegt auch daran, dass durch die Mobilität der Bevölkerung paradoxerweise eine gleiche Versorgung stärker eingefordert wird, obwohl ja eine mobile Bevölkerung auch zu dem Ort der besseren Versorgung wandern könnte. Gerade letzteres wollen aber vor allem Kommunal- und Landespolitiker verhindern. Dass dahinter auch Sorgen über die Wahlchancen einzelner Abgeordneter, aber auch ganzer Parteien stehen, kann man nur vermuten.

Fassen wir zusammen, es gibt zwei divergierende Zielsetzungen, die sich auch in den jeweiligen Gesundheitsprogrammen entsprechend niederschlagen: 
1) Gleiche Versorgung für alle Bürger eines Staates

2) Differenzierte Versorgung nach Regionen, Wohlstand und Präferenzen der Bürger

Je nachdem welche Zielsetzung angestrebt wird, ergeben sich, daraus quasi logisch ableitbar, die jeweiligen geeigneten Instrumente.

Betrachten wir die Gesundheitsreform der großen Koalition, so lässt sich dort eindeutig ein Trend zu mehr zentralisierter Steuerung, und damit zu einer gleichen Versorgung in Deutschland ableiten. So ist die Einführung des Gesundheitsfonds und der Einheitsbeiträge für Krankenkassen ein Schritt zur Zentralisierung, hinter dem eine gleiche Versorgung im Raum als Ziel steht. Auch die angestrebten, bundesweiten Einheitspreise auf der Leistungsseite zählen hierzu. $\mathrm{Zu}$ nennen sind der angestrebte, bundesweite Basisfallwert für die Krankenhausvergütung, der einheitliche Punktwert für die vertragsärztliche Versorgung, aber auch - nicht zu vergessen - die einheitlichen Abgabepreise für Medikamente aus Apotheken.

Ob die schwarz-gelbe Regierung eine stärkere Dezentralisierung der Gesundheitsversorgung anstrebt, und damit eine Reform der Vorgängerregierung einleitet, kann in Ansätzen erkannt werden. Doch soll dies für unsere weitere Diskussion nur von peripherem Interesse sein. Wir wollen im Folgenden prüfen, welche Optionen sich bei einer gewünschten Dezentralisierung anbieten.

\section{Handlungsoptionen für Zentralisierung und Dezentralisierung}

Beim weiteren Vorgehen differenzieren wir die GKV-Gesundheitsversorgung gemäß der Abb. 1 in drei Subsysteme bzw. Teilmärkte. Wenn wir hier von Märkten sprechen, so steht hier nicht der ordnungspolitische, sondern der technisch-organisatorische Aspekt im Mittelpunkt. Markt ist in diesem Sinne der Ort, an dem Angebot und Nachfrage zusammentreffen, unabhängig von der Art der Koordination. Zu beachten ist, dass wir dem Versicherten zwei Rollen zuordnen: Im Finanzierungsmarkt die des Beitragszahlers, im Leistungsmarkt die des Patienten und Leistungsempfängers. Leistungserbringern (LE), nämlich Ärzte, Krankenhäuser, Apotheken sowie Heil- und Hilfsmittelerbringer, stehen die Krankenkassen (KK) gegenüber. 
Abbildung 1: Teilmärkte der GKV-Gesundheitsversorgung

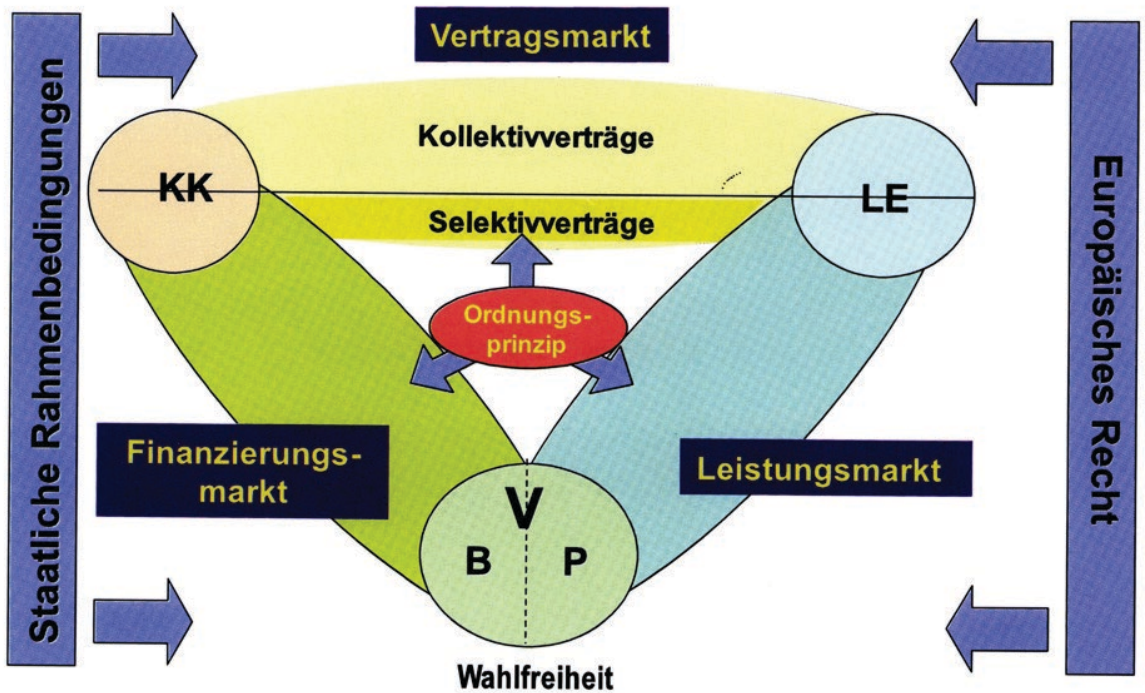

\section{Soziale Marktwirtschaft}

Quelle: G. Neubauer, in BKK 9/2002

\subsection{Im Finanzierungsmarkt}

Strebt man eine Zentralisierung auf der Finanzierungsseite des deutschen Gesundheitssystems an, so ist das Mittel der Wahl ein Einheitsbeitrag, der deutschlandweit einheitlich vom Staat festgelegt wird. Ein Schritt, der von der großen Koalition im Rahmen des GKV-WSG gemacht wurde. Die einheitliche Belastung der Beitragspflichtigen nach Zahlungsfähigkeit heißt aber nicht, dass auch eine einheitliche Versorgung erreicht wird.

Würde man hingegen Dezentralisierung auf der Finanzierungsseite wollen, so sind regional differenzierte Beitragssätze, je nach Zahlungswilligkeit und Versorgungsrealität, das Mittel der Wahl. Es müsste den Bürgern ein Stück weit überlassen bleiben, wie viel sie für Gesundheit mehr ausgeben wollen als andere. Eine bestimmte Grundversorgung kann dabei, insbesondere in der Notfallversorgung, gleichwohl überall einheitlich vorgehalten werden. Dabei ist die Höhe der Ausgaben nicht gleichbedeutend mit einer besseren Versorgung. Sie 
drückt in erster Linie aus, dass Bürger in einer Region über eine höhere Zahlungsfähigkeit und Zahlungswilligkeit verfügen, als Bürger in einer anderen Region. Dass eine solche Versorgung vor allen Dingen von den wohlhabenderen Bundesländern favorisiert wird, liegt auf der Hand. Schließlich könnten sie, bei gleicher relativer Belastung, ihrer Bevölkerung eine höhere Gesundheitsversorgung anbieten.

Um im Finanzierungsmarkt eine Dezentralisierung anzustreben, wären zunächst für Deutschland vor allen Dingen die einheitlichen Beitragssätze der Krankenkassen abzuschaffen. Das bedingt aber auch gleichzeitig, dass der Gesundheitsfonds neu konstruiert wird. Schließlich werden heute die Krankenkassen durch einheitliche Zuweisungen aus dem Gesundheitsfonds finanziert, und nicht mehr durch die Beitragssätze ihrer Versicherten. Es würde also bedingen, dass einerseits die Zuweisungen des Fonds eine dezentrale Komponente erhalten, die wir mit Regionalisierung bezeichnen wollen. Eine solche Regionalisierung könnte nach der regionalen, oder auch lokalen Kostenstruktur und Versorgungsdichte abgestuft werden. Es bleibt aber trotzdem unabdingbar, dass den Krankenkassen eine eigene dezentrale Finanzierungsautonomie zugesprochen wird, was eine Rückführung des Gesundheitsfonds bedingt. Dass die derzeitige Koalition (2010) dazu nicht die Kraft hat, zeigt das jüngst beschlossene GKVFinanzierungsgesetz.

Einen Schritt weiter auf der Finanzierungsseite sollte man insofern gehen, als man auch den bundesweiten Krankenkassen einen regionalisierten Beitragssatz erlaubt. Ein solcher regionalisierter Beitragssatz würde vor`allen Dingen den großen Ersatzkassen eine Differenzierung ihrer Beitragssätze nahe legen und sie dadurch im Wettbewerb stärker machen. Heute müssen wir vermuten, dass durch die einheitlichen Beitragssätze die wohlhabenden Regionen weniger Beitrag aufbringen, als ihrer hohen Versorgung adäquat wäre, und umgedreht weniger versorgte Gebiete einen relativ zu hohen Beitragssatz entrichten müssen.

\subsection{Im Vertragsmarkt}

Im Vertragsmarkt scheinen bei einem politischen Bestreben nach einer gleichen Versorgung gleiche Preise, d. h. staatlich festgesetzte Einheitspreise, geboten zu sein. Doch wissen wir, sowohl aus der Theorie wie aus der Praxis, dass mit administrativ festgesetzten Einheitspreisen keine einheitliche Versorgung zu errei- 
chen ist. Dennoch ist in der Realität immer wieder zu beobachten, dass die staatliche Preisfestsetzung eine hohe Priorität hat.

Will man jedoch Dezentralisierung anstreben, so sind flexible Verhandlungspreise, je nach lokaler oder regionaler Versorgungssituation, geboten. Durch flexible Verhandlungspreise werden Leistungsmenge und Leistungsqualität vor Ort gesteuert, was quasi automatisch zu unterschiedlichen Preisen und Vergütungen, je nach Angebot und Nachfragesituation, führen wird. Dass damit auch eine höhere und niedrigere Versorgung verbunden sein kann, liegt auf der Hand. Mittelbis langfristig führen flexible Preise zu einer Angleichung der Versorgung. Schließlich wandern die Anbieter zum Ort der höheren Vergütung, während sich umgedreht die Nachfrager zum Ort der niedrigeren Preise hin bewegen. Durch diese Wanderungsbewegungen, die zwar im Gesundheitssektor nur langsam ablaufen, kommt es quasi über den Vergütungsmechanismus zu einer Angleichung der Versorgung. Freilich wird es nie zu einer völligen Angleichung kommen, da in dynamischen Versorgungsbereichen sich stetig Veränderungen ergeben, und es schließlich auch immer verschiedene Präferenzen der Bevölkerung über ihren Lebens- und Wohnort geben wird.

Will man die Dezentralisierung im Vertragsmarkt unterstützen, so sind vor allem die Möglichkeiten für lokale und regionale Verträge mit Arztnetzen und Krankenhäusern zu unterstützen. Schließlich sind Gesundheitsleistungen immer Loco-Leistungen, also Leistungen, die örtlich gebunden sind. Eine solche regionale bzw. lokale Vertragsstruktur würde auch entsprechende flexible Vergütungssysteme zulassen müssen. Damit ergäbe sich automatisch eine dezentrale preisliche Steuerung, die, wie schon oben kurz angesprochen, auf lange Sicht die Versorgung stärker angleichen würde, als dies zentral festgelegte Einheitspreise zu leisten vermögen.

\subsection{Im Leistungsmarkt}

Im Leistungsmarkt können sich flexible, dezentrale Vergütungen und Preise nur entwickeln, wenn Ärzte wie Patienten auch ein gewisses Maß an Flexibilität und Mobilität entwickeln. Für Ärzte bedeutet das, dass regionale Niederlassungsfreiheit und keine einengende Bedarfsplanung bestehen muss. Von dem Patienten muss ein Mindestmaß an Preiselastizität gefordert werden. Über eine direkte prozentuale Kostenbeteiligung lässt sich erreichen, dass Patienten preisgünstigere Leistungserbringer bevorzugen, ohne auf die Leistungen selbst zu verzichten. 
Ohne eine direkte Einbindung der Patienten und Versicherten in die Preisbildung läuft das System Gefahr, dass sich Preise bilden, die nicht die wahren Präferenzen der Bevölkerung abbilden. Ein gutes Beispiel hierfür sind die vielen kleinen Krankenhäuser, deren Erhaltung von der jeweiligen Bevölkerung vehement gefordert wird. Käme es hier zu einer dezentralen Preisbildung der Krankenkassen mit den einzelnen Krankenhäusern, so müsste die Bevölkerung sehr schnell entscheiden, ob sie bei kleinen, unwirtschaftlichen Krankenhäusern bereit ist, sich an den höheren Preisen zu beteiligen, oder lieber entferntere, aber qualitativ bessere Krankenhäuser aufsucht.

\section{Theoretische Überlegungen zu zentralen Einheitspreisen und dezentra- len Wettbewerbspreisen}

Nachfolgend wollen wir zunächst kurz unsere Ausgangsthese auf preistheoretischer Grundlage erklären und dann die Konsequenzen ableiten, die sich für eine dezentrale Steuerung ergeben.

\subsection{Das Ausgangstheorem}

Hier greifen wir auf die traditionelle Preistheorie zurück, die besagt, dass sich die Preise in einem freien Wettbewerbsmarkt aus den Angebots- und Nachfragesituationen ergeben. In der nachfolgenden Abbildung haben wir drei AngebotsNachfrage-Relationen abgebildet: Einmal das relative Hochpreisland Hamburg mit einem überdurchschnittlich hohen Preis, bzw. hoher Vergütung für Krankenhausleistungen; darunter das Land Schleswig-Holstein, das bundesweit mit die niedrigsten Vergütungssätze für Krankenhäuser aufweist. Wir gehen davon aus, dass dies eine Konsequenz der Versorgungssituation ist, die sich wiederum aus den Angebots- und Nachfrageverhältnissen in den Bundesländern ableiten lassen. Und schließlich bilden wir den Bundesdurchschnitt, der sich zwischen dem oberen Wert (Hamburg) und dem unteren Wert (Schleswig-Holstein) bewegen muss.

Würde man nun, was der Gesetzgeber in der Reform des GKV-WSG vorgesehen hatte, einen bundesdurchschnittlichen Vergütungssatz einführen, so kann dieser nur zentral als Einheitspreis festgelegt werden. Als Konsequenz daraus ergäbe sich, dass die Preise und die Versorgung in Hamburg mutmaßlich schrumpfen würde, insbesondere auch deswegen, weil aufgrund der angenommenen Kostenstrukturen, die für Hamburg mit der Kostenkurve K2 im rechten 
Teil der Abbildung dargestellt sind, die Kosten für einige Krankenhäuser nicht mehr abgedeckt werden könnten. Umgedreht würde in Schleswig-Holstein die Angebotskapazität ansteigen, da nun in Schleswig-Holstein die Krankenhäuser nicht nur ihre Kosten abdecken, sondern auch noch im Durchschnitt Überschüsse erzielen könnten. In diesem Falle kann man konstatieren, dass ein bundesweiter Durchschnittspreis zu einer Unterversorgung in Hamburg, zu einer Überversorgung in Schleswig-Holstein und zu einer Fehlversorgung im Bundesdurchschnitt führen würde. Die weiteren Konsequenzen, die sich daraus ergeben, wollen wir in den folgenden Punkten noch detaillierter behandeln.

Abbildung 2: Dezentrale Preisdifferenzierung vs. zentrale Einheitspreise

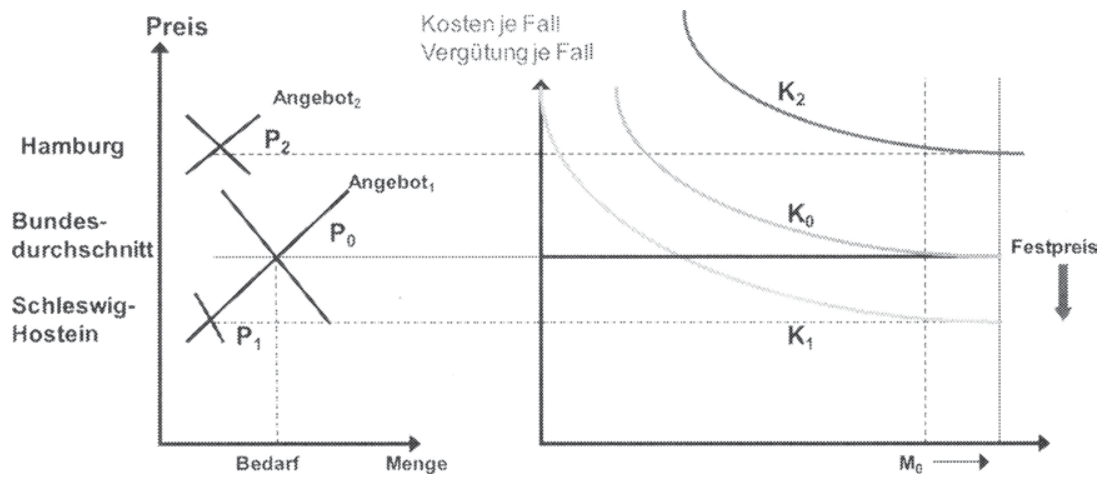

Quelle: Neubauer, G., Pfister, F.: Preisbildung, Stuttgart 2007

\subsection{Konsequenzen zentraler Einheitspreise}

Wie wir schon oben angeführt haben, führen zentrale Einheitspreise klassischerweise zu Über-, Unter- und Fehlversorgung, da sie im Durchschnitt zwar stimmen mögen, aber nirgendwo die konkrete Versorgungssituation widerspiegeln. Insbesondere, da die Kapazitäten im Gesundheitssektor sich nicht rasch verändern lassen, würde dies bei einer zentralen Festlegung der Vergütung die oben beschriebenen Konsequenzen nach sich ziehen. Mutmaßlich würde dies wiederum dazu führen, dass die Preissetzungsbehörde nun versuchen würde, der Fehlallokation durch $\mathrm{Zu}$ - und Abschläge gegenzusteuern. Insgesamt würde dies zu einem bürokratischen Mehraufwand führen. 
Schließlich ist mit Ausweichreaktionen in einem sonstigen wettbewerblichen Umfeld zu rechnen. So würden Krankenhäuser in der „Hochkostenregion“ vermehrt Privatpatienten und Selbstzahler bevorzugen, während in der „Niedrigkostenregion" die Krankenkassen, die ja nun einem Anbietermarkt gegenüberstehen, Rabatte durchsetzen könnten. Es käme also indirekt gleichwohl zu einer Flexibilisierung der Vergütung, trotz staatlicher Einheitspreise.

Ferner ist bei einem an sich wettbewerblichen Umfeld und staatlichen Einheitspreisen mit einem vermehrten Qualitätswettbewerb zu rechnen. Häuser mit hohen Kosten werden versuchen, die hohen Kosten durch höhere Qualität zu rechtfertigen.

\subsection{Konsequenzen von dezentralen Wettbewerbspreisen}

Dezentrale Wettbewerbspreise passen sich jeweils den Vor-Ort-VorsorgungsSituationen an. Im Ergebnis führt eine solche Preisdifferenzierung dazu, dass Patienten und Anbieter jeweils durch ihre Ausweichreaktionen zu einer Angleichung der unterschiedlichen Preise führen würden. Gerade in einem Großraum, wie es Hamburg und Schleswig-Holstein darstellen, käme es zu vermehrten Patientenwanderungen aus dem Hochpreisbereich Hamburg in den Niedrigpreisbereich Schleswig-Holstein. Umgedreht würden Anbieter aus dem Niedrigpreisbereich Schleswig-Holstein vermehrt in den Bereich Hamburg hinein tendieren. Es käme so mittelfristig zu einer dynamischen Angleichung der Preise über die entsprechenden Marktreaktionen. Dass es nie zu einer Preiseinheitlichkeit in einem dynamischen Markt kommen kann, ist bekannt. Nicht nur medizinischorganisatorische und medizinisch-technische Innovationen verschieben ständig die Lage, sondern auch die relativ langsame Kapazitätsanpassung im Gesundheitssektor verzögert eine schnelle Anpassung.

\subsection{Selektives Kontrahieren als Einstieg in den dezentralen Preiswettbewerb}

Um die Preisdifferenzen, die sich durch Wettbewerbspreise ergeben, auch gezielt ausschöpfen zu können, ist es notwendig, dass sich entsprechend die Nachfrage zu dem Ort der günstigeren Preise bewegt. Hier treten als Nachfrager die Krankenkassen auf, die quasi als Agenten der Patienten im Markt agieren und für ihre Versicherten die günstigsten Preiskonditionen vereinbaren wollen. Über das selektive Kontrahieren kommt es so zu einer Flexibilisierung und Dezentralisierung der Preisbildung. 
Für selektive Einzelverträge der Krankenkassen sind Ausschreibungen zwar möglich, aber weniger zweckmäßig, da sich nur das elektive Behandlungsspektrum einbeziehen lässt. Krankenkassen sollten stattdessen mit den Krankenhäusern den Basisfallwert individuell verhandeln und so einen Vertrag für das gesamte Leistungsspektrum des einzelnen Krankenhauses schließen. Freilich setzt dies voraus, dass einzelne Krankenkassen, anders als heute, mit einzelnen Krankenhäusern gezielt separate Verträge abschließen dürfen.

Selektives Kontrahieren im Krankenhausbereich könnte faktisch so ablaufen, dass zunächst - wie bisher - die Krankenkassen gemeinschaftlich und einheitlich mit den Landeskrankenhausgesellschaften den Basisfallwert vereinbaren. Danach wird allen Krankenkassen eingeräumt, selektiv mit einzelnen Krankenhäusern abweichende Vereinbarungen zu treffen. Die Krankenhäuser ihrerseits werden hierbei nur zustimmen, wenn sie Vorteile für sich erzielen. Damit taucht die Frage auf: Wie gut können Krankenkassen ihre Versicherten im Krankheitsfall steuern?

Die Patientensteuerung durch die Krankenkassen hat, wie wir heute sehen, enge Grenzen. Nachweislich reagieren Patienten auf monetäre Anreize, indem etwa im ambulanten Bereich die Praxisgebühr erlassen wird. Auch im Krankenhausbereich vermuten wir eine nicht unbeträchtliche Preiselastizität. Um diese ausschöpfen zu können, ist es einerseits notwendig, den Versicherten vermehrt spezielle Krankenhaustarife anzubieten, bei denen der Versicherte immer dann, wenn er sich verpflichtet selektive Vertragskrankenhäuser bevorzugt aufzusuchen, einen Bonus erhält. Andererseits wäre eine weitergehende Maßnahme, dass Patienten sich generell, oder auch nur optional, prozentual an den Krankenhauskosten beteiligen und so ein direktes Interesse an kostengünstiger Versorgung haben. Die heutige Praxis, dass Patienten in allen Krankenhäusern jeweils pro Tag 10 Euro an „Versorgungsgebühr“ zu entrichten haben, führt zu dem konträren Effekt: Patienten bevorzugen hochwertige Krankenhäuser, da sie für 10 Euro mehr Patientensicherheit versprechen.

Für eine optionale oder auch obligatorische Kostenbeteiligung der Patienten spricht, dass es genügt, wenn nur wenige Patienten sich preiselastisch verhalten - wir schätzen, dass schon 15 Prozent ausreichen -, um die Leistungserbringer, insbesondere die Krankenhäuser, zu einem veränderten Verhalten anzuregen. Schließlich ist die Kapazitätsauslastung der Krankenhäuser heute das wichtigste Instrument, um Kostendeckung, oder sogar Überschüsse zu erzielen. Für nieder- 
gelassene Ärzte ist dieses Instrument nicht ganz so durchgreifend, dürfte aber trotzdem auch Erfolg versprechen.

Freilich ist eine Kostenbeteiligung der Patienten nur sinnvoll, wenn die Leistungserbringer bei einer vermehrten Inanspruchnahme durch Patienten nicht durch einen Vergütungsabschlag bestraft werden. Hier erweist sich einmal mehr, dass die derzeitige Budgetierung der Leistungserbringer und der Bestrafung von Mehrleistungen durch Preisabschläge wettbewerbsfeindlich ist, ja den Leistungswettbewerb auf den Kopf stellt.

\section{Ausblick: Wie wirkt der Zusatzbeitrag?}

Der Zusatzbeitrag, so wie er jetzt (2010/11) nach dem GKV-FinG festgelegt ist, spricht dafür, dass über den Zusatzbeitrag wieder ein dezentraler, wettbewerblicher Anreiz in den Finanzierungsmarkt (vgl. Abb. 1) implantiert wird. Dies hat zur Folge, dass die Krankenkassen ihrerseits verstärkt über selektives Kontrahieren diesen Wettbewerbsimpuls in den Vertragsmarkt übertragen. Würden die Krankenkassen auch auf der anderen Seite die Chancen nutzen, ihren Versicherten verstärkt Kostenbeteiligungsoptionen anzubieten, so würde auch im Leistungsmarkt der Wettbewerb intensiviert, und somit insgesamt das System wettbewerblicher ausgerichtet. $\mathrm{Ob}$ es dazu kommt, hängt freilich nicht zuletzt davon $\mathrm{ab}$, wie die nächste Bundesregierung mit dem Instrument „Zusatzbeitrag“ umgehen wird.

\section{Literatur}

Neubauer, G.: Gesundheitspolitik im Interesse der Versicherten: Strukturreform oder Systemwechsel, in: Die BKK 09/2002, S. 375-382

Neubauer, G.: Festpreise gefährden Versorgung, in: GesundheitsNachrichten 02/2007, S. 6

Neubauer, G.: Von der staatlichen Angebotsplanung zur wettbewerblichen Nachfragesteuerung, in: Die Zukunft der Krankenhaus-Finanzierung, Dieter M. Kampe / Karl-Heinz Bächstädt (Hrsg.), 2007, S. 56-73 
Neubauer, G., Pfister, F.: Preisbildung bei ambulant und stationär erbrachten Gesundheitsleistungen, in: Jahrbücher für Nationalökonomie und Statistik, Gesundheitsökonomie, W. Franz et al. (Hrsg.), Stuttgart 2007, S. 621-635

Neubauer, G., Beivers, A.: Die Prinzipien der Sozialen Marktwirtschaft als Kompass zur Neuordnung der Gesundheitsversorgung in Deutschland: Das Beispiel der vertragsärztlichen Versorgung, in: Anwendungsorientierte Marktwirtschaftslehre und Neue politische Ökonomie, Lothar Funk (Hrsg.), Eckhard Knappe zum 65. Geburtstag, Marburg 2008, S. 371-392

Neubauer, G., Pfister, F.: Der Gesundheitsfonds - Eine gesundheitsökonomische Beurteilung, in: Die Krankenversicherung 10/2008, IKK-Bundesverband (Hrsg), Berlin, S. 256-260 
Eberhard Wille and Klaus Knabner - 978-3-631-75550-1 Downloaded from PubFactory at 01/11/2019 03:39:43AM via free access 


\section{Zum Stellenwert dezentraler Lösungen aus der Sicht eines Bundeslandes}

\section{Manfred Zach}

\section{Ausgangspunkt: Das GKV-Wettbewerbsstärkungsgesetz}

Kein gesundheitspolitisches Gesetz seit dem Gesundheitsstrukturgesetz von 1992 hat die gesundheitspolitische Landschaft so verändert wie das GKVWettbewerbsstärkungsgesetz (GKV-WSG), dessen Kernelemente Organisationsreform, Gesundheitsfonds, Einheitlicher Beitragssatz, Morbi-RSA und Honorarreform zwischen 2007 und 2009 sukzessive in Kraft traten. Im Unterschied zur 1992 eingeleiteten Gesundheitsreform, die eine neue GKV-Wettbewerbsordnung mit freier Kassenwahl und Risikostrukturausgleich schuf, schränkt das GKV-WSG jedoch entgegen seiner euphemistischen Bezeichnung den Wettbewerb um eine bestmögliche Gesundheitsversorgung deutlich ein. Es bevormundet die gesetzlichen Krankenkassen in ihrem finanziellen und gestalterischen Handlungsspielraum, standardisiert die medizinische Regelversorgung auf Durchschnittsniveau und konzentriert maßgebliche gesundheitspolitische Weichenstellungen in den Händen weniger bundespolitischer Entscheidungsträger.

Ermöglicht wurde dies durch die parlamentarisch suboptimale Konstellation einer Regierungskoalition zweier annähernd gleich starker Parteien. Während die SPD die Chance nutzte, das von ihr dominierte Bundesgesundheitsministerium zum eigentlichen Machtzentrum der gesetzlichen Krankenversicherung auszubauen, beschränkte sich die Union um des Koalitionsfriedens willen darauf, eigene gesundheitspolitische Optionen für eine Legislaturperiode ohne sozialdemokratischen Koalitionspartner offen zu halten. In der Zwischenzeit sorgte ein straffes Politikmanagement der Parteizentralen dafür, dass auch aus den Bundesländern keine Fundamentalkritik an einer immer offener staatlichzentralistisch auftretenden Gesundheitspolitik erfolgte. Alle dagegen vorgebrachten fachlichen und ordnungspolitischen Argumente prallten an dieser parteipolitischen Phalanx ab. Selten hat ein Gesetzeswerk in der Fachöffentlichkeit so viel Kritik erfahren, zugleich aber auch deren Ohnmacht offenbart, wenn die politischen Würfel gefallen sind. 


\section{Versorgungspolitisches Durcheinander}

Negative Auswirkungen hatte der mit dem GKV-WSG eingeleitete Paradigmenwechsel vor allem in Bundesländern, die überdurchschnittliche Versorgungs- und Vergütungsniveaus aufwiesen. Die Ungewissheit über ihre künftige finanzielle Lage veranlasste viele Krankenkassen, regionale Sonderleistungen einzustellen. Dies führte z.B. bei chronisch Schmerzkranken, bei Dialyse- und Schlaganfallpatienten zu einem fühlbaren Verlust an Lebensqualität. Gleichzeitig mussten Versicherte, die vor dem 1. Januar 2009 einen günstigeren Versicherungsbeitrag als den nun geltenden einheitlichen Beitragssatz bezahlt hatten, tiefer in die Tasche greifen. Auch im Sprechzimmer ihres Arztes bekamen sie geballten Unmut zu hören, denn ein Orientierungspunktwert von 3,5 Cent bedeutete für Ärzte, deren regional ausgehandelter Punktwert durchschnittlich 3,8 Cent und für ausbudgetierte Einzelleistungen bis zu 5 Cent betragen hatte, ein kräftiges Minus in der Einkommensbilanz.

Nach anfänglicher ,Schockstarre' versuchten die Betroffenen, die schlimmsten Folgen der gesetzlichen Nivellierungen abzumildern. Die Kassenärztliche Vereinigung (KV) Baden-Württemberg spannte einen finanziellen Rettungsschirm auf, der die reformbedingten Honorarverluste je Arztpraxis auf maximal $5 \%$ begrenzte. Der Erweiterte Bewertungsausschuss, das maßgebliche Selbstverwaltungsgremium auf Bundesebene zur Umsetzung der Honorarreform, sanktionierte diese eigenmächtige Maßnahme und schob eine Fülle weiterer Korrekturbeschlüsse nach, welche die Schieflage, in die das Vergütungssystem geraten war, nur noch deutlicher machten. Keinen Anlass zur Klage hatten dagegen die Kassenärztlichen Vereinigungen im Osten und Norden Deutschlands. Ihre Mitglieder konnten sich über sprunghafte Einkommenszuwächse von 20 Prozent und mehr freuen.

Auch bei den gesetzlichen Krankenkassen löste die Sorge um die finanzielle Zukunft hektische Aktivitäten aus. Vor allem die zahlenmäßig größte und regionalwirtschaftlich tief verwurzelte Kassenart der Betriebskrankenkassen suchte Zuflucht in Fusionen mit starken Partnern. Für die Länder bedeutete das: Immer weniger Kassen blieben landesunmittelbar und unterstanden ihrer Rechtsaufsicht. Damit verringerte sich auch der landespolitische Spielraum in Gesundheitsfragen. Zugleich bekam das tradierte kollektivvertragliche Versorgungssystem durch selektive Behandlungs- und Rabattverträge von Kassen mit hausärztlichen Vereinigungen bzw. Arzneimittelherstellern tiefe Risse. Es ist kein Zufall, 
dass in beiden Bereichen die AOK Baden-Württemberg entscheidende Schrittmacherdienste leistete. Im Südwesten Deutschlands schlug die Reform besonders heftig auf, hier regte sich deshalb auch am entschiedensten Widerstand gegen die ,Berliner Politik‘.

\section{Der politische Handlungsdruck wächst}

Der wachsenden Unzufriedenheit mit der Gesundheitsreform konnten sich auch die Landespolitiken nicht entziehen. Der bloße Verweis auf die Übermacht des Bundes genügte nicht, machte er doch zugleich die eigene Ohnmacht deutlich. Ungeachtet partei- und verteilungspolitischer Differenzen trafen sich die Länder daher in der gemeinsamen Überzeugung, der fortschreitenden Aushöhlung ihrer Kompetenzen durch den Bund Einhalt gebieten zu müssen. Auf der 82. Gesundheitsministerkonferenz in Erfurt beschlossen sie einstimmig die Einsetzung einer Arbeitsgruppe auf Staatssekretärsebene zur „Erhaltung der Ländergestaltungsmöglichkeiten in der Gesundheitspolitik“" . Unter Federführung der Länder Sachsen und Bremen legte die Arbeitsgruppe im Mai 2010 einen Forderungskatalog an den Bund vor, den sich die am 1. Juli 2010 in Hannover tagende 83. Gesundheitsministerkonferenz der Länder - wiederum einstimmig - zu eigen machte $^{2}$.

Kernpunkte des Forderungskatalogs waren das Verlangen nach mehr Einflussmöglichkeiten der Länder auf die vertragsärztliche Bedarfsplanung und auf landespolitisch bedeutsame Versorgungsverträge der Krankenkassen sowie eine Änderung der Zuständigkeitsaufteilung für soziale Versicherungsträger zwischen Bund und Ländern. Im Verlauf der Beratungen wurden diese Forderungen sogar in einen ausformulierten Gesetzestext gegossen - ein ungewöhnliches Verfahren, weil Gesetzesinitiativen verfassungsrechtlich nicht durch Fachministergremien, sondern nur von Landesregierungen in den Bundesrat eingebracht werden können. ${ }^{3}$ Weitere einstimmig gefasste Beschlüsse der 83 . Gesundheitsministerkonferenz betrafen die Gewährleistung einer flächendeckenden medizinischen Grundversorgung und die Sicherstellung der hausärztlichen Versorgung.

\footnotetext{
${ }^{1}$ Vgl. Beschlüsse der 82. GMK vom 24./25.06.2009, TOP 5.5. - Frühere Initiativen, z.B. eine 2004 eingesetzte Projektgruppe der Arbeits- und Sozialministerkonferenz „Föderalismus und gesetzliche Kranken- und Pflegeversicherung“, waren politisch folgenlos geblieben.

${ }^{2}$ Vgl. Beschlüsse der 83. GMK vom 01.07.2010, TOP 5.1.

${ }^{3}$ Vgl. Umlaufbeschluss der 83. Gesundheitsministerkonferenz 2010, 12/2010 - Anlage -.
} 
Parallel dazu setzte die 86. Arbeits- und Sozialministerkonferenz der Länder im November 2009 eine Arbeitsgruppe auf Staatssekretärsebene mit dem Auftrag ein, Vorschläge zu einer „unter föderalen Gesichtspunkte sachgerechten Aufteilung der Aufgaben der Aufsichts- und Prüfbehörden des Bundes und der Länder“ zu entwickeln ${ }^{4}$. Die Arbeitsgruppe legte ein Jahr später ihren Abschlussbericht vor. In ihm wird mehrheitlich dafür votiert, die Aufsichtszuständigkeit über die Sozialversicherungsträger solange bei den Ländern zu belassen, bis sich der Zuständigkeitsbereich des Trägers auf das gesamte Bundesgebiet erstreckt, sofern sich die Länder auf ein aufsichtsführendes Land verständigen. Dieser Vorschlag wurde auf der 87. Arbeits- und Sozialministerkonferenz Ende November 2010 einstimmig angenommen ${ }^{5}$.

So viel Demonstration föderaler Einigkeit in gesundheitspolitischen und sozialversicherungsrechtlichen Belangen war bis dahin selten. Natürlich steckte dahinter ein handfestes politisches Kalkül. Zum einen sollte dem Druck der um die Sicherstellung einer flächendeckenden medizinischen Versorgung besorgten Bürger, Kommunalpolitiker und Ärzte mit dem Nachweis entschlossenen Handelns begegnet werden. Zum anderen wurde dem Bund signalisiert, dass er die Zentralisierungsschraube überdreht habe. Dies allerdings hatten die Regierungsparteien CDU, CSU und FDP in ihrem im Oktober 2009 geschlossenen Koalitionsvertrag, der an mehreren Stellen ein Bekenntnis zu mehr Regionalität in der gesetzlichen Krankenversicherung ablegt, selbst schon eingeräumt ${ }^{6}$.

Die eigentliche Triebfeder der neu entdeckten föderalen Gemeinsamkeit dürfte demnach der nicht wegzuleugnende demographische Sprengsatz sein, der im Osten Deutschlands zu spürbaren, im Westen zu drohenden Engpässen in der medizinischen Versorgung führt. Das vorhandene gesetzliche und planerische Instrumentarium reicht nicht mehr aus, um die Gesundheitsversorgung einer alternden Bevölkerung flächendeckend, d.h. auch in dünn besiedelten Räumen, dauerhaft zu gewährleisten. Dies gibt dem Vorgehen der Länder eine über Partikularinteressen hinausreichende gesamtpolitische Legitimation.

${ }^{4}$ Vgl. Beschlüsse der 86. Arbeits- und Sozialministerkonferenz vom 25./26.11.2009, TOP 5.13 .

${ }^{5} \mathrm{Vgl}$. Beschlüsse der 87. Arbeits- und Sozialministerkonferenz 2010 vom 24./25.11.2010, TOP 5.8 (Abschlussbericht als Anlage beigefügt).

${ }^{6}$ Näheres dazu S. 49. 


\section{Kernpunkt Bedarfsplanung}

Kernpunkt der Länderinitiative ist eine Weiterentwicklung der in ihren Grundzügen seit dem Gesundheitsstrukturgesetz 1993 unverändert gebliebenen Bedarfsplanung für die vertragsärztliche Versorgung. Wesentliche Vorgaben dieser in $\S \S 99 \mathrm{ff}$. SGB V geregelten Bedarfsplanung bedürfen der Anpassung an heutige Verhältnisse.

Dazu gehört die überholte Bezugnahme auf Raumordnungskriterien (Stadtund Landkreise als Planungsbereiche) ebenso wie arztgruppenbezogene Verhältniszahlen zur Feststellung von Über- oder Unterversorgung. Regionstypen, die einst in Anlehnung an das Raumgliederungsmodell des Bundesamtes für Bauwesen und Raumordnung entwickelt worden sind, und starre Bevölkerungsrichtwerte geben den dynamischen Wandel in der ärztlichen Versorgung, der Demographie und der Morbiditätsstruktur einer Region nur unzureichend wieder. Unberücksichtigt bleiben bei dieser statischen Betrachtungsweise auch strukturelle Veränderungen in der Ärzteschaft selbst, wie der wachsende Anteil von Frauen und angestellten Ärzten an der ambulanten Medizin oder die zunehmende personelle Verschränkung von stationärer und ambulanter Versorgung.

Die Forderung der 83. Gesundheitsministerkonferenz nach einer flexiblen, kleinräumigen, sektorenübergreifenden und morbiditätsbezogenen Bedarfsplanung sollte daher politisch durchsetzbar sein. Im Gemeinsamen Bundesausschusses (GBA), der die Richtlinien für die Bedarfsplanung festlegt ${ }^{7}$, werden seit längerem intensive Diskussionen über deren bedarfsgerechte Weiterentwicklung geführt ${ }^{8}$. Die Länder selbst sind allerdings nicht in diesem Gremium vertreten, obwohl sie und noch mehr die Kommunen von seinen Beschlüssen direkt betroffen sind. Sie fordern daher, künftig an den Beratungen des GBAUnterausschusses Bedarfsplanung beteiligt zu werden.

Ähnliches gilt für die Landesausschüsse der Ärzte und Krankenkassen, in denen nach den Vorgaben der Bedarfsplanungsrichtlinien die arztgruppenbezogene

\footnotetext{
${ }^{7}$ Vgl. die neu gefasste „Richtlinie des Gemeinsamen Bundesausschusses über die Bedarfsplanung sowie die Maßstäbe zur Feststellung von Überversorgung und Unterversorgung in der vertragsärztlichen Versorgung" vom 15.02.2007, Bundesanzeiger Nr.64 (S. 3954) vom 31.03.2007.

${ }^{8}$ Dies ist einer - nicht veröffentlichten - Stellungnahme des GBA zu den Beschlüssen der GMK vom 01.07.2010 zu entnehmen.
} 
Über- oder Unterversorgung eines Planungsbezirks festgestellt wird. Auch hier haben Länder und Kommunen kein Mitberatungsrecht. Dabei ist die Frage, ob in einer Gemeinde ein neuer Arztsitz zugelassen wird, schon jetzt für die Gesundheitsversorgung im ländlichen Raum von großer Bedeutung. Dies wird sich noch steigern, weil in der Krankenhausplanung, für welche die Länder zuständig sind, seit längerem ein Trend zur Schließung oder Umwidmung kleinerer Krankenhäuser zu beobachten ist. Nur Konzepte für eine aufeinander abgestimmte ambulante und stationäre Versorgung ergeben in Zukunft noch sinnvolle regionale Lösungen. Konsequenterweise halten die Länder deshalb auch die Bildung eines neuen, Sektoren übergreifenden Gremiums (,Gemeinsamer Landesausschuss') zur Koordinierung der ambulanten und stationären Bedarfsplanung für erforderlich - selbstverständlich mit ihrer Beteiligung.

Mehr regionaler Spielraum in der Bedarfsplanung, politische Mitwirkung in den Entscheidungsgremien der Selbstverwaltung auf Bundes- und Landesebene - schon dieses Ansinnen muss dem Bund und den Spitzengremien der gemeinsamen Selbstverwaltung wie eine Kampfansage an tradierte Besitzstände vorkommen. Doch der GMK-Beschluss vom 1. Juli 2010 enthält noch weitere föderale Provokationen.

So verlangen die Gesundheitsminister, dass den Aufsichtsbehörden der Länder künftig alle Verträge zwischen Kassen (also auch Verträge bundesunmittelbarer Kassen) und Leistungserbringern mit Auswirkungen auf das landesbezogene Versorgungsgeschehen vorzulegen seien. Auch müsse die Landesaufsicht mit Blick auf die Bedarfsplanung ein Beanstandungsrecht, erforderlichenfalls sogar ein Initiativrecht für neue Verträge haben. Schließlich: Die grundgesetzlich festgelegte Verwaltungszuständigkeit von Bund und Ländern für soziale Versicherungsträger soll um eine Klausel ergänzt werden, die den Bund ermächtigt, abweichende Regelungen zugunsten der Länder auch unterhalb der verfassungsändernden Schwelle vornehmen zu können.

\section{Reaktionen}

Es verwundert nicht, dass diese geballte Attacke von sechzehn Bundesländern für Aufsehen und auch für politischen Unmut sorgte. In der Fachpresse war von „Kampfansage pur: Länder wollen alles regeln“ die Rede ${ }^{9}$, Bundesgremien wie der GKV-Spitzenverband antworteten mit Stellungnahmen, in denen die meisten

\footnotetext{
${ }^{9}$ Dienst für Gesellschaftspolitik 43 - 10, S. 2.
} 
GMK-Vorschläge als nicht zielführend oder sogar rechtswidrig verworfen wur$\operatorname{den}^{10}$.

Politisch geschmeidig reagierte dagegen Bundesgesundheitsminister Dr. Rösler. Er kündigte für 2011 ein Gesetz zur Sicherstellung der ärztlichen Versorgung (Arbeitstitel: Versorgungsgesetz) an und lud die Gesundheitsminister der Länder ein, mit ihm darüber zu diskutieren. $\mathrm{Da}$ die Länder sich vom Bund nicht einfach zitieren lassen wollten, vereinbarten sie ihrerseits eine Sonder-GMK in Berlin und luden den Bundesgesundheitsminister ein, mit ihnen über die GMKBeschlüsse vom Juli zu diskutieren. Das Treffen kam am 25.10.2010 zustande, doch wer auf konkrete Ergebnisse gehofft hatte, sah sich getäuscht. Der Bundesgesundheitsminister brachte ein Papier mit, in dem Absichtserklärungen zur Weiterentwicklung der Bedarfsplanung, der Sicherstellung und der ärztlichen Nachwuchsförderung enthalten waren, auf die konkreten Forderungen der GMK jedoch mit keinem Wort eingegangen wurde. So mussten es die Länder letztlich schon als Erfolg verbuchen, dass ihnen Dr. Rösler die Mitwirkung in einer gemeinsamen Kommission anbot, in der zur Vorbereitung des Versorgungsgesetzes die Themen Bedarfsplanung, Sicherstellung der ärztlichen Versorgung und Förderung von Medizinstudierenden auf der Basis seines Papiers vertieft behandelt werden sollen.

Damit war der Rahmen abgesteckt, über den der Bund zu diskutieren bereit war. Eine Neuaufteilung der Zuständigkeit für soziale Versicherungsträger zwischen Bund und Ländern oder erweiterte Kompetenzen im Vertragsbereich gehörten ersichtlich nicht dazu ${ }^{11}$.

\section{Rechtliche Bewertung der Länderforderungen}

Das Verlangen der Länder nach mehr Mitsprache wird politisch meist damit begründet, dass die gesundheitliche Betreuung der Bevölkerung vor Ort, also in Städten, Gemeinden und Landkreisen, erfolgt. Ob die damit zweifellos verbundene politische Verantwortung auch konkrete Beteiligungsrechte an der Ausgestaltung der Versorgung auslöst, ist freilich umstritten. Das Grundgesetz trifft insoweit keine explizite Aussage. Jedoch besitzt der Bund eine umfassende Re-

\footnotetext{
${ }^{10} \mathrm{Vgl}$. Stellungnahme des GKV-Spitzenverbandes zur Amtschefkonferenz vom 22.09.2010 in Hannover.

11 Letzteres haben die Länder auf der konstituierenden Sitzung der Kommission am 20.01.2011 in Wiesbaden bei der Auflistung der Arbeitsschwerpunkte allerdings wieder eingebracht.
} 
gelungskompetenz für die Sozialversicherung (Art. 74 Abs. 1 Nr. 12 GG), aufgrund derer er u.a. das Vertragsarztrecht und die Bedarfsplanung bundeseinheitlich normiert hat. Entgegen der allgemeinen Kompetenzzuweisung des Art. 83 GG obliegt den Ländern auch nicht die Ausführung sozialversicherungsrechtlicher Bestimmungen, sondern dies ist zuvörderst Aufgabe der öffentlichrechtlichen Körperschaften der Selbstverwaltung.

Länder und Kommunen verweisen demgegenüber auf ihre Pflicht zur Daseinsvorsorge, die immanenter Bestandteil des Sozialstaatsgebotes (Art. 20 GG) und der verfassungsrechtlich garantierten kommunalen Selbstverwaltung (Art. 28 Abs. 2 GG) sei. Auch die Rechtsnatur und der Geltungsbereich des von der Verwaltungsrechtslehre entwickelten Instituts der Daseinsvorsorge sind allerdings umstritten; Verfassungsrang dürfte ihr kaum zukommen. Insofern spricht mehr dafür, im Gesundheitsbereich trotz seiner politischen Bedeutung nicht von einer originären sondern von einer derivativen Rechtsposition der Länder auszugehen. Sie greift dort, wo der Bund Regelungslücken offen lässt oder, wie in der Krankenhausplanung, den Ländern ausdrücklich Aufgaben übertragen worden sind. Im Umkehrschluss bedeutet dies, dass es dem Bund unbenommen bleibt, Befugnisse wieder an sich zu ziehen, wenn es ihm politisch opportun erscheint. Etwas anderes könnte aufgrund der Föderalismusreform allenfalls dann gelten, wenn der Bund in einem Gesetz, das nicht der Zustimmung des Bundesrates bedarf, Verfahrensregelungen für die Landesebene getroffen hat. Eine solche nicht ,abweichungsfeste' bundesgesetzliche Regelung kann ein Land durch eigene Vorgaben ersetzen. Dies ist im gesundheitspolitischen Bereich bislang aber noch nicht geschehen.

Auch andere Aspekte des GMK-Beschlusses sind rechtlich umstritten. So könnte eine Beteiligung der Länder an Beratungen des Gemeinsamen Bundesausschusses dessen Status als Einrichtung der Selbstverwaltung beeinträchtigen. Dies wäre jedenfalls dann anzunehmen, wenn der Bund, der im Ausschuss bislang ebenfalls nicht vertreten ist, das gleiche Recht für sich reklamieren würde. Weniger eindeutig stellt sich die Situation bei den Landesausschüssen dar. Zwar würde auch hier bei einer Beteiligung des Landes an den Sitzungen des Landesausschusses von Kassenärztlicher Vereinigung und Kassenverbänden der unmittelbare Staatseinfluss zunehmen. Doch lässt sich insoweit mit einem unmittelbaren Sachzusammenhang argumentieren, denn zwischen ambulanter und stationärer Versorgungsplanung, für die das Land zuständig ist, ergeben sich immer mehr sachnotwendige Schnittstellen. 
Die angestrebte stärkere Einbindung der Länder in das regionale Vertragsgeschehen stößt vor allem bei bundesweit tätigen Kassen auf Ablehnung. Sie befürchten Wettbewerbsnachteile, wenn sie ihre Verträge mit Leistungserbringern künftig nicht nur dem Bundesversicherungsamt, sondern auch den Landesaufsichten vorlegen müssten. Bei Verträgen, die von Gesetzes wegen gemeinsam und einheitlich auf Landesebene abzuschließen sind, ist das allerdings heute schon der Fall. Auch wird man den Ländern kaum verwehren können, über Verträge, die für das landesbezogene Versorgungsgeschehen wichtig sind, unterrichtet sein zu wollen. Die Frage ist, ob dieser legitime Informationsbedarf zu einem Beanstandungs- und Initiativrecht führen muss, mit dem in die Vertragsgestaltung von Kassen eingegriffen werden kann, die nicht der Landesaufsicht unterliegen. Kritiker sehen darin eine unzulässige Beschneidung der Vertragsfreiheit bundesunmittelbarer Kassen und eine verfassungsrechtlich bedenkliche Vermischung getrennter Aufsichtsbefugnisse des Bundes und der Länder.

Die vorgeschlagene Grundgesetzänderung ist weniger ein juristisches denn ein realpolitisches Problem. Rechtlich lassen sich mehrere Varianten denken, um die Aufsichtsbefugnisse der Länder über fusionierte Kassen zu erweitern ${ }^{12}$. Faktisch ist aber bislang nicht ersichtlich, was den Bund zu solch einem Schritt veranlassen könnte. Dazu müsste die Solidarität der Länder weit über gemeinsame Fachministerbeschlüsse hinaus reichen. Denn dies ist letztlich die Gretchenfrage der gesamten Regionalisierungsdebatte: Wie weit reicht die Solidarität der Länder wirklich?

\section{Wie weit reicht die Solidarität der Länder?}

Auch wenn es den Ländern gelungen ist, mit ihren Forderungen bundesweit Aufmerksamkeit zu erregen, darf nicht verkannt werden, dass es sich dabei lediglich um den kleinsten gemeinsamen Nenner handelte, auf den man sich verständigen konnte. Kernbereiche der Gesundheitsreform 2007 wie Gesundheitsfonds, einheitlicher Beitragssatz, Morbi-RSA, Honorarreform blieben von vornherein ausgeklammert. Hier gibt es nach wie vor tiefe Interessengegensätze, die allesamt mit Geld zu tun haben.

So gehört es aus Sicht wirtschaftsstarker Bundesländer wie BadenWürttemberg und Bayern zu den strukturellen Ungerechtigkeiten der Reform,

${ }^{12}$ Vgl. z.B. den Abschlussbericht der ASMK-Projektgruppe ,Föderalismus und gesetzliche Kranken- und Pflegeversicherung', 2005. 
dass der Gesundheitsfonds die überdurchschnittliche Ertragskraft ihrer Kassen vollständig abschöpft, was wegen der höheren Ausgabenniveaus in diesen Ländern zur finanziellen Unterdeckung von landesunmittelbaren Kassen und damit zu Abstrichen bei der medizinischen Versorgung führen kann. Aus Sicht der neuen Bundesländer ist dieser Ausgleichsmechanismus dagegen nötig, um Sonderbelastungen, die aus dem geringeren Durchschnittsverdienst und dem höherem Durchschnittsalter der Bevölkerung resultieren, auszugleichen. Mehr Regionalisierung heißt also für die einen tendenziell Leistungsverbesserung, weil mehr Geld im Land verbleibt, die anderen befürchten dagegen Qualitätseinbußen, wenn weniger Geld umverteilt werden kann.

Derselbe Gegensatz zeigt sich bei der Bewertung von Zusatzbeiträgen. Da diese kassenindividuell festgesetzt werden und nicht in den Gesundheitsfonds fließen, werden sie in wirtschaftsstarken Ländern als dezentrales Finanzierungsinstrument positiv beurteilt, wirtschaftsschwache Länder dagegen befürchten sinkende Fondszuweisungen. Die Entscheidung der Bundesregierung ${ }^{13}$, den allgemeinen Beitragssatz gesetzlich festzuschreiben und unvermeidbare Ausgabensteigerungen künftig durch einkommensunabhängige Zusatzbeiträge finanzieren zu lassen, bedeutet in der Tat eine Abkehr vom vollständigen Finanzausgleichssystem des Gesundheitsfonds. Dass Zusatzbeiträge ein schlechtes Image haben und deshalb von den Kassen bislang so weit als möglich vermieden werden, steht auf einem anderen Blatt.

Ein Musterbeispiel dafür, wie schmal die Plattform gemeinsamer Länderinteressen ist, liefert die vertragsärztliche Vergütung. Trotz zweistelliger Honorarsteigerungen zwischen 2008 und 2009 in zehn von sechzehn Kassenärztlichen Vereinigungen gelang es nicht, einen interregionalen Ausgleichstransfer zugunsten jener zwei oder drei KVen zu organisieren, die von dem neuen Vergütungssystem überproportional betroffen waren. Auch Übergangslösungen waren innerhalb der Kassenärztlichen Bundesvereinigung nicht durchsetzen. Es bedurfte des politischen Drucks des Bundes (die Bundeskanzlerin selbst hatte sich dafür verbürgt, dass keine $\mathrm{KV}$ durch die Honorarreform Geld einbüßen werde), um für die ,Verlierer-KVen' eine etwas bessere Vergütungsverteilung in $2011 \mathrm{zu}$ erreichen. Die 80 Millionen Euro, die ,asymmetrisch' nach Baden-Württemberg fließen, nehmen sich allerdings gegenüber den 2009 außerhalb des Landes verteilten 3,5 Milliarden Euro Honorarzuwachs immer noch recht bescheiden aus. Und

${ }^{13}$ Kabinettsbeschluss vom 22.09.2010 zum GKV-Finanzierungsgesetz. 
bezahlt werden sie ohnehin von den Kassen im Land, denn an der bundesweiten Gesamtvergütung änderte sich nichts.

Dies zeigt: Wenn es um Geld geht, setzen die Länder eher auf Konfrontation gegeneinander als auf Solidarität miteinander. Das macht es dem Bund leicht, gemäß den eigenen Interessen zu agieren. Er hat es in der Hand, die Regionalisierungsdebatte voranzutreiben oder sie zu ins Leere laufen zu lassen. Gegenwärtig hat es den Anschein, als sei er zu Zugeständnissen bei der Weiterentwicklung der Bedarfsplanung, der Sicherstellung der ärztlichen Versorgung und der Aus- und Weiterbildung von Ärzten bereit. Andere, für die regionale Gesundheitsversorgung ebenso wichtige Instrumente wie Verträge zwischen Kassen und Leistungserbringern zur hausarztzentrierten Versorgung oder Rabattverträge mit Arzneimittelherstellern wurden dagegen gesetzlich erschwert, obwohl solche Selektivverträge durchaus als regionales Gegengewicht gegen zuviel Zentralismus in der Gesundheitspolitik gelten können.

Von welchen ordnungspolitischen Leitlinien sich der Bund beim Thema Dezentralisierung leiten lässt, ist deshalb im Einzelfall schwer zu prognostizieren. Eher hilft ein Blick auf Aussagen, die CDU, CSU und FDP im Oktober 2009 in ihrer Koalitionsvereinbarung hierzu getroffen haben.

\section{Das ,Regionalisierungs- Pflichtenheft' der Bundesregierung}

Der Koalitionsvertrag für die 17. Legislaturperiode enthält im Abschnitt Gesundheit und Pflege erstaunlich viele Ankündigungen mit Regionalbezug. Krankenversicherungen sollen ,genügend Spielraum erhalten, um regionalen Besonderheiten gerecht zu werden“ (S. 85). Vertragsärztliche Leistungen sollen ,in einem einfachen, verständlichen Vergütungssystem abgebildet werden, bei dem regionale Besonderheiten Berücksichtigung finden“ (S. 88). „Langfristig wird das bestehende Ausgleichssystem überführt in eine Ordnung mit mehr Beitragsautonomie, regionalen Differenzierungsmöglichkeiten und einkommensunabhängigen Arbeitnehmerbeiträgen, die sozial ausgeglichen werden“ (S. 86). „Um der gemeinsamen Verantwortung für regionale Bedürfnisse gerecht $\mathrm{zu}$ werden, sollen fachliche Einwirkungsmöglichkeiten der Länder geprüft werden“ (S. 88).

Die Einführung einkommensunabhängiger Zusatzbeiträge ohne soziale Begrenzung durch das GKV-Finanzierungsgesetz erfüllt das Versprechen von mehr Beitragsautonomie. Regionalen Besonderheiten können Kassen aber erst dann 
gerecht werden, wenn ihnen die dafür notwendigen Mittel zur Verfügung stehen. Eine Möglichkeit wäre, Zusatzbeiträge regional differenzieren zu dürfen. Dies wäre gleichzeitig ein Beitrag zu einer fair ausgestalteten Wettbewerbsordnung zwischen regionalen und überregionalen Kassen.

Ein weiterer Ansatzpunkt für mehr regionale Differenzierungsmöglichkeiten im „Ausgleichssystem“ ist der Risikostrukturausgleich. Die gegenwärtige Einteilung der Versicherten in drei Risikogruppen (Alter/Geschlecht, Erwerbsminderung, hierarchisierte Zuordnung zu 80 Krankheiten) lässt regionale Kostenstrukturen außer Acht, obwohl diese für die Krankenkassen ein ebenfalls nicht unbeträchtlicher Risikofaktor sind. Baden-Württemberg und Bayern fordern deshalb seit langem die Einführung eines Regionalfaktors im RSA, um bei den RSA-Zuweisungen kassenseitige Unter- und Überdeckungen abzufedern. Regionale Abweichungen von den bundesdurchschnittlichen Lebenshaltungskosten oder auch Krankheitsausgaben könnten in halber Höhe als $\mathrm{Zu}$ - oder Abschlag an die jeweiligen Krankenkassen weitergereicht werden. Mit einer solchen Regelung könnte die Bundesregierung den Morbi-RSA zielgenauer machen und den regionalen Handlungsspielraum von Kassen mit Ausgabenunterdeckung stärken.

Noch nicht umgesetzt ist die Ankündigung einer vertragsärztlichen Honorarreform, „,bei der regionale Besonderheiten Berücksichtigung finden“. Da die neue Honorarordnung „einfach und verständlich“ sein soll, ist es mit einigen marginalen Korrekturen am jetzigen, hoch komplexen Vergütungssystem kaum getan. Vielmehr muss die Landesebene, auf der bis zum Inkraftreten des GKVWSG Krankenkassen und Kassenärztliche Vereinigungen die Gesamtvergütung und die außerbudgetären Leistungen detailliert verhandeln konnten, wieder mehr Gestaltungsspielraum erhalten. Vorgaben der Kassenärztliche Bundesvereinigung und des Spitzenverbandes Bund der Krankenkassen sollten sich auf das für ein funktionierendes Gesamtsystem unerlässliche Minimum (Einheitlicher Bewertungsmaßstab für ärztliche Leistungen, Grundsätze für die Mengensteuerung, Festlegung der bundesweiten Gesamtvergütung) beschränken.

\section{Fazit}

Das Thema Regionalisierung hat derzeit politisch Konjunktur. Dies ist einerseits erklärbar, andererseits aber auch erstaunlich. Erklärbar ist es durch die Schockwellen, welche die letzte große Gesundheitsreform auslöste und die zu einer Diskreditierung zentralstaatlicher Bürokratiemodelle führten. Unter diesem Ein- 
druck räumte die Koalitionsvereinbarung der jetzigen Bundesregierung dezentralen Lösungen einen hohen Stellenwert ein. Erstaunlich ist, wie wenig die Länder aus dieser historisch günstigen Situation bislang gemacht haben. Ihre Beschlüsse vom Juli und November 2010 sind nur ein kleinster gemeinsamer Nenner; sie stellen die gesundheitspolitische Zentralisierung an keiner Stelle wirklich in Frage.

So ergibt sich das politisch eigenartige Faktum, dass sogar die Koalitionsvereinbarung der Bundesregierung den Regionalisierungshorizont weiter steckt als die Länder selbst. Daran wird sich solange nichts ändern, als unter den Ländern kein Grundkonsens herrscht, wie viel Regionalisierung überhaupt gewollt ist. Der Bund kann daher weitgehend selbst bestimmen, in welchem Umfang er Kompetenzen abgeben will. Dies ist unter föderalen Gesichtspunkten wenig befriedigend, entspricht aber dem politischen Kräftespiel und ist im Übrigen durch die verfassungsrechtliche Position des Bundes gedeckt.

Regionalität in großem Stil wird es folglich aller Voraussicht nach nicht geben. Denkbar und wahrscheinlich sind eine Fülle kleinerer Korrekturen, die den politischen Gestaltungsspielraum bei der ärztlichen Versorgung vor allem im ländlichen Raum erweitern. Das Zeitfenster dafür wird sich im Wesentlichen auf die Vorbereitungen zum Versorgungsgesetz im Jahr 2011 beschränken. Gespannt darf man sein, ob der Bund in diesem Zusammenhang die beiden großen gesundheitspolitischen Baustellen ,Reform der ärztlichen Vergütung' und ,Reform des Morbi-RSA' anpackt. In beiden Reformkomplexen steckt wegen der finanzpolitischen Implikationen viel Zündstoff für die regionale Gesundheitsversorgung. Konsensuale Lösungen mit allen Ländern erscheinen zurzeit fast ausgeschlossen. Es wird sich zeigen, ob die Bundesregierung gewillt ist, notfalls auch Widerstände zu überwinden. Sieben Landtagswahlen in 2011 sind dabei mit zu bedenken. 
Eberhard Wille and Klaus Knabner - 978-3-631-75550-1 Downloaded from PubFactory at 01/11/2019 03:39:43AM via free access 


\section{Spielräume für eine Dezentralisierung im Rahmen des Systems Gesund- heitsfonds}

\section{Maximilian Gaßner, Dirk Göpffarth und Rüdiger Wittmann}

Wenn in der öffentlichen Diskussion von Dezentralisierung, Regionalisierung oder Föderalisierung der Finanzierung der gesetzlichen Krankenversicherung gesprochen wird, verbergen sich dahinter oft unterschiedliche Inhalte, Vorstellungen und Interessen. Klären wir also zunächst - bevor wir auf die technische Umsetzung eingehen - die Begriffe und Interessen und betrachten wir den Rahmen, der durch die Rechtsprechung vorgegeben wird.

\section{Definitionen und Differenzierungen}

\subsection{Zentralisierung, Dekonzentrierung, Dezentralisierung, Selbstverwaltung}

Im Verwaltungs- und Staatsorganisationsrecht spricht man von Zentralisierung, wenn eine Organisation nicht nur hinsichtlich der Rechtmäßigkeit, sondern auch hinsichtlich der Zweckmäßigkeit ihrer Tätigkeit an negative und positive Weisungen gebunden ist. ${ }^{1}$

Von der Dezentralisierung ist zunächst die Dekonzentrierung zu unterscheiden. Dekonzentriert ist eine Organisation, wenn und soweit ihre weisungsabhängigen Glieder eine eigene Entscheidungsprärogative haben. ${ }^{2}$

Sie ist dezentralisiert, wenn und soweit ihre Glieder, Organe und Ämter nicht einer totalen Zweckmäßigkeitsaufsicht unterliegen, sondern nur einer Rechts ${ }^{3}$ oder allenfalls einer gesetzlich begrenzten Zweckmäßigkeitsaufsicht. ${ }^{4}$ Auf dezentrale Glieder und Organe kann nur auf Grund eines Gesetzes oder ermächtig-

\footnotetext{
${ }^{1}$ Hans J. Wolff, VerwR II, 3. Aufl. $§ 77$ I a; die nachfolgenden Definitionen beruhen auf den dortigen Erläuterungen.

${ }^{2}$ z.B. das Bundesversicherungsamt als selbstständige Bundesoberbehörde im Geschäftsbereich des Bundesministerium für Arbeit und Soziales. Im Rahmen seiner aufsichtsrechtlichen Tätigkeit unterliegt es nur allgemeinen Weisungen der Bundesministerien, dagegen keinen Weisungen im Einzelfall.

${ }^{3}$ z. B. die Träger der Sozialversicherung, § 29 Abs. 1, Abs. 3, §87 Abs. 1 SGB IV.

${ }^{4}$ z.B. $\S 87$ Abs. 2 SGB IV im Bereich der Prävention der Unfallversicherungsträger.
} 
ter Rechtsverordnungen eingewirkt werden. Soweit das nicht geschehen ist, tragen sie selbst die rechtliche und politische Verantwortung für ihre Maßnahmen. ${ }^{5}$

Dezentralisierte Verwaltung bedeutet meist, aber nicht notwendig, auch eine (körperschaftliche) Selbstverwaltung. Diese ist die dezentralisierte Verwaltung eigener Angelegenheiten eines unterstaatlichen Trägers öffentlicher Verwaltung im eigenen Namen und auf eigene Kosten. ${ }^{6}$

\subsection{Teilung der Souveränität auf Bund und Länder}

Von der verwaltungsorganisationsrechtlichen Dezentrierung und Dezentralisierung ist die generelle Teilung der Souveränität des Staates in Bund und Länder, wie es dem bundesstaatlichen Konzept - Art. 20 Abs. 1, 79 Abs. 3 - des Grundgesetzes entspricht, zu unterscheiden. Hier wurde nicht von dem Souverän ein Teil seiner Zuständigkeiten auf den Gebieten der Gesetzgebung, Verwaltung oder Rechtsprechung übertragen, sondern diese Kompetenzen waren von Anfang an aufgeteilt auf zwei verschiedene -souveräne- Ebenen. ${ }^{7}$ Das Bund/Länder- Verhältnis ist nicht statisch. Wenn hier die Länder mehr Dezentralisierung bzw. mehr Regionalisierung fordern, versuchen sie vom Kuchen der Staatlichkeit ein größeres Stück zu Lasten der Staatlichkeit des Bundes zu bekommen. So fordert aktuell die 83. Gesundheitsministerkonferenz 2010 sowie die 87. Arbeits- und Sozialministerkonferenz 2010 eine Änderung des Art. 87 Abs. 2 GG. Danach sollen als bundesunmittelbare Körperschaften des öffentlichen Rechts nur noch diejenigen Sozialversicherungsträger geführt werden, deren Zuständigkeitsbereich sich auf das Gebiet aller Länder erstreckt. Die anderen Versicherungsträger sollen in der Hand der Länder bleiben, es sei denn die Länder einigen sich auf kein aufsichtsführendes Land. Bei Vereinigungen nach dem 31.12.2010 wollen die Länder die Landesunmittelbarkeit des neuen Sozialversicherungsträgers, wenn auch nur ein landesunmittelbarer Sozialversicherungsträger daran beteiligt ist. Konkret: Würde eine der großen Ersatzkassen mit einer kleinen saarländischen Betriebskrankenkasse fusionieren, hätte das Saarland die Rechtsaufsicht über die neue bundesweit agierende Kasse. Mit diesem

\footnotetext{
${ }^{5} \S 29$ Abs. 3 SGB IV.

${ }^{6}$ z.B. die Städte und Gemeinden gem. Art. 28 Abs. 2 GG; aber auch die Sozialversicherungsträger gem. §29 Abs. 1 SGB IV, jedoch ohne eine verfassungsrechtliche Absicherung, BVerfG vom 09.06.2004, NZS 2005 S. 139.

${ }^{7}$ Die Hoheitsgewalt ist so aufgeteilt, dass jeder Teil gegenüber dem anderen einen selbständigen Teilbereich der Hoheitsgewalt innehat, Grzeszick in Maunz/Dürig, Komm. z. GG, Art. 20 Rdn. 38.
} 
Vorschlag würde der vom Gesetzgeber gewollte, bundesweite Wettbewerb der Krankenkassen noch mehr behindert, als er es bereits heute durch die 16 verschiedenen Länderaufsichten ist. Im Übrigen erscheint es fraglich, ob eine funktional dem Bund zuzuordnende Körperschaft dem Land unterstellt werden kann, ohne damit gegen das zentrale und nicht änderbare (Art. 79 Abs. 3 GG) Bundesstaatsprinzip zu verstoßen.

\subsection{Unitarismus/ Föderalismus}

Das Begriffspaar Unitarismus/ Föderalismus bezeichnet die in einem Bundesstaat möglichen verfassungsrechtlichen Organisationsprinzipien der Stärkung der Gliedstaaten auf Kosten des Bundes - Föderalismus - bzw. der Stärkung des Bundes auf Kosten der Gliedstaaten - Unitarismus -.$^{8}$ Damit wird im Wesentlichen die oben (1.2) angesprochene Teilung der Souveränität beschrieben. ${ }^{9}$

\subsection{Regionalisierung}

Spricht man von Regionalisierung, so wird dieser Begriff oft mit dem Begriff der Dezentralisierung oder des Föderalismus gleichgesetzt.

Im ökonomischen Bereich geht es um die Betrachtung, wo und wie Güter und Dienstleistungen erbracht und ausgetauscht werden. Hier schwingt der Unterschied zwischen Markt- und Planwirtschaft mit. Die Marktwirtschaft lebt von der Vorstellung des dezentralen Austauschs von Gütern und Dienstleistungen. Anders die Planwirtschaft. Hier wird - idealtypisch- von einer zentralen Stelle die Versorgung der Mitglieder der Gesellschaft mit Gütern und Dienstleistungen geplant. Taucht hier die Forderung nach Regionalisierung oder Dezentralisierung auf, so ist das mit der Vorstellung verbunden, die Organisation der Versorgung der Versicherten auf regionaler Ebene zu bewerkstelligen, also örtlich nicht weit von ihrem Wohnsitz entfernt.

Auch soweit das Thema Regionalisierung durch die volkswirtschaftliche Brille betrachtet wird, ist festzuhalten, dass der eine oder andere Vertreter einer stärkeren Regionalisierung oder Föderalisierung im Gesundheitswesen kein eigentliches Marktgeschehen will, wo allein die Gesetzmäßigkeiten von Angebot und Nachfrage die Allokation der Güter und Dienstleistungen regeln, sondern es

\footnotetext{
${ }^{8}$ Hans J. Wolff, VerwR II, 3. Aufl., § 77 I 2 d.

9 Zur staatsrechtlichen und staatstheoretischen Unterscheidung, Grzeszick in Maunz/Dürig, Komm. z. GG, Art. 20 Rdn. 18.
} 
geht auch diesen Vertretern mehr oder minder um eine planwirtschaftliche Betrachtung. Der Unterschied liegt allein darin, dass diese Regionalisten oder Föderalisten, um beim Beispiel Deutschland zu bleiben, nicht auf der Ebene Berlin, sondern auf der Ebene München oder Stuttgart „planen und organisieren“ wollen, nach dem Motto „föderal nach außen und zentral nach innen“.

\subsection{Unterschiedlicher räumlicher Bezug}

Zwischen volkswirtschaftlichen Regionalisten oder Dezentralisten und politischen Regionalisten oder Föderalisten besteht jedoch insoweit ein Unterschied, als die politischen Regionalisten in der Kategorie der bestehenden politischen Grenzen der Länder denken. Konkret: Sie wollen die Kompetenzen vom Bund auf die Länder innerhalb der bestehenden Landesgrenzen übertragen.

Die Ökonomen dagegen betrachten die ökonomischen Versorgungszusammenhänge $e^{10}$. In der Lebenswirklichkeit gehen Versorgungsregionen oft über Ländergrenzen hinweg, man denke nur an Regionen wie den Raum Ulm, der Bayern und Baden-Württemberg umfasst oder den nordfränkischen Bereich, in den die Versorgungsregion Frankfurt einstrahlt. Ähnliche Überschreitungen der politischen Ländergrenzen gibt es in den drei Stadtstaaten.

\subsection{Wettbewerbsföderalismus}

Die Sache wird noch komplizierter, wenn man bedenkt, dass auch die politischen Regionalisten oder Föderalisten gerne ihre - politischen - Kompetenzansprüche mit ökonomischen Argumenten abzusichern versuchen. Nach der Philosophie des Wettbewerbsförderalismus oder Konkurrenzföderalismus stehen die Länder als staatliche Körperschaften untereinander in einem - auch ökonomischen - Wettbewerb - Kompetitiver Föderalismus ${ }^{11}$. Parameter hierfür sind großzügigere Investitionsmöglichkeiten für die Industrie, geringere Abgabenbelastungen für die Unternehmen, eine unbürokratische Administration, eine moderne, gut ausgestattete Infrastruktur, ein anspruchsvolles Bildungswesen mit industrienahen Forschungsaktivitäten, qualifizierte, leistungswillige Arbeitskräfte u.s.w.. Dass diese Parameter in dem einen Land besser, in dem anderen Land schlechter seien, liege nicht allein an Naturgesetzlichkeiten, sondern an den dort

\footnotetext{
${ }^{10}$ Jacobs K., G+G 11/2010 S. 14, 15: Die Gleichsetzung von Region und Land ist unbrauchbar.

${ }^{11}$ Seine Wurzeln habe er in sozialwissenschaftlichen Überlegungen und im Bereich der Wirtschaft, so Robbers in BK, Art. 20 Abs. 1 Rdn. 1066-1069.
} 
tätigen Menschen und den Anstrengungen ihrer jeweiligen Landesregierungen. Deshalb müssten die Früchte der Anstrengungen, die höheren Steuer- und Beitragseinnahmen, den Bürgern dieser Länder zugutekommen und dürften ihnen nicht über steuerliche Finanzausgleiche oder über sonstige Ausgleiche im Sozialversicherungssystem, wie z.B. dem Risikostrukturausgleich, wieder genommen werden. ${ }^{12}$ Interessengemäß werden diese Positionen von den wirtschaftlich stärkeren Ländern im Süden Deutschlands vertreten und stoßen dementsprechend auf Skepsis bei den wirtschaftlich schwächeren, insbesondere bei den östlichen Ländern. Dies verwundert nicht. Sollen die Nehmerländer doch überzeugt werden, dass auch sie später mehr haben werden, wenn sie aktuell weniger bekommen. „Geld gegen Hoffnung" verkauft sich in Deutschland schlecht, wie ein Blick in die Aktionärsstruktur des Deutschen Aktienindex beweist. „Geld gegen mehr eigene Anstrengung“" ist nahezu unverkäuflich.

\subsection{Politische Parteien und Regionalisierung}

Nach dem Konzept des Grundgesetzes ist die Staatlichkeit Deutschlands auf Bund und Länder aufgeteilt. Eine Klammer zwischen Bund und Ländern bilden im Wesentlichen die politischen Parteien. Ihre Existenz ist in Art. 21 GG abgesichert. Sämtliche politisch relevanten Parteien sind sowohl auf der Ebene des Bundes als auch auf der Ebene der Länder vertreten. Wenngleich die CSU nur in Bayern zur Wahl antritt, hat sie auch auf Bundesebene politische Ambitionen. Machtpolitisch ist für die Parteien die Bundesebene weitaus bedeutsamer als die Landesebene. Dies führt in der alltäglichen politischen Auseinandersetzung meist zur Zurückstellung der politisch schwächeren Länder- bzw. Regionalinteressen gegenüber den stärkeren Bundesinteressen. Etwas anderes gilt in der politischen Realität allenfalls dann, wenn parteipolitisch starke Persönlichkeiten Mandate auf Länderebene haben und auch die Länder- bzw. Regionalinteressen vertreten (Land geht vor Partei). Freilich ist auch in einer solchen Konstellation immer zu beachten, dass das Landesinteresse nicht isoliert dem Bundesinteresse gegenüber steht, sondern erst innerhalb der 16 Länder koordiniert werden muss. Auch das schwächt im Antagonismus zwischen Bund und Ländern die Position der Länder.

${ }^{12} \mathrm{Gaßner}$ M./ Dürschke J./ Zorzi M., Wettbewerbsföderalismus und Überforderungsschutz in der Sozialversicherung, Zeitschrift für Sozialhilfe und Sozialgesetzbuch, 1998, S. 259 - 267. 


\section{Zur Entstehung der Kassenvielfalt in Deutschland}

Unter den verschiedenen Zweigen der Sozialversicherung hat die gesetzliche Krankenversicherung trotz aller Fusionen bis zum heutigen Tag immer noch die größte Zahl von Versicherungsträgern. Diese große Trägervielfalt rührt von den Anfängen der gesetzlichen Krankenversicherung her. ${ }^{13}$ Der Reichsgesetzgeber ${ }^{14}$ knüpfte Ende des 19. Jahrhunderts an die bereits damals bestehenden dezentralen Einheiten an und schuf nicht eine völlig neue zentrale Struktur, wie dies zum Beispiel in Großbritannien bei Einführung des staatlichen Gesundheitsdienstes nach dem Zweiten Weltkrieg geschehen ist. Die vorhandenen dezentralen Einheiten waren im Wesentlichen durch die Eigeninitiative von Betroffenen oder Verbänden von Betroffenen entstanden; es waren also grundsätzlich keine Gründungen der Landesherren oder der Landesregierungen. Soweit ersichtlich, gab es zum damaligen Zeitpunkt keine ernsthaften kompetenzrechtlichen Auseinandersetzungen zwischen dem Reich und den Ländern in Bezug auf die Gesetzgebung und die Aufsichtskompetenzen.

Der Reichsgesetzgeber hielt nach der dem Gesetz vom 15.06 .1883 beigefügten Begründung die Bildung größerer Kassen nicht für erforderlich, weil das Risiko der Krankheit nicht so groß sei, ,dass es nicht von kleineren Kreisen getragen werden könnte“. Das dürfte in ökonomischer Hinsicht den damals begrenzten medizinischen Therapiemöglichkeiten entsprochen haben. Herzoperationen, Organtransplantationen, Dialyseeinrichtungen und Medikamente bei Bluterkrankheit etc. gab es zum damaligen Zeitpunkt nicht. Kassen mit kleineren Bezirken hätten den Vorteil, dass in einer großen Zahl von Fällen ohne „Erledigung weitläufiger Verhandlungen" sofort Unterstützung geleistet werden könne und die Voraussetzungen des Unterstützungsanspruchs durch Organe festgestellt und überwacht werden könnten, die mit denen in Betracht kommenden Verhältnissen vertraut seien. ${ }^{15}$ Auch auf die Eigenkontrolle der Versicherten zur Korruptionsbekämpfung wurde hingewiesen.

Infolge der Gesetzgebung gab es zu Beginn des 20. Jahrhunderts über 23.000 Krankenkassen. Diese Vielzahl von kleinen und kleinsten Kassen rief - nach

13 Zur historischen Entwicklung vergleiche die Ausführungen im BSG-Urteil vom 22.05.1985, BSGE 58 S. 135f.

${ }^{14}$ Gesetz betreffend die Krankenversicherung der Arbeiter vom 15.06.1883, Reichsgesetzblatt 1883 S. 73-104.

${ }^{15} \mathrm{Zu}$ weiteren Einzelheiten BSG v. 22.05.1985, BSGE 58 S. 135. 
einer längeren politischen Diskussion über die Unübersichtlichkeit und die mangelnde Leistungsfähigkeit kleinster Kassen - wiederum den Gesetzgeber auf den Plan mit der Konsequenz, dass im Jahre 1932 nur noch 6.611 Gesetzliche Krankenkassen und 51 Ersatzkassen, die zusammen rund 18,7 Mio. Versicherte betreuten, bestanden.

Nach dem 2. Weltkrieg setzte sich diese Konsolidierung der Zahl der Kassen fort. Zwischenzeitlich hat sie sich durch verschiedene gesetzgeberische Maßnahmen zum Jahresende 2010 auf 160 zurückentwickelt. Dennoch ist der Konsolidierungsprozess noch nicht abgeschlossen, sondern schreitet - wenngleich mit geringerem Tempo - auch heute noch fort.

Soweit ersichtlich, gab es bis zum 2.Weltkrieg keine ernsthaften kompetenzrechtlichen Auseinandersetzungen zwischen dem Reich und den Ländern, weder in Bezug auf die Gesetzgebungskompetenz, noch in Bezug auf die Aufsichtskompetenzen. Das Reichsversicherungsamt war im Übrigen ursprünglich nur für die Invaliditätsversicherung zuständig. Erst 1913 wurde seine Zuständigkeit auf die Krankenversicherung und nach dem 2. Weltkrieg auf die Knappschaft und die Arbeitslosenversicherung erweitert.

Erst mit dem rapiden Ansteigen des Finanzbedarfs der Gesetzlichen Krankenversicherung nach dem 2. Weltkrieg, verbunden mit der starken Spreizung der Beitragssätze zwischen den einzelnen Kassen und Kassenarten, ergab sich der Bedarf für ein Ausgleichssystem. Dieses knüpfte zuerst an den Ausgaben der Rentner an, später betraf es sämtliche Versicherte. ${ }^{16}$

\section{Verfassungsrechtliche Betrachtung}

Kompetenz- und materiellrechtlich setzten sich sowohl das Bundessozialgericht als auch das Bundesverfassungsgericht mit der Thematik der Legitimation von Ausgleichssystemen in der Gesetzlichen Krankenversicherung auseinander. Zwei Leitentscheidungen seien angeführt:

${ }^{16}$ Zur Entwicklung im Einzelnen vgl. BVerfGE 113, 167 f.; JURIS Rdn. 1 - 33. 
Im Hinblick auf den Gleichheitssatz stellte das BSG in der Entscheidung fest,

- dass das Krankenversicherungssystem als gegliedertes System - Kassenarten, bundes- und landesunmittelbare Kassen -, das mehrere Strukturprinzipien nebeneinander verwende, eher als ein homogenes System eines Ausgleichs bedürfe. Das gelte besonders für Unterschiede in den Beitragssätzen der Krankenkassen, jedenfalls wenn die Sätze erheblich voneinander abweichen, die Versicherten jedoch in vielerlei Hinsicht Gemeinsamkeiten aufwiesen, und gerade solche versicherungsrechtlich bedeutsamer Art (etwa in der Höhe des Grundlohns oder in örtlicher oder beruflicher Beziehung). ${ }^{17}$

- Ungleiche Beitragssätze seien indessen die ,notwendige“ Folge eines gegliederten, d. h. dezentralen Aufbaus der Gesetzlichen Krankenversicherung. Der Gesetzgeber brauche bei seiner Entscheidung nicht allein oder vorrangig die Herstellung der Beitragssatzgleichheit zum Ziel haben, sondern könne im Rahmen seiner Gestaltungsfreiheit auch anderen sachlichen Erwägungen Raum geben und ihnen damit die Einheit der Versicherung und Gleichheit der Beitragssätze bis zu einem gewissen Grade „opfern“. ${ }^{18}$

- Auf der anderen Seite sei aber nicht zu verkennen, dass die Gesetzliche Krankenversicherung trotz ihres dezentralen Aufbaus ein einheitliches System sei. ${ }^{19}$ Ein Ausgleichsbedarf sei am ehesten dort anzuerkennen, wo der hohe Beitragssatz auf Ursachen zurückzuführen sei, zu deren Ausgleich die soziale Krankenversicherung gerade geschaffen worden sei. Das gelte namentlich für Unterschiede bei den Grundlöhnen, bei der Zahl der mitversicherten Angehörigen und in der Risikostruktur. Finde insoweit kein genügender Ausgleich innerhalb des bestehenden dezentralen Krankenversicherungssystems, d.h. bei der einzelnen Kasse, statt, so müsse dem Ausgleichsbedürfnis in anderer Weise Rechnung getragen werden, ohne dass allerdings ein vollständiger Ausgleich gefordert werden könne. ${ }^{20}$

- Nicht in gleichem Maße ausgleichbedürftig seien die Auswirkungen, die eine regional hohe medizinische Versorgung (Überversorgung) für den Beitragssatz habe. Auch der Umstand, dass der Leistungskatalog für die Kassen zwischenzeitlich im Wesentlichen gleich sei, rechtfertige allein noch keinen

\footnotetext{
${ }^{17}$ JURIS Rdn. 31.

18 JURIS Rdn. 36.

19 JURIS Rdn. 37.

20 JURIS Rdn. 44.
} 
Ausgleich zwischen Kassen mit relativ hohen und solchen mit geringeren Leistungsaufwendungen. $^{21}$

- Es gebe schließlich auch Ursachen, die geradezu ,ausgleichsfeindlich“ seien. Hierzu gehörten vor allem hohe Verwaltungskosten, eine zu großzügige Leistungsgewährung seitens der Kassenverwaltung, ungenügende Kontrollen, namentlich was die Wirtschaftlichkeit erbrachten Leistungen betrifft, sowie ein übersteigertes Anspruchsverhalten der Versicherten. ${ }^{22}$

\subsection{Bundesverfassungsgericht vom 18.07.2005, BVerfGE $113 \mathrm{~S} .167 \mathrm{f}$.}

In kompetenzrechtlicher und materiellrechtlicher Sicht hat das Bundesverfassungsgericht mit seiner Entscheidung vom 18.07.2005 zum „alten“ Risikostrukturausgleich die Pflöcke und damit die Handlungsmöglichkeiten für eine Regionalisierung klar und deutlich abgesteckt.

\subsubsection{Kompetenz}

Kompetenzrechtlich hält das Gericht fest, dass

- eine in allen Landesteilen gleich funktionsfähige Sozialversicherung auf der Basis unterschiedlicher Ländergesetze praktisch kaum denkbar sei, so dass die Gleichwertigkeit der Lebensverhältnisse (Art. 72 Abs. 2 GG) entscheidend von einer bundesgesetzlichen Regelung abhänge. Nicht zuletzt die gleichheitsrechtlich gebotene bundesweite Angleichung der Beitragssätze in der Gesetzlichen Krankenversicherung lasse sich mit unterschiedlichen landesrechtlichen Regelungen nicht erreichen. Divergierendes Landesrecht könne auch die Mobilität der Versicherten innerhalb des Bundesgebiets einschränken und für die abführungspflichtigen Unternehmen Handlungsbeschränkungen verursachen. ${ }^{23}$

- Die Kompetenznorm des Art. 74 Abs. 1 Nr. 12 GG und die Bestimmungen der Art. 87 Abs. 2 und Art. 120 Abs. 1 S. 4 GG bildeten ein in sich geschlossenes Regelungssystem für die Sozialversicherung und deren Finanzierung. Diese Bestimmungen würden als speziellere Normen den allgemeinen steuerzentrierten Vorschriften des X. Abschnitts des Grundgesetzes vorgehen. ${ }^{24}$

${ }^{21}$ JURIS Rdn. 45.

22 JURIS Rdn. 46.

23 JURIS Rdn. 90.

24 JURIS Rdn. 93. 
- Die Finanzmittel der landes- und bundesunmittelbaren Krankenkassen den Ländern oder dem Bund zuzuordnen und dem Regelungssystem der Art. 104 a ff. GG zu unterwerfen, würde dagegen das sachliche Gesetzgebungsrecht des Bundes zur Organisation der Sozialversicherung weitgehend entwerten. $^{25}$

\subsection{2 materielle Erwägungen}

Materiell sei,

- die Grundentscheidung des Gesetzgebers für das Gebiet der gesamten Bundesrepublik als räumlicher Bezugsrahmen für den Risikostrukturausgleich ohne Berücksichtigung regional unterschiedlicher Kostenstrukturen im Hinblick auf die wettbewerblichen Ziele sachgerecht gewesen. ${ }^{26}$ Sollten regional unterschiedliche Krankheitshäufigkeiten die Ursache für regionale Ausgabenunterschiede sein, so fordere der Zweck des Solidarausgleichs zwischen Gesunden und Kranken grundsätzlich deren Berücksichtigung. ${ }^{27}$

- Wenn es dagegen, wie angenommen werde, in den einzelnen Regionen ein unterschiedliches Verhalten der Versicherten bei der Nachfrage nach Krankenkassenleistungen gebe und hieraus regional unterschiedliche Ausgabenbelastungen resultieren sollten, dann musste der Gesetzgeber diesen Umstand nicht im Risikostrukturausgleich Rechnung tragen. Auf die Höhe des Einkommens der Mitglieder habe die Krankenkasse keinen Einfluss, wohl aber auf deren Anspruchsverhalten. Beeinflussbare Faktoren könnten und sollten von den Krankenkassen zur Erhöhung von Effizienzen und Wirtschaftlichkeit beeinflusst werden. Dies sei der Sinn des vom Gesetzgeber gewollten Kassenwettbewerbs. ${ }^{28}$

- Soweit regional unterschiedliche Kostenniveaus darauf beruhten, dass es zwischen Stadt und Land Unterschiede im Hinblick auf Versorgungsdichte oder die Qualität der Versorgung (modernere, bessere, teuerere Ausstattung) gebe, sei es gerechtfertigt, dass diejenigen Versicherten, die in den Genuss einer regional besseren Versorgung gelangten die hieraus resultierenden Mehrkosten in Form von höheren Beitragssätzen alleine zu tragen hätten.

${ }^{25}$ JURIS Rdn. 96.

26 JURIS Rdn. 201.

${ }^{27}$ Dabei sei, so Jacobs, K. G+G 11/2010 S. 14, 15, das Schöne am morbiditätsorientierten Risikostrukturausgleich, dass er automatisch regionalisiere.

${ }^{28}$ JURIS Rdn. 205. 
Beitragssatzunterschiede seien verfassungsrechtlich nur dann problematisch, wenn Versicherte trotz gleich hohen Einkommens für gleiche Leistungen unterschiedlich viel zahlen müssten. ${ }^{29}$

- Schließlich seien auch mögliche regionale Wirtschaftlichkeits- und Effizienzunterschiede als Ursache für regionale Kostenunterschiede nicht notwendig ausgleichsrelevant. Dass es etwa Regionen gebe, in denen die Krankenhausbedarfsplanung besser funktioniere als in anderen Landesteilen, musste den Gesetzgeber - unabhängig von einem möglichen Einfluss der Kassen nicht zur Berücksichtigung veranlassen. Ziel sei es, die Wirtschaftlichkeit der Leistungserbringer insgesamt zu verbessern, nicht aber, regionale Unwirtschaftlichkeiten durch Zuweisungen eines höheren Beitragsbedarfs zu subventionieren und damit $z u$ verfestigen. ${ }^{30}$

- Soweit schließlich die Unangemessenheit und Unzumutbarkeit des Risikostrukturausgleichs pauschal mit dessen beträchtlichen finanziellen Auswirkungen bei einzelnen ausgleichsverpflichteten Kassen begründet werde, finde dies im allgemeinen Gleichheitssatz keine Grundlage. Ohne Risikostrukturausgleich würden die Beitragssätze der Krankenkassen weit auseinander liegen. Nach der Rechtsprechung des Bundesverfassungsgerichts bedürfe gerade ein solcher Zustand verfassungsrechtlicher Rechtfertigung, nicht aber das vom Gesetzgeber angestrebte Ziel der gleichmäßigen Verteilung der Solidarlasten. ${ }^{31}$

\subsection{Rechtliches Fazit}

1. Die Verteilung der Finanzmittel der Sozialversicherung hat keinen Bezug zu den Ländern und den Länderhaushalten. Selbst Regionalkassen und die von ihnen verwalteten Beitragsmittel können finanzverfassungsrechtlich nicht als Teil eines Landes oder als Teil der Landeshaushalte betrachtet werden. ${ }^{32}$ Es gibt also kein „bayerisches Geld“ und schon gar nicht ein Geld des Freistaats Bayern in der Krankenversicherung. ${ }^{33}$

\footnotetext{
${ }^{29}$ JURIS Rdn. 206.

30 JURIS Rdn. 207.

31 JURIS Rdn. 253.
}

${ }^{32}$ Deshalb ist auch keine Zustimmungsbedürftigkeit eines Gesetzes gemäß Art. 104a Abs. 4 GG gegeben, wenn dieses landesunmittelbaren Sozialversicherungsträgern Kosten aufbürdet, vgl. Axer P., VSSR 1/ 2010 S. 1, 7.

${ }^{33}$ Insgesamt sei die Sozialversicherung ein besonders deutliches Beispiel für den unitaristischen Charakter des deutschen Föderalismus, so Ebsen I., Föderalismus in der Sozialversicherung, GGW 4/2005 S. 7, 13. Ebenso Axer P., VSSR 1/2010 S. 1, 25, der feststellt, dass der 
2. Da das Beitragsaufkommen der Gesetzlichen Krankenversicherung einer bundesweit organisierten Solidarität unterliegt, würde eine Berücksichtigung regional unterschiedlich hohen Beitragsaufkommens im Ausgleichssystem des Risikostrukturausgleichs im systematischen Widerspruch zu dieser bundesweiten, gleichheitsrechtlich (Art. 3 GG) gebotenen Betrachtung stehen (Widerspruch zur Systemgerechtigkeit des RSA-Systems). Regionale Differenzierungen sind deshalb auf dieser Ebene ausgeschlossen. ${ }^{34}$

3. Regionale Ausgabenunterschiede können vom Gesetzgeber berücksichtigt werden. Eine zwingende Notwendigkeit besteht jedoch nicht. ${ }^{35}$

4. Ausgleichsfeindlich sind aber solche regionalen Kostenunterschiede, die auf einer großzügigeren Leistungsgewährung, ungenügenden Kontrollen der Wirtschaftlichkeit der Leistungserbringung sowie auf einem übersteigerten Anspruchsverhalten der Versicherten beruhen.

\section{Der Blick ins Ausland}

\subsection{Belgien}

Risikostrukturausgleiche sind immer dann notwendig, wenn ein wettbewerbliches Krankenversicherungssystem mit freier Wahl des Versicherers mit sozialpolitisch motivierten Einschränkungen bei der Prämiengestaltung (insbesondere einem Verbot risikoäquivalenter Prämien) verbunden wird. Dementsprechend finden sich auch im europäischen Ausland Risikostrukturausgleiche, soweit dort wettbewerbliche Krankenversicherungssysteme existieren.

In Belgien besteht eine Krankenversicherungspflicht, wobei jeder Bürger die freie Wahl der Krankenkasse hat. ${ }^{36}$ Es gibt rund 100 Krankenkassen, die sich in sechs verschiedenen Kassenarten organisieren. Die Finanzierung folgt aus einem zentralen Gesundheitsfonds, der aus einkommensabhängigen Sozialversicherungsbeiträgen und Steuerzuschüssen finanziert wird. Hinzu kommt ein nicht einkommensbezogener, nicht risikoäquivalenter Zusatzbeitrag, der direkt an die

soziale Bundesstaat den Art. 20 Abs. 1 GG normiere, sich auch nach den Föderalismusreformen als ein unitaristischer Sozialstaat erweise. Letztlich liege dies in dem auf soziale Gleichheit hin ausgerichteten Bundesstaat des Grundgesetzes begründet.

${ }^{34}$ Bundeseinheitliche Gleichheit habe Vorrang vor wettbewerbsfördernder Diversität, Ebsen, a.a.O. S. 13.

${ }^{35}$ Vgl. auch Schmehl, A. S. 1020, in Sodan H., Handbuch des Krankenversicherungsrechts, München 2010.

${ }^{36}$ Die folgende Beschreibung folgt Schokkaert, E./Van de Voorde, C., Belgium: risk adjustment and financial responsibility in a centralised system, Health Policy 65 (2003): 5-19. 
Krankenkasse zu entrichten ist. Die Krankenkassen erhalten risikoadjustierte Zuweisungen aus dem zentralen Gesundheitsfonds.

Der für die Berechnung der Zuweisungen notwendige Risikostrukturausgleich existiert seit dem Jahr 1995. Im Jahr 2008 wurde dieser zu einem morbiditätsorientierten Risikostrukturausgleich ausgebaut. ${ }^{37}$ Insgesamt enthält das heute verwendete Klassifikationsmodell 155 Risikogruppen, davon 40 Alters- und Geschlechtsgruppen, 16 arzneimittelbasierte sowie 66 krankenhausdiagnosebasierte Morbiditätsgruppen und 33 weitere soziodemographische Variablen (Erwerbsminderung, Urbanitätsgrad, Singlehaushalt, Selbständigkeit etc.). Anders als der morbiditätsorientierte Risikostrukturausgleich in Deutschland oder den Niederlanden werden die Diagnosen und Verordnungen in Belgien nicht prospektiv, sondern zeitgleich herangezogen.

Regionalmerkmale werden damit in Belgien auf der Einnahmenseite nicht berücksichtigt. Sowohl die Sozialversicherungsbeiträge wie auch die Zusatzbeiträge sind nicht regional differenziert. Allerdings finden regionale Unterschiede bei den Zuweisungen Berücksichtigung. Dies erfolgt zum einen durch eine (eingeschränkte) Berücksichtigung medizinischer Angebotsstrukturen, vor allem aber über die Berücksichtigung des Urbanitätsgrades (Bevölkerungsdichte und Verstädterung).

\subsection{Niederlande}

In den Niederlanden wurde zum 1. Januar 2006 die Trennung zwischen gesetzlicher und privater Krankenversicherung aufgehoben. ${ }^{38}$ Alle Krankenversicherungen operieren in einem privatrechtlichen Rahmen, allerdings mit umfangreichen staatlichen Regulierungen. Wie in Deutschland und Belgien erfolgt die Finanzierung über einen Gesundheitsfonds, wobei der Finanzierungsanteil des Gesundheitsfonds $50 \%$ beträgt. Die andere Hälfte erfolgt durch nicht einkommensabhängige, nicht risikoäquivalente Prämien, die direkt vom Versicherten an die Krankenversicherung entrichtet werden. Allerdings werden die Prämien von Kindern und Jugendlichen aus Steuermitteln übernommen, zudem existiert ein steuerfinanzierter Sozialausgleich.

\footnotetext{
${ }^{37}$ Van de Voorde, C., Essays on Risk Adjustment in Health Insurance, Diss., Katholieke Universiteit Leuven, 2010.

${ }^{38}$ Die folgende Beschreibung folgt VWS (Niederländisches Ministerium für Gesundheit, Gemeinwohl und Sport), The new care system in the Netherlands, Den Haag o.J.
} 
Zur Berechnung der Zuweisungen aus dem Gesundheitsfonds existiert seit dem Jahr 1991 ein Risikostrukturausgleich, der ursprünglich nur für die gesetzliche Krankenversicherung galt. Die anfänglich berücksichtigten Ausgleichsfaktoren waren Alter, Geschlecht, Art des Einkommens (insbesondere Einkommensersatzleistungen wie Sozialhilfe, Arbeitslosengeld etc.) und Region (Urbanitätsgrad). In den folgenden Jahren wurde der Risikostrukturausgleich kontinuierlich durch zusätzliche Ausgleichsvariablen erweitert: Im Jahr 2002 wurden Pharmakostengruppen (PCG) eingeführt, im Jahr 2004 (stationäre) Diagnosekostengruppen (DCG) und bei der Art des Einkommens zwischen abhängiger Beschäftigung und selbständiger Tätigkeit unterschieden. ${ }^{39}$ Seit dem Jahr 2007 können Versicherte auch zu mehr als einer Pharmakostengruppe gleichzeitig zugeordnet werden; gleichzeitig wurde die Zahl der Pharmakostengruppen von ursprünglich 12 auf inzwischen 23 kontinuierlich erhöht. ${ }^{40} \mathrm{Im}$ Jahr 2008 wurde der sozioökonomische Status des Versicherten als weitere Ausgleichvariable aufgenommen. Die Aufnahme weiterer Variablen (funktionale Gesundheitseinschränkungen, Pflegestatus) wird diskutiert.

Es ist zu berücksichtigen, dass in den Niederlanden der prospektive Risikostrukturausgleich durch weitere sogenannte „ex-post-Ausgleiche“ ergänzt wird. Dabei handelt es sich um einen Risikopool mit einem Schwellenwert von 12.500 Euro und einer Ausgleichsquote von $90 \%$, einem proportionalen Ausgabenausgleich von $35 \%$ der variablen Krankenhausausgaben und $100 \%$ der Fixkosten der Krankenhausbehandlung und einer „Fangnetzregelung“, die greift, wenn die tatsächlichen Ausgaben je Versicherten die Zuweisungen um 35 Euro über- oder unterschreiten und $90 \%$ des Differenzbetrages ausgleicht.

Die Niederlande kennt regionale Unterscheidungen auf der Einnahmenseite. Während zwar der Beitragssatz für die an den Gesundheitsfonds gezahlten Sozialversicherungsbeiträge nicht differenziert ist, dürfen die Krankenkassen ihre Prämien auf Ebene der Provinzen differenzieren. Auch der Risikostrukturausgleich berücksichtigt bei den Zuweisungen regionale Unterschiede. Dabei wird ein sehr aufwändiges Regionalkonzept verwandt, dass sich nicht an den Grenzen von Gebietskörperschaften orientiert. Grundidee ist, dass die Unterschiede zwi-

\footnotetext{
${ }^{39} \mathrm{Vgl}$. Ven de Ven, W./Beck, K./Van de Voorde, C./Wasem, J./Zmora, I., Risk adjustment and risk selecrion in Europe, Health Policy 83 (2007): 162-179.

${ }^{40}$ Vgl. im Folgenden: VWS (Niederländisches Ministerium für Gesundheit, Gemeinwohl und Sport), Beschreibung der Risikostrukturausgleichssystematik des niederländischen Krankenversicherungsgesetzes, Den Haag 2008.
} 
schen den tatsächlichen Ausgaben und den Zuweisungen auf Ebene von Postleitzahlregionen ausgeglichen werden sollen, soweit diese durch regionale Risikofaktoren erklärt werden können. $\mathrm{Zu}$ diesen regionalen Risikofaktoren zählen: der Anteil an Singlehaushalten, die Bevölkerungsdichte, der Anteil an Einwanderern, Abstand zum nächsten Krankenhaus, Abstand zum nächsten Hausarzt sowie die Bettendichte der Krankenhäuser. Anhand dieser Variablen werden die Postleitzahlregionen zu zehn verschiedenen, nicht geographisch zusammenhängenden Regionen zusammengeführt, für die es dann unterschiedlich hohe $\mathrm{Zu}$ weisungen aus dem Gesundheitsfonds gibt. ${ }^{41}$

\subsection{Israel}

Auch in Israel sind Krankenkassen die Träger der Krankenversicherung, wobei eine freie Wahl zwischen vier Krankenkassen besteht. Die Finanzierung erfolgt aus einem zentralen Gesundheitsfonds, der aus Steuermitteln und Sozialversicherungsbeiträgen gespeist wird.

Einen Risikostrukturausgleich zur Mittelverteilung mit der Ausgleichsvariable Alter kennt man in Israel seit dem Jahr 1995. Zusätzliche Zahlungen gibt es für Versicherte mit den folgenden Krankheiten: Dialysestatus, Morbus Gauche, Thalassämie, Hämophilie und AIDS (Shmueli et al. 2003). Seit November 2011 wurden zwei weitere Ausgleichsvariablen eingeführt: Geschlecht und Region (im Sinne von Zentrum vs. Peripherie). ${ }^{42}$ Damit existiert in Israel zwar keine regionale Differenzierung auf der Einnahmenseite, wohl aber neuerdings auf der Zuweisungsseite. Die Regionalvariable als Zentrum-Peripherie-Variable hat den ausdrücklichen Zweck über höhere Zuweisungen an randständige Regionen die dortigen Versorgungsdefizite abzubauen.

\subsection{Schweiz}

Auch in der Schweiz existiert ein Krankenversicherungsmarkt mit Wettbewerb zwischen Krankenkassen, die einem Kontrahierungszwang unterliegen und keine risikoäquivalenten Prämien erheben dürfen. Anders als in Deutschland, Belgien, den Niederlanden oder Israel erfolgt die Finanzierung nicht über einen Gesundheitsfonds. Vielmehr gehen die Prämieneinnahmen - wie in Deutschland

41 Buchner, F., Region and Risk Adjustment, Vortrag bei der Jahrestagung des Risk Adjustment Networks am 17.9.2010 in Berlin.

42 Persönliche Auskunft von Prof. Amir Shmueli, Hebrew University of Jerusalem. 
vor dem Jahr 2009 - direkt an die Krankenkassen, werden aber anschließend im Rahmen eines nachgelagerten Risikostrukturausgleichs umverteilt, wobei es dadurch zu Nettozahlern und Nettoempfängern kommt.

In der Schweiz wurde der Risikoausgleich zwischen den Krankenkassen (in seiner heutige Form) im Jahr 1996 eingeführt. ${ }^{43}$ Hierfür werden getrennt nach Frauen und Männern jeweils 15 Altersklassen gebildet, wobei Kinder und Jugendliche unter 19 Jahren nicht in den Risikoausgleich einbezogen werden. Der Risikoausgleich wird für die Versicherten eines jeden Kantons separat durchgeführt. Vom Jahr 2012 an soll als weitere Ausgleichsvariable die Hospitalisierung im Vorjahr einbezogen werden.

In der Schweiz wurde der Risikostrukturausgleich somit auf kantonaler Ebene eingeführt. Grundidee ist es, finanzielle Transfers zwischen den Kantonen zu unterbinden. Dies stellt die extremste und konsequenteste Form der Einbindung eines Regionalfaktors in den Risikostrukturausgleich dar. Konsequenterweise erfolgt dann auch die Prämienkalkulation auf kantonaler Ebene, wobei auch Differenzierungen unterhalb der Kantonsebene zulässig sind (maximal drei Prämienregionen je Kanton).

\section{Die Konvergenzklausel des $§ 272$ SGB V als Beispiel für eine temporä- re Regionalisierung des Risikostrukturausgleichs}

Mit der Einführung des Gesundheitsfonds am 1. Januar 2009 trat auch in der GKV erstmalig eine Regelung in Kraft, die über ein Regionalmerkmal (das Bundesland des Wohnsitzes der Versicherten) direkt die Mittelausstattung einer Krankenkasse beeinflusste: die in $\S 272$ SGB V verankerte, sogenannte „Konvergenzklausel“. Im Gegensatz zu den übrigen hier diskutierten Modellen handelt es sich bei dieser Regelung nicht um eine auf Dauer angelegte Regionalkomponente, sondern um ein Verfahrenselement, das explizit nur in der Einführungsphase des Gesundheitsfonds gilt.

Konkret soll mit dem als Kompromiss geborenen Verfahren sichergestellt werden, ,dass sich die Belastungen auf Grund der Einführung des Gesundheitsfonds für die in einem Land tätigen Krankenkassen in jährlichen Schritten von jeweils höchstens 100 Millionen Euro aufbauen“. ${ }^{44}$ Die Konvergenzklausel ist somit ein

${ }^{43}$ Vgl. hierfür und im Folgenden Beck, K., Risiko Krankenversicherung, 2. Aufl., Bern 2011. ${ }^{44}$ Vgl. $\$ 272$ Abs. 1 S. 1 SGB V. 
Belastungsausgleich, der die Umverteilungswirkungen durch die Einführung des Gesundheitsfonds und des morbiditätsorientierten Risikostrukturausgleichs auf regionaler Ebene zeitlich entzerrt.

Hintergrund der Übergangsphase war die Antizipation beträchtlicher regionaler Mittelumverteilungen (primär) über drei Mechanismen: Erstens wurde angenommen, dass die Einführung eines zentralen Gesundheitsfonds dazu führen würde, dass die bis dahin verbliebenen Wettbewerbsvorteile von Krankenkassen mit einkommensstarken Mitgliedern nun vollständig entfielen. Während in der alten Systematik lediglich etwa $91 \%$ der Beitragseinnahmen einem Finanzkraftausgleich unterworfen waren, so würde dies im „System Gesundheitsfonds“ für deren Gesamtheit gelten. Übertragen auf eine regionale Betrachtungsebene wurden daher Mittelabflüsse vor allem aus Bundesländern mit einer überdurchschnittlich hohen Einkommensstruktur erwartet. Der zweite Aspekt berührt die parallel mit der Einführung des Gesundheitsfonds umgesetzte Morbiditätsorientierung des Risikostrukturausgleichs, der die Fondszuweisungen aus Regionen mit einer (vormals) günstigen Risikostruktur insbesondere in jene Regionen leitet, in denen eine überdurchschnittlich hohe alters- und geschlechtsstandardisierte Morbidität vorherrscht. Eine dritte Einflussgröße auf die Be- und Entlastungen auf Länderebene wurde in der Einführung eines bundeseinheitlichen Beitragssatzes gesehen. Durch die Reduktion ihrer überdurchschnittlich hohen Beitragssätze auf einen bundeseinheitlichen Wert wurde für einige Länder ein zusätzlicher Finanzverlust befürchtet - denn ehemals überdurchschnittlich hohe Beitragseinnahmen würden mit Inkrafttreten des Fonds nur noch (mehr oder weniger) durchschnittlichen Zuweisungen gegenüberstehen.

Für bestimmte Regionen wurde angenommen, dass sich die drei genannten Effekte zu einer schlagartig eintretenden Veränderung der verfügbaren Mittel in erheblicher Höhe hätten aufaddieren können. Dies aber hätte zu Belastungen geführt, die vor allen Dingen mit unvorhersehbaren Auswirkungen auf der Versorgungsebene verbunden gewesen wären. Insbesondere Bundesländer mit hohen Grundlöhnen, überdurchschnittlich hohen Beitragssätzen, einer gut ausgebauten medizinischen Infrastruktur und einer eher gesunden Versichertenstruktur hatten somit ein begründetes Interesse daran, ,unverhältnismäßige Belastungssprünge auf Grund der Einführung des Gesundheitsfonds zu vermeiden““ ${ }^{45}$

${ }^{45}$ Vgl. BT-Drs. 16/3100, S.91. 
Die Berechnung der länderspezifischen Belastungen gemäß der Konvergenzklausel hatte das Bundesversicherungsamt erstmalig im Herbst 2008 zu berechnen, wobei es ,die Höhe der fortgeschriebenen Einnahmen der Kassen für die in einem Land wohnhaften Versicherten den Zuweisungen aus dem Gesundheitsfonds gegenüber ${ }^{46}$ zu stellen hatte. In der Umsetzung wird dies - grob umrissen - mit den folgenden Berechnungsschritten erreicht, die dem Bundesversicherungsamt detaillierter auch in den $\S \S 33 \mathrm{f}$. der Risikostruktur-Ausgleichsverordnung (RSAV) vorgegeben sind:

Für jedes Bundesland wird zunächst ein fiktiver landesspezifischer Beitragssatz ermittelt. Zur Berechnung werden sowohl die im Jahr 2008 gültigen Beitragssätze und die entsprechenden Mitgliederzahlen der in dem jewiligen Bundesland tätigen Krankenkassen herangezogen. Hieraus lassen sich in einem weiteren Schritt die fiktiven Beitragseinnahmen der 16 virtuellen „Länderkassen“ für das Ausgangsjahr 2008 berechnen.

Um das „Altverfahren“ originalgetreu auf die Landesebene zu projezieren, werden die Beitragseinnahmen der Länderkassen im Anschluss um die Ausgleichsbeträge bereinigt, die sich aus einem regionalisierten Risikostrukturausgleichs- und Risikopoolverfahren (jeweils mit den für 2008 gültigen Ausgestaltungsparametern) ergeben hätten. Es resultieren die „bereinigten Beitragseinnahmen" je Bundesland.

Abschließend werden die bereinigten Einnahmen der Länderkassen auf das jeweilige Vergleichsjahr fortgeschrieben und mit den (ebenfalls auf Länderkassen heruntergebrochenen) Zuweisungen aus dem Gesundheitsfonds verglichen. Übersteigen die „fortgeschriebenen bereinigten“ Beitragseinnahmen in einem Land die Zuweisungen aus dem Gesundheitsfonds, so liegt eine Belastung vor. Übersteigt diese Belastung den (sich jährlich um 100 Millionen Euro erhöhenden) Schwellenwert, so wird die Differenz zum Schwellenwert ausgeglichen.

Mit dem Abschluss des Jahresausgleichs liegt inzwischen die endgültige Berechnung der Konvergenzregelung für das Ausgleichsjahr 2009 vor. Dabei wurden länderspezifische Belastungen über dem für das Jahr festgelegten Schwellenwert von 100 Millionen Euro für fünf Bundesländer ermittelt. Auf dieser Grundlage kam es zu Erhöhungen der Zuweisungen für Versicherte mit Wohnort in Schleswig-Holstein, Hessen, Rheinland-Pfalz, Baden-Württemberg und

${ }^{46}$ Vgl. $§ 267$ Abs. 1 S. 2 SGB V. 
Sachsen-Anhalt (vgl. Abbildung 1). Insgesamt nahmen die aus der Liquiditätsreserve des Fonds zugewiesenen Mittel für den regionalen Belastungsausgleich ein Volumen von 121,3 Millionen Euro an. Dieses unerwartet geringe Verteilungsvolumen der Konvergenzklausel zeigt, dass die ursprünglich befürchteten hohen Belastungssprünge so nicht eingetreten sind.

Abbildung 1: Länderspezifische Be- und Entlastungen im Sinne des $\$ 272$ SGB V (2009)

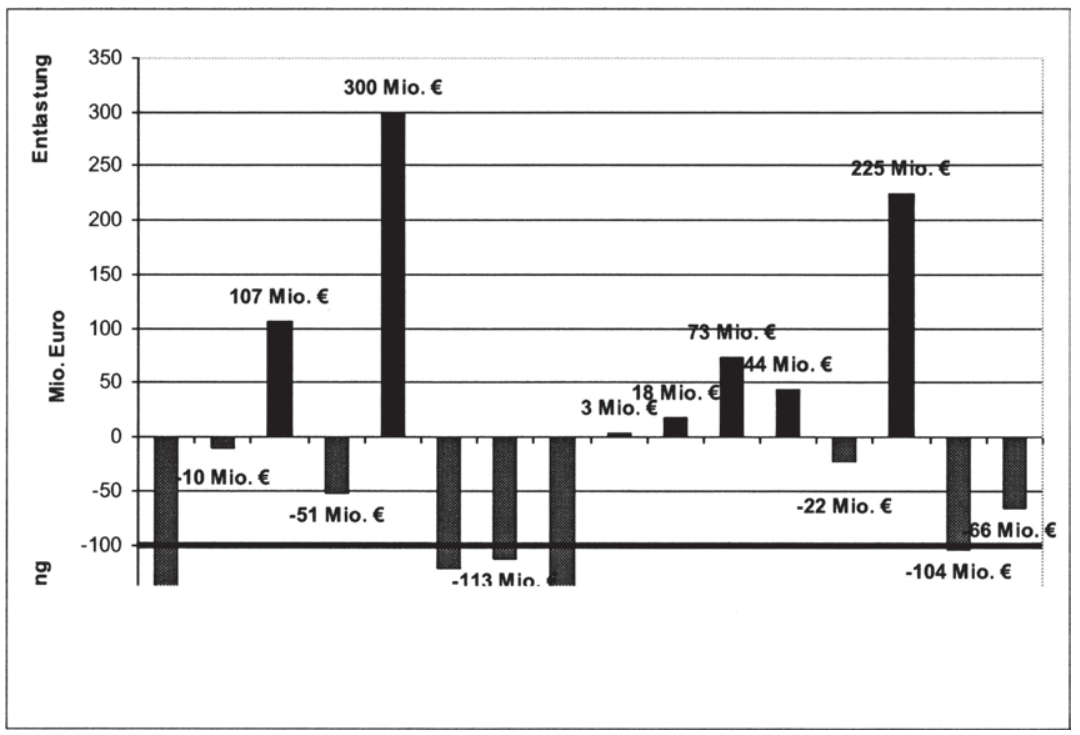

Ein etwas differenzierteres Bild zeigt sich, wenn man die Wirkung des vervollständigten Finanzkraftausgleichs isoliert (vgl. Abbildung 2). Es zeigen sich hier - wenn auch nicht unbedingt in der vor Einführung des Gesundheitsfonds von Kritikern befürchteten Höhe - spürbare Belastungen vor allen Dingen bei Krankenkassen, die in Ländern mit überdurchschnittlich vielen einkommensstarken Mitgliedern tätig sind: Die im besonders grundlohnstarken BadenWürttemberg tätigen Krankenkassen erfahren alleine über die Vervollständigung des Finanzkraftausgleichs eine Belastung in Höhe von 107 Millionen Euro, was im wesentlichen auch der im Rahmen der Konvergenzklausel ausgewiesenen Belastung entspricht. 
Abbildung 2: Auswirkungen der Vervollständigung des Finanzkraftausgleichs

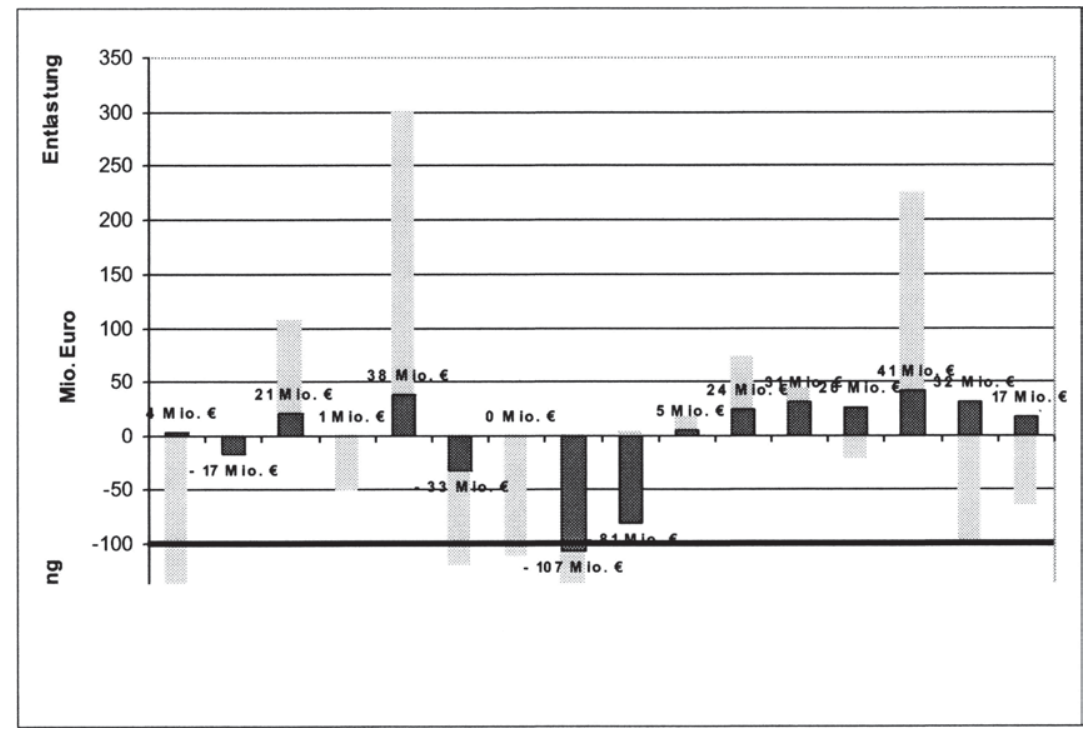

Auch die Einführung des morbiditätsorientierten Risikostrukturausgleichs hat relevante Auswirkungen auf die regionale Verteilung der Mittel. Abbildung 3 stellt die mittleren „Beitragsbedarfe“ der alten Systematik den mittleren ,Zuweisungen" im Gesundheitsfonds für die einzelnen Länder gegenüber. Hier zeigt sich, dass der Morbi-RSA seine stärkste Verteilungswirkung erwartungsgemäß in den Bundesländern entfaltet, die bekanntermaßen eine überdurchschnittlich hohe Krankheitslast aufweisen - insbesondere Krankenkassen mit Tätigkeitsschwerpunkt im Saarland und in Berlin profitieren von der verstärkten Morbiditätsorientierung. Dies erklärt auch, warum es für in Berlin tätige Krankenkassen - anders als zunächst prognostiziert ${ }^{47}$ - keine Mittel aus der Konvergenzklausel gibt.

\footnotetext{
${ }^{47}$ Wasem, J./Buchner, F./Wille, E., Umsetzung und empirische Abschätzung der Übergangsregelung zur Einführung des Gesundheitsfonds, Diskussionsbeitrag aus dem Fachbereich Wirtschaftswissenschaften Universität Duisburg-Essen, Essen 2008, S. 54.
} 
Abbildung 3: Jährliche RSA-Zuweisungen je Versicherten je Bundesland

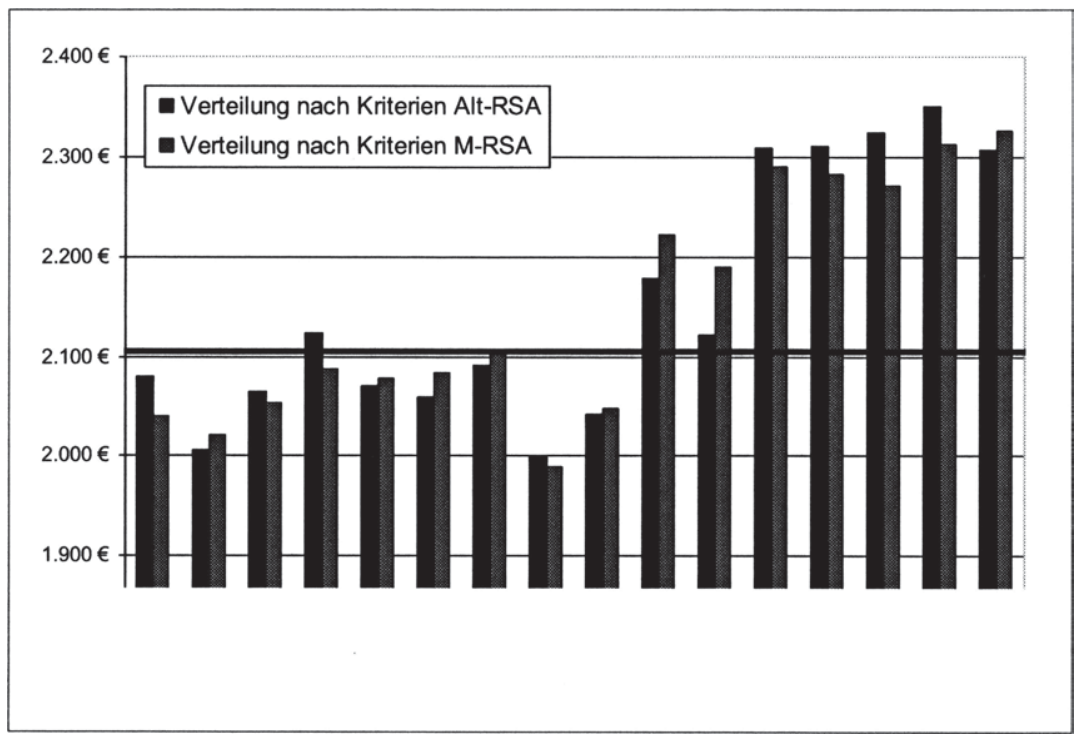

Der dritte Effekt, der sich auf die Ermittlung der Belastungsbeträge auswirkt, folgt aus der Einführung des bundesweit einheitlichen Beitragssatzes. Für Länderkassen, die vor Einführung des Gesundheitsfonds einen Beitragssatz erhoben haben, der über dem Durchschnitt der GKV lag, errechnet sich im Sinne der Konvergenzklausel eine Belastung, da sich für Kassen mit überdurchschnittlichen Beitragssätzen auch relativ hohe Beitragseinnahmen für das Jahr 2008 ergeben. Länder mit vormals unterdurchschnittlichen Beitragssätzen werden dagegen entlastet. In der Sache kann man über diese Interpretationsweise trefflich streiten, da die Mitglieder der entsprechenden Kassen von der Anhebung des einheitlichen Beitragssatzes auf 15,5\% faktisch weniger stark betroffen waren als Beitragszahler, deren Krankenkassen zuvor einen unterdurchschnittlichen Beitragssatz veranschlagt hatten. Es werden somit regionale Be- und Entlastungen ausgewiesen, die sich in dieser Form bei einer ganzheitlicheren Betrachtung überhaupt nicht ergeben haben. Die rechnerische Wirkung des Beitragssatzeffektes verdeutlicht eindrücklich das Beispiel Sachsens: Während die Vervollständigung des Finanzkraftausgleichs in Sachsen zu einer Entlastung von rund 40 Millionen Euro führt, wäre zunächst davon auszugehen, dass die ,Zuweisungsverluste" durch Einführung des Morbi-RSA die Entlastung kompensiert. Tatsächlich wird Sachsen aber im Sinne des § 272 SGB V insgesamt um 225 
Millionen Euro entlastet - eben weil der für die virtuelle sächsische Länderkasse berechnete Beitragssatz (13,8 \%) mit Abstand unter den für die übrigen Länder ermittelten Werte $(14,1 \%$ - 15,2 \%) und somit deutlich unter dem GKV-Schnitt $(14,8 \%)$ liegt.

Ein gravierendes methodisches Problem bei der Messung der regionalen Belastungen im Rahmen der Konvergenzregelung ergibt sich darüber hinaus dadurch, dass ein wesentlicher Effekt der räumlichen Verteilung der Kasseneinnahmen schlichtweg ausgeblendet wird. Die durchgeführten Berechnungen vernachlässigen, dass ein großer Anteil der GKV-Population in bundesweit tätigen Krankenkassen versichert ist, die sowohl in der alten als auch in der neuen Systematik erhebliche interregionale Finanztransfers geleistet haben und noch immer leisten. Denn damals wie heute wurden und werden bei überregional tätigen Kassen die durch ihre Mischkalkulation in bestimmten Regionen entstehenden Überdeckungen in andere Gebiete umgeschichtet, in denen sie zur Versorgung benötigt werden. Dass es sich hierbei um Finanzströme von zum Teil erheblichem Ausmaß handelt, ist in der Vergangenheit mehrfach aufgezeigt worden. ${ }^{48}$ Dass eben diese Transferleistungen bei der Berechnung der Konvergenzklausel nicht berücksichtigt werden, erschwert die korrekte Deutung ihrer Ergebnisse erheblich.

Alles in allem muss somit festgehalten werden, dass die Konvergenzregelung aufgrund ihrer methodischen Eigenheiten nur bedingt dazu geeignet ist, die länderspezifische Umverteilung durch die Einführung des Gesundheitsfonds vollständig darzustellen. Ungeachtet seiner offensichtlichen Mängel stellt sich vor dem Hintergrund der gegenwärtigen Regionalisierungsdebatte dennoch die grundsätzliche Frage, ob das Konvergenzverfahren - zumindest dem Prinzip nach - dazu geeignet wäre, regionale Besonderheiten in der Versorgungs-, Vergütungs- und Morbiditätsstruktur auf Dauer zielführend abzubilden. Diese Frage kann allerdings ohne längeres Zögern verneint werden. Denn Ziel der Regelung nach $\S 272$ SGB V ist lediglich ein Ausgleich fiktiver Belastungen, die sich durch einen Vergleich der gegenwärtigen regionalen Mitteldistribution mit den Verhältnissen zu einem eher zufällig definierten Ausgangspunkt ergeben. Völlig

\footnotetext{
${ }^{48}$ Wasem et al., Umsetzung, a.a.O., S. 72ff.; Schürmann, C. Be- und Entlastungen von Bundesländern und die Folgen für den Wettbewerb - Finanzkraftausgleich und der Transfer bei bundesweit tätigen Krankenkassen und Kassenwettbewerb. In: Repschläger, U. (Hrsg.), BARMER - Gesundheitswesen aktuell 2008, Wuppertal 2008, S. 166ff.; BVA, Regionale Verteilungswirkungen des Risikostrukturausgleichs, Bonn 2004, S. 14.
} 
unbeantwortet bleibt aber, ob zu diesem Ausgangszeitpunkt für die Zielsetzung eines fairen Wettbewerbs der Krankenkassen innerhalb eines bundesweiten Solidarsystems überhaupt eine optimale - oder wenigstens eine annähernd zielgenaue - Verteilung der Mittel im Raum vorlag.

Anders ausgedrückt: Die Vervollständigung des Finanzkraftausgleichs und die Morbiditätsorientierung im Risikostrukturausgleich sind der Rahmen eines solidarischen Wettbewerbs der Krankenkassen. Dieser ist ordnungspolitisch ebenso erwünscht wie die sich hieraus ergebende finanzielle Umverteilung. Zwar erscheint es durchaus legitim, Belastungsspitzen, die sich durch eine tiefgreifende Systemumstellung ergeben können, in einer Übergangsphase abzufedern; eine dauerhafte Festschreibung der bestehenden Regelung aber (etwa über die Fixierung einer konstanten Belastungshöchstgrenze im Vergleich zum Status 2008) würde der eigentlichen ordnungspolitischen Zielsetzung aber diametral entgegenstehen.

\section{Wie könnte ein Regionalmodell im bestehenden morbiditätsorientier- ten Risikostrukturausgleich aussehen?}

6.1 Bei den regionalen Strukturunterschieden sind drei Aspekte zu berücksichtigen

Sollte die Konsequenz aus den Erfahrungen mit der Konvergenzklausel sein, künftig ganz auf regionale Differenzierungen im Risikostrukturausgleich zu verzichten? Das wäre angesichts der Bedeutung der durch die Daten aus der Durchführung aufgezeigten regionalen Strukturunterschiede sicherlich die falsche Konsequenz. Vielmehr sollte es Ziel sein, aus den Erfahrungen der Konvergenzklausel zu lernen und eine sinnvolle und dauerhafte Form der Berücksichtigung regionaler Unterschiede bei den Zuweisungen aus dem Gesundheitsfonds zu finden. Dabei sollte ein solches Regionalmodell stärker in die Rechenlogik des (morbiditätsorientierten) Risikostrukturausgleichs integriert werden und die Rechenbesonderheiten, die die gegenwärtige Konvergenzklausel so schwer interpretierbar machen, vermieden werden.

Bei der Frage, ob regionale Strukturunterschiede im Risikostrukturausgleich berücksichtigt und damit auch ausgeglichen werden sollen, sind drei Aspekte zu berücksichtigen: 
Zum einen stellen regionale Ausgabenunterschiede zwischen den Krankenkassen wichtige und in der Regel nicht beeinflussbare Faktoren dar. Wenn das gegenwärtige System mit einem faktischen Einheitsbeitrag übergeht in ein System mit Preisdifferenzierung über Zusatzbeiträge mit Sozialausgleich, erhält dieses Problem eine erhebliche wettbewerbliche Relevanz. Dann stehen Krankenkassen mit einer regionalen Zusatzbeitragskalkulation im Wettbewerb mit Krankenkassen, die ihre Zusatzbeiträge überregional kalkulieren. ${ }^{49}$ Überregional kalkulierende Krankenkassen haben einen Wettbewerbsvorteil in teuren Regionen, regional kalkulierende Krankenkassen einen Vorteil in günstigeren Regionen. Die offensichtliche Antwort auf diese Frage wäre eine generelle regionale Zusatzbeitragsgestaltung. Will man diesen Weg aber z.B. aus regionalpolitischen Gründen nicht gehen, so stellt sich die Frage, ob ein Ausgleich regionaler Unterschiede im Risikostrukturausgleich eine Alternative zur Stärkung der Funktionalität des Kassenwettbewerbs sein kann.

Der zweite Aspekt bezieht sich auf die Funktionalität des Risikostrukturausgleichs, der die Anreize zur Risikoselektion für Krankenkassen neutralisieren soll. Regionale Über- und Unterdeckungen aus dem Risikostrukturausgleich sind insofern problematisch, da sie Anreize für regionale Selektionsstrategien für Krankenkassen bieten können, wofür es auch schon empirische Belege gibt. ${ }^{50}$ Dies kann sich auch bis in die Versorgung auswirken, da sich aus Sicht einer kühl kalkulierenden Krankenkasse ein besonderes Engagement in der Versorgung in einer unterdeckten Region nicht rechnet. Eine Berücksichtigung regionaler Ausgabenunterschiede erhöht die Zielgenauigkeit der Zuweisungen aus dem Gesundheitsfonds und reduziert etwaige regionale Über- und Unterdeckungen.

Während die ersten beiden Aspekte für eine Berücksichtigung von Regionalmerkmalen im Risikostrukturausgleich sprechen, weist der dritte Aspekt auf mögliche Probleme hin. Grundsätzliche Wirkung von Regionalfaktoren im Risikostrukturausgleich ist eine höhere Mittelausstattung in ausgabenintensiven Regionen und eine geringere Mittelausstattung in weniger ausgabenintensiven $\mathrm{Re}$ gionen. Dies wäre vernünftig, wenn das regionale Ausgabenniveau ein verlässli-

\footnotetext{
${ }^{49} \mathrm{Vgl}$. für das analoge Problem bei der alten Beitragssatzgestaltung Felder, S., Regionalisierung, Risikostrukturausgleich und Wettbewerb in der gesetzlichen Krankenversicherung, Zeitschrift für die gesamte Versicherungswissenschaft 88 (1999): 59-75.

${ }^{50}$ Bauhoff, S., Do Health Plans Risk-Select? An Audit Study on Germany's Social Health Insurance, Journal of Public Economics, in Druck.
} 
cher Indikator für den regionalen Bedarf ist, also die Ausgaben in einer Region höher sind, weil dort mehr Kranke zu versorgen sind. Tatsächlich wird das Ausgabenniveau aber auch angebotsgetrieben sein, z.B. durch zu hohe Bettenzahlen in Krankenhäusern. Wird nun die Mittelausstattung in solchen Regionen erhöht, nimmt der Druck zur Kostenreduzierung (z.B. Bettenabbau) ab. ${ }^{51}$ Umgekehrt würde in unterversorgten Regionen die Unterversorgung festgeschrieben, da weniger Mittel in die Region flössen.

Diese Problematik hat auch das BSG in seinem den RSA herbeiführenden, oben (3.1.) besprochenen Urteil aus dem Jahr 1985 dazu bewogen zu erklären, dass regionale Unterschiede gegenüber Unterschieden in der Finanzkraft, der Zahl der Familienversicherten und in der Risikostruktur der Krankenkassen nicht im gleichen Maßen ausgleichsbedürftig seien, während die Folgen unwirtschaftlichen Verhaltens geradezu ,ausgleichsfeindlich“ "seien. ${ }^{52}$

Wegen dieser Gefahren eines vollständigen Ausgleichs aller regionalen Unterschiede sollte und darf ein Regionalmerkmal im Risikostrukturausgleich nicht vollständig wirken. Aber wie soll das rechte Maß bestimmt werden? Optimalerweise müsste man zunächst untersuchen, inwieweit die beobachteten regionalen Differenzen auf Unterschiede im Versorgungsbedarf und inwieweit auf angebotsseitige Merkmale zurückzuführen sind. Bei den angebotsseitigen Merkmalen müsste zudem noch danach unterschieden werden, ob oder wie sie von Krankenkassen beeinflusst werden können. In diese Richtung ist auch das komplizierte Verfahren in den Niederlanden zu verstehen (vgl. oben 4.2), das in Deutschland aufgrund fehlender Daten gar nicht, oder erst nach sehr langen Vorlaufzeiten implementiert werden könnte. Aus diesem Grund wird als pragmatische Lösung eine einfache Heuristik vorgeschlagen: Regionale Unterschiede sollten im Risikostrukturausgleich nicht vollständig, sondern nur hälftig ausgeglichen werden. Sollten sich im Laufe der Zeit neue Erkenntnisse hinsichtlich der optimalen Quote ergeben, so ließe sich der Prozentsatz auch schnell anpassen.

51 Vgl. Wille, E./Schneider, U., Zur Regionalisierung in der gesetzlichen Krankenversicherung, Recht und Politik im Gesundheitswesen 2 (1997): 141-158.

52 BSGE 58, 135, 146 und oben 3.1; aber auch BVerfGE 113 S. 167 und oben 3.2. 


\subsection{Verfahrensbeschreibung}

Der morbiditätsorientierte Risikostrukturausgleich funktioniert zur Zeit so: Jede Krankenkasse erhält für jeden Versicherten eine Grundpauschale in Höhe der GKV-durchschnittlichen Ausgaben. Die Grundpauschale wird dann durch den Risikostrukturausgleich mit einem System von $\mathrm{Zu}$ - und Abschlägen an die konkrete Risikostruktur des Versicherten angepasst. Entsprechend dem Alter und Geschlecht (Alter-Geschlechts-Gruppen - AGG) gibt es in der Regel Abschläge, die die Höhe der Zuweisungen auf die durchschnittliche Höhe der Ausgaben eines gesunden Versicherten der entsprechenden Alters- und Geschlechtsgruppe reduziert. Für Erwerbsminderungsrentner (Erwerbsminderungsgruppen - EMG) und chronisch Kranke (Hierarchisierte Morbiditätsgruppen - HMG) gibt es dann Zuschläge auf diesen Basisbeitragsbedarf nach Alter und Geschlecht. Die Morbiditätszuschläge für chronisch Kranke erfolgen aufgrund der von den Krankenkassen gemeldeten Diagnosen und Verordnungen des Vorjahres. Die Höhe der Zuschläge richtet sich wiederum nach den durchschnittlichen mit der Krankheit verbundenen Folgekosten. Wie gezeigt wurde, ist dieses System nur bedingt in der Lage, regionale Ausgabenunterschiede auszugleichen.

Um diese regionalen Unterschiede besser zu berücksichtigen, wäre eine naheliegende Möglichkeit, neben den AGG, EMG und HMG zusätzlich 16 Ländergruppen (LG) zu bilden. Jeder Versicherte würde dann aufgrund seines Wohnsitzes der entsprechenden Ländergruppe für sein Bundesland zugeordnet. Diese LG würden dann neben die AGG, EMG und HMG in die Berechnung der $\mathrm{Zu}$ und Abschläge eingehen. So würde für jede Ländergruppe ein Länderzuschlag ermittelt, der die regionalen Ausgabenunterschiede erfasst. Dieser Länderzuschlag würde allerdings nur in halber Höhe ausgezahlt. Die andere Hälfte würde wieder in die $\mathrm{Zu}$ - und Abschläge der AGG, EMG und HMG zurückfließen.

Um diese (halbierten) Länderzuschläge für die Krankenkassen zahlungswirksam werden zu lassen, könnte eine Anpassung der Grundpauschalen erfolgen. Dazu müsste die Grundpauschale für Versicherte mit Wohnsitz in einem Land mit einem unterdurchschnittlichen Zuschlag reduziert und für einen Versicherten mit Wohnsitz in einem Land mit überdurchschnittlichem Zuschlag erhöht werden. Der Vorteil dieses Modells liegt auch darin, dass die hierfür notwendigen Daten bereits vollständig vorliegen, und es daher vom Bundesversicherungsamt ohne größeren zusätzlichen administrativen Aufwand schnell umsetzbar wäre. 


\subsection{Verteilungswirkung auf die Länderebene}

Um die Verteilungswirkung zu illustrieren, werden die Auswirkungen des Modells mit Daten aus dem Jahr 2008 illustriert. Die Ergebnisse werden in Tabelle 1 dargestellt. Zunächst wird der Landeszuschlag wiedergegeben. Er zeigt an, um wie viel Euro die Ausgaben eines Versicherten mit Wohnsitz in einem Bundesland hälftig höher sind als die Ausgaben eines Versicherten ohne Zuordnung zu einem Bundesland (insb. Versicherte mit Wohnsitz im Ausland). Der bundesdurchschnittliche jährliche Landeszuschlag beträgt 77,91 Euro. In Bundesländern mit höheren Landeszuschlägen sind also höhere Ausgabenniveaus nach Berücksichtigung der sonstigen im Risikostrukturausgleich eingebundenen Merkmale $\mathrm{zu}$ verzeichnen.

Tabelle 1: Finanzielle Eckdaten des Regionalmodells

\begin{tabular}{|c|c|c|c|c|}
\hline Wohnsitz (Bundesland) & $\begin{array}{c}\text { Landeszuschlag } \\
\text { (halbiert) }\end{array}$ & $\begin{array}{c}\text { Anpassung der } \\
\text { Grundpauschalen }\end{array}$ & $\begin{array}{c}\text { Umverteilung } \\
\text { (absolut) }\end{array}$ & $\begin{array}{c}\text { Umverteilung } \\
\text { (prozentual) }\end{array}$ \\
\hline Schleswig-Holstein & $89,77 €$ & $11,86 €$ & 24 Mio. $€$ & $0,5 \%$ \\
\hline Hamburg & $142,35 €$ & $64,44 €$ & 92 Mio. $€$ & $3,0 \%$ \\
\hline Niedersachsen & $72,54 €$ & $-5,37 €$ & -48 Mio. $€$ & $-0,3 \%$ \\
\hline Bremen & $95,63 €$ & $17,72 €$ & 9 Mio. $€$ & $0,8 \%$ \\
\hline Nordrhein-Westfalen & $69,83 €$ & $-8,08 €$ & -152 Mio. $€$ & $-0,5 \%$ \\
\hline Hessen & $83,17 €$ & $5,26 €$ & 17 Mio. $€$ & $0,2 \%$ \\
\hline Rheinland-Pfalz & $77,84 €$ & $-0,07 €$ & -7 Mio. $€$ & $-0,1 \%$ \\
\hline Baden-Württemberg & $90,30 €$ & $12,39 €$ & 95 Mio. $€$ & $0,5 \%$ \\
\hline Bayern & $93,85 €$ & $15,94 €$ & 143 Mio. $€$ & $0,7 \%$ \\
\hline Saarland & $84,82 €$ & $6,91 €$ & 4 Mio. $€$ & $0,2 \%$ \\
\hline Berlin & $101,40 €$ & $23,49 €$ & 58 Mio. $€$ & $0,9 \%$ \\
\hline Brandenburg & $65,91 €$ & $-12,00 €$ & -32 Mio. $€$ & $-0,6 \%$ \\
\hline Mecklenburg- & $68,79 €$ & $-9,12 €$ & -17 Mio. $€$ & $-0,5 \%$ \\
\hline Vorpommern & $39,38 €$ & $-38,53 €$ & -154 Mio. $€$ & $-1,8 \%$ \\
\hline Sachsen & $57,01 €$ & $-20,90 €$ & -51 Mio. $€$ & $-1,0 \%$ \\
\hline Sachsen-Anhalt & $37,83 €$ & $-40,08 €$ & -87 Mio. $€$ & $-1,9 \%$ \\
\hline Thüringen & & &
\end{tabular}

In der konkreten Anwendung würde der Abstand des ermittelten Landeszuschlages von dem bundesdurchschnittlichen Landeszuschlag zur Anpassung der Grundpauschale verwendet. Krankenkassen würden also für Versicherte mit Wohnsitz in Schleswig-Holstein eine um 11,86 Euro angehobene jährliche Grundpauschale erhalten, für Versicherte mit Wohnsitz in Niedersachsen eine um 5,37 Euro niedrigere jährliche Grundpauschale. Die bundesdurchschnittliche 
Grundpauschale würde so unverändert bleiben, und damit auch das Gesamtvolumen der Zuweisungen aus dem Gesundheitsfonds.

Die Änderungen der Zuweisungsvolumen sind in den letzten beiden Spalten enthalten. Beispielsweise erhalten die Stadtstaaten so höhere Zuweisungen (Hamburg + 92 Mio. Euro, Bremen +9 Mio. Euro, Berlin +58 Mio. Euro). Auch wenn die Entlastungen und vor allem Belastungen als absolute Millionenbeträge sehr hoch aussehen, müssen diese natürlich vor dem Hintergrund des Volumens der Gesamtzuweisungen gesehen werden. So bedeutet der Rückgang der Zuweisungen für Versicherte mit Wohnsitz in Nordrhein-Westfalen von 152 Millionen Euro im Verhältnis zu den Gesamtzuweisungen lediglich einen Rückgang von $0,5 \%$.

Abbildung 4: Über- und Unterdeckungen je Bundesland vor und nach Einführung

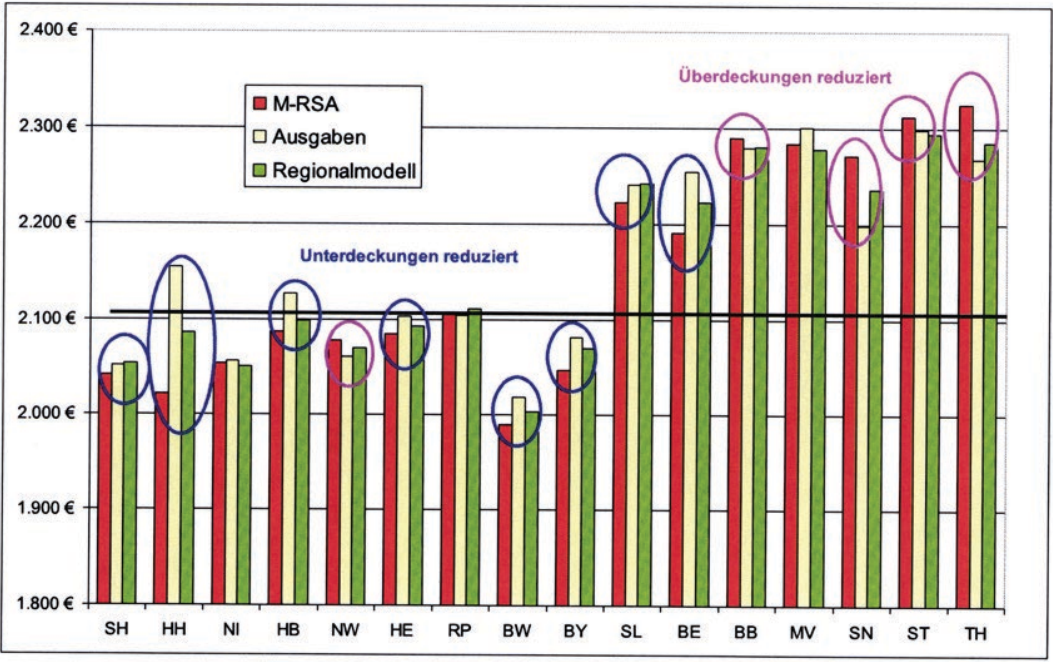

Kann damit das Ziel erreicht werden, regionale Über- und Unterdeckungen zu reduzieren? Dafür werden in Abbildung 4 die Zuweisungen im morbiditätsorientierten Risikostrukturausgleich ohne Regionalkomponente (rote Säulen) und die Zuweisungen mit Regionalkomponente (grüne Säulen) den Ausgaben (gelbe Säulen) gegenübergestellt. Es zeigt sich, dass in fast allen Bundesländern die bestehenden Über- oder Unterdeckungen reduziert werden. Beispielswiese be- 
tragen die durchschnittlichen Ausgaben je Versicherten in Hamburg 2.154 Euro, während die durchschnittlichen Zuweisungen im morbiditätsorientierten Risikostrukturausgleich nur 2.021 Euro betrugen. Mit dem Regionalmodell werden die Zuweisungen auf 2.086 Euro erhöht. Damit wird die Überdeckung abgebaut, ohne dass der Druck zur Kostenreduzierung ganz aufgehoben wird. 
Eberhard Wille and Klaus Knabner - 978-3-631-75550-1 Downloaded from PubFactory at 01/11/2019 03:39:43AM via free access 
Notwendigkeiten und Chancen einer dezentralen Gesundheitsversorgung für eine landesweit kalkulierende Krankenkasse

\section{Rolf Hoberg}

\section{Ausgangslage: Zunehmende Zentralisierung}

Die Gesetzgebung der letzten Jahre hat auf der Finanzierung-, Vergütungs- und Steuerungsseite einen massiven Zentralisierungsschub ausgelöst. Durch den Gesundheitsfonds und den Morbi-RSA erfolgt eine zentralisierte, bundeseinheitliche Bereitstellung von Finanzmitteln für die Krankenkassen.

Die Zentralisierung auf der Finanzierungsseite erfordert, wenn es nicht zu regionalen Verwerfungen kommen soll, eine gleichgerichtete Zentralisierung der Vergütungssysteme und auch der Steuerungsansätze.

Konsequenterweise erfolgt diese auch im Bereich der ärztlichen Vergütung mit Punktwert- und Honorarangleichung (mit unbeabsichtigten Nebenfolgen neuer Verwerfungen, die politisch auffällig werden) und auch bei den Vergütungen im stationären Bereich mit einer Konvergenz der Landesbasisfallwerte. Durch den Gemeinsamen Bundesausschuss werden Leistungskatalog und zunehmend auch Details der Leistungsgewährung bundeseinheitlich geregelt.

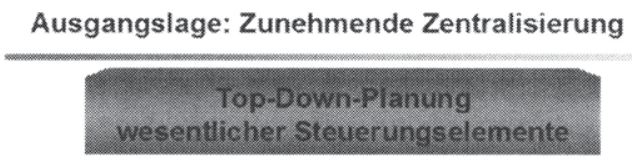

Bundesebene

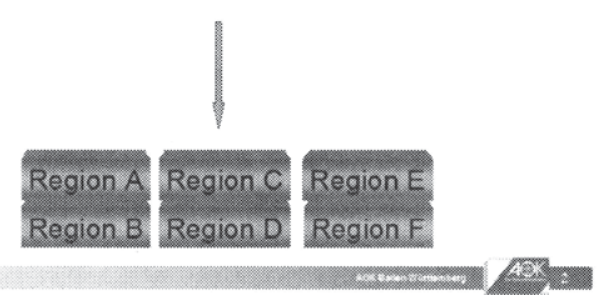

Die Wirklichkeit der Gesundheitsversorgung in der Bundesrepublik Deutschland weist aber sowohl von den Versorgungsbedarfen als auch von den regionstypischen Mustern unterschiedlich gestalteter, möglicherweise aber gleich effi- 
zienter Versorgungskonstellationen, erhebliche Unterschiede auf, die sich auch durch intensives Bemühen um immer ausgefeilterer Vergütungs- und Steuerungssysteme nicht vollständig und richtig abbilden lassen.

Ausgangslage: Zunehmende Zentralisierung

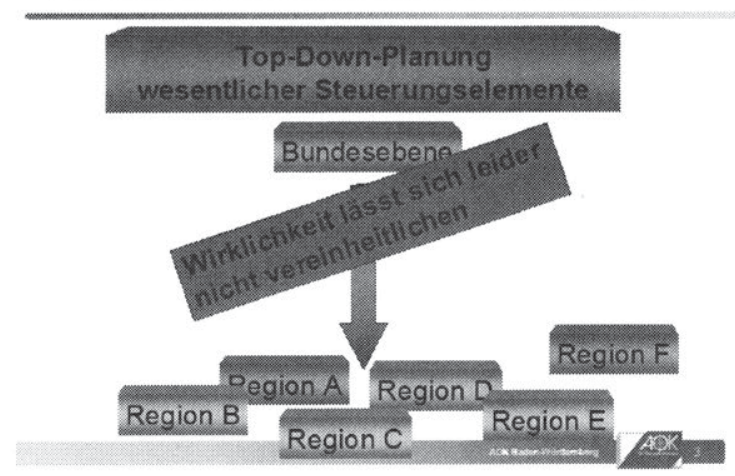

Vergleicht man zwischen den Bundesländern z. B. die soziodemografische Grundsituation, so stellt man sowohl bei dem Anteil der über 65-Jährigen als auch der unter 18-Jährigen erhebliche regionale Unterschiede fest.

\section{Anteil über 65-Jähriger in Prozent}

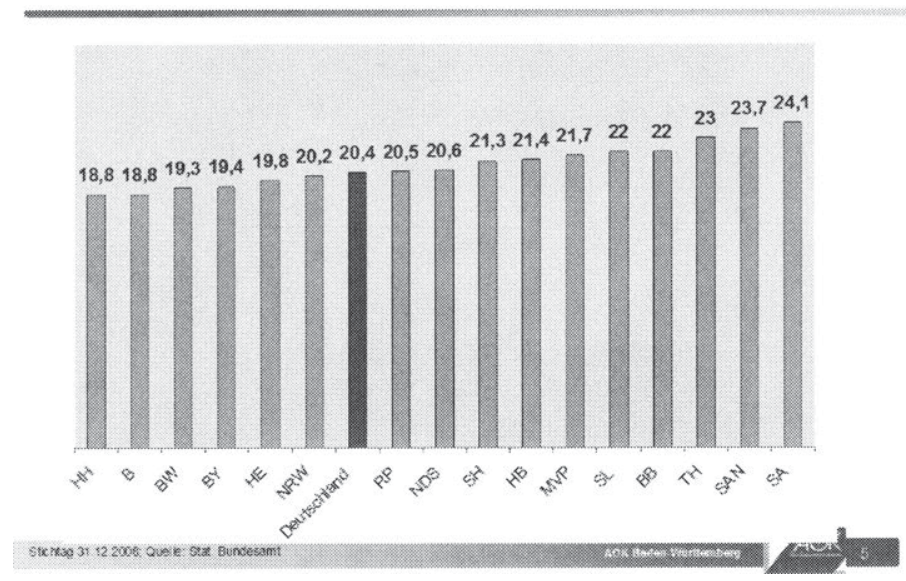




\section{Anteil unter 18-Jähriger in Prozent}

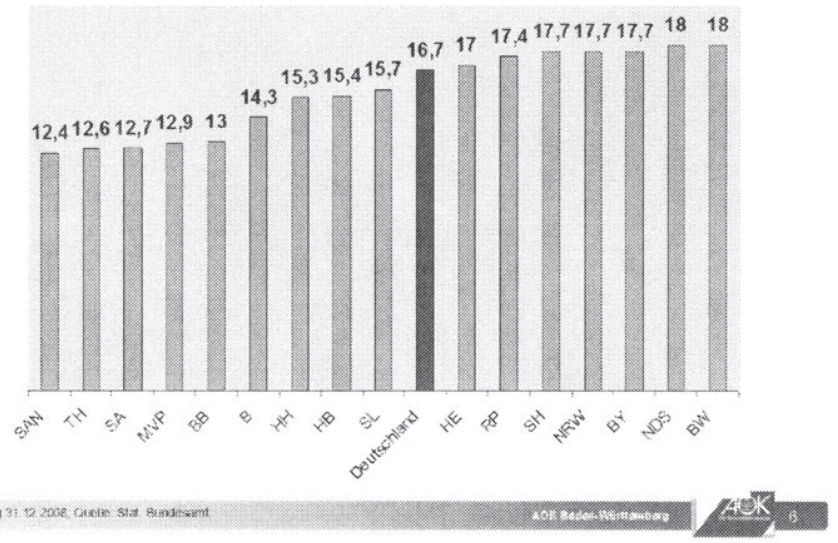

Auch die Bevölkerungsdichte, die ja ein indirekter Ausdruck für die Erreichbarkeit von Versorgungsangeboten ist, weist zwischen den Bundesländern eine erhebliche Spreizung auf.

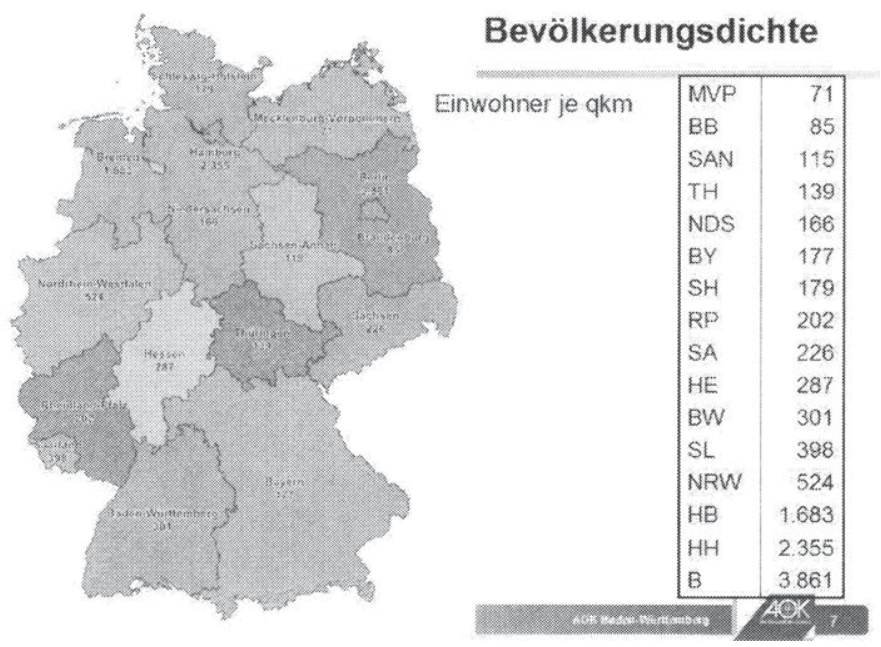

Betrachtet man dann die unterschiedlichen Versorgungsstrukturen im stationären, ambulanten Bereich und die Bereitstellung anderer Leistungen, so zeigen 
sich auch hier erhebliche Unterschiede zwischen den Bundesländern. So variiert z. B. die Bettendichte zwischen den Flächenstaaten Baden-Württemberg mit 55,1 Betten je 10.000 Einwohnern und Thüringen mit 70 Betten je 10.000 Einwohnern. Die Arztdichte weist ebenfalls erhebliche Unterschiede zwischen den Bundesländern auf,

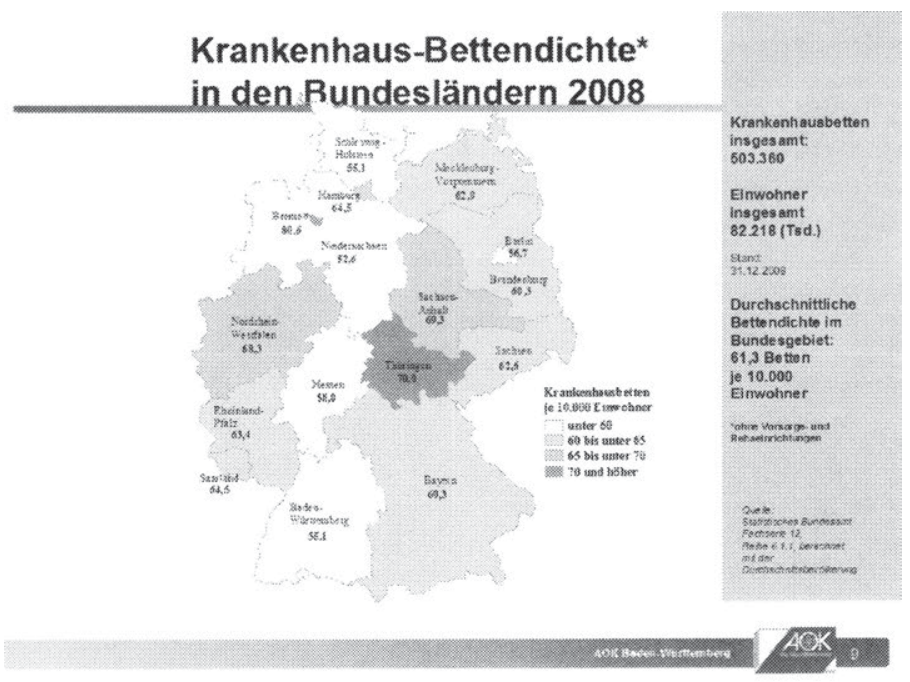

Arztdichte in den

Bundesländern 2008

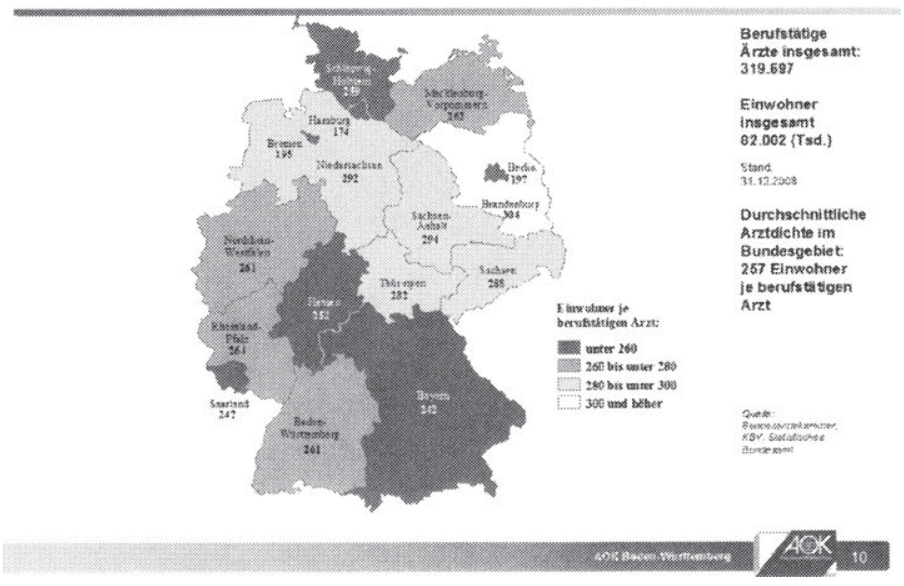


die bei einem komplementären Muster auf die unterschiedliche Gewichtung der Versorgung durch den ambulanten und stationären Bereich hindeuten.

Bei den veranlassten Leistungen zeigt sich am Beispiel der über die AOK abgerechneten Leistungen ein deutliches, nicht aus den Bedarfsanforderungen aus der Altersstruktur der Bundesländer erklärbares Muster. Vergleicht man die Arzneimittelausgaben, so sieht man in der Gesetzlichen Krankenversicherung einen Ost-West-Unterschied von über 10 Prozent.

\section{Gesundheitswesen als komplexes System}

Das Gesundheitswesen der Bundesrepublik Deutschland zeichnet sich durch eine außerordentliche Vielschichtigkeit mit unterschiedlichsten Akteuren, die jeweils eigene Interessen mit verschiedenen Instrumenten verfolgen, aus.

Diese Komplexität erfordert entweder entsprechend hoch integrierte komplexe Steuerungssysteme oder - was der sinnvollere Weg ist - eine Reduzierung der Komplexität durch konsequente Sektoralisierung, mit der allerdings in erheblichem Umfang Synergien verschenkt werden bzw. eine konsequente Regionalisierung und Dezentralisierung. Hier wird die Auffassung vertreten und mit Praxisbeispielen gestützt, dass konsequente Dezentralisierung zu einem effektiveren Gesundheitswesen führt.

\section{Gesundheitswesen als komplexes System}

\section{Vielzahl an Akteuren mit unterschiedlichen}

Zielen und Interessen

$\rightarrow$ Notwendigkeit, Komplexität zu reduzieren

Entweder konsequente Sektoralisierung oder

Konsequente Regionalisierung/Dezentralisierung 
Notwendigkeit: bedarfsorientierte Steuerung

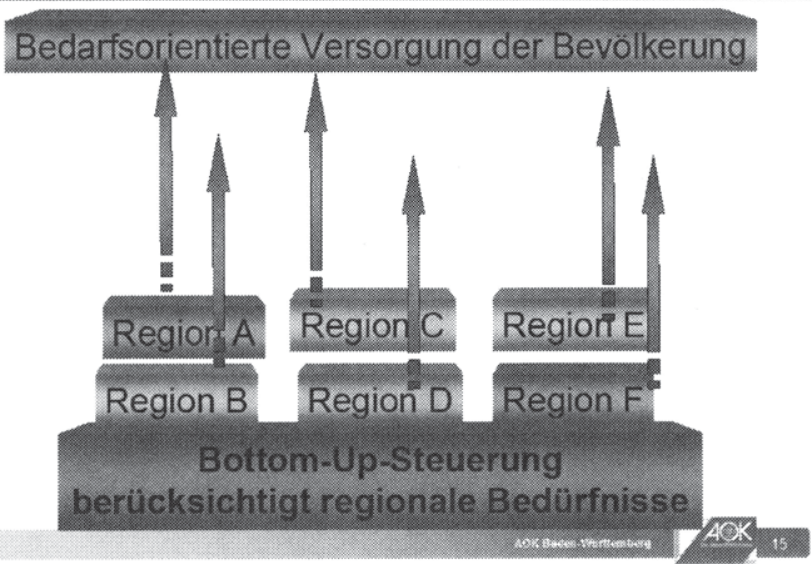

\section{Regionale Versorgungsplanung als Chance}

Eine Versorgungsplanung auf der Ebene der Bundesländer erscheint als eine Möglichkeit, Komplexität auf ein gerade noch beherrschbares Maß zu reduzieren (dabei muss allerdings gesehen werden, dass im Vergleich der Länder in der $\mathrm{EU}$, manche der Bundesländer größer sind als manche in der EU vertretenen Nationalstaaten).

Regionale Versorgungsplanung ermöglicht eine genauere Feststellung von regionalen Bedarfen; vermeidet, durch eine bessere Kenntnis des Versorgungsbedarfes und der angebotenen Kapazitäten, die sonst unvermeidliche Über-, Unter- und Fehlversorgung. Durch eine teilräumliche Gliederung ist es möglich, sektorübergreifende Kooperationen wirksam zwischen Leistungserbringern im Hinblick auf vorhandene Patientenströme zu organisieren und schließlich gelingt es so, auch kleinere Organisationsgruppen effektiv und mit Verbindlichkeit in die Planung und Versorgung einzubeziehen.

Daneben ist auch die Verbesserung der Qualitätssicherung in überschaubaren Gebieten, die von Patienten auch als erlebter Versorgungsraum in einen effektiven Qualitätsvergleich einbezogen werden, machbar. 
Gleichzeitig wird man damit auch dem Verfassungsauftrag, dass Gesundheitsversorgung Länderaufgabe ist, wieder gerecht werden können und findet so Ansatzpunkte für eine politische Legitimation eines ansonsten bundeszentral durch Akteure mit sinkender Glaubwürdigkeit gesteuerten Gesundheitssystems.

\title{
Regionale Versorgungsplanung als Chance
}

\author{
Bedarfsgerechte Versorgung \\ Vermeidung von Über-, Unter- und \\ Fehlversorgung \\ Möglichkeiten zu sektorenübergreifenden \\ Kooperationen \\ Einflussmöglichkeiten kleinerer \\ Organisationsgruppen steigen \\ Kooperationen mit der Landesregierung \\ Qualitätssicherung vor Ort (z. B. Qualitätszirkel)
}

\section{Regionale Versorgung am Beispiel einer landesbezogen kalkulierenden Krankenkasse}

\subsection{Prävention}

Bereits heute bieten sich in einigen Feldern regionale Handlungsmöglichkeiten, die genutzt werden können, um den Zentralisierungstendenzen des Kollektivvertragsbereiches zu entkommen.

Am Beispiel der AOK Baden-Württemberg kann gezeigt werden, wie durch intensive Kooperation und flächendeckende Angebote im Bereich der Prävention nachhaltig die Gesundheit eines großen Bevölkerungsteils verbessert werden kann. Die AOK Baden-Württemberg versichert von den rd. 11 Mio. Einwohnern des Landes Baden-Württemberg 3,8 Mio. Beispiele für flächendeckende Angebote im Bereich der Prävention durch die AOK Baden-Württemberg finden sich in Abbildung 17. Für einen im Durchschnitt besseren Gesundheitszustand der baden-württembergischen Bevölkerung liegen eine Reihe von Indikationen vor 
(Lebenserwartung, Prävalenz chronischer Erkrankungen, Body-Mass-Index, etc.).

\section{Beispiel: Prävention}

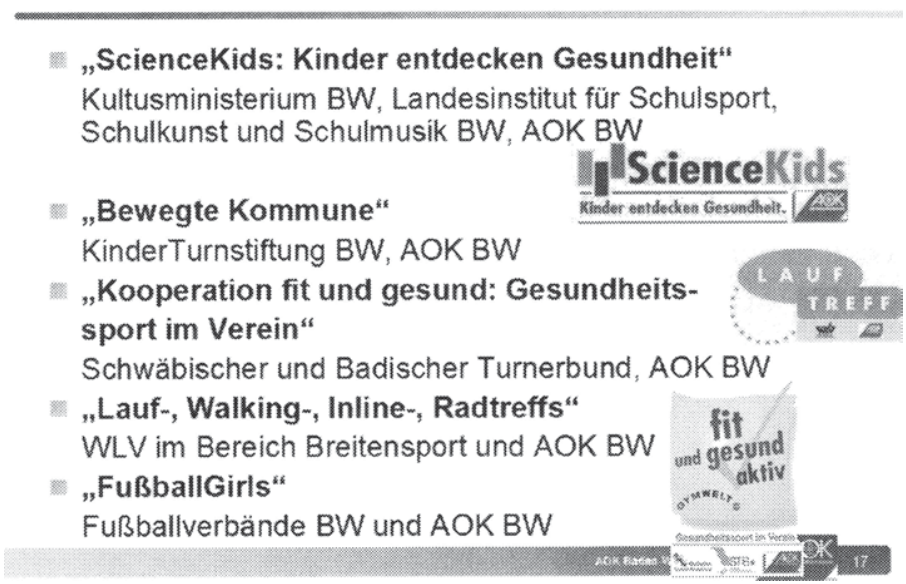

\subsection{Selektivverträge nach $\S 73$ b und $\S 73$ c SGB V}

Einen weiteren Ansatz für regionale Versorgungsgestaltung bietet die Nutzung der Optionen aus $\S 73$ b und $\S 73$ c SGB V. Mit der Option, aus dem kollektivvertraglichen System auszusteigen und mit relevanten Arztgruppen zu eigenen Versorgungsverabredungen zu kommen, gelingt es im Interesse der Versicherten in Baden-Württemberg eine den hiesigen Versorgungsbedürfnissen angepasste Versorgung zu organisieren. Dieses gelingt sowohl auf der Vergütungsseite als auch auf der Seite der Versorgungssteuerung mit beachtlichem Erfolg.

Die Ziele der Hausarztzentrierten Versorgung sind:

- die Sicherung einer flächendeckenden ambulanten hausärztlichen Versorgung.

- die Erhaltung regionaler Handlungsautonomie sowohl für Gruppierungen der Ärzteschaft als auch für eine regional agierende Krankenkasse.

- der Abbau von Bürokratieerfordernissen aus der Vergütungs- und Prüfsystematik im Bereich der Kollektivverträge. 
- selbstverständlich auch die Möglichkeit zur wettbewerblichen Differenzierung sowohl für die Krankenversicherung als auch für die Ärzte und damit die Eröffnung von Wahlalternativen für die Versicherten.

Der Ansatz zu einer verbesserten Vergütung für die Ärzte gelingt natürlich nur, wenn mittelfristig Effizienzpotentiale gehoben werden und durch eine innovative Versorgungssteuerung sowohl die Qualitätsorientierung als auch die Populationsorientierung der Versorgung gewährleistet werden kann.

Dass dieses Programm im Bereich der Hausarztzentrierten Versorgung ein Erfolg ist, zeigt ein Blick auf die Einschreibezahlen und die Landkarte. Der Hausarztvertrag, der seit 01.07.2008 in Kraft ist, umfasst inzwischen ca. 3.700 Hausärzte, die sich eingeschrieben haben und annähernd 1 Mio. teilnehmende Versicherte, die von diesem Angebot Gebrauch machen.

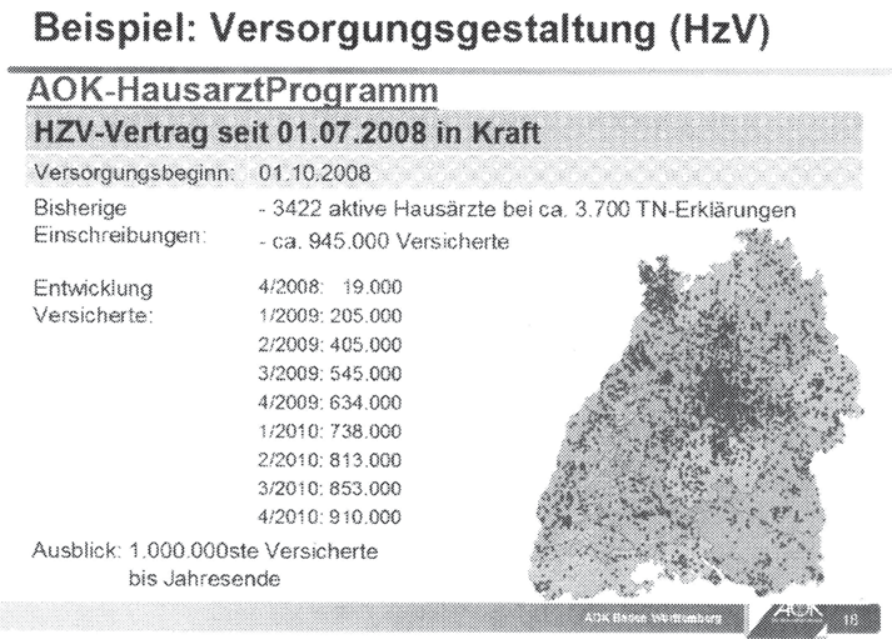

Für eine populationsorientierte Gesamtversorgung ist es erforderlich, dass neben den Grundversorgungsfunktionen des Hausarztes auch alle Möglichkeiten genutzt werden, um die Lotsenfunktion des Hausarztes wirksam werden zu lassen. Dazu schließt die AOK Baden-Württemberg schrittweise eine Reihe von $\S 73 \mathrm{c}$ Verträgen für die entsprechende fachärztliche Versorgung. Hausarztzentrierte Versorgung und facharztzentrierte Versorgung nehmen so aufeinander Bezug und schöpfen weitere Effizienzpotentiale. In Baden-Württemberg liegen mitt- 
lerweile vor: ein ergänzender Facharztvertrag für den Bereich Kardiologie, für den Bereich der Gastroenterologie. Derzeit in Verhandlung befindet sich ein Vertrag für Psychiatrie, Neurologie, Psychotherapie. Weitere Verträge werden folgen.

\section{Verknüpfung HZV mit 73c-Verträgen}

\section{HZV und Facharztverträge spielen zusammen und ergänzen sich}

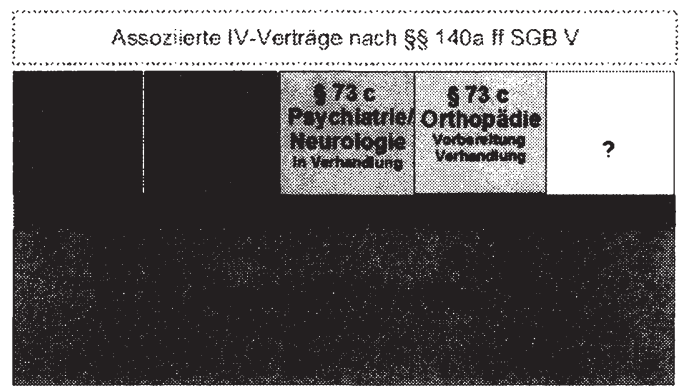

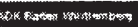

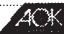

Durch die Verknüpfung von Hausarztzentrierter Versorgung und Facharztverträgen erschließen sich für die Versicherten eine Vielzahl von Vorteilen. Dieses beginnt bei Servicevorteilen in der Terminvergabe bei Fachärzten, mit Abendsprechstunden und garantiert limitierten Wartezeiten. Für die Versicherten fallen bei rabattierten Medikamenten die Zuzahlungen fort und die Präventionsanstrengungen durch koordinierte Betreuung seitens des Hausarztes in Verknüpfung mit den entsprechenden Fachärzten werden deutlich verbessert. Insbesondere dadurch, dass zwischen Haus- und Fachärzten abgestimmte Versorgungspfade, Schnittstellenabsprachen und Kommunikationsmedien festgelegt worden sind, steigt die Effizienz der Versorgung.

Daraus ergibt sich eine vielfältige Vorteilssituation für Ärzte, Versicherte und die AOK Baden-Württemberg. 


\section{Regionalisierung der Einnahmeseite}

Die Bemühungen um eine Regionalisierung der Versorgung finden allerdings immer noch im Rahmen bundeseinheitlicher Finanzierungszuweisungen statt. Betrachtet man die für Baden-Württemberg unterdurchschnittlichen Leistungsausgaben, bei zugleich unterdurchschnittlich niedrigen Zuweisungen aus dem Gesundheitsfonds und stellt dem die relativ hohe Erwirtschaftung von Beiträgen im Lande gegenüber, so wird deutlich, dass im Vergleich zu anderen Regionen durchaus Spielräume für eine Verbesserung des Einnahmerahmens mit mehr an regionaler Gestaltungsgerechtigkeit gegeben sind (vgl. Beitrag v. Dr. Gaßner i. d. Band).

\section{Fazit}

Die Komplexität des deutschen Gesundheitssystems lässt sich am besten durch Dezentralität bewältigen. Hierzu sollten sowohl auf der Vertragsseite, wie auch auf der Seite von Vergütungen aber auch der Einnahmenseite neue Spielräume geschaffen werden. 
Eberhard Wille and Klaus Knabner - 978-3-631-75550-1 Downloaded from PubFactory at 01/11/2019 03:39:43AM via free access 


\section{Möglichkeiten einer dezentralen Geschäftspolitik aus Sicht einer bundes- weit kalkulierenden Krankenkasse}

\section{Norbert Klusen}

Sich wandelnde Rahmenbedingungen und die aktuelle gesundheitspolitischen Diskussion um Regionalisierung stellen auch eine bundesweit aufgestellte Krankenkasse vor die Frage einer verstärkten Verlagerung von Aktivitäten in die Regionen. Als eine von 84 bundesweit agierenden Krankenkassen ${ }^{1}$ versichert die Techniker Krankenkasse (TK) mit 7,7 Millionen Versicherten rund 10\% der GKV-Versicherten aus dem gesamten Bundesgebiet. Während die TK mit einem bundesweiten Marktanteil von 10,7\% an zweiter Stelle in der gesetzlichen Krankenversicherung liegt, variieren die regionalen Marktanteile in den Bundesländern teilweise erheblich. Welche Notwendigkeiten, vor allem aber auch welche Möglichkeiten für eine Dezentralisierung bestehen unter den aktuellen Bedingungen aus Sicht der TK?

In der Logik einer bundesweit agierenden Kasse basiert ein Großteil des unternehmerischen Erfolgs der TK in erster Linie auf einer zentral gesteuerten Geschäftspolitik. Alle relevanten strategischen und finanziellen Entscheidungen werden auf zentraler Ebene getroffen, um eine bundesweit einheitliche Unternehmenspolitik auf der Basis der zentralen unternehmerischen Zielstellungen der TK sicherzustellen. Die Umsetzung der zentralen Vorgaben erfolgt durch ein Netz von Unternehmenseinheiten mit unterschiedlichem Dezentralisierungsgrad, wie Geschäftsstellen zur Kundenberatung, Servicezentren und Vertriebszentren. Das Spannungsfeld zwischen den Unternehmenszielen einer kundennahen Versorgung einerseits und dem Wirtschaftlichkeitsgebot andererseits erfordert bei den Kundenservices das Austarieren einer flexiblen Balance zwischen dezentralen und zentralen Unternehmensstrukturen: Neben einem flächigen Netz dezentraler Geschäftsstellen wird eine Reihe von Kundenservices zentral angeboten, wie eine umfassende und interaktive Internetplattform, eine differenzierte zentrale Telefonie und eine große Bandbreite fachlicher Hotlines, die von den Versicherten ausgesprochen gut angenommen werden. So gehen bei einem Gesamtvolumen von ca. 91 Millionen Kundenkontakten jährlich rund 15 Millionen Kundenanrufe in den Telefoniezentren und Kundenberatungen ein, bei gleichzeitig 2,1 Millionen Besuchen in den Kundenberatungen. Gerade die Möglich-

${ }^{1}$ ohne Landwirtschaftliche Krankenkassen und Knappschaft 
keit, Angebotsstrukturen dort wo zielführend zentral anzubieten, gehört zu den großen Vorteilen einer bundesweiten Kasse. Im Interesse der Kunden an einem wirtschaftlichen Umgang mit den Mitgliederbeiträgen führen kontinuierliche interne Struktur- und Prozessoptimierungen immer wieder zur Bündelung von Know-How und Kompetenzen in zentralen Einheiten.

Gleichzeitig wird die zentral gesteuerte Geschäftspolitik der TK schon heute ergänzt um gelebte Regionalität. Im Zuge einer bewussten unternehmenspolitischen Entscheidung verfolgt die TK bereits seit Jahren den Aufbau eines regionalen Profils. Die Präsenz der TK in den Ländern wird in erster Linie durch 15 Landesvertretungen erreicht, deren Hauptaufgaben darin bestehen, im Rahmen der zentralen Allokations- und Durchführungsentscheidungen das Vertragsgeschäft vor Ort zu gestalten und Konzepte für Versorgungsprodukte zu entwickeln und umzusetzen. Dabei dienen sie als Seismographen für die Besonderheiten der örtlichen Versorgungslandschaften, Bedarfe, Strukturen und Wettbewerbssituationen und gestalten durch politische und öffentliche Präsenz in den Ländern die Rahmenbedingungen der Versorgung im Sinne der Versicherten vor Ort mit. Auf eigenen Internetseiten, die zur Wahrung der Gesamtmarke in das einheitliche Design der TK eingepasst sind, kommunizieren die einzelnen Landesvertretungen ihr Profil und informieren über ihre Angebote und Aktivitäten. Darüber hinaus besitzt die TK mit dem Newsletter "TK Spezial" ein regionales Informationsmedium, das durch die einzelnen Landesvertretungen herausgegeben wird und der Information der Vertragspartner vor Ort dient. Eine mittlerweile fast historische Besonderheit gelebter Regionalität der TK sind die rund 11.000 ehrenamtlichen Mitarbeiter, deren wohnort- und arbeitsplatznahe Beratung von TK-Kunden eine gewachsene und bewährte Tradition darstellt. Alle regionalen Aktivitäten sind jedoch immer in den Rahmen der einheitlichen Steuerung durch die Zentrale eingebettet. Auf diese Weise erreicht die TK schon heute eine klare Stärkung ihrer Marke in den Ländern und die regionale Nähe zum Kunden, während sie gleichzeitig bundesweit kongruentes Handeln durch eine übergreifende Strategie und ein gemeinsames Erscheinungsbild sicherstellt.

Mit dem Koalitionsvertrag der aktuellen Bundesregierung wurde durch die Ankündigung der Überführung des finanziellen Ausgleichssystems "in eine Ordnung mit mehr Beitragsautonomie, regionalen Differenzierungsmöglichkeiten und einkommensunabhängigen Arbeitnehmerbeiträgen" dem politischen Willen nach verstärkter Regionalität Ausdruck gegeben. Die politische Debatte um deren mögliche Ausgestaltung ist seitdem im Gange und kommt in verschie- 
denen Gutachten zum Ausdruck, die die Forderung nach mehr Regionalisierung vorrangig in Bezug auf die Kasseneinnahmen diskutieren. Im Licht der politischen Diskussion sind aus Sicht der TK die Möglichkeiten eines weiteren Ausbaus dezentraler Strukturen insbesondere unter den Aspekten der Versorgungsnotwendigkeiten, Wirtschaftlichkeitserwägungen und Wettbewerbsbedingungen einer bundesweiten Krankenkasse abzuwägen.

\section{Mehr Regionalisierung als Chance zur Versorgungsoptimierung?}

Die Anforderungen an eine bedarfsgerechte Versorgung und die Versorgungsinfrastruktur befinden sich im Wandel und stellen die regionale Versorgungsgestaltung zunehmend vor neue Herausforderungen. Neben sich verändernden gesellschaftlichen Strukturen, die vor allem durch die demographische Alterung bedingt sind und neue Versorgungsbedarfe mit sich bringen, führt auch eine zunehmende ärztliche Ungleichverteilung mittelfristig zu Handlungsbedarf. Die schon heute in wenigen Regionen bestehende Unterversorgung mit Ärzten wird sich angesichts der abnehmenden Bereitschaft von Medizinstudenten, in ländliche Gegenden zu gehen, weiter verschärfen. Schon heute weisen zunehmend mehr Patienten die Bereitschaft auf, für eine hochwertige medizinische Behandlung längere Wege in Kauf zu nehmen. Vor diesem Hintergrund plädiert der Sachverständigenrat im Gesundheitswesen in seinem Gutachten 2009 für eine "koordinierte, populationsbezogene, regional differenzierte Versorgung". ${ }^{2}$ Dabei wird die künftige Herausforderung wird zum einen darin bestehen, eine qualitativ hochwertigen flächendeckenden Versorgung besonders in dünn besiedelten strukturschwachen Gebieten sicherzustellen. Neben der Dezentralisierung von Leistungsangeboten, auch durch einen größeren Anteil mobiler Leistungen, bedeutet das aber zum anderen auch die Zentralisierung spezieller Versorgungsangebote durch die Konzentration von medizinischer Infrastruktur an zentralen Orten.

Für eine kundengerechte Ausgestaltung dezentraler Versorgungsangeboten müssen vor allem die Wünsche der Patienten maßgeblich sein. Hierzu führt die TK regelmäßig repräsentative Umfragen durch, um die Wünsche und Bedarfe der Versicherten zu ermitteln. Die seit Jahren hohen Patientenpräferenzen für eine koordinierte interdisziplinäre Zusammenarbeit (69\% der Befragten geben dies als "sehr wichtig" im Krankheitsfall an), einen schneller Zugang zu medizi-

2 Sachverständigenrat zur Begutachtung der Entwicklung im Gesundheitswesen, Sondergutachten 2009 
nischen Koriphäen (65\%), kurze Wartezeiten und schnelle OP-Termine (65\%) bei einer hohen Versorgungsqualität (64\%) geben hinsichtlich der Regionalität von Versorgungsangeboten ein differenziertes Bild. ${ }^{3}$ Während der Wunsch nach koordinierten Behandlungspfaden vor allem die Notwendigkeit wohnortnaher Versorgungsangebote bedeutet, kann ein schneller Zugang zu Fachspezialisten auch über weitere Entfernungen organisiert werden.

Nicht zuletzt stellt eine regional differenzierte Morbiditätsstruktur Herausforderungen an eine regional angepasste Versorgung. So zeigt der jährliche Gesundheitsreport der TK 2006 starke regionale Unterschiede in der Prävalenz psychischer Erkrankungen: Gemessen an Fehlzeiten aufgrund Arbeitsunfähigkeit lagen Berlin und Hamburg mit ca. 30\% deutlich über dem Bundesdurchschnitt, und auch bei der Auswertung der ambulanten Diagnosen ist Berlin mit $24 \%$ über Durchschnitt klarer Spitzenreiter. ${ }^{4}$

Auswertungen wie diese zeigen, dass regional verschieden gearteter und unterschiedlich hoher Handlungsbedarf besteht, dem durch die Kollektivverträge der Krankenkassen nur bedingt entsprochen werden kann. Hier bieten Selektivverträge grundsätzlich eine gute Möglichkeit, Versorgung vor Ort kundennah und bedarfsorientiert zu gestalten. Im Gegensatz zum Kollektivvertrag ermöglichen sie eine flexible und zeitnahe Umsetzung regionaler Versorgung. Die TK setzt das Instrument regionaler Selektivverträge seit einigen Jahren erfolgreich ein mit den Zielen einer verbesserten interdisziplinären und intersektoralen Koordination der Versorgung, der Sicherstellung hoher Qualität und innovativer Versorgung, einem schnellen Zugang zu qualitativ hochwertiger Versorgung sowie Wirtschaftlichkeit der Angebote. Auch Selektivverträge stehen aber im Spannungsverhältnis zwischen wohnortnaher Patientenorientierung und Wirtschaftlichkeit und damit vor der Herausforderung einer angemessenen Balance zwischen dezentralen und zentralen Versorgungsstrukturen. Regionale Einzellösungen bieten zwar die Möglichkeit, auf regionalspezifische Bedarfe und Strukturen vor Ort aufzubauen, gehen aber häufig mit einem hohen Verwaltungsaufwand und hohen Transaktionskosten einher. Mehr als eine Vielzahl regionalspezifischer Einzelverträge, ist das langfristige Ziel aus Sicht einer bundesweiten Kasse ein überregionales Angebot von Selektivverträgen, die durch die Ausdehnung regional entwickelter Modelle auf andere Regionen mit vergleichbaren

\footnotetext{
${ }^{3}$ TK Trendmonitor 2009

${ }^{4}$ TK Gesundheitsreport 2008; jährliche Analyse der Fehlzeiten und ambulanten Diagnosedaten erwerbstätiger TK-Mitglieder
} 
Strukturen sowie dank eines zentralen Controllings Transaktions- und Verwaltungskosten senken und dadurch langfristig Wirtschaftlichkeitseffekte erzielen. Darüber hinaus werden spezialmedizinische und hochpreisige Leistungen in Form von Selektivverträgen bewusst überregional und zentral angeboten. Hier ist aus Gründen der Wirtschaftlichkeit wie auch der Qualitätssicherung im Interesse des Versicherten ein Angebot durch wenige zentrale Einheiten mit einheitlichen Strukturen geboten, wie dies die TK beispielsweise mit ambulanten Zentren zur PET-CT Diagnostik umsetzt.

Ein Beispiel für ein regionales Versorgungskonzept, das derzeit als regionaler Nukleus erprobt wird mit der Option einer späteren überregionalen Ausdehnung, ist das TK-Ärztenetz in Mecklenburg-Vorpommern. Durch die strukturierte Vernetzung von Ärzten unterschiedlicher Fachrichtungen, einem systematischen Überweisungsmanagement und garantierten maximalen Wartezeiten haben die Versicherten auch in unterversorgten Gebieten die Möglichkeit eines schnellen Zugangs zur Versorgung. Dabei wird eine hohe Versorgungsqualität durch definierte Qualitätskriterien, wie eine regelmäßige ärztliche Teilnahme an Qualitätszirkeln sowie eine leitliniengerechte Behandlung, sichergestellt. Effizienzmaßnahmen wie die Umsteuerung auf rabattierte Arzneimittel unterstützen die Wirtschaftlichkeit des Modells. Derzeit wird das Konzept zunächst innerhalb Mecklenburg-Vorpommerns ausgebaut. Für eine weitergehende Ausdehnung auf andere Regionen wird ausschlaggebend sein, inwieweit in dem Projekt Effektivitäts- und Effizienzziele im Sinne der Versicherten durch nachhaltige Anreizsysteme für die Leistungserbringer erreicht werden, sowie nicht zuletzt von der medizinischen Infrastruktur in anderen Regionen. Die Professionalität und Motivation der kooperierenden Partner ist eine wesentliche Voraussetzung für den Erfolg regionaler Angebote.

Ein erfolgreiches dezentrales Versorgungskonzept der TK ist auch das Netzwerk psychische Gesundheit. Für die TK besteht durch einen überproportional hohen Anteil Versicherter mit psychischen Diagnosen hier besonderer Handlungsbedarf. Nicht zufällig startete das Modell Ende 2009 zunächst in der überdurchschnittlich betroffenen Region Berlin. Als ein explizit regional ausgerichtetes Versorgungsmodell vereint der gemeindepsychiatrische Ansatz des Projekts sowohl gesundheitliche als soziale kommunale Leistungsanbieter mit dem Ziel, psychisch Kranken statt der häufig nicht erforderlichen Krankenhausbehandlung eine ambulante Therapie vor Ort zu ermöglichen. Eine wichtige Steuerungskomponente ist hierbei ein erfolgsabhängiger Vergütungsanteil im Rahmen 
übertragener Budgetverantwortung. Der regionale Ansatz zeigt sich schon jetzt als außerordentlich erfolgreich und wurde bereits auf Hamburg, Bremen und Kiel ausgeweitet mit dem Ziel einer weiteren Ausdehnung. Für das Ziel des Vertrags, eine strukturelle Umstellung von der stationären auf eine ambulante Versorgung, zu erreichen, stößt die TK als bundesweite Kasse mit begrenzten regionalen Marktanteilen an ihre Grenzen. Denkbar wäre hier eine Kooperation mit anderen Kostenträgern zur Sicherung ausreichender Marktanteile zur Umsetzung struktureller Änderungen und für die nachhaltige Wirtschaftlichkeit regionaler selektiver Versorgung.

\section{Mehr Regionalisierung vor dem Hintergrund des Wirtschaftlichkeits- gebots?}

Seit der Einführung des Gesundheitsfonds und der Zuweisungen des MorbiRSA mit einem erhöhten Umverteilungsvolumen hat die Bedeutung der Regionen für die einzelne Kasse zugenommen. Ein nachhaltiges Wirtschaften und das Erzielen positiver Gesamtdeckungsbeiträge stellt bundesweite Kassen zu Zeiten des Gesundheitsfonds vor die Herausforderung der genauen Kenntnis und Steuerung regionaler Leistungsausgaben.

Eine Regionalisierung der Kasseneinnahmen dagegen ist aus Sicht der TK kein zukunftsweisendes Konzept und aus Wirtschaftlichkeitserwägungen nicht sachdienlich. Die häufig geforderte regionale Anpassung der Einnahmen an die nach wie vor unterschiedlichen regionalen Vergütungsniveaus kann kein Ziel sein, denn die Preisunterschiede bestehen weitestgehend ungerechtfertigt und sind Ausdruck einer falsch verstandenen Regionalität. Zudem stellt auch der bereits begonnene Trend zur bundesweiten Preisangleichung die Forderung nach Regionalisierung der Einnahmen in Frage. In diesem Zusammenhang ist auch auf die Gültigkeit der Entscheidung des Bundesverfassungsgerichts aus dem Jahr $2005 \mathrm{zu}$ verweisen. Dort wird eindeutig festgestellt, dass regional unterschiedliche Preisniveaus und Versorgungsangebote nicht im länderübergreifenden RSA auszugleichen sind. Dies vor dem Hintergrund, dass zum einen Länder mit überdurchschnittlicher Versorgungsinfrastruktur, -qualität oder inanspruchnahme nicht von der gesamten GKV mitzufinanzieren sind, sondern haben hierfür selbst aufzukommen haben. Zum anderen verwirft das BVerfG den Ausgleich unterschiedlicher Kostenniveaus auch aus Gründen der Praktikabilität, da für die Umsetzung hochkomplizierte Datenerhebungen und die Beantwortung komplexer gesundheitsökonomischer Fragestellungen erfordert, die 
bis heute nicht gelöst sind. So fehlt es auch in der aktuellen Diskussion bislang weitestgehend an konkreten geeigneten Kriterien für eine Regionalisierung des aktuellen morbiditätsorientierten RSA. Zudem lässt sich ein regionaler Finanzausgleich über den RSA auch vor dem Solidarprinzip nicht rechtfertigen, da eine Alimentierung regionaler teurer Versorgungsinfrastruktur durch die Solidargemeinschaft nicht gewollt sein kann. Denkbar wäre allein eine Regionalisierung der Einnahmen durch die Erhebung regionaler Zusatzbeiträge der Kassen zur Finanzierung regionaler Besonderheiten in den Versorgungsstrukturen. Davon abgesehen, dass diese Lösung für eine bundesweite Kasse wie die TK systemfremd und die Umsetzung anfällig für wettbewerbliche Fehlanreize wäre, hat sich bereits gezeigt, dass die hierfür erforderliche Herauslösung einzelner Leistungsblöcke aus dem derzeitigen RSA (z.B. Verwaltungskosten, Leistungsbereiche) ein politisch brisantes Thema ist.

Angesichts der gerade erst erfolgten erfolglosen Debatte um mehr Steuerautonomie der Länder ist es fraglich, ob einer Regionalisierung der Einnahmen in dem nicht minder komplexen System der Gesetzlichen Krankenversicherung mehr Erfolg zuzutrauen ist. Anstelle einer Regionalisierung der Einnahmeseite sollte eine dezentrale Ausrichtung der Geschäftspolitik unter Wirtschaftlichkeitsaspekten vielmehr auf eine weitere Verbesserung der Effektivität und Effizienz dezentraler Angebote zielen, wie

- ein positives Kosten-Nutzen-Verhältnis regionaler Versorgungsangebote;

- eine bedarfsorientierte Regionalisierung von Versorgungsangeboten einerseits, bei gleichzeitiger Zentralisierung medizinischer Spezialangebote andererseits;

- mehr Handlungsspielräume der Kassen zur Gestaltung regionaler Versorgungsinnovationen;

- die Abschaffung des Zwangs zum flächendeckenden Angebot von $§ 73 b-$ Verträgen, die nach wie vor von den Kassen unabhängig von ihrem Nutzen angeboten werden müssen;

- verstärkte regionale Versorgungsanalysen, um Wirtschaftlichkeitspotenziale zu identifizieren und die Qualität sowie Effizienz der Angebote zu optimieren. 


\section{Regionalisierung als Chance und Herausforderung im Wettbewerb}

Ohne Frage ist eine für den Kunden spürbare regionale Präsenz einer Krankenkasse ein Vorteil im Wettbewerb um Kunden. Regionale Service- und Versorgungsstrukturen vermitteln Nähe, Unmittelbarkeit und Vertrautheit - emotional positiv besetzte Eigenschaften aus Sicht derzeitiger und künftiger Kunden. Diese Chance hat die TK erkannt und gestaltet vor diesem Hintergrund seit Jahren ihr regionales Profil. Gleichzeitig ist aber der Grad der Regionalisierung bei der TK als bundesweite Kasse begrenzt durch ihre im Vergleich zu regionalen Krankenkassen geringeren Marktanteile, die derzeit zwischen $18 \%$ und $5 \%$ variieren und ihre regionale Verhandlungs- und Gestaltungsmöglichkeiten einschränken. Ungeachtet der marktlichen Möglichkeiten ist unter dem Gesichtspunkt regionaler Mitgliederzuwächse zudem immer die Frage nach dem Gesamtnutzen für die TK und ihre Versicherten maßgeblich für die strategische Entscheidung, in welchen Regionen und für welche Zielgruppen welche Angebote eingerichtet werden. Aktuell ist der Markt durch Fusionen und durch Zusatzbeiträge ausgelöste Mitgliederwanderungen stark in Bewegung, sodass für den Wettbewerb innerhalb der GKV künftig Verschiebungen und veränderte Rahmenbedingungen für die regionale Versorgungsgestaltung zu erwarten sind. Derzeit stößt eine regional ausgerichtete Geschäftspolitik auch angesichts des uneinheitlichen aufsichtsrechtlichen Rahmens innerhalb der gesetzlichen Krankenkassen an ihre Grenzen. Die verschiedenen Aufsichtspraxen zwischen den jeweiligen Landesbehörden und dem Bundesversicherungsamts sorgen z.B. in Fragen des Abschlusses von Selektivverträgen oder der Genehmigung von Wahltarifen für unterschiedliche Voraussetzungen zwischen den Krankenkassen und stellen insbesondere eine Limitation im Wettbewerb mit regionalen Kassen dar. So ist es unverständlich, dass der Finanzausgleich einerseits zwischen allen Kassenarten unabhängig von ihren Aufsichtsbehörden stattfindet, andererseits aber Landesaufsichten über die Aufrechterhaltung insolventer Kassen entscheiden und dies von der Gesamtheit der Kassen mitzutragen ist. Hier ist dringend eine weitere Vereinheitlichung der Rahmenbedingungen erforderlich.

Kritisch zu betrachten in der aktuellen Regionalisierungsdebatte sind die Forderungen der Länder nach verstärktem Mitspracherecht in der Versorgungsplanung. Ohne Frage steht in Deutschland eine grundlegende Diskussion über eine nachhaltige Neuordnung der Versorgungsstrukturen im Sinne einer verstärkt bedarfsorientierten, sektorenübergreifenden Versorgung mit bundeseinheitlichen Kriterien an, wie mittlerweile politisch bereits durch das geplante Versorgungs- 
gesetz angekündigt wurde. Trotz der Neudefinition regionaler Versorgungsstrukturen muss aber eine Ausweitung der Länderkompetenzen differenziert betrachtet werden. Der geforderten Ausweitung von Landeskompetenzen bei der ambulant-ärztlichen sowie sektorenübergreifenden Versorgungsplanung, wie auch bei dem geforderten Beanstandungsrecht für Selektivverträge, ist entgegenzuhalten, dass gerade eine künftig erweiterte Versorgungsplanung nicht an den Landesgrenzen Halt machen darf. Ebenso sollte die wie bisher im stationären Sektor bestehende Trennung von Planungs- und Finanzierungsverantwortung nicht weiter auf weitere Leistungsbereiche ausgedehnt werden. Es darf bezweifelt werden, ob die Länder die selbst gesteckten Anforderungen an eine umfassende Planung dauerhaft erfüllen können angesichts der Tatsache, dass schon heute die Mittel fehlen, um den finanziellen Verpflichtungen in der Krankenhausfinanzierung nachzukommen. Gerade für die künftig erforderliche Versorgungsplanung über Landes- und Sektorengrenzen hinweg bietet dagegen eine bundesweite Kasse gute Voraussetzungen. Hier kann die TK dank ihrer schon heute guten Aufstellung in den Regionen, ihrer Erfahrung in der bundesweiten Identifizierung von Best-Practice-Modellen und der Entwicklung und Implementierung eigener Versorgungslösungen auf bewährte Strukturen und gewachsene Kompetenz zurückgreifen.

Vor allem vor dem Hintergrund der Versorgungsnotwendigkeiten ist eine stärkere Regionalisierung der Geschäftspolitik aus Sicht einer bundesweiten Kasse unter Umständen sinnvoll und auch machbar. Kernelement für ihr Gelingen und einen nachhaltigen Beitrag zum Nutzen des Unternehmens und seiner Versicherten bleibt aber ihre Einbettung in eine zentrale Strategie und Steuerung, denn richtig verstandene Regionalität kann aus Sicht einer bundesweit kalkulierenden Krankenkasse nur innerhalb eines einheitlichen Rahmens gelingen. 
Eberhard Wille and Klaus Knabner - 978-3-631-75550-1 Downloaded from PubFactory at 01/11/2019 03:39:43AM via free access 


\section{Gemeinsame Bedarfsplanung für die regionale Versorgung aus Sicht nie- dergelassener Ärzte}

\section{Carl-Heinz Müller}

\section{Vorwort}

Deutschland verfügt über eines der besten Gesundheitssysteme weltweit. Mit ganz wenigen Ausnahmen ist jeder krankenversichert. Das Leistungsniveau und der Leistungsumfang in der gesetzlichen Krankenversicherung ist hoch, der $\mathrm{Zu}$ gang zu Leistungen einfach. Wartezeiten sind in der Grundversorgung so gut wie unbekannt und in der spezialisierten Versorgung sind sie im internationalen Vergleich kurz. Jeder hat die gleichen Chancen auf Zugang zu Leistungen. Auch die Leistungsinhalte sind für alle gesetzlich Versicherten weitgehend gleich. Damit trägt das Gesundheitswesen in Deutschland erheblich zum sozialen Frieden bei.

\section{Demographischer Wandel, medizinischer Fortschritt und Ärztemangel}

Aber: das Gesundheitswesen in Deutschland steht vor großen Herausforderungen. Im Kern geht es darum, wie angesichts des demographischen Wandels und des medizinischen Fortschritts auch künftig jedem Bürger in Deutschland unabhängig von seinem Wohnort, Einkommen, sozialen Status und davon, bei welcher Krankenkasse er versichert ist, Zugang zu einer qualitativ hochwertigen, flächendeckenden medizinischen Versorgung garantiert werden kann. Der Bedarf an medizinischen Leistungen wird in einer immer älter werdenden Bevölkerung steigen, trotz eines Rückgangs der Bevölkerungszahl. Die Zunahme von chronischen Erkrankungen und Multimorbidität erhöht den Bedarf. Und dieser Bedarf verändert sich stark. Allerdings nicht einheitlich, sondern regional sehr unterschiedlich.

Tabelle 1: Anteil der Einwohner Deutschlands, die zum jeweiligen Jahresende 60 Jahre oder älter sind

\begin{tabular}{|c|c|}
\hline Jahr & Anteil in \% \\
\hline 1991 & 20,4 \\
\hline 2008 & 25,6 \\
\hline 2020 & 31,0 \\
\hline
\end{tabular}

Quelle: Statistisches Bundesamt 
Gleichzeitig gibt es bereits heute einen spürbaren Ärzte- und Fachkräftemangel, der in Zukunft dramatisch ansteigen wird. Bis zum Jahr 2020 scheiden aus der ambulanten medizinischen Versorgung 52.000 Ärzte ${ }^{1}$ aus, darunter 24.000 Hausärzte und 27.000 Fachärzte. 20.000 Chef- und Oberärzte scheiden aus der Krankenhausversorgung aus; bereits heute sind 5.500 ärztliche Stellen in Krankenhäusern nicht besetzt. Der Ersatzbedarf bis 2020 beläuft sich auf rund 80.000 kurativ tätige Ärzte - dabei sind die Morbiditätsverdichtung und der Trend zur Arbeitszeitverkürzung noch nicht berücksichtigt!

Abbildung 1: Ersatzbedarf und erwarteter Nachwuchs an (potenziell) kurativ tätigen Ärzten 2010 bis 2020

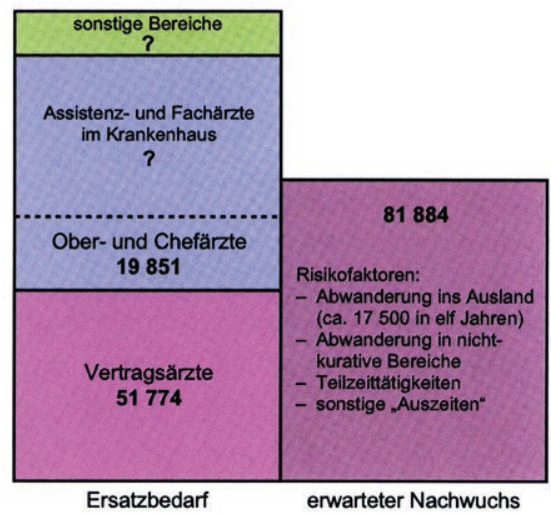

Quelle: Arztzahlstudie

Derzeit studieren insgesamt knapp 82.000 junge Menschen Medizin. Rein theoretisch könnten wir also damit den Ersatzbedarf weitgehend decken. Leider nur theoretisch, denn rund $18 \%$ der Studierenden beenden das Studium nicht. Von denjenigen, die es beenden, gehen ca. $12 \%$ nicht in die Patientenversorgung. Umfragen belegen darüber hinaus, dass viele über eine Abwanderung ins Ausland nachdenken. Von denen, die doch in Deutschland kurativ tätig werden wollen, wollen immer weniger in die Niederlassung gehen.

Die Gründe dafür kennen wir weitgehend: während des Studiums wird immer noch der mangelnde Praxisbezug und eine eher schlechte Betreuung durch die Dozenten beklagt. Zudem hat die nachwachsende Generation insgesamt andere

\footnotetext{
${ }^{1}$ Studie zur Altersstruktur und Arztzahlentwicklung, 5. aktualisierte Ausgabe www.kbv.de
} 
Erwartungen an ihre künftige Work-Life-Balance; kaum einer ist noch bereit, regelmäßig eine 55-Wochenstunde auf sich zu nehmen. Ein wichtiger Faktor liegt auch darin, dass sich der Anteil der Frauen in der Medizin kontinuierlich erhöht; heute sind mehr als 63 \% aller Studienanfänger Frauen. Damit steigt die Nachfrage nach Teilzeitangeboten und nach angestellter Tätigkeit auch in der ambulanten Versorgung.

\section{Künftig steht immer weniger ärztliche Arbeitszeit zur Verfügung}

Abbildung 2: Entwicklung des Anteils der Ärztinnen an den berufstätigen Ärztinnen und Ärzten

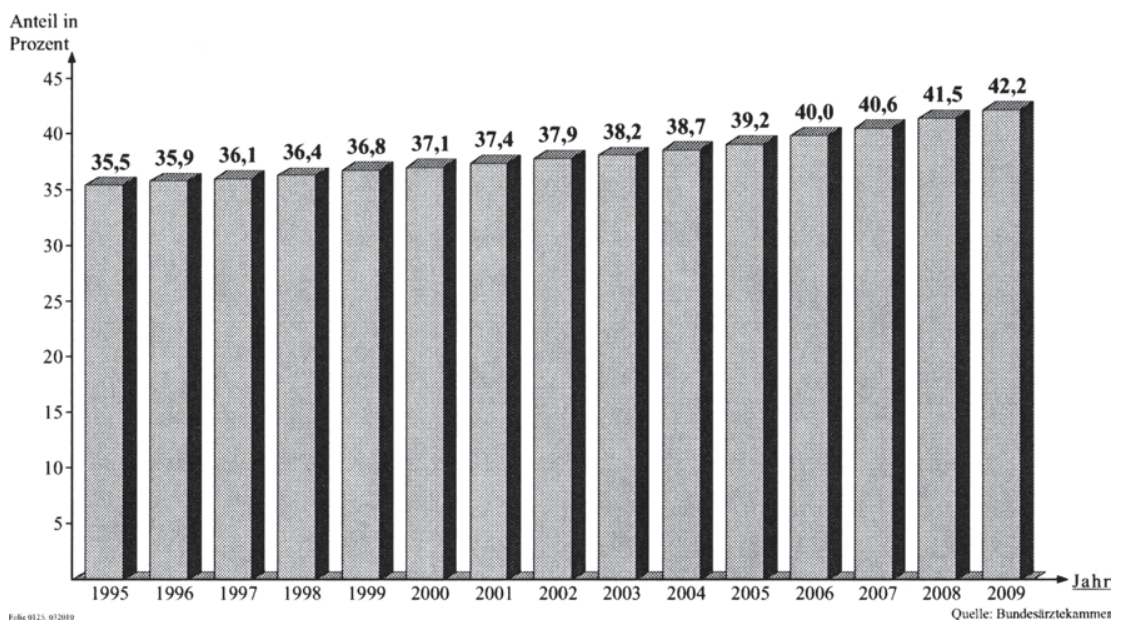

Quelle: Bundesärztekammer

Nach Angaben der Bundesagentur für Arbeit erbringen Frauen im Schnitt 72 $\%$ der Arbeitszeit der Männer. Mit steigendem Anteil an Frauen in der ärztlichen Versorgung sinkt damit die zur Verfügung stehende ärztliche Arbeitszeit. Hinzu kommt ein allgemeiner Trend zur Arbeitszeitverkürzung, der auch auf Ärzte zutrifft. Beide Faktoren erhöhen damit den Ersatzbedarf an Ärzten erheblich.

\section{Maßnahmenbündel gegen Ärztemangel erforderlich}

Es muss also alles daran gesetzt werden, die Attraktivität des Arztberufes zu erhöhen. Nur dann wir es gelingen, genügend Nachwuchs zu motivieren, in die Patientenversorgung zu gehen. Dafür ist ein ganzes Bündel von Maßnahmen 
erforderlich. Dazu zählt eine Optimierung des Medizinstudiums mit dem Ziel, eine Abwanderung der Studierenden und Absolventen in andere Tätigkeitsfelder zu minimieren ebenso wie die bessere Ausgestaltung der Weiterbildung zum Facharzt.

Im Zentrum dieser Anstrengungen müssen aber der Abbau der Bürokratie, eine angemessene Honorierung ärztlicher Leistungen und die endgültige Abschaffung des Regressrisikos bei verordneten Leistungen stehen. Das hat eine Umfrage der KBV und der Universität Trier bei über 12.000 Medizinstudierenden eindrucksvoll belegt. Auf die Frage danach, was sie von einer Niederlassung abhalten könnte, geben $63 \%$ der Studierenden das hohe finanzielle Risiko, $58 \%$ die hohe Bürokratie, $53 \%$ die unangemessen niedrige Honorierung und $50 \%$ die drohenden Regressforderungen (50\%) an. ${ }^{2}$

Eine selbständige freiberufliche Niederlassung als Hausarzt kommt nur für 38 $\%$ der Befragten in Frage, $62 \%$ schließen das für sich aus. Das liegt nicht an der Niederlassung als solcher, denn $74 \%$ der Befragten gaben an, dass eine Niederlassung als spezialisierter Facharzt für sie sehr wohl in Frage kommt. $54 \%$ aller Befragten lehnt eine Arbeit in kleinen Kommunen bis 2.000 Einwohner ab, am beliebtesten sind Städte zwischen 100.000 und 500.000 Einwohnern als möglicher Arbeitsort. Zusammengenommen bedeutet dieses Ergebnis: vor allem die Allgemeinmedizin muss gefördert werden, und da insbesondere die Niederlassung in ländlichen Gebieten. Denn ohne eine solche besondere Förderung wird es nicht gelingen, genügend Hausärzte für eine flächendeckende Versorgung zu gewinnen.

Die Umfrage belegt auch eindeutig, dass junge Ärztinnen und Ärzte deutlich flexiblere Berufsausübungsmöglichkeiten fordern. Sie wollen die Option haben, als angestellte oder selbständig tätige Ärzte sowie auf Teilzeittätigkeit haben egal ob in der ambulanten oder der stationären Versorgung. Sie legen verstärkt Wert auf Zusammenarbeit und Kooperation. Und sie wollen Familie, Freizeit und Beruf besser miteinander vereinbaren können. Bei der Wahl des Ortes spiele Faktoren wie die Arbeitsmöglichkeit für den Partner, die Kinderbetreuung, das schulische Angebot sowie das kulturelle und Freizeitangebot eine sehr große Rolle.

${ }^{2}$ Online-Umfrage von KBV und Universität Trier im Frühsommer 2010 bei ca. 12.500 Medizinstudierenden in ganz Deutschland, www.kbv.de 


\section{Veränderte Bedürfnisse erfordern veränderte Konzepte}

Die skizzierten veränderten Bedürfnisse der Patienten und der jungen Ärzte erfordern veränderte Rahmenbedingungen in der medizinischen Versorgung. Dazu gehören neue Versorgungsformen, sektorenübergreifende Behandlungspfade, die Förderung von Kooperationen in der ambulanten und sektorenübergreifenden Versorgung und mit anderen Gesundheitsfachberufen. Notwendig ist auch eine Neuordnung der Bedarfsplanung in der ambulanten ärztlichen Versorgung.

\section{Neuordnung der Bedarfsplanung erforderlich}

Denn neben dem bereits beschriebenen generellen Problem des Ärztemangels gibt es auch ein Problem mit der Verteilung ärztlicher Kapazitäten. Es ist unbestritten, dass Ballungsgebiete teilweise überversorgt sind während in ländlichen Gebieten der Mangel an grundversorgenden Haus- und Fachärzten bereits deutliche Versorgungsengpässe hervorruft. Eine sinnvolle Allokation knapper ärztlicher Ressourcen ist daher ein enorm wichtiger Baustein zur Sicherstellung einer flächendeckenden, qualitativ hochwertigen Versorgung auch in Zukunft.

Zunächst muss die Frage danach beantwortet werden, ob eine Bedarfsplanung überhaupt noch notwendig ist. In der Marktwirtschaft gilt grundsätzlich: Angebot und Nachfrage sorgen für eine effiziente Allokation vorhandener Ressourcen. Für das Gesundheitswesen kann das aber nur sehr eingeschränkt gelten. Denn neben den marktwirtschaftlichen Mechanismen müssen in der Gesundheitsversorgung auch soziale Ziele wie Versorgungssicherheit und Versorgungsgerechtigkeit verfolgt werden. Um diese Ziele zu erreichen, bedarf es auch weiterhin einer Bedarfsplanung. Diese muss aber auf die veränderten Bedürfnisse ausgerichtet und neu gestaltet werden.

Das lässt sich am Beispiel Berlins zeigen. Dort wurde die bis dahin kleinräumige Bedarfsplanung auf Bezirksebene zum 30.06.2003 abgeschafft. In der Folge konnten die Ärzte ihren Praxissitz innerhalb Berlins über die Bezirksgrenzen hinweg beliebig verlegen. Die Konsequenz der Aufhebung der kleinräumigen Bedarfsplanung in Berlin war, dass die Vertragsärzte nun unabhängig von den Bedarfsfestlegungen auf der Ebene der Bezirke ihren Standort über die Bezirksgrenzen hinweg verlagern konnten. Im Ergebnis sind die Ärzte aus jenen Bezirken, in denen sich die Versorgungslage (gemessen an der Relation Ärzte je 1000 Einwohner) schlechter darstellte, in die Bezirke mit einer guten Versorgungslage 
umgezogen. Eine Abschaffung der kleinräumigen Bedarfsplanung sorgte hier also für eine Umverteilung der Ärzte entgegen der eigentlichen Versorgungsbedarfe.

Das Beispiel zeigt nicht nur, dass Bedarfsplanung für eine sinnvolle Steuerung knapper Ressourcen notwendig ist, sondern auch, dass die Bedarfsplanung neu geordnet werden muss. Die heutige Bedarfsplanung entspricht den Anforderungen an eine zeitgemäße Versorgungsplanung schon lange nicht mehr. Die Planungsbereiche sind zu großräumig, die Einteilung der Arztgruppen ist nicht differenziert genug und die Versorgungslage in benachbarten Planungsbereichen wird nicht berücksichtigt. Die Bedarfsplanung, wie wir sie heute kennen, entstammt der Zeit, als von einer Ärzteschwemme die Rede war und die Möglichkeiten zur Niederlassung begrenzt werden sollten. Sie ist viel zu grob, um kleinräumige Versorgungsbedarfe zu berücksichtigen. Außerdem berücksichtigt sie die Morbiditätsentwicklung in einer Region nicht. Sie ist nicht prospektiv ausgerichtet und für die Bekämpfung von Unterversorgung völlig ungeeignet.

\section{Das KBV-Konzept einer bedarfsorientierten, sektorenübergreifenden Ver- sorgungsplanung}

Die KBV hat dafür ein aussichtsreiches Konzept entwickelt: Grundlage ist der tat-sächliche Versorgungsbedarf in der betrachteten Region. Dazu werden neben Alter und Geschlecht der Wohnbevölkerung auch deren Morbidität sowie erwartete Morbiditätsveränderungen berücksichtigt. Die Versorgungsebenen werden ebenfalls neu geordnet. Künftig soll zwischen einer haus- und fachärztlichen Grundversorgung, die flächendeckend wohnortnah im Kollektivvertrag organisiert wird und einer spezialisierten fachärztlichen Versorgungsebene, die vorrangig in Berufsausübungsgemeinschaften in Mittel- und Ober-Zentren vorgehalten und sektorenübergreifende geplant wird, differenziert werden.

Die festgestellten regionalen Versorgungsnotwendigkeiten werden in einem neu zu bildenden Regionalverbund auf Landesebene bewertet, der verpflichtende Vorgaben jeweils für den ambulanten und stationären Versorgungsbereich macht - unter Wahrung der jeweiligen Sicherstellungskompetenzen. Hierzu bilden die Kassenärztliche Vereinigung, die Landesärztekammer, die Landeskrankenhausgesellschaft und die zuständige Landesbehörde für Krankenhausplanung für den Bereich der Kassenärztlichen Vereinigung eine Arbeitsgemeinschaft zur Koordinierung der Versorgungssteuerung im Lande. 
Abbildung 3: Arbeitsgemeinschaft für sektorübergreifende Versorgungsplanung

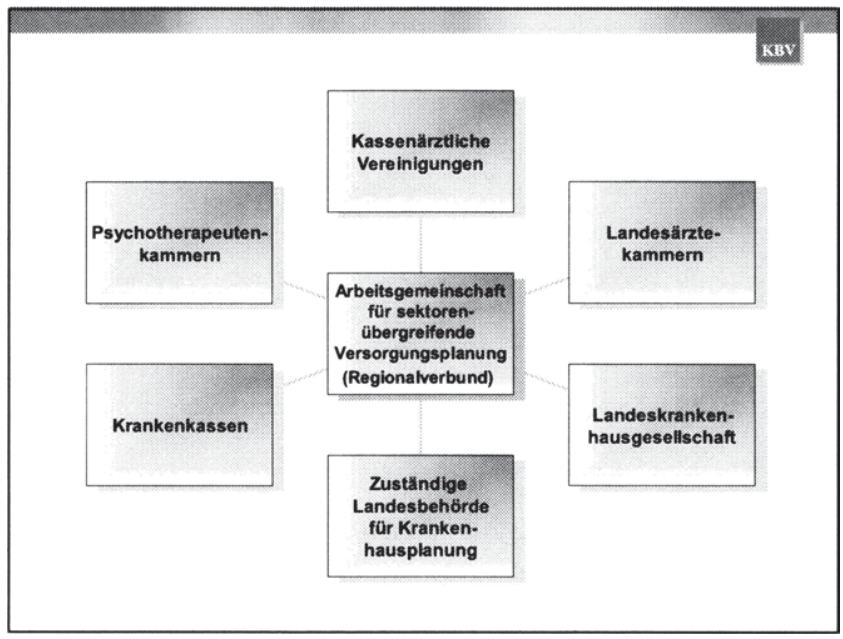

Damit wird von der bisherigen abgeschotteten sektoralen Betrachtung des ambulanten und stationären Bereichs abgegangen. Dies bedeutet, dass sich die bisherige Schnittstellenproblematik etwa der ambulanten Operationen gem. $\S$ $115 \mathrm{~b}$ SGB V, der ambulanten Behandlung im Krankenhaus gem. § 116b SGB V sowie der Psychiatrischen Institutsambulanzen gem. § 118 SGB V abgelöst wird durch eine strukturierte Beziehung der spezialisierten fachärztlichen Versorgungsebene zum ambulanten und stationären Bereich. Der Versorgungsauftrag beispielsweise für die rheumatologische Versorgung als spezialisierte fachärztliche Versorgung könnte nach Maßgabe des Regionalverbundes daher sowohl im vertragsärztlichen Versorgungsbereich als auch im Krankenhaus als ambulante Behandlung erfüllt werden, wenn die entsprechenden Kapazitäten und Qualifikationen vorhanden sind.

Hierfür ist die Angleichung des Leistungskatalogs, der Qualitätsanforderungen sowie der Vergütung in der fachärztlichen spezialisierten Versorgung zwingend erforderlich.

Für die wohnortnahe Grundversorgung werden nach den Kriterien Versorgungsbedarf, Wegezeiten und wirtschaftliche Tragfähigkeit Arztsitze identifiziert, deren Besetzung die regionale Versorgungsnotwendigkeit optimal abbil- 
det. Potenzielle Standorte werden nur dann in die Planung aufgenommen, wenn von ihnen eine ausreichende Anzahl an Einwohnern in ihrem Einzugsbereich versorgt würde. Zudem dürfen umliegende Standorte nicht in ihrer Existenzfähigkeit beeinträchtigt werden. Daher werden auch die sogenannten Mitversorgereffekte in die Betrachtung einbezogen. Hierdurch werden ein ausreichende Wirtschaftlichkeit und gleichzeitig die Qualität der medizinischen Leistungen (Mindestmengen) sichergestellt. Die nach diesen Kriterien identifizierten Versorgungssitze werden vom Zulassungsausschuss ausgeschrieben. Bewirbt sich kein Arzt darum, kann die KV selbst Ärzte anstellen und/oder in Zusammenarbeit mit den Kommunen Filialpraxen einrichten, die zu bestimmten Zeiten von unterschiedlichen grundversorgenden Ärzten besetzt werden. Diese können ggf. auch in einem MVZ angestellt sein. In solchen Filialpraxen können besonders qualifizierte nicht-ärztliche Mitarbeiter erster Ansprechpartner für Patienten sein und auch delegierbare Leistungen, wie etwa bestimmte Hausbesuche, erbringen. Bei Bedarf können diese Ärzte auch die Versorgung in Pflegeheimen übernehmen.

Ein Strukturfonds, der bei den Kassenärztlichen Vereinigungen gebildet wird, stellt die dafür notwendigen Mittel zur Verfügung. Dabei sollen vor allem $\mathrm{Zu}$ schüsse zur Finanzierung von Praxen und der Organisation des Notfall- und Bereitschaftsdienstes sowie von Arztstationen in strukturschwachen Gebieten gegeben werden. Aus diesem Fonds können auch Praxen in überversorgten Gebieten aufgekauft und stillgelegt werden, sofern diese für die Versorgung nicht benötigt werden.

Kassenärztliche Vereinigungen können darüber hinaus zur Verbesserung der Kooperation zwischen ambulanter und stationärer Versorgung gemeinsam mit Krankenhäusern oder alleine Medizinische Versorgungszentren gründen. Vertragsärzte, Psychotherapeuten und Krankenhausärzte übernehmen darin als Brückenfunktionen u.a. die nachstationäre ambulante Versorgung, die Errichtung von Notfallambulanzen in unterversorgten Gebieten sowie die Aufgaben der Praxiskliniken.

\section{Gesundheitspolitische Diskussion hat eingesetzt}

Das KBV-Konzept zur Neuordnung der Versorgungsplanung bietet aussichtsreiche Lösungsansätze für bestehende und künftige Probleme. Es ist innovativ und zukunftsorientiert und trägt zu einer effizienten und sozial ausgeglichenen Allo- 
kation knapper finanzieller und personeller Ressourcen bei. Damit leistet die KBV auch einen wichtigen Beitrag zur nunmehr einsetzenden Diskussion um das geplante GKV-Versorgungsgesetz. 
Eberhard Wille and Klaus Knabner - 978-3-631-75550-1 Downloaded from PubFactory at 01/11/2019 03:39:43AM via free access 
Flexibilisierung in der stationären und ambulanten Versorgung im Rahmen eines öffentlichen Krankenhausverbundes

Joachim Bovelet

Sehr geehrte Damen und Herren,

\section{Vivantes Netzwerk für Gesundheit}

Vivantes ist als öffentlicher Krankenhausverbund das größte kommunale Krankenhausnetzwerk in Deutschland.

Im Vivantes Netzwerk wurden durch Beschluss des Abgeordnetenhauses von Berlin per 1.Januar 2001 zehn städtische Krankenhäuser zusammengefasst.

Mittlerweile sind es 9 Krankenhäuser, die in 6 von 12 Bezirken Berlins lokalisiert sind. Teilweise handelt es sich um die ältesten Krankenhäuser in Berlin überhaupt, wie die Krankenhäuser im Friedrichshain und am Urban, die auf mehr als 100 Jahre Vergangenheit zurückblicken können.

\section{Rechtsform GmbH}

Mit der gewählten GmbH Rechtsform verfolgte der alleinige Gesellschafter, das Land Berlin, das Ziel, bisher defizitäre Strukturen der städtischen Krankenhäuser zu optimieren und eine Inanspruchnahme des Landeshaushalts zu vermeiden.

Die Vision der damaligen Geschäftsführung sah vor, die neu entstandene Vivantes $\mathrm{GmbH}$ zu einem führenden Dienstleistungsunternehmen im Gesundheitswesen in Deutschland zu entwickeln.

\section{Einführung DRG}

Wie Sie wissen, hat es seit 2001 mehrere Gesetzesänderungen im Gesundheitswesen allgemein und insbesondere im Krankenhausbereich gegeben.

Mit der Einführung der DRG als Entgelt für bestimmte Krankenhausleistungen wurde im Krankenhaussektor ein ökonomisches Fundament gelegt, das die bisherige Produktionsweise im Krankenhaus in Frage stellte. 
Statt der Tagespflegesätze und der dadurch induzierten längeren Verweildauer, besteht der Anreiz beim DRG-System darin, bei gegebenem Preis die Kosten, also insbesondere die Verweildauer, zu senken.

\section{Prozessanpassungen}

Dieser ökonomische Paradigmenwechsel erzwang auch eine Veränderung der bisherigen Produktionsweise im Krankenhaus. Die Veränderung der betrieblichen Arbeits-und Behandlungsprozesse forderte den Krankenhausbeschäftigen vieles ab.

Auch die bisher angewandte Technologie zur Steuerung dieser Arbeits-und Behandlungsprozesse musste durch eine moderne, den neuen Erfordernissen gerecht werdende EDV ersetzt werden.

Nur dadurch war überhaupt ein Controlling der neuen Prozesse möglich und nur damit wiederum ließ sich dokumentieren, inwieweit die Ziele erreicht bzw. nicht erreicht wurden und wo Gegensteuerungen notwendig waren.

\section{Flexibilität \& Innovation}

Aus der vorstehenden Skizze wird deutlich, dass die notwendigen Anpassungsprozesse im Krankenhaus allen Beteiligten eine große Flexibilität abverlangten und weiterhin abverlangen.

Langjährige eingefahrene Abläufe wurden durch neuer Arbeits-und Behandlungsprozesse abgelöst und es zeigte sich in der Folge, dass auch das etablierte Neue eine im Vergleich zu früheren Zeiten immer kürzere Halbwertzeit hatte.

Wie in anderen gesellschaftlichen Sektoren verkürzen sich auch im Krankenhausbereich die Innovationszyklen und erfordern gerade in Zeiten eines verschärften Wettbewerbs flexible Anpassungen, um in diesem Wettbewerb mit der Konkurrenz bestehen zu können.

\section{Integration 9 Krankenhäuser zu Vivantes}

Die zu Beginn skizzierten Umstände der Gründung der Vivantes Netzwerk für Gesundheit $\mathrm{GmbH}$, in der 9 bisher selbständig agierende städtische Krankenhäuser in eine privatrechtliche Gesellschaftsform eingebunden wurden, geben Ihnen wohl einen ungefähren Eindruck von der gewaltigen Aufgabe, die das Manage- 
ment und die Mitarbeiter zu stemmen hatten, um aus diesen, zum Teil stark defizitären arbeitenden Einzelkrankenhäusern, einen Konzern zu bilden.

Innerhalb dieses Konzerns galt es nun die Stärken der Einzelhäuser durch Konzentration und Zentralisation zu bündeln und Schwachstellen bei der Wirtschaftlichkeit der Leistungserbringung zu beseitigen.

\section{Vernetzung stationär - ambulant}

Obgleich das Kerngeschäft von Vivantes vorrangig im stationären Bereich liegt, war aber mit der Gründung des Konzerns zugleich klar, dass eine alleinige Beschränkung des Vivantes-Konzerns auf den stationären Bereich, der in der gesundheitspolitischen Diskussion immer stärker thematisierten Vernetzung des stationären und des ambulanten Sektors nicht gerecht werden würde.

Neben dem schon bei der Vivantes Gründung bestehenden Geschäftsfeld Senioren, wo Vivantes mit fast 2000 vollstationären Pflegeplätzen der größte Einzelanbieter in Berlin ist, hat Vivantes 2002 eine Tochtergesellschaft für die ambulante medizinische Rehabilitation gegründet.

Damit wurden die stationäre Versorgungsangebote von Vivantes um den Bereich der ambulanten Rehabilitation ergänzt und die Behandlungskette für Patienten innerhalb des Konzerns erweitert.

\section{MVZ 2006}

Durch die Gesetzgebung wurde die Möglichkeit eröffnet, durch krankenhausgetragene Medizinische Versorgungszentren weitergehender als bisher im ambulanten Sektor tätig zu sein. Vivantes hat mit der Gründung einer Tochtergesellschaft in 2006 diesen Schritt umgesetzt.

Der Wettbewerb unter den Krankenhäusern dreht sich künftig nicht mehr nur allein um stationäre Patienten, sondern auch um den Einfluss im ambulanten Bereich.

Krankenhäuser, die ein vernetztes Angebot in beiden Sektoren anbieten können, sind sowohl für stationäre wie ambulante Patienten attraktiv. Beide Patientengruppen wissen, dass ein gegebenenfalls notwendiger Behandlungsübergang in 
den jeweils anderen Sektor durch eine stringente und qualitätsgesicherte Versorgungskette des Krankenhauses gewährleistet werden kann.

\section{Jahre Vivantes -Sanierung}

Vivantes feiert im nächsten Jahr sein 10jähriges Bestehen. Dazu muss aber auch gesagt werden, dass die Anfangsjahre sehr schwierig waren.

Vivantes gelang es durch erfolgreiche Reduktion der Sach-und Personalkosten sowie durch Zentralisierung und Einsparungen in der Verwaltung und den medizinischen Servicebereichen, erstmals in 2004 ein positives Jahresergebnis auszuweisen. In den Folgejahren hatte Vivantes weiterhin positive Jahresergebnisse erzielt und wird dieses Ziel aller Voraussicht nach auch für 2010 erreichen.

Diese Ergebnisse verdanken sich nicht zuletzt der Flexibilität der Mitarbeiter von Vivantes. Diese waren bereit, um den Preis einer Beschäftigungsgarantie bis 2014, auf einen Teil ihres Gehalts zu verzichten.

\section{Wachstumskurs}

Es ist auch den Mitarbeitern von Vivantes zu danken, dass die Patientenzahl in den Krankenhäusern von Vivantes seit mehreren Jahren und insbesondere in den letzten beiden Jahren beträchtlich gesteigert werden konnte.

Die Akzeptanz des Vivantes-Konzerns bei der Berliner Bevölkerung und auch im Umland zeigt sich darin, dass das Patientenzahlwachstum signifikant über dem Fallzahlwachstum in allen Berliner Krankenhäusern lag.

\section{Investitionen}

Trotz der beschriebenen positiven Entwicklung des Vivantes Konzerns, ist weiterhin eine flexible Anpassung an die sich ändernden Rahmenbedingungen notwendig.

Der Kernpunkt im Wettbewerb mit anderen Krankenhäusern auch im ambulanten Bereich sind die Investitionsmöglichkeiten eines Unternehmens.

Auch wenn Vivantes Jahresüberschüsse ausweist, ist die Umsatzrentabilität zu klein, um größere Investitionen aus Eigenmitteln zu finanzieren. 
Über die fehlenden Investitionsmittel im Rahmen der dualen Finanzierung sind hier keine weiteren Ausführungen notwendig.

Die Vivantes Geschäftsführung hat sowohl an der Leistungsmengen-und auch der Kostenschraube bisher erfolgreich gedreht und wird dies auch unter Benchmarking Aspekten weiter tun.

Darüber hinaus dienen auch strategische Partnerschaften, wie wir sie z.B. mit der Charite beim gemeinsamen Laborprojekt anstreben, dem Ziel, zusätzliche Effizienzgewinne zu realisieren.

\section{Finanzierung}

Es bleibt aber, insbesondere angesichts der noch existierenden baulichen Strukturen einzelner Vivanteshäuser, die unabweisbare Notwendigkeit von Ersatzbzw. Neuinvestitionen.

Da vom alleinigen Gesellschafter von Vivantes, also dem Land Berlin, in dieser Hinsicht nicht viel zu erwarten ist und Vivantes die erforderlichen Mittel nicht selbst erwirtschaften kann, muss Vivantes einen Weg zur Beschaffung von Investitionsmittel auf dem Kapitalmarkt suchen.

Hierzu ist es allerdings notwendig, dass sich der bisherige Alleingesellschafter der Vivantes Netzwerk für Gesundheit $\mathrm{GmbH}$ in dieser Frage ebenfalls flexibel zeigt.

Dann könnte auch geprüft werden, ob z.B. eine kommunale Aktiengesellschaft ein geeigneter Weg wäre, um auf dem Kapitalmarkt die benötigten Investitionsmittel zu beschaffen.

\section{Zusammenfassung}

Lassen Sie mich zusammenfassen:

- Vivantes ist als kommunaler Konzern 2001 gegründet worden, um aus defizitären städtischen Krankenhäusern im Verbund bzw. Netzwerk ein wirtschaftlich tragfähiges Unternehmen zu entwickeln.

- In dem fast 10jährigen Bestehen des Vivantes Konzerns ist es durch Zentralisierung und durch Reduktionen bei den Sach-und Personalausgaben sowie 
durch die Erweiterung des Vivantes Portfolios im ambulanten Bereich gelungen, Jahresüberschüsse zu erzielen.

Die Mitarbeiter von Vivantes haben dabei ein hohes Maß an Flexibilität und Identifikation mit dem Unternehmen gezeigt.

- Um auch in der nächsten Dekade erfolgreich zu bestehen, muss Vivantes die Möglichkeit eröffnet werden, neben den weiterhin notwendigen Rationalisierungen, sich die Mittel für Zukunftsinvestitionen auf dem Kapitalmarkt zu beschaffen.

Vielen Dank für Thre Aufmerksamkeit! 


\section{Chancen und Grenzen der Individualisierten Medizin}

Was braucht unser System, um zukünftiges Wissen optimal nutzen zu können

\section{Hagen Pfundner}

Für das Geschäftsmodell forschender Pharmaunternehmen ist eine hohe Forschungs- und Entwicklungs-Produktivität ein elementares Wettbewerbsinstrument. Die Personalisierte Medizin bietet eine Chance, um die Gesundheitsversorgung in seiner Qualität besser, sicherer und effizienter zu machen. Die Personalisierte Medizin wird eine Systemrelevanz haben, weil sie neben den Vorteilen bezogen auf den Erkenntnisgewinn für eine bessere Wirksamkeit und Verträglichkeit von Arzneimitteln auch zu einer Veränderung der Vergütung von Gesundheitsleitungen führen wird.

\section{Übersicht}

Die Personalisierte Medizin steht im Mittelpunkt der Innovationsstrategie für Roche und ist ein wichtiges Instrument, um die Erfolgsrate bei der Entwicklung von Arzneimitteln zu erhöhen und den Patienten gezielter klinisch differenzierte Gesundheitslösungen anzubieten. Aktuelle Herausforderungen und Entwicklungen machen darüber hinaus eine Neuorientierung aller Leistungserbringer einschließlich der Beteiligten in der Politik hin zur Personalisierten Medizin erforderlich. Die Chancen, die sich bereits heute durch die therapeutische Stratifizierung und Fokussierung auf kleine Patientenpopulationen ergeben, liegen auf der Hand und konkrete Beispiele für individualisierte Therapiekonzepte liegen vor. Die Identifikation von Biomarkern und eine begleitende Diagnostik ist hierbei eine wesentliche Voraussetzung für die weitere Evolution der Personalisierten Medizin. Aus den hohen Erwartungen, die an die zukünftigen Konzepte gestellt werden, leiten sich auch Konsequenzen für eine Neuausrichtung der Forschung \& Entwicklung (F\&E) ab. Damit sich die Erwartungen in den Nutzen der Personalisierten Medizin für alle Beteiligten im Gesundheitswesen erfüllt, müssen allerdings wichtige Rahmenbedingungen erfüllt sein.

Das populationsbasierte „one size fits all“ Geschäftsmodell von Pharma basiert auf Innovationen, die sich durch gute Wirksamkeit und möglichst geringe Nebenwirkungen in der „breiten Anwendung“ auszeichnen. Dieses Modell wird auch zukünftig verfolgt werden. Durch sinkende F\&E-Produktivität, einen steigenden Verdrängungswettbewerb und die zunehmenden Anforderungen an den 
Nachweis des klinischen und ökonomischen Nutzens müssen forschende Unternehmen allerdings neue Wege gehen.

Sind neue therapeutische Ansatzpunkte - für bereits erforschte oder bisher noch unerforschte Krankheiten - identifiziert, erfordert die Selektion geeigneter Arzneimittelkandidaten den Einsatz hochkomplexer und kostenintensiver Technologien, damit die ersten Entwicklungsstufen bis zur ersten Prüfung am Menschen erfolgreich durchlaufen werden können. In der anschließenden klinischen Prüfung gelangt derzeit nur noch einer von 14 erstmals am Menschen erprobten Wirkstoffen auf den Markt - 13 Testkandidaten scheitern. Auch wenn sich die Anzahl an Versuchen in Phase I und II in den letzten 10 Jahren fast verdoppelt hat, ist die Erfolgsquote heute nur noch halb so hoch.

Gründe für das Scheitern eines Arzneimittelkandidaten auf der Wegstrecke von der klinischen Prüfung bis zur Zulassung können einerseits substanzbezogen sein, wie beispielsweise fehlende Wirksamkeit oder unerwartet auftretende Nebenwirkungen. Andererseits können externe Gründe wie veränderte Marktbedingungen einschließlich gesundheitspolitischer und regulatorischer Rahmenbedingungen Ursachen für fehlende Marktchancen des Arzneimittels sein. Oftmals ist zu Beginn der Arzneimittelentwicklung noch nicht absehbar, ob und inwiefern sich zukünftige Kostendämpfungsmaßnahmen auf das Marktpotential des Arzneimittels auswirken. Von der Entdeckung bis zur Markteinführung eines neuen Arzneimittels vergehen im Durchschnitt 12 Jahre.

Medizinisch-technische Innovationen führen genauso wie Lebensstiländerungen zu steigender Lebenserwartung der Menschen. Diese wiederum führt auch zu einer steigenden Nachfrage nach medizinisch-technischen Leistungen und Innovationen im Alter.

Bei Analyse der Umsatzveränderungen im Arzneimittelmarkt Deutschland zeigt sich, dass die treibenden Faktoren des Ausgabenzuwachses für Arzneimittel in erster Linie der medizinische und therapeutische Bedarf der Patienten sind. In Deutschland sind die Gesundheitsausgaben an den Faktor Arbeit gekoppelt. Durch die Verlängerung der produktiven (arbeitsfähigen) Lebenszeit trägt die Pharmaindustrie somit direkt zur Stärkung der Gesundheitskassen bei. 
Das gegenwärtige Geschäftsmodell der pharmazeutischen Industrie verfolgt einen populationsbasierten Therapieansatz - in der Annahme, dass Patienten mit identischer Diagnose auf das Arzneimittel gleich gut ansprechen.

Seit langem ist allerdings bekannt, dass Patienten mit identischer Diagnose auf die Behandlung mit dem gleichen Arzneimittel unterschiedlich ansprechen können. Während sich die Behandlung für den einen Patienten als gut wirksam erweist, kann sie bei einem anderen Patienten keinen Nutzen erzielen. Mögliche Ursachen sind individuelle Merkmale des Patienten oder krankheitsspezifische Faktoren, welche die Wirkungsweise von Medikamenten beeinflussen. Wenn aber alle Patienten mit derselben Diagnose die gleiche Therapie erhalten (populationsbasierter Ansatz), können weder individuelle Patienten-Merkmale noch krankheitsspezifische Faktoren adressiert werden. Daten zufolge erzielt bis zu jedes dritte eingesetzte Arzneimittel nicht die gewünschte Wirkung, so dass nur etwa zwei Drittel aller Patienten von ihrer medikamentösen Therapie profitieren. ${ }^{1}$ Darüber hinaus können unerwünschte Nebenwirkungen die Therapie nachteilig beeinflussen. Nach Schätzungen von Experten sterben in Deutschland jährlich ca. 16.000 - 25.000 Menschen an den Nebenwirkungen von Arzneimitteln, ${ }^{2}$ in den USA einer Meta-Analyse zufolge 76.000 - 137.000 Patienten. ${ }^{3}$ Dies ist eine unzufrieden stellende Situation: Für Patienten und Ärzte, weil weder Wirkung noch Nebenwirkung präzise kalkuliert werden können; und für Kostenträger, weil Behandlungen bezahlt werden, deren Wirksamkeit und Sicherheit in vielen Fällen suboptimal ist. Für die Pharmaindustrie sind zu einer Zeit, in der die klinischen und regulatorischen Hürden für die Markteinführung neuer Medikamente höher werden, zwei Aspekte wichtig: Reduktion der Kosten für breit und groß angelegte klinische Studien und Verkürzung der Studiendauer. Die Behandlungsoption genauer auf definierte Patientenpopulationen zuzuschneiden und damit besser einschätzen zu können, welche Patienten höchstwahrscheinlich auf eine bestimmte Therapie ansprechen werden, adressieren beide Aspekte.

Personalisierte Gesundheitslösungen sind in gewisser Weise nichts Neues. Schon immer waren Ärzte bemüht, Behandlungen auf den Patienten abzustimmen. Seit Jahrzehnten verwenden Ärzte Blutzuckertests, um den Insulinbedarf

\footnotetext{
${ }^{1}$ Spear, BB. u.a.: Clinical application of pharmacogenetics. Trends Mol Med. 2001; 7(5): 201-204.

${ }^{2}$ Süddeutsche Zeitung. Medikamente und Nebenwirkungen. 9.11.2007

${ }^{3}$ Lazarou, J. u.a.: Incidence of adverse drug reactions in hospitalized patients: a meta-analysis of prospective studies. JAMA 1998; 279(15):1200-5.
} 
von Patienten mit insulinabhängigem Diabetes zu bestimmen. Neuere Beispiele für eine von der Diagnostik geleiteten Therapie sind die Kontrolle der Viruslast und die Ermittlung des Virus-Genotyps bei Patienten, die mit dem HI- oder Hepatitis C-Virus infiziert sind. Diese Tests messen die virale Belastung im Blut des Patienten und können Resistenzen der Viren gegen bestimmte Arzneimittel feststellen. Anhand dieser Informationen können Ärzte besser entscheiden, wie lange und wie aggressiv die Infektion eines Patienten zu behandeln ist, welche Kombination von Arzneimitteln verabreicht werden soll, und wann das Medikament gewechselt oder die Dosierung verändert werden muss.

Völlig neue Perspektiven eröffnen sich durch die Dynamik des wissenschaftlichen Fortschritts im Bereich der Molekularbiologie, insbesondere durch die Entschlüsselung des menschlichen Erbguts. Durch die Erforschung der Biologie und der Behandlung von Krankheiten auf molekularer Ebene werden die Ursachen von Krankheiten zunehmend genauer bekannt. Die Einblicke in molekulare Prozesse, die Genomik und Proteomik sowie die Gentechnologie haben in den letzten Jahren zu einer Wissensexplosion geführt, die neue Möglichkeiten für wirksamere Behandlungen eröffnet. In der Arzneimittelforschung kommen neue Technologien zum Einsatz, um Moleküle im Körper zu finden, die sich gut als Zielstrukturen („Targets“) für Medikamente eignen könnten. Beispielsweise bedeutet das in der Krebstherapie Strukturen im Tumor zu finden, die für diesen Tumor typisch sind und im gesunden Gewebe nicht oder nur in geringem Masse auftreten. Gelingt das, können gezielt Arzneimittel gegen diese Strukturen entwickelt und nebenwirkungsarm eingesetzt werden.

Mit der molekularen Diagnostik rückt ein völlig neuer Ansatz ins Blickfeld des Interesses, der die therapeutische Praxis nachhaltig beeinflusst. Krankheiten sind multifaktoriell bedingt, und neben Umweltfaktoren, Ernährung und Lebensstil spielt die genetische Prädisposition eine wichtige Rolle bei der Entstehung von Krankheiten. Innerhalb einer großen Patientenpopulation können so bestimmte Gruppen identifiziert werden, die verschiedene Behandlungsansätze benötigen. Diese Stratifizierung der Patienten bildet den Kern der Personalisierten Medizin, indem Patientengruppen mit unterschiedlichen (z.B. genetischen) Merkmalen auch unterschiedlich behandelt werden - durch unterschiedliche Medikamente oder auch durch unterschiedliche Dosierungen des gleichen Arzneimittels. Die therapeutische Stratifizierung erfolgt mit Hilfe von sogenannten Biomarkern. Zunächst ist ein Biomarker allgemein definiert als eine objektive Messgröße zur Bewertung von normalen biologischen Prozessen, von pathologi- 
schen Prozessen, von pharmakologischen Reaktionen auf eine therapeutische Intervention oder von Reaktionen auf präventive oder andere Gesundheitsinterventionen. Bekannte, klassische Biomarker sind z.B. der Blutdruck oder der Blutzuckerspiegel.

Erst die wissenschaftlichen Fortschritte im Bereich der Molekularmedizin haben seit den $80 \mathrm{er}$ Jahren ermöglicht, sehr viel aussagekräftiger und präziser in der Biomarkerentwicklung voranzuschreiten. Hierbei werden neue, aus der Genom- und Postgenomforschung und molekularen Medizin hervorgehende Biomarker sowie entsprechende Verfahren zu ihrer Messung, z.B. bildgebende Verfahren, herangezogen. Die „Therapeutische Stratifizierung mit Hilfe von Biomarkern" heißt, Arzneimittel aufgrund genetischer Profile oder spezifischer Krankheitsmuster genauer auf ihre Wirkung bei bestimmten Patientengruppen zuzuschneiden. Mit dem Biomarker sollen aus dem gesamten Patientenkollektiv diejenigen Personen herausgefiltert werden, die eine größere Wahrscheinlichkeit für ein Ansprechen auf die Therapie haben. Aus Gründen der Wirtschaftlichkeit, der Praktikabilität und des Nutzens ist eine Stratifizierung bis zu „Gruppen“, die nur noch von Einzelpersonen besetzt sind, nicht möglich. Der Terminus „Personalisierte Medizin" darf nicht dahingehend (miss)verstanden werden, dass jede einzelne Person ihr individuelles Arzneimittel - bildhaft gesprochen einen maßgeschneiderten Anzug - bekommt. Vielmehr bedeutet der Terminus, dass die stratifizierten Personengruppen entsprechend ihren gemeinsamen (z.B. genetischen) Merkmalen optimiert behandelt werden - bildhaft gesprochen den Anzug in verschiedenen Größen angeboten bekommen, aber nicht maßgeschneidert.

Bisher befindet sich die biomarkerbasierte Stratifizierung noch in einem frühen Entwicklungsstadium, so dass aktuell erst wenige Produkte Marktreife erlangt haben. Der Nutzen der Biomarkerforschung liegt derzeit vor allem im grundlegenden Erkenntnisgewinn über die den jeweiligen Krankheiten zugrunde liegenden biologischen und molekularen Prozesse, der Bereitstellung von Ressourcen, Methoden und neuen konzeptionellen Ansätzen und die Generierung von Hypothesen für weiterführende Forschungsarbeiten zur Steigerung der Effizienz der präklinischen und klinischen Forschung in der Pharmaindustrie.

Zielsetzung der Personalisierten Medizin ist es, die Gesundheitsversorgung mit Arzneimitteln auf längere Sicht qualitativ zu verbessern, sicherer und effizienter zu machen. Basierend auf neuen Erkenntnissen über die molekularen Mechanismen der Krankheitsentstehung werden: 
- Die Wirksamkeit von Arzneimitteln durch entsprechende diagnostische Tests sichergestellt.

- Die Dosierung von Arzneimitteln optimiert.

- Die Sicherheit der Arzneimitteltherapie erheblich erhöht und potentielle Nebenwirkungen bereits vor Therapiebeginn abgeschätzt und entsprechende Vorkehrungen getroffen.

- Unwirksame Therapien und damit einhergehende Kosten vermieden.

- Erkrankungsrisiken abschätzbar, so dass präventive Maßnahmen eingeleitet bzw. Krankheiten rechtzeitig behandelt werden können.

Neue und auf Patientengruppen zugeschnittene Arzneimittel sind heute schon Realität. Insofern ist die Personalisierte Medizin mehr eine Evolution als eine Revolution. In Tabelle 1 sind diejenigen Arzneimittel aufgelistet, bei denen bereits heute vor der Anwendung ein Gentest (oder ein Test, der den Genstatus indirekt ermittelt) vorgeschrieben oder empfohlen wird. Die Auflistung bezieht sich auf Deutschland und basiert auf öffentlichen Bekanntmachungen wie z.B. dem öffentlichen Bewertungsbericht der Europäischen Zulassungsagentur, EMA.

Der zukünftige Erfolg wird entscheidend davon abhängen, die Kompetenzen in Diagnostik, Pharmazie und Biotechnologie innerhalb oder zwischen Unternehmen und Forschungseinrichtungen entsprechend zu verzahnen und die Prozesse entlang des Wertschöpfungsprozesses so aufzustellen, dass personalisierte Therapiestrategien möglich sind. Die Komplexität ist vor allem dadurch bedingt, dass Zeitabläufe, Zulassungsvoraussetzungen, benötigte Verfahren und Systeme, Patentschutz, Konzepte für Marktausbietung und Erstattung und das unterschiedliche Know-how bereits im F\&E-Stadium synchronisiert werden müssen. Das ist nicht nur eine organisatorische und finanzielle Herausforderung im Hinblick auf die Strukturen und den assoziierten Investitionsbedarf, sondern für Roche eine fundamentale Frage für den Erfolg des Geschäftsmodells.

Die Personalisierte Medizin schafft einen Nutzen für alle Beteiligten im Gesundheitswesen vor allem für Patienten - und kann dadurch auch den ökonomischen Erfolg innovativer Pharmaunternehmen steigern. Der wirtschaftliche Erfolg der vermeintlichen „Marktreduktion“ infolge der therapeutischen Stratifizierung liegt in den Chancen für alle Beteiligten. 
- Für Patienten erhöhen sich die Erfolgsaussichten und der Erfolg einer Therapie signifikant, bei gleichzeitig sinkenden Nebenwirkungen.

- Ärzte können mithilfe von diagnostischen Tests diejenigen Patienten identifizieren, die für eine Therapie in Frage kommen. Damit lassen sich Indikationsstellung und Therapieauswahl optimieren. Anhand spezifischer Biomarker können Vorhersagen zu den Erfolgsaussichten einer Therapie getroffen werden - bereits vor Beginn der Therapie und durch Monitoring während der laufenden Therapie. Das erhöht die Behandlungssicherheit klinisch signifikant.

- Für Kostenträger liegen die Vorteile der Personalisierten Medizin in erster Linie in der Erhöhung der Kosteneffizienz. Unnötige Kosten aufgrund von vermeidbaren Nebenwirkungen oder einem Nicht-Ansprechen auf das Arzneimittel werden vermieden. Die Steigerung der Kosteneffizienz liegt auch im Interesse einer auf Kostendämpfung orientierten Gesundheitspolitik.

- Die Erhöhung der Behandlungssicherheit und Sicherstellung eines Behandlungserfolges wird zum entscheidenden Argument in der Diskussion bei der Marktzulassung und Erstattung durch die Kostenträger.

Aus Sicht des Pharmaunternehmens bietet die Personalisierte Medizin jedoch noch weitere Vorteile: Durch die Fokussierung auf definierte Patientengruppen lassen sich die Ausgaben für Marketing und Vertrieb senken. Effektivitäts- und Effizienzsteigerungen in F\&E sind ebenfalls möglich. Klinische Studien sind ein Kostentreiber in der Pharmaentwicklung. Bis dato werden überwiegend in unspezifischen, populationsbasierten klinischen Prüfungen große Patientenzahlen rekrutiert, um Behandlungserfolge nachzuweisen. Die Personalisierte Medizin wird zukünftig klinische Prüfungen an kleineren, therapeutisch stratifizierten Patientenpopulationen ermöglichen. An den Studien nehmen selektiv nur noch solche Patienten teil, die aufgrund von Biomarkern bzw. einem genetischen Profil für die Behandlung geeignet sind. Damit können die Anzahl der Patienten und die Dauer der klinischen Prüfung reduziert und so potentiell Kosten gespart werden. Die Personalisierte Medizin ist auch ein strategischer Vorteil, um sich im Wettbewerb zu differenzieren. Die Kombination von Diagnostikum und Therapeutikum ist in Entwicklung und Vermarktung für viele Hersteller perspektivisch zu komplex.

Die Rahmenbedingungen für einen zielgerichteteren Ansatz in der Medizin sind in Deutschland noch nicht optimal. So sind z.B. die Zeitabläufe, die Zulassungsvoraussetzungen, die Bewertungs- und Erstattungsverfahren für Arznei- 
mittel und Diagnostika nicht aufeinander abgestimmt: Mit der Markteinführung des Brustkrebsmedikamentes Herceptin ${ }^{\circledR}$ stellte Roche den diagnostischen HER2-Test kostenlos über Jahre zur Verfügung. Damit sollte sichergestellt werden, dass nur die Patientinnen mit dem Medikament behandelt werden, die auch davon profitieren können. Der Test ist erst mehr als zwei Jahre nach Markteinführung in die Erstattung aufgenommen worden. Dies zeigt, dass die Inkongruenz unserer Zulassungs- und Erstattungssysteme unnötige Kosten verursacht, im schlimmsten Fall Patienten schadet, statt Nutzen und Effizienz aus der neuen Technologie zu ziehen.

Die Personalisierte Medizin ist u.a. auf genetische Untersuchungen angewiesen - dafür müssen entsprechende gesellschaftliche Voraussetzungen geschaffen werden. Die gesellschaftliche Akzeptanz ist ein wichtiges Kriterium, d.h. genetische Untersuchungen dürfen nicht automatisch negative Assoziationen hervorrufen.

Eine unabdingbare Voraussetzung ist auch der ausreichende Patentschutz für Innovationen. Damit Unternehmen F\&E-Investitionen tätigen und innovative Therapiekonzepte Realität werden, benötigen sie verlässliche und zukunftsfähige Rahmenbedingungen für den Schutz ihres geistigen Eigentums. Dies rechnet sich auch für das „System“. Die Innovationen von Heute sind die Billigmedizin von Morgen. Wer die schon heute existierenden Möglichkeiten und die zukünftigen Chancen, die sich durch Personalisierte Medizin eröffnen, klar kommuniziert, wird auf ein zunehmend größeres Verständnis und eine höhere Akzeptanz für dieses innovative Therapiekonzept stoßen.

Wenn die Chancen, die sich mit der Personalisierten Medizin bieten, genutzt werden, dann geht der Trend innerhalb der Pharmaunternehmen weg von dem „one size fits all“ Modell, so dass zukünftig nicht mehr die hohe Anzahl initial behandelter Patienten entscheidend sein wird. Vielmehr wird durch die gezielte therapeutische Stratifizierung die Anzahl initial behandelter Patienten geringer, dafür aber die Anzahl nachhaltig erfolgreich therapierter Patienten größer.

Für die Pharmaunternehmen bietet die vordergründige „Marktreduktion“ eine Chance, wenn der klinische Nutzen stärker zunimmt als die Populationsgröße sinkt. Das heißt aber auch im Umkehrschluss, dass die Kosten für den Einzelfall möglicherweise steigen werden - bei gleichzeitiger Zunahme der SystemEffizienz (weniger Fehlbehandlungen). Neu wäre die Chance für die Industrie, 
die Ärzteschaft und die Kassen, Therapie-Angebote zu entwickeln die letztlich nicht nur den Einsatz von Tests und Arzneimitteln bezahlen, sondern den nachgewiesenen Nutzen für den Patienten.

Vier Punkte könnten die Erfolgsaussichten der Personalisierten Medizin steigern:

- Die Pharma- und Diagnostika-Industrie verpflichtet sich, gemeinsam validierte Testverfahren und Biomarker zu entwickeln.

- Die Aus- und Weiterbildung der Ärzteschaft im Bereich der Therapie bestimmenden oder Therapie begleitenden Diagnostik muss verbessert werden.

- Die aktuellen Erkenntnisse der Personalisierten Medizin sollten in die entsprechenden Behandlungsleitlinien aufgenommen werden.

- Die Zulassungs- und Erstattungsvoraussetzungen medizinisch notwendiger und wirtschaftlicher diagnostischer Testung müssen angepasst werden.

In Deutschland sind die Weichen für die Zukunft der Personalisierten Medizin schon gestellt: Auf Initiative des deutschen Gesundheitsministerium hat sich eine aus den verschiedenen Interessengruppen gebildete Task Force „Pharma“ der patientenorientierten Zukunftstechnologie ,Individualisierte Arzneimitteltherapie" gewidmet. Sie legt in ihrem dritten Bericht eine detaillierte Situationsanalyse und daraus abgeleitete Maßnahmen zur Förderung dieser Zukunftstechnologie vor. Aufgabe der Steuerungsgruppe war es mögliche Hemmnisse im Bereich Zulassung und Erstattung dieser Produkte zu identifizieren und Handlungsempfehlungen mit dem Ziel zu erarbeiten, den Herstellern einen schnellen Marktzugang zu ermöglichen.

Die Personalisierte Medizin ist die konsequente Weiterentwicklung des Geschäftsmodells innovativer und forschender pharmazeutischer Unternehmen und der Schlüssel für eine effektivere und effizientere Arzneimittelversorgung auch wenn es bis dahin noch ein langer und steiniger Weg ist. Wir legen heute den Grundstein für die Medizin, die uns in 2020 zur Verfügung stehen wird. 
Tabelle 1: Wir stehen erst am Anfang - Beispiele für Produkte am Markt

\begin{tabular}{|c|c|c|c|c|c|}
\hline \multicolumn{2}{|c|}{ Onkologie } & \multicolumn{2}{|c|}{ Virologie/ Infektionskrankheiten } & \multicolumn{2}{|c|}{ ZNS Erkrankungen } \\
\hline Tamoxifen & ERIPR Status & Pegasys $/$ Copegus ${ }^{*}$ & $\begin{array}{l}\text { HCV Genotypi- } \\
\text { sierung }\end{array}$ & Phenytoin & CYP2C9 \\
\hline Tamoxifen & BRCA1 & $\begin{array}{l}\text { HIV Prot. Inh. } \\
\left(1^{*} \text { ta mikt) }\right.\end{array}$ & Viruslast & Venlafaxin & CYP2D6 \\
\hline Tamoxifen & BRCA2 & $\begin{array}{l}\text { HIV Prot. Inh } \\
\left(2^{\text {nd }} \text { to mkt }\right)\end{array}$ & Viruslast & Modafinil & CYP2D6 \\
\hline Tamoxifen & CYP2D6 & $\begin{array}{l}\text { HIV Prot Inh. } \\
\left(1{ }^{*} \text { to mkt }\right)\end{array}$ & $\begin{array}{l}\text { HIV Genotypis- } \\
\text { ierung }\end{array}$ & Resperidon & CYP2D6 \\
\hline Chemotherapie & Oncotype Dx & $\begin{array}{l}\text { HIV Prot Inh } \\
\left(2^{2 d} \text { to mkt }\right)\end{array}$ & $\begin{array}{l}\text { HIV Genotypis- } \\
\text { ierung }\end{array}$ & Atomoxetin & CYP2DG \\
\hline Arimidex ${ }^{8}$ & ER/PR Status & Isoniazid & NAT & Thioridazin & CYP2D6 \\
\hline Herceptin & HER2 Assay & Tamiflus & $\begin{array}{l}\text { Influenza A/B } \\
\text { Test }\end{array}$ & Levodopa & COMT \\
\hline Xeloda" & Enzymaktivität & PegintronA $A^{\%}$ & HCVEVL & Tasmar & COMT \\
\hline $\begin{array}{l}\text { 6- } \\
\text { Mercaptopurin }\end{array}$ & TPMT & Roferon & HCVEVL & Aripiprazol & CYP2D6 \\
\hline Glivec $^{*}$ & BCR-ABL & & & & \\
\hline Glivec & C-Kit & \multirow{2}{*}{\multicolumn{2}{|c|}{ Atemwegserkrankungen }} & \multirow{2}{*}{\multicolumn{2}{|c|}{$\begin{array}{l}\text { Metabolische und vasku- } \\
\text { läre Erkrankungen }\end{array}$}} \\
\hline Dasatinib & BCR-ABL & & & & \\
\hline Iressa" & EGFR Status & Prolastin & $\mathrm{PIZZ}, \mathrm{PiZ} \mathrm{Pi}$ & Fosamax & PINP \\
\hline Tarceva" & EGFR/HER1 & \multirow{3}{*}{\multicolumn{2}{|c|}{ Theophyllin }} & Somatropin & Chr 15 \\
\hline Irinotecan & UGT1A1 & & & Insulin & HbATC \\
\hline Erbitux & $\begin{array}{l}\text { EGFR Status, } \\
\text { KRAS Wt }\end{array}$ & & & Simvastatin & Lipidprofile \\
\hline Retinoinsäure & $\begin{array}{l}\text { PML/RAR } \\
\text { Gen }\end{array}$ & \multicolumn{2}{|c|}{ Gastrointestinalerkrankungen } & \multicolumn{2}{|c|}{$\begin{array}{c}\text { Halmatologische Erkran- } \\
\text { kungen }\end{array}$} \\
\hline MabThera & $\mathrm{CD} 20$ & Ompeprazol & CYP2C19 & Warfarin & CYP2C9 \\
\hline Tykerb & EGFR Status & \multirow{2}{*}{$\begin{array}{l}\text { PPI } \\
\text { und Antibiotika }\end{array}$} & \multirow[t]{2}{*}{ HPylori } & Warfarin & VKORC1 \\
\hline Vectibix & K-ras Status & & & Heparin & APTT \\
\hline & & & & EPO & $\mathrm{CBC}$ \\
\hline \multicolumn{2}{|c|}{$\begin{array}{l}\text { Autoimmunerkrankungen/ } \\
\text { Transplantation }\end{array}$} & \multicolumn{2}{|c|}{ Kardiovaskuläre Erkrankungen } & \multicolumn{2}{|c|}{ Anăsthesie } \\
\hline CellCeptis & IA & BiDiL & Ethnizităt & Succinylcholin & $\begin{array}{l}\text { Pseudocholin- } \\
\text { esterasewerte }\end{array}$ \\
\hline Azathioprin & TPMT & Hydralazin & NAT & & \\
\hline $\begin{array}{l}\text { Neoralsionc } \\
\text { losporin) }\end{array}$ & IA's fur CsA & Procainamid & NAT & & \\
\hline $\begin{array}{l}\text { Prograf (Ta- } \\
\text { crolimus) }\end{array}$ & IA & GPib/llia & Troponin & & \\
\hline $\begin{array}{l}\text { Rapamune } \\
\text { (Sirolimus) }\end{array}$ & IA & Streptokinase & Troponin & & \\
\hline MabThera" & RA Profile & & & & \\
\hline
\end{tabular}




\section{Literaturverzeichnis}

DiMasi, J./Grabowski, G.: The Cost of Biopharmaceutical R\&D: Is Biotech Different? Manage. Decis. Econ. 28/2007, S. $469 \mathrm{ff}$.

Flaherty, K. u.a.: Phase I study of PLX4032: Proof of concept for V600E BRAF mutation as a therapeutic target in human cancer. J Clin Oncol 27:15s, 2009 (suppl; abstr 9000).

Hüsing, B. u.a.: Individualisierte Medizin und Gesundheitssystem. Zukunftsreport. Juni 2008. Abschlussbericht Nr. 126. Hrsg. TAB - Büro für Technikfolgen-Abschätzung beim Deutschen Bundestag.

http://www.tab.fzk.de

Suchbegriff: „Publikationen“, (Abgerufen 9.07.2009).

Lazarou, J. u.a.: Incidence of adverse drug reactions in hospitalized patients: a meta-analysis of prospective studies. JAMA 1998;279(15):1200-5.

Price Waterhouse Coopers Report: Pharma 2020: The Vision. Which Path Will You Take? 2009, S. 5.

www.pwc.com

Suchbegriff: "Pharma 2020", (Abgerufen: 9.07.2009).

Spear, BB. u.a.: Clinical application of pharmacogenetics. Trends Mol Med. 2001; 7(5):201-204.

Süddeutsche Zeitung. „Medikamente und Nebenwirkungen. Bis zu 25.000 Todesfälle durch Medikamente". 9.11.2007

www.sueddeutsche.de,

Suchbegriff: „25.000 Tote“, (Abgerufen 9.07.2009).

$V F A$ - Verband Forschender Arzneimittelhersteller. „In Deutschland zugelassene Arzneimittel für die individualisierte Medizin.“

www.vfa.de,

Suchbegriff: „Individualisierte Medizin“, (Abgerufen 9.07.2009). 
Eberhard Wille and Klaus Knabner - 978-3-631-75550-1 Downloaded from PubFactory at 01/11/2019 03:39:43AM via free access 
Verfahren zur Frühbewertung des Nutzens neuer, patentgeschützter Arzneimittel - Sicht der Arzneimittelkommission der deutschen Ärzteschaft

\section{Wolf-Dieter Ludwig}

\section{Einleitung}

„Where will new drugs come from?" und "Why there is still an innovation deficit?", diese in einem Editorial im Lancet kürzlich gestellten Fragen kennzeichnen den im letzten Jahrzehnt zu beobachtenden Trend in der Entwicklung neuer Arzneimittel und verdeutlichen gleichzeitig, dass trotz großer Fortschritte in der molekularbiologischen Grundlagenforschung und Biotechnologie nur sehr wenige der neu zugelassenen Wirkstoffe einen therapeutisch relevanter Fortschritt bedeuten (Anonym, Lancet 2011). Zu den vielfältigen Gründen für diesen Trend gehören (a) die deutliche Abnahme in der Produktivität pharmazeutischer Unternehmen (PU) bei der Entwicklung neuer Wirkstoffe, (b) das Scheitern viel versprechender und in der Entwicklung teurer neuer Biopharmazeutika vor der Zulassung aufgrund unzureichender Wirksamkeit, (c) der „Untergang der Blockbuster“ (sog. „Kassenschlager-Arzneimittel“ mit Jahresumsatz weltweit $>1$ Mrd. US-Dollar) infolge auslaufender Patente in den Jahren 2008-2012 und (d) die Marktrücknahme von Wirkstoffen (z. B. Rofecoxib, Rosiglitazon) mit hohen Umsätzen und häufiger Verordnung infolge schwerwiegender unerwünschter Arzneimittelwirkungen (UAW) (Honig 2011; Kaitin 2011).

Global agierende PU haben sich deshalb für eine strategische Neuausrichtung im Bereich Forschung und Entwicklung neuer Arzneimittel entschieden und aus ökonomischen Gründen, aber auch angesichts der demographischen Entwicklung, ihre Forschungsaktivitäten auf Bereiche konzentriert, in denen die Patientenbedürfnisse noch nicht durch existierende medikamentöse Therapien abgedeckt (,unmet medical need“) und deshalb profitable Märkte (Bsp. onkologische und rheumatologische Erkrankungen, Demenz, multiple Sklerose, HIVInfektionen) vorhanden sind. Eine in den letzten Jahren von PU verfolgte Alternativstrategie, die Entwicklung und Zulassung von Arzneimitteln („Orphan Drugs“, OD) für seltene Erkrankungen (sog. „Nichebuster“), hat sich als ausgesprochen erfolgreich erwiesen, da bei einigen dieser OD nach Marktzugang rasch eine Ausweitung ihrer Anwendungsgebiete erreicht werden konnte und aus „Nichebuster“ somit „Blockbuster“ wurden (Owens 2007; Hughes et al. 2008). Leider steht hierbei eine bedarfs- und patientenorientierte Arzneimittel- 
entwicklung häufig nicht im Mittelpunkt, sondern es werden in erster Linie Gewinninteressen durchgesetzt. Anstatt auch durch öffentliche Mittel die Entwicklung neuer Arzneimittel adäquat zu honorieren, deren bessere Wirksamkeit oder Verträglichkeit in klinischen Studien überzeugend belegt werden konnte, werden derzeit bei der Zulassung den PU eher Anreize für die Entwicklung von neuen Wirkstoffen mit in der Regel geringem oder nicht nachgewiesenem $\mathrm{Zu}$ satznutzen geboten, die nach Zulassung dann bei häufig überhöhten Preisen um ihren Marktanteil mit anderen Arzneimitteln konkurrieren (Light 2011).

Die Ausgaben der gesetzlichen Krankenversicherung (GKV) für die im ambulanten Bereich verordneten Arzneimittel in Deutschland sind in den letzten Jahren stark gestiegen und liegen seit dem Jahr 2001 über den Ausgaben für ärztliche Behandlung. Im Jahr 2009 wurden in der GKV für Arzneimittel insgesamt 32,4 Mrd. $€$ (ohne Impfstoffkosten) ausgegeben. Dies entspricht einem Anstieg von 1,5 Mrd. $€(4,8 \%)$ gegenüber 2008 und der Anteil der Arzneimittelausgaben an den Gesamtausgaben der GKV beträgt inzwischen $18 \%$. Dieser Trend setzt sich seit Jahren fort. Verursacht wird der Ausgabenanstieg in erster Linie durch höhere Ausgaben für Arzneimittel ohne Festbetrag (Anstieg in 2009 um 8,9\%) sowie kostenintensive Spezialpräparate mit jährlich zweistelligen Wachstumsraten (Schwabe 2010). Spezialpräparate werden in steigendem Umfang in der Onkologie, dort häufiger auch als OD (siehe 3.), in der Transplantationsmedizin, bei chronischen entzündlichen Erkrankungen, bei HIV-Patienten und bei seltenen Krankheiten wie pulmonaler Hypertonie und lysosomalen Speicherkrankheiten eingesetzt. Die Ausgaben für Spezialpräparate sind von 2,5 Mrd. Euro in Jahr 2000 auf 7,8 Mrd. $€$ im Jahr 2009 gestiegen und inzwischen verantwortlich für 27,5\% der Arzneimittelausgaben in der GKV, obwohl Spezialpräparate nur $2,5 \%$ der Verordnungen ausmachen. Spezialpräparate sind in Deutschland verantwortlich für mehr als 50\% des Anstiegs der Arzneimittelausgaben seit 2000. Unter den 10 umsatzstärksten patentgeschützten Arzneimittel befanden sich in Deutschland im Jahr 20095 Spezialpräparate (Adalimumab, Etanercept, Interferon beta-1a, Imatinib, Glatiramer), deren Preise in Deutschland durchweg deutlich höher lagen als beispielsweise in den meisten anderen Mitgliedsstaaten der Europäischen Union (EU) (Schwabe 2010). Die im europäischen Vergleich sehr hohen Arzneimittelausgaben in Deutschland und die Gründe für die Unterschiede bei den Arzneimittelpreisen und beim Zugang zu neuen Arzneimitteln wurden ausführlich in einer Studie analysiert, die der EU-Gesundheitsausschuss des Europäischen Parlaments 2010 in Auftrag gegeben hatte (Policy Department A: Economic and Scientific Policy 2011). Neben dem Bruttoinlandsprodukt (BIP) 
sowie demographischen und nationalen gesundheitspolitischen Faktoren sind insbesondere unterschiedliche Regulierungsansätze für die z.T. enorme Preisdifferenz für Arzneimittel zwischen den EU-Mitgliedsstaaten verantwortlich. Angesichts der rasch steigenden Ausgaben für Arzneimittel haben zahlreiche Länder in der EU bereits seit einigen Jahren unterschiedliche Maßnahmen ergriffen (Übersichten z.B. bei Sermet 2010 und Zentner 2011), um Arzneimittelausgaben zu senken, ohne dabei den Zugang zu echten Innovationen einzuschränken und die Qualität der Arzneimitteltherapie zu verschlechtern. Deutschland war bis 2011, mit Ausnahme von Dänemark und Malta, in Europa der einzige Arzneimittelmarkt ohne direkte Preisregulierung für patentgeschützte Arzneimittel und diente gleichzeitig als Referenzland für Arzneimittelpreise in mehreren europäischen Staaten. Die hohen Arzneimittelpreise in Deutschland haben auch dazu geführt, dass PU bestrebt waren, neue Arzneimittel zuerst in Deutschland auf den Markt zu bringen, damit dieser hohe Preis als Referenzpreis auch Eingang in die internationalen Preisvergleiche anderer Länder findet.

Angesichts der vor allem für Spezialpräparate rapide gestiegenen Arzneimittelkosten bestand deshalb im Jahr 2010 für das Bundesministerium für Gesundheit (BMG) kurzfristiger Handlungsbedarf, dem die Fraktionen der CDU/CSU und FDP mit dem Gesetz zur Neuordnung des Arzneimittelmarktes in der GKV (AMNOG) nachgekommen sind. Das AMNOG, aus Sicht des BMG ein „Dreiklang aus strukturellen Veränderungen, dem Abbau von Überregulierung und kurzfristigen Einsparungen", sieht nun zusammen mit einer bereits am 30. Juli 2010 in Kraft getretenen Regelung im Gesetz zur Änderung krankenversicherungsrechtlicher und anderer Vorschriften (GKV-ÄndG) Einsparungen in Höhe von 2,4 Mrd. $€$ pro Jahr vor, davon 2,2 Mrd. $€$ für die GKV (BMG 2010). Die kurzfristig angestrebten Einsparungen bei den Arzneimittelkosten ab dem Jahr 2011 umfassen verschiedene Maßnahmen. Hierzu zählen u. a. die Erhöhung der gesetzlichen Hersteller-Rabatte für Arzneimittel ohne Festbetrag von $6 \%$ auf 16 $\%$ vom 01. August 2010 bis zum 31. Dezember 2013 einschließlich Preismoratorium, die Anhebung des Apothekenrabatts von 1,75€ auf 2,05€ je Packung verschreibungspflichtiger Arzneimittel in den Jahren 2011 und 2012 (nur GKV), eine Absenkung des Volumens des Großhandelzuschlags für rezeptpflichtige Arzneimittel (GKV und private Krankenversicherung, PKV) und die Übertragung der gesetzlichen Hersteller-Rabatte auf die PKV.

Dieser Beitrag verdeutlicht die Position der Arzneimittelkommission der deutschen Ärzteschaft (AkdÄ) zu den vom BMG als „strukturelle, langfristig 
wirksame Veränderungen“" apostrophierten Reformen im AMNOG. Stellungnahmen der Bundesärztekammer, erstellt in Zusammenarbeit mit der AkdÄ, zum Referentenentwurf für das AMNOG und zum Entwurf für die ArzneimittelNutzenbewertungsverordnung können über die Homepage der AkdÄ abgerufen werden (www.akdae.de/Stellungnahmen/BMG/20101125.pdf; www.akdae.de/ Stellungnahmen/BMG/20100809.pdf).

In diesem Beitrag wird insbesondere eingegangen auf Änderungen des $\S 35 \mathrm{a}$, $\S 92, \S 130$ des Sozialgesetzbuches (SGB) Fünftes Buch (V) sowie eine Änderung des $\S 42 \mathrm{~b}$ im Arzneimittelgesetz (AMG) und weitere, im AMNOG nicht umgesetzte Maßnahmen, die für eine optimierte Einführung neuer Arzneimittel in die Versorgung und evidenzbasierte Nutzenbewertung jedoch erforderlich sind.

Eine detaillierte Darstellung der Frühbewertung des Nutzens neu zugelassener Arzneimittel und der Entscheidungsgrundlagen hierfür aus Sicht des Spitzenverbandes der GKV und des Gemeinsamen Bundesausschusses (GBA) finden sich auch in diesem Band der Bad Orber Gespräche (v. Stackelberg 2011) bzw. sind kürzlich publiziert worden (Hess 2011). Angesichts der in der Öffentlichkeit herrschenden Verwirrung und z.T. auch nicht korrekten Verwendung der Begriffe „Wirksamkeit-Risiko-Verhältnis“, „Nutzen“ und „Zusatznutzen“ von neuen Arzneimitteln wird zu Beginn kurz auf die unterschiedlichen Anforderungen an Arzneimittel mit neuen Wirkstoffen bzw. Anwendungsgebieten im Rahmen der Zulassung bzw. arzneimittelrechtlichen und sozialrechtlichen Nutzenbewertung eingegangen.

\section{Zulassung: Anforderungen und begrenzte Kenntnisse über Wirksam- keit und Risiken neuer Arzneimittel}

Im Rahmen der Zulassung werden Qualität, Wirksamkeit und medizinische Unbedenklichkeit eines neuen Wirkstoffs in dem vorgesehenen Anwendungsgebiet von den nationalen Zulassungsbehörden bzw. der European Medicines Agency (EMA) geprüft und bei Vorliegen eines positiven Nutzen-Risiko-Verhältnis (,benefit-risk-profile“) die Zulassung erteilt. Pharmakoökonomische Aspekte spielen bei den Zulassungsentscheidungen keine Rolle und auch die Identifizierung von Patientenuntergruppen (z.B. anhand von Biomarkern in der Onkologie), die von einem neuen Wirkstoff tatsächlich profitieren, ist nicht zwingende Voraussetzung für die Zulassung. Dabei sollten entsprechend der Richtlinie 
2001/83/EG zur Schaffung eines Gemeinschaftskodexes für Humanarzneimittel klinische Prüfungen in der Regel als „,kontrollierte klinische Prüfungen“ und soweit möglich randomisiert durchgeführt werden, wobei zum Vergleich je nach Einzelfall ein Placebo oder ein bereits bekanntes Arzneimittel mit nachgewiesenem therapeutischen Wert heranzuziehen ist. Untersuchungen in den letzten Jahren haben jedoch gezeigt, dass diese Vorgaben von der EMA im Rahmen der Zulassung neuer Arzneimittel nicht immer konsequent eingehalten wurden. So wurde in nur etwa $50 \%$ der in den europäischen öffentlichen Bewertungsberichten (European Public Assessment Report, EPAR) erwähnten klinischen Studien (Zeitraum der Auswertung: 1. Januar 2007 bis 31. Dezember 2008) der neue Wirkstoff mit einem Arzneimittel mit nachgewiesener therapeutischer Wirksamkeit (,active comparator") verglichen und nur in etwa $20 \%$ der Fälle war das Design der Zulassungsstudie - in der og. Richtlinie allerdings auch nicht gefordert - darauf ausgerichtet, eine Überlegenheit des neuen Wirkstoffs im Vergleich zum ,active comparator" in randomisierten kontrollierten Studien (RCT) zu belegen (Eichler et al. 2010). Dies hat zur Folge, dass in Zulassungsstudien beim Vergleich neuer mit bereits vorhandenen Arzneimitteln sehr häufig eine Gleichwertigkeit (,equivalence“) oder sogar nicht nachgewiesene Unterlegenheit (,non-inferiority“) des neuen Wirkstoffs als für die Wirksamkeit ausreichender Beleg akzeptiert wird. Weitere Merkmale, die die Übertragbarkeit der Ergebnisse aus Zulassungsstudien zu neuen Arzneimitteln auf die Behandlung von Patienten unter Alltagsbedingungen in Klinik oder Praxis (externe Validität) einschränken, sind die häufig strikten Ein- und Ausschlusskriterien, Verwendung von Surrogat- bzw. kombinierten Endpunkten als primäre Endpunkte, die unzureichende Berücksichtigung patientenrelevanter Parameter (z.B. „patient reported outcomes" wie Lebensqualität und Symptomkontrolle) und die in $\mathrm{Zu}$ lassungsstudien häufig sehr kurzen Zeiträume der Behandlung bzw. Nachbeobachtung. Aussagen zur Sicherheit bzw. zu den unerwünschten Arzneimittelwirkungen (UAW) neuer Wirkstoffe sind in Zulassungsstudien nur sehr eingeschränkt möglich, da aufgrund der häufig kleinen Patientenzahlen und in der Regel kurzen Studiendauer bzw. Nachbeobachtung fast ausschließlich (sehr) häufige bzw. akut auftretende UAWs erfasst werden. Mit der Zulassung neuer Wirkstoffe sind deshalb heute häufig im Rahmen der Pharmakovigilanz (z.B. Pläne zum Risikomanagement nach Zulassung) Bedingungen verknüpft, die eine sichere und wirksame Verordnung bzw. Anwendung gewährleisten sollen. Darüber hinaus werden zunehmend weitere Untersuchungen zur Sicherheit gefordert, die aber nicht immer rechtzeitig durchgeführt werden (Okie 2010). Aktuel- 
le Ergebnisse zu den erst nach Zulassung beobachteten UAWs von Biopharmazeutika, die bei fast einem Drittel der neuen Wirkstoffe zu regulatorischen Maßnahmen (z.B. Warnhinweise, Veränderungen der Fachinformation bzw. Packungsbeilage oder sogar Marktrücknahmen) führten, zeigen wie notwendig derartige Studien sind (Giezen 2008).

Mit der Zulassung wird einem neuen Arzneimittel ein positives „Nutzen (besser Wirksamkeit)-Risiko-Verhältnis“" attestiert, obwohl zu diesem Zeitpunkt nur begrenzt Ergebnisse aus klinischen Studien zu Wirksamkeit sowie Sicherheit zur Verfügung stehen und Vergleiche zur medikamentösen Standardtherapie bzw. zu nicht-medikamentösen Therapiestrategien, die für eine Bewertung des Nutzens bzw. Zusatznutzens des neuen Wirkstoffs benötigt werden, selten vorliegen. Zielsetzungen von AMG und SGB V sind deshalb auch unterschiedlich. Anders als bei der Bewertung der Wirksamkeit eines neuen Arzneimittels unter den eher den Idealfall als die Alltagspraxis widerspiegelnden Bedingungen einer klinischen Zulassungsstudie stehen bei der sozialrechtlichen Bewertung Aspekte der therapeutisch und wirtschaftlich effizienten Versorgung von Patienten im Vordergrund. Entsprechend $\S 35 \mathrm{~b}$ im SGB V sind beim Patientennutzen insbesondere die Verbesserung des Gesundheitszustandes, eine Verkürzung der Krankheitsdauer, eine Verlängerung der Lebensdauer, eine Verringerung der Nebenwirkungen sowie eine Verbesserung der Lebensqualität zu berücksichtigen. Dabei beruht die Nutzenbewertung auf einer vor der Zulassung meistens nicht möglichen Abwägung zwischen positiven (Wirksamkeit) und negativen (Risiken bzw. Schäden), patientenrelevanten therapeutischen Effekten unter Alltagsbedingungen im Hinblick auf die Anwendungsgebiete des neuen Wirkstoffs. Sie sollte stets anhand von Vergleichen gegen den geltenden medizinischen Standard (,zweckmäßige Vergleichstherapie“) erfolgen.

Zahlreiche Beispiele aus den letzen Jahren belegen, dass neue Wirkstoffe (z.B. Anagrelid, Bevacizumab, Ezetimib/Simvastatin, Olanzapin, Oseltamivir), deren Wirksamkeit in den Zulassungsstudien gezeigt wurde und die nach Zulassung weltweit mitunter Umsätze von $>1 \mathrm{Mrd}$. US-Dollar/Jahr erzielten, beim Vergleich mit den verfügbaren medikamentösen Therapiealternativen keinen Zusatznutzen im Sinne einer besseren Wirksamkeit oder geringeren Toxizität aufwiesen. 


\section{Frühbewertung des (Zusatz-)Nutzens von Arzneimitteln mit neuen Wirkstoffen}

Im Mittelpunkt des AMNOG steht die sogenannte frühe Nutzenbewertung von Arzneimitteln mit neuen Wirkstoffen bzw. neuen Anwendungsgebieten, die vom GBA vorgenommen wird (Hess 2011). Der neue § 35a des SGB V sieht vor, dass zur Nutzenbewertung insbesondere die Bewertung des Zusatznutzens gegenüber der zweckmäßigen Vergleichstherapie, des Ausmaßes des Zusatznutzens und seiner therapeutischen Bedeutung gehört. Der Begriff des Zusatznutzens ist neu im SGB V und nicht gesetzlich definiert. Auch auf europäischer Ebene existiert keine allgemeingültige Definition. Verbraucherorganisationen in der EU sprechen von einem Zusatznutzen, wenn zuverlässige Ergebnisse klinischer Prüfungen vorliegen, die für Patienten eine bessere Wirksamkeit und/oder größere Sicherheit und/oder einfachere Verabreichung neuer Arzneimittel im Vergleich zu den vorhandenen Alternativen zeigen (Eichler 2010). Grundlage der Nutzenbewertung sind Nachweise des PU, die er als Dossier, einschließlich aller von ihm durchgeführten oder in Auftrag gegebenen klinischen Prüfungen, spätestens zum Zeitpunkt des erstmaligen in den Verkehr bringen bzw. der Zulassung neuer Anwendungsgebiete des Arzneimittels an den GBA elektronisch zu übermitteln hat. Die Angaben, die dieses Dossier enthalten muss, sind insbesondere: zugelassene Anwendungsgebiete, medizinischer Nutzen bzw. Zusatznutzen im Verhältnis zur zweckmäßigen Vergleichstherapie und Kosten der Therapie für die GKV. Das im AMNOG vorgeschriebene, auf dem Dossier des PU und der frühen Nutzenbewertung durch den GBA basierende Vorgehen zur Festlegung des Festbetrags bei Arzneimitteln, die pharmakologischtherapeutisch vergleichbar mit Festbetragsarzneimitteln sind, und der Erstattungsbeträge bei neuen Arzneimitteln ohne bzw. mit Zusatznutzen ist in Abbildung 1 (Anonym, AMB 2010) skizziert.

Auch für bereits zugelassene und im Verkehr befindliche Arzneimittel kann der G-BA gemäß $§ 35 a$ Absatz 6 des SGB V eine Bewertung veranlassen. Vorrangig sind Arzneimittel zu bewerten, die für die Versorgung von Bedeutung sind oder mit Arzneimitteln mit neuen Wirkstoffen im Wettbewerb stehen, für die ein Beschluss des GBA hinsichtlich der Bewertung des Zusatznutzens vorliegt. 


\section{Abbildung 1: Gesetz zur Neuordnung des Arzneimittelmarkts (AMNOG), Nut- zenbewertung von Arzneimitteln und Festsetzung der Erstat- tungsbeträge}

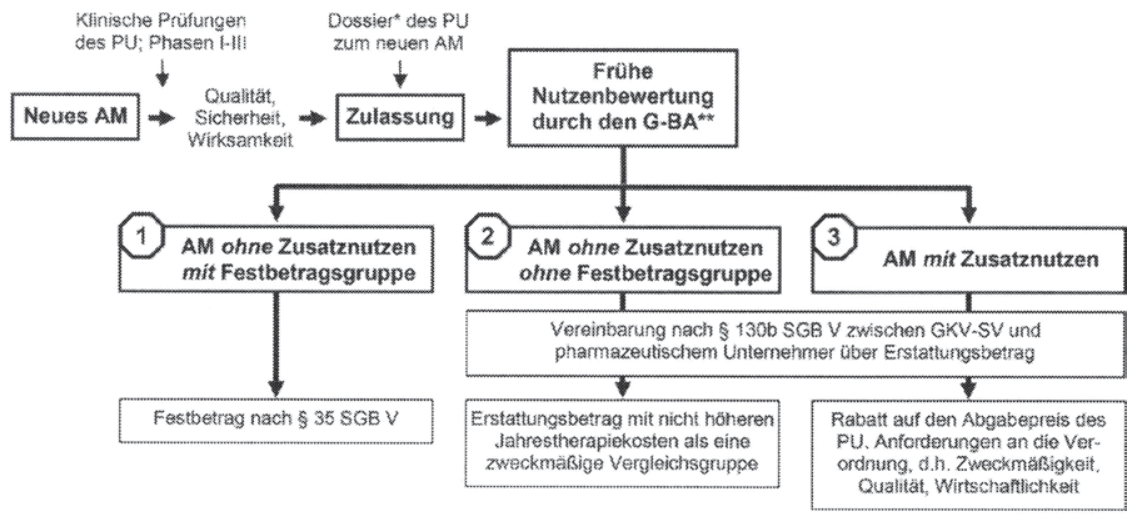

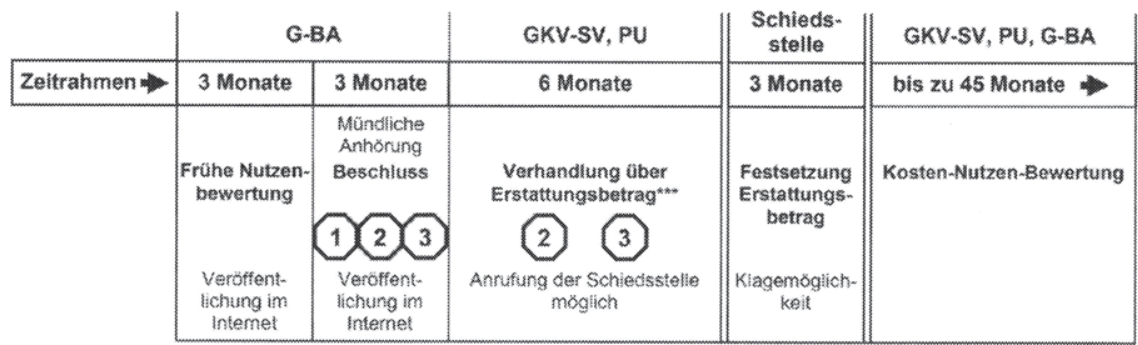

\footnotetext{
" - Das Dossier ist spätestens zum Zeitpunkt des inverkehrtringens bzw. der Zulassung nevier Anwendungsgebiete des AM an den G-BA ejektronisch zu übermitteln.

** = Die frühe Nutzenbewertung gilt auch für die Zulassung eines neuen Anwendungsgebietes. Nutzenbewertung kann der $G-B A$ auch für bereits zugelassene und in Verkehr befindiche AM: veranlassen (SGE V \$35a Absatz 6).

${ }^{* *}=$ Kommt eine Vereinbarung nicht innechalb von sechs Monaten nach Veroffentlichung des Beschlusses zustanda. entscheicet die zentrale Schiedsstelle.

$A M=$ Arzneimittel; $G-B A=$ Gemeinsamer Bundesatsschuss; GKV-SV = Gesetzliche Krankenversichenung-Spitzenverband; $\mathrm{PU}=$ Pharmazeutischer Unternehmer
}

\section{Arzneimittel für seltene Krankheiten: alternativlos und medizinischer Zusatznutzen belegt?}

Unter „orphan drugs“ (OD) versteht man Arzneimittel, die zur Behandlung seltener Krankheiten eingesetzt werden. Als selten gilt innerhalb der EU eine Krankheit, wenn sie nicht mehr als 5 pro 10.000 EU-Einwohner betrifft. Eine einheitliche Definition für den Begriff seltene Krankheit existiert jedoch nicht und die Häufigkeiten in Europa, den USA und Japan, bei denen von einer seltenen Krankheit gesprochen wird, unterscheiden sich erheblich (Windeler 2008). Nach Schätzungen der EMA sind 5.000-8.000 aller bekannten Krankheiten als 
selten anzusehen. Das Spektrum seltener Krankheiten umfasst Krankheiten mit geringer Inzidenz und kurzem Überleben (z.B. schweres kombiniertes Immundefizienz-Syndrom), Krankheiten mit geringer Inzidenz und langem Überleben (z.B. Duchennne-Muskeldystrophie) und Krankheiten mit relativ hoher Inzidenz und sehr kurzem Überleben (z.B. Pankreaskarzinom, Glioblastom) (Anonym, AMB 2008).

Medikamente gegen seltene Krankheiten wurden in der Vergangenheit gar nicht oder nur sehr selten zur Marktreife entwickelt. Einerseits, weil wegen geringer Patientenzahlen klinische Studien kaum realisiert werden konnten, und andererseits, weil der Markt so klein ist, dass sich die Entwicklungskosten für Arzneimittelhersteller nicht lohnen. Um die notwendige Entwicklung solcher Arzneimittel für die Hersteller interessanter zu machen, kann seit 2000 ein Wirkstoff von der EMA zunächst ,die Ausweisung als Arzneimittel für seltene Leiden“( „Orphan-Drug-Status“) erhalten. Dies garantiert dem PU u.a. ein zehnjähriges Marktexklusivitätsrecht innerhalb der EU ab Marktzulassung, die Befreiung oder Ermäßigung von Gebühren beim Zulassungsverfahren und eine beschleunigte Bearbeitung des Zulassungsantrags. Die Bedingungen hierfür wurden in der EU durch die Verordnung (EG) 141/2000 geregelt.

Mittlerweile hat sich der Markt mit OD sehr stark entwickelt, und es gibt unter ihnen bereits einige Blockbuster. In der EU (Stand: 30. September 2010) waren 62 Arzneimittel als OD zugelassen worden. Fast die Hälfte dieser OD werden zur Behandlung bzw. supportiven Begleittherapie von Tumorerkrankungen eingesetzt. Durch zugelassene oder nicht zugelassene (Off-label) Ausweitung der Anwendungsgebiete ist der Markt mit OD mittlerweile auch für größere PU sehr lukrativ geworden, auch weil es - zumindest in Deutschland bis 2011 - keine Möglichkeit gab, überprüfend und regulierend in die Preisgestaltung für OD einzugreifen.

Auf Probleme bei der Zulassung, Nutzenbewertung und Preisgestaltung von OD und auf Missbrauch infolge der im Rahmen der Verordnung (EG) Nr. 141/2000 geschaffenen Anreize durch PU haben verschiedene Autoren hingewiesen (z.B. Joppi 2008, Windeler 2008, Ferner 2010). Da Patienten mit seltenen Krankheiten denselben Anspruch auf Qualität, Unbedenklichkeit und Wirksamkeit von Arzneimitteln wie andere Patienten haben, wurde beispielsweise kritisiert, dass sie durch Verzicht auf valide Erkenntnisse aus klinischen Studien mit hoher Ergebnissicherheit (z.B. prospektive vergleichende Interventionsstu- 
dien) schlechter gestellt und einem höheren Risiko ausgesetzt werden als Patienten mit nicht selten Krankheiten (Windeler 2008). Darüber hinaus wurde gefordert, die Anreize für die Entwicklung von OD weniger großzügig zu gewähren und sich dabei stärker an dem durch klinische Studien belegten Nutzen zu orientieren (Ferner 2010).

Im ursprünglichen Gesetzesentwurf zum AMNOG war vorgesehen, dass für alle Arzneimittel mit neuen Wirkstoffen bzw. bei neuen Anwendungsgebieten bereits zugelassener Wirkstoffe ab 2011 eine frühe Nutzenbewertung erfolgen soll. In einem Änderungsantrag der Fraktionen der CDU/CSU und FDP wurde dann jedoch im $\S 35 a$ Absatz 1 ein Satz angefügt, der besagt, dass bei OD der medizinische Zusatznutzen durch die Zulassung belegt ist und Nachweise zum medizinischen Nutzen bzw. Zusatznutzen im Dossier des PU nicht vorgelegt werden müssen. Diese Änderung wurde damit begründet, dass bei Arzneimittel für die Behandlung einer seltenen Erkrankung der im Rahmen der arzneimittelrechtlichen Zulassung erbrachte Wirksamkeitsnachweis grundsätzlich als $\mathrm{Zu}$ satznutzen im jeweiligen Anwendungsgebiet anzuerkennen ist und für die Behandlung dieser Erkrankung keine therapeutisch gleichwertige Alternative zur Verfügung steht. Gegen diese Ausnahmeregelung für OD haben sich zahlreiche Institutionen im Gesundheitswesen, darunter auch das Institut für Qualität und Wirtschaftlichkeit im Gesundheitswesen (IQWiG) und die AkdÄ, ausgesprochen, da weder Aspekte des (Zusatz-)Nutzens gemessen an patientenrelevanten Endpunkten und an sinnvollen Vergleichstherapien bei der Zulassung von OD regelhaft geprüft wurden, noch grundsätzlich davon auszugehen ist, dass es für die Behandlung seltener Krankheiten keine therapeutisch gleichwertigen Alternativen gibt (Joppi 2008, Windeler 2010). Im endgültigen Gesetzesentwurf wurde daraufhin im § 35a eine weitere Änderung eingefügt und vom PU eines OD, dessen Umsatz in den letzten 12 Kalendermonaten einen Betrag von 50 Mio. $€$ übersteigt, der Nachweis des medizinischen Zusatznutzens gegenüber der zweckmäßigen Vergleichstherapie gefordert. Für OD mit einem jährlichen Umsatz $\leq 50$ Mio. $€ / J a h r$ müssen entsprechende Nachweise jedoch nicht vorgelegt werden. Dies bedeutet, dass die Durchführung einer Frühbewertung des (Zusatz) Nutzens abhängt vom Umsatz des OD in der GKV, nicht jedoch von der in $\mathrm{Zu}$ lassungsstudien nachgewiesenen Wirksamkeit und Sicherheit bzw. von der Durchführung kontrollierter klinischer Studien im Vergleich zu bereits zugelassenen medikamentösen Therapiealternativen. Dies bedeutet auch, dass für die Verhandlungen über den Erstattungsbetrag bei OD mit einem Umsatz $\leq 50$ Mio. $€ / J a h r$ keine Prüfung durch den G-BA bzw. das IQWiG erfolgt, ob durch diese 
Wirkstoffe eine therapeutische Verbesserung für Patientengruppen oder Anwendungsgebiete gegenüber einer zweckmäßigen Vergleichstherapie erreicht werden kann.

\section{Einschränkung oder Ausschluss der Verordnung von Arzneimitteln bei fehlendem Nachweis des therapeutischen Nutzens oder bei Un- zweckmäßigkeit}

Im $\S 92$ des SGB V wurde bisher festgelegt, dass der GBA über Richtlinien beschließen kann, die eine ausreichende, zweckmäßige und wirtschaftliche Versorgung der Versicherten garantieren. Dabei konnte er auch die Verordnung von Arzneimitteln einschränken oder ausschließen, wenn der therapeutische Nutzen, die medizinische Notwendigkeit oder die Wirtschaftlichkeit nicht nachgewiesen sind. In einem Änderungsantrag der Fraktionen CDU/CSU und der FDP vom 25.10.2010 zu Artikel 1 Nr. 13 ( 92 SGB V) wurde die bisherige Formulierung im $\S 92$ dahingehend verändert, dass der GBA die Verordnung von Arzneimitteln nur einschränken oder ausschließen kann, wenn die Unzweckmäßigkeit erwiesen ist. Gleichzeitig wurde unter Punkt (2 a) ergänzt: „Der Gemeinsame Bundesausschuss kann im Einzelfall mit Wirkung für die Zukunft vom pharmazeutischen Unternehmer im Benehmen mit der Arzneimittelkommission der deutschen Ärzteschaft und dem Bundesinstitut für Arzneimittel und Medizinprodukte oder dem Paul-Ehrlich-Institut innerhalb einer angemessen Frist ergänzende versorgungsrelevante Studien zur Bewertung der Zweckmäßigkeit eines Arzneimittels fordern". Das Nähere zu den Voraussetzungen, zu der Forderung ergänzender Studien, zu Fristen sowie zu den Anforderungen an die Studien wird vom GBA in seiner Verfahrensordnung geregelt. Begründet wurde diese Ergänzung damit, dass die Aussagekraft der für die Zulassung durchgeführten klinischen Studien über die in der Praxis auftretenden Anforderungen an ein Arzneimittel und auch zu patientenrelevanten Endpunkten nach Ansicht der Experten gering ist und deshalb mehr versorgungsrelevante Studien gefordert werden.

Bei vielen neuen Wirkstoffen (z.B. in der Onkologie) wird auf Grundlage der kurz nach Zulassung vom PU vorgelegten Nachweise unter Berücksichtigung der Studienqualität, der Validität der herangezogenen Endpunkte sowie der Evidenzstufe nur schwierig zu bewerten sein, ob und in welchem Ausmaß ein Zusatznutzen vorliegt (s. Verfahrensordnung GBA). Deshalb gewinnt die „Comparative Effectiveness Research“ (CER), für die in den USA mit dem 
„American Recovery and Reinvestment Act“ 2009 1,1 Mrd. US-Dollar bereitgestellt wurden, deutlich an Bedeutung. Das Konzept der CER beruht auf der Tatsache, dass häufig für die medikamentöse Behandlung von Krankheiten mehr als ein Arzneimittel zu Verfügung steht, die für die Arzneimittelverordnung zuständigen Ärzte jedoch nicht wissen, welche Therapie die am besten geeignete ist. Sog. ,head-to-head“ Vergleiche werden vor Zulassung neuer Wirkstoffe häufig nicht durchgeführt (siehe 1.) bzw. vergleichen den neuen Wirkstoff mit einem zugelassenen Arzneimittel, das zum Zeitpunkt der Zulassung des neuen Wirkstoffs nicht mehr dem medizinischen Standard entspricht. Deshalb sind nach $\mathrm{Zu}$ lassung weitere vergleichende klinische Studien erforderlich, die „head-to-head“ den therapeutischen Stellenwert des neuen Wirkstoffs unter Alltagsbedingungen anhand von relevanten Endpunkten einschließlich Lebensqualität und auch unter Berücksichtigung von Aspekten der Kosten-Nutzen-Relation ermitteln. Wenn die og. Formulierung im $\S 92$ des SGB V derartige Untersuchungen mit Instrumenten der CER induziert und die hierfür erforderlichen finanziellen Ressourcen zur Verfügung stehen, würde dies zweifelsfrei einen Fortschritt für die Qualität und Sicherheit der Arzneimitteltherapie in Deutschland bedeuten. Die PU werden hoffentlich vor dem Hintergrund der Verhandlungen über den Erstattungsbetrag bzw. der im $\S 92$ des SGB V vorgesehenen Möglichkeit, weitere versorgungsrelevante Studien bei unklarer Zweckmäßigkeit des neuen Arzneimittels zu fordern, ihre bisher verfolgte Strategie ändern und mehr Geld in Forschung und Entwicklung neuer Arzneimittel investieren, die klinische Forschung auf Bereiche mit tatsächlich vorhandenem Bedarf (,unmet medical need") und hoher Wahrscheinlichkeit für den Nachweis eines Zusatznutzens konzentrieren, sowie die Entwicklung von „me too" Wirkstoffen bzw. Scheininnovationen reduzieren (Chalkidou 2010). Die auch aus Sicht der PU gesehenen Potenziale für CER sollten konsequent genutzt werden, um das derzeit nicht vorhandene Gleichgewicht zwischen Qualität der Arzneimitteltherapie, Kosten für neue Wirkstoffe und Entwicklung echter innovativer Arzneimittel wieder herzustellen (Murray u. McElwee 2010).

Am Beispiel in der Onkologie soll kurz skizziert werden, welche Änderungen in der Entwicklung neuer Wirkstoffe erforderlich sind, um einen therapeutischen Nutzen nachzuweisen. Eine Verbesserung der Datenlage zur Bewertung des Nutzens und der Kosten-Nutzen-Relation neu zugelassener Arzneimittel in der Onkologie ist für eine rationale Verordnung sehr kostenintensiver Wirkstoffe in der Onkologie unverzichtbar. Dafür müssen zunächst Defizite (z.B. vorzeitiger Studienabbruch nach Zwischenanalysen, Zulassung basierend auf post-hoc Sub- 
gruppenanalysen, keine Untersuchung der Lebensqualität) in den klinischen Studien vor der Zulassung abgebaut bzw. die heute gültigen Empfehlungen der EMA konsequenter werden (Schott 2011). Außerdem sollten sich das Studiendesign und die Veröffentlichung von Ergebnissen streng an Leitlinien orientieren, Surrogatmarker als Endpunkte von klinischen Studien nur dann eingesetzt werden, wenn sie mit dem Gesamtüberleben und der Lebensqualität der Patienten korrelieren, und zur Erhebung von Daten zur Lebensqualität oder Symptomkontrolle validierte Instrumente verwendet werden. Unabhängige klinische Studien nach der Zulassung zu noch offenen versorgungsrelevanten Fragen im Rahmen der CER sind unverzichtbare Voraussetzung für einen bedarfsorientierten Einsatz medikamentöser onkologischer Therapiestrategien. Gerade für Studien bei seltenen Krankheiten, wie akute Leukämien, sind alternative Studiendesigns und Endpunkte besonders relevant. Die stärkere Zusammenarbeit der EMA mit PU, kooperativen Studiengruppen sowie „Health Technology Assessment"- (HTA) Organisationen und eine mit der Zulassung verbundene Verpflichtung zu nachfolgenden Versorgungsstudien bieten sich in dieser Situation an (Eichler 2010).

\section{Veröffentlichung der Ergebnisse klinischer Prüfungen}

Das AMNOG beinhaltet auch eine Änderung des AMG. Im $\S 42 \mathrm{~b}$ des AMG wird vom PU verlangt, dass Berichte über alle Ergebnisse konfirmatorischer klinischer Prüfungen zum Nachweis der Wirksamkeit und Unbedenklichkeit der zuständigen Bundesbehörde zur Eingabe in die Datenbank nach § 67a (Datenbankgeschütztes Informationssystem) innerhalb von 6 Monaten nach Erteilung der Zulassung oder der Genehmigung für das in Verkehr bringen zur Verfügung zu stellen sind. Bei klinischen Prüfungen bei einem bereits zugelassenen Arzneimittel hat der Sponsor die Ergebnisse der klinischen Prüfung innerhalb eines Jahres nach ihrer Beendigung zur Verfügung zu stellen. Im Absatz 3 des $\S 42 \mathrm{~b}$ wird ausdrücklich gefordert, dass die Ergebnisse der klinischen Prüfungen unabhängig davon, ob sie günstig oder ungünstig sind, in den Berichten enthalten sein müssen. Ferner werden Aussagen zu nachträglichen wesentlichen Prüfplanänderungen sowie Unterbrechungen und Abbrüchen der klinischen Prüfung in dem Bericht verlangt.

Durch die im AMG jetzt vorgeschriebene Veröffentlichung klinischer Prüfungen wird die Transparenz hinsichtlich der Studienergebnisse verbessert. Diese wichtigen Änderungen im AMG waren notwendig, um unterschiedlichen 
Formen des Bias vorzubeugen (Übersicht bei McGauran 2010). Verschiedene Untersuchungen, so auch eine im Auftrag der Bundesärztekammer von der AkdÄ durchgeführte systematische Literaturübersicht (Schott 2010a,b) konnten zeigen, dass sich die Finanzierung einer Arzneimittelstudie durch ein PU von der Planung über die Durchführung bis hin zur Auswertung und Publikation auf verschiedene Bereiche im Ablauf einer Studie auswirken kann und häufig zu einem für den pharmazeutischen Sponsor positiven Ergebnis führt. Negative und statistisch nicht-signifikante Ergebnisse werden ebenso wie Kenntnisse über UAWs von PU mitunter zurückgehalten. Als Autoren von Publikationen der Studienergebnisse werden anerkannte Meinungsbildner eingeladen, welche die Seriosität der dargestellten Ergebnisse unterstreichen sollen. Dies führt dazu, dass sich neue Arzneimittel verordnende Ärzte nicht immer auf die publizierte Fachliteratur verlassen können. Wichtige Grundlagen der evidenzbasierten Medizin, wie systematische Übersichtsarbeiten und Metaanalysen, aber auch Leitlinien, die auf der Basis dieser publizierten Daten erarbeitet werden und die Therapieentscheidungen bei Patienten bestimmen, können durch die verschiedenen Formen von Bias, einschließlich der häufig bei den beteiligten Autoren bestehenden Interessenkonflikten, beeinflusst werden (Spielmans u. Parry 2010). Dies hat häufig zur Folge, dass Patient nicht angemessen über Nutzen und Risiken von Arzneimitteltherapien informiert werden (Gigerenzer u. Muir Gray 2011).

Um Publikationsbias und die daraus resultierenden Nachteile für NutzenRisiko-Bewertung neuer Arzneimittel, systematische Übersichtsarbeiten bzw. Metaanalysen und evidenzbasierte Therapieempfehlungen in Zukunft besser zu vermeiden, ist zu fordern, dass nicht nur alle Studienergebnisse, sondern auch das Studienprotokoll in einem zentralen, der Öffentlichkeit zugänglichen und vom PU unabhängigen Register eingesehen werden können und an dieser Stelle auch die Registrierung aller klinischen Studien mit Arzneimitteln in Deutschland erfolgt.

\section{Was benötigen wir zusätzlich zum AMNOG für eine optimierte Ein- führung neuer Arzneimittel in die Versorgung?}

Die im AMNOG vorgesehene Frühbewertung des Nutzens und die darauf basierende Vereinbarung eines Erstattungsbetrags zwischen GKV-SV und PU sind ein erster wichtiger Schritt auf dem Weg zu einer optimierten Markteinführung von neuen Arzneimitteln in Deutschland und Preisregulierung bei patentge- 
schützten Arzneimitteln, die keiner Festbetragsgruppe zugeordnet werden können. Dieses Gesetz soll ab Anfang 2011 in Deutschland umsetzen, was in mehreren europäischen Ländern (z.B. Belgien, Frankreich, Österreich, Schottland) bereits seit Jahren, wenn auch unter unterschiedlichen nationalen Rahmenbedingungen, im Rahmen der Entscheidung über die Erstattungsfähigkeit eines neuen Arzneimittels und Festsetzung des Preises praktiziert wird (Sermet 2010; Zentner \& Busse 2011). Dabei wird auch in Deutschland die Frühbewertung des Nutzens zwangsläufig aus einem anderen Blickwinkel (z.B. Bewertung des $\mathrm{Zu}$ satznutzens gegenüber der zweckmäßigen Vergleichstherapie) als dem der nationalen Zulassungsbehörden bzw. der EMA erfolgen. Damit die frühe Nutzenbewertung jedoch nicht nur zur Preisregulierung und Kostenkontrolle genutzt wird, sondern auch einen Beitrag zur Verbesserung der Qualität der Arzneimitteltherapie leisten kann, sind weitere, im AMNOG leider nicht ausreichend berücksichtigte Maßnahmen erforderlich.

Die nach 3 bzw. 6 Monaten im AMNOG vorgesehene Veröffentlichung der Nutzenbewertung erlaubt eine unabhängige Information von Ärzten und Apothekern, aber auch der Öffentlichkeit, über die bei Zulassung verfügbaren wissenschaftlichen Erkenntnisse zu neuen Wirkstoffen bzw. neuen Anwendungsgebieten bereits zugelassener Wirkstoffe. Gleichzeitig kann besser als bisher auf die nur begrenzt zur Verfügung stehenden Daten zur Wirksamkeit und Sicherheit sowie den häufig umstrittenen bzw. noch nicht untersuchten Zusatznutzen neuer Wirkstoffe im Vergleich zu den vorhandenen medikamentösen Therapiealternativen hingewiesen werden. Von den Berichten des G-BA werden sicherlich auch unabhängige, zur „International Society of Drug Bulletins“ gehörende Informationsblätter und die $\mathrm{AkdÄ} \mathrm{profitieren,} \mathrm{deren} \mathrm{wesentliches} \mathrm{Ziel} \mathrm{es} \mathrm{ist,}$ Empfehlungen zum rationalen Einsatz neuer Wirkstoffe auszusprechen und über deren neu bekannt gewordene Risiken rasch nach Zulassung zu informieren. In einem kürzlich im N. Engl. J. Med. erschienenen Perspektivartikel wurde die Frage gestellt: „Teaching Clinicians about Drugs - 50 Years Later, Whose Job Is It?" und gleichzeitig kritisiert, dass nach Zulassung neuer Arzneimittel die von PU zur Verfügung gestellten Informationen häufig unzureichend, mitunter auch irreführend sind, da sie primär Marketingziele verfolgen (Avorn 2010). Es bleibt abzuwarten, ob der leider weiterhin von PU dominierten Fortbildung über neue Wirkstoffe und daraus resultierenden Beeinflussung der Verschreibung neuer Arzneimittel durch Ärzte durch die im Rahmen der frühen Nutzenbewertung gewonnenen unabhängigen Erkenntnisse wirksam begegnet werden kann. 
Ein weiterer positiver Aspekt der Preisvereinbarungen auf Basis der Frühbewertung des Nutzens könnte sein, dass PU ihre derzeitige, eher an kommerziellen als an Patienteninteressen orientierte Vorgehensweise (z.B. neue Wirkstoffe schnell und zu hohen Preisen auf den Markt bringen) im Rahmen der Zulassungsstudien überdenken müssen. Die heute z.B. für Spezialpräparate häufig vom PU verlangten sehr hohen Preise sollten in Zukunft nur akzeptiert werden, wenn der (Zusatz-)Nutzen neuer Wirkstoffe gegenüber bereits verfügbaren Arzneimitteln durch adäquates Design (z.B. Verminderung von Placebokontrollierten Studien, Verzicht auf nicht patientenrelevante Surrogatendpunkte, Nachweis der Überlegenheit anstatt von Äquivalenz bzw. nicht nachgewiesene Unterlegenheit neuer Wirkstoffe) belegt werden konnte. Darüber hinaus werden PU ihre Anstrengungen verstärken müssen, um anhand von validierten Parametern (z. B. Biomarker) Patientengruppen frühzeitiger zu identifizieren, die von neuen Wirkstoffen tatsächlich profitieren. Der geringe Anteil (ca. 30\%) neuer Wirkstoffe mit therapeutisch relevantem Nutzen für Patienten an der Gesamtzahl jährlich neu zugelassener Wirkstoffe würde sicherlich steigen und die Zahl an Scheininnovationen abnehmen.

Die im Rahmen des AMNOG nicht vorgesehene Einführung eines nationalen Horizon Scanning-Systems (HSS) für die frühe Identifizierung und Evaluierung neuer Arzneimittel wäre eine wichtige Ergänzung der jetzt vorwiegend auf dem Dossier des PU basierenden frühen Nutzenbewertung gewesen. HSS sind heute bereits in verschiedenen europäischen Ländern eingeführt und dort vorwiegend bei HTA-Organisationen angesiedelt. Diese Früherkennungssysteme konzentrieren sich auf neue Arzneimittel bzw. neue Anwendungsgebiete zugelassener Arzneimittel, bei denen in naher Zukunft eine Zulassung bzw. -serweiterung zu erwarten ist und die deshalb einer raschen Bewertung ihres therapeutischen ( $\mathrm{Zu}$ satz-)Nutzens und ökonomischer (Budget-) Auswirkungen unterzogen werden sollten. Das Horizon Scanning beginnt zu unterschiedlichen Zeitpunkten, in der Regel 6-36 Monate vor der Zulassung und nutzt unterschiedliche Datenquellen (z.B. vom pharmazeutischen Hersteller, von den Zulassungsbehörden, wissenschaftliche Publikationen, Kongresspräsentationen). Wesentliches Ziel des Horizon Scanning ist die Bereitstellung unabhängiger Informationen bei Markteintritt, die bei der Planung eines optimierten Einsatzes bzw. Entscheidungen über die Preisbildung und Aufnahme neuer Arzneimittel in den Leistungskatalog herangezogen werden können. 
Damit die im AMNOG formulierten Ziele, wie z.B. „den Menschen müssen im Krankheitsfall die besten und wirksamsten Arzneimittel zur Verfügung stehen sowie die Preise und Verordnungen von Arzneimitteln müssen wirtschaftlich und kosteneffizient sein“, tatsächlich erreicht werden, bedarf es neben der konsequenten Umsetzung der auf der Basis der Frühbewertung des Nutzens erfolgenden Vereinbarung von Erstattungsbeträgen für alle neuen Arzneimittel, die keiner Festbetragsgruppe zugeordnet werden können, jedoch weiterer Maßnahmen (Garattini u. Chalmers 2009). Sie betreffen sowohl die konsequente Beachtung der Anforderungen an die Zulassung von Arzneimitteln mit neuen Wirkstoffen als auch die rasche Durchführung unabhängiger klinischer Studien nach der Zulassung. Im Mittelpunkt aktueller Diskussionen, die auch auf der Ebene der EMA geführt werden, stehen die wissenschaftlichen und methodischen Anforderungen an den besseren Nachweis eines patientenrelevanten Nutzens von neuen Wirkstoffen bereits bei der Zulassung im Vergleich zu den verfügbaren medikamentösen bzw. nicht-medikamentösen Therapiealternativen, aber auch Kosten-Nutzen-Bewertungen. Darüber hinaus wird auch nachgedacht über eine stärkere Einbeziehung unabhängiger Patientengruppen in Zulassungsentscheidungen und eine größere Transparenz hinsichtlich der Rohdaten klinischer Zulassungsstudien, Studienprotokolle bzw. Studienergebnisse sowie aller verfügbaren wissenschaftlichen Erkenntnisse, auf denen die Zulassung bzw. Verweigerung der Zulassung basiert.

\section{Literatur}

Anonym (2008): Medikamente für seltene Krankheiten (Orphan drugs). AMB 42: $73-74$

Anonym (2010): Gesetz zur Neuordnung des Arzneimittelmarktes: harmonischer Dreiklang oder eintönige Preisregulierung?. AMB 44: 89-93

Anonym (2011): Where will new drugs come from? Lancet 377: 97

Avorn J (2011): Teaching clinicians about drugs - 50 years later, whose job is it? N. Engl. J. Med. 364: 1185-1187

BMG (2010): http://www.bundesgesundheitsministerium.de/SharedDocs/Presse mitteilungen/DE/2010/pm-10-11-11-AMNOG.html 
Chalkidou, K. (2010): The (possible) impact of comparative effectiveness research on pharmaceutical industry decision making. Clin Pharmacol Ther 87: 264-266

Eichler, H.-G., Bloechl-Daum, B., Abadie, E. et al. (2010): Relative efficacy of drugs: an emerging issue between regulatory agencies and third-party payers. Nature Rev Drug Discov 9: 277-291

Ferner, R.E., Hughes, D.A. (2010): The problem of orphan drugs. Incentives to make orphan drugs should be proportionate to their benefits. BMJ 341: 10591060

Garattini, S., Chalmers, I (2009): Patients and the public deserve big changes in evaluation of drugs. BMJ 338: 804-806

Giezen, T.J., Mantel-Teeuwisse, A.K., Strauss, S.M.J.M. et al. (2008): Safetyrelated regulatory actions for biologicals approved in the United States and the European Union. JAMA 300: 1887-1896

Gigerenzer, G, Muir Gray, JA (2011): Better doctors, better patients, better decisions. The MIT Press, Cambridge

Hess R (2011): Die Frühbewertung des Nutzens neu zugelassener Arzneimittel. GGW 11: 8-14

Honig, P.K. (2011): Comparative Effectiveness: The fourth hurdle in drug development and a role for clinical pharmacology. Clin Pharmacol Ther 89: 183188

Hughes B (2009): 2008 FDA drug approvals. Nature Rev Drug Discov 8: 93-96

Joppi, R., Bertele, V., Garattini, S. (2008): Orphan drug development is not taking off. Br J Clin Pharmacol 67: 494-502

Kaitin KI, DiMasi JA (2011): Pharmaceutical innovation in the 21st century: New drug approvals in the first decade, 2000-2009. Clin Pharmacol Ther 89: 151-155

Light, D.W., Warbuton, R. (2011): Demythologizing the high costs of pharmaceutical research. BioSocieties 6:34-50 
McGauran N, Wieseler B, Kreis J, Schüler V-B, Kölsch H, Kaiser T (2010) Reporting bias in medical research - a narrative review. Trials 11: 37-

Murray, RK, McElwee, NE (2010): Comparative effectiveness research. Critically intertwined with health care reform and the future of biomedical innovation. Arch Intern Med 170:596-599

Okie, S. (2010) Reviving the FDA. N Engl J Med 363: 492-1494

Owens J (2007): 2006 drug approvals: finding the niche. Nature Rev Drug Discov 6: 9-101

Policy Department A (Environment, Public Health and Food Safety (2011): Differences in costs of and access to pharmaceutical products in the EU. http://www.europarl.europa.eu/activities/committees/studies.do?language=EN

Schott, G., Pachl, H., Limbach, U., Gundert-Remy, U., Ludwig, W.-D., Lieb, K. (2010a): The financing of drug trials by pharmaceutical companies and its consequences. Part 1: A qualitative, systematic review of the literature on possible influences on the findings, protocols, and quality of drug trials. Dtsch Arztebl Int 107: 279-285

Schott, G., Pachl, H., Limbach, U., Gundert-Remy, U., Lieb, K, Ludwig, W.-D. (2010b): The financing of drug trials by pharmaceutical companies and its consequences. Part 2: A qualitative, systematic review of the literature on possible influences on authorship, access to trial data, and trial registration and publication. Dtsch Arztebl Int 107: 295-301

Schott, G., Gökbuget, N., Pachl, H., Ludwig W.-D. (2011): Klinische Studien in der Onkologie - Defizite und Lösungsvorschläge. Z Evid Fortbild Qual Gesundh wesen, in 105: Epub 12.02.2011. doi:10.1016/j.zefq.2011.01.006

Schwabe, U., Paffrath, D.: Arzneiverordnungs-Report 2010. Springer-Verlag Heidelberg Berlin, 2010.

Sermet, C., Andrieu, V., Godman, B. et al. (2010):Ongoing pharmaceutical reforms in France. Implications for stakeholders groups. Appl Health Econ Helath Policy 8:7-24 
Spielmans GI, Parry PI (2010) From evidence-based medicine to marketingbased medicine: Evidence from internal industry documents. J Bioeth Inq; 7: 1319

Windeler, J, Lange, S. (2008): Nutzenbewertung in besonderen Situationen Seltene Errkankungen. Z Evid. Fortbild. Qual. Gesundh.wesen 102:25-30

Windeler, J., Koch, K., Lange, S., Ludwig, W.-D. (2010): Zu guter Letzt ist alles selten. Dtsch Arztebl 107: A2032-2034

Zentner A, Busse R (2011): Bewertung von Arzneimitteln - wie gehen andere Länder vor? GGW 11:25-34 


\title{
Erstattungsbedingungen nach dem Arzneimittelmarktneuordnungsgesetz (AMNOG)
}

\author{
Johann-Magnus von Stackelberg und Anja Olbrich
}

\section{Einleitung}

Arzneimittel außerhalb des Festbetragsmarktes tragen wesentlich zum Anstieg der Ausgaben in der GKV bei: sie stellen rund 30 Prozent der Verordnungen, zeigen sich aber für fast 65 Prozent der Arzneimittelausgaben verantwortlich (GKV-Spitzenverband, 2011). Der GKV-Arzneimittelindex zeigt seit Jahren steigende Preise im Nicht-Festbetragsmarkt, wohingegen die Preise im Festbetragsmarkt ständig sinken (Schwabe und Paffrath, 2010). Aufgrund gesetzlicher Vorgaben zur Gruppenbildung sind patentgeschützte Arzneimittel weniger von Festbeträgen erfasst. Die Kosten je Tagesdosis (DDD) bei patentgeschützten Analogpräparaten sind entsprechend doppelt so hoch im Vergleich zum Gesamtmarkt $(1,61 €$ vs. $0,83 €)$. Arzneimittel mit einer innovativen Struktur bzw. einem innovativen Wirkprinzip mit therapeutischer Relevanz sind bezogen auf den Gesamtmarkt fast neun Mal so teuer (7,16 $€$ vs. $0,83 €)$.

Das vom Deutschen Bundestag verabschiedete Arzneimittelmarktneuordnungsgesetz (AMNOG) bringt seit 1. Januar 2011 umfassende strukturelle Änderungen für den Arzneimittelmarkt. Nach der Bewertung des Zusatznutzens für neu zugelassene Arzneimittel mit neuen Wirkstoffen durch den Gemeinsamen Bundesausschuss führt der GKV-Spitzenverband mit den pharmazeutischen Unternehmern Verhandlungen zu GKV-weit gültigen Erstattungsbeträgen. Kommt eine Vereinbarung nicht zu Stande, setzt eine Schiedsstelle den Erstattungsbetrag unter Berücksichtigung der tatsächlichen Abgabepreise in anderen europäischen Ländern fest. Abweichend von bestehenden Vereinbarungen oder Schiedssprüchen können einzelne Krankenkassen oder ihre Verbände Vereinbarungen über die Erstattung von Arzneimitteln und zur Versorgung ihrer Versicherten mit Arzneimitteln treffen.

Diese Neuregelungen sind darauf ausgerichtet, das Preismonopol der pharmazeutischen Unternehmer zu brechen. Die freie Preisbildung besteht damit nur noch für Arzneimittel mit geringfügigen Ausgaben für die GKV und für alle anderen nicht festbetragsfähigen Arzneimittel im ersten Jahr nach Inverkehrbringen. Auf der anderen Seite schränkt das AMNOG die Möglichkeiten des Ge- 
meinsamen Bundesausschusses bei Verordnungseinschränkungen und ausschlüssen ein. Der nunmehr erforderliche Beweis der Unzweckmäßigkeit wird in vielen Fällen nur über (noch durchzuführende) Versorgungsstudien zu erbringen sein. Dies schenkt der Pharmaindustrie viel Zeit zur Vermarktung fraglicher Produkte. Eine „4. Hürde“, als konsequenten Weg zur Umsetzung einer wirtschaftlichen Arzneimittelversorgung, scheint derzeit nicht durchsetzbar und politisch nicht gewollt.

\section{Frühe Zusatznutzenbewertung und Erstattungsbeträge}

Spätestens zum Zeitpunkt ihres Inverkehrbringens ist für alle neu zugelassenen Arzneimittel mit neuen Wirkstoffen eine Bewertung des Zusatznutzens auf der Basis eines Herstellerdossiers durchzuführen. Das dreimonatige Bewertungsverfahren liegt in der Verantwortung des Gemeinsamen Bundesausschusses. Nach insgesamt sechs Monaten beschließt der Gemeinsame Bundesausschuss insbesondere über den Zusatznutzen und die Anforderungen für eine qualitätsgesicherte Anwendung des Arzneimittels (siehe Abbildung 1).

Abbildung 1: Das Bewertungs- und Erstattungsverfahren nach dem AMNOG

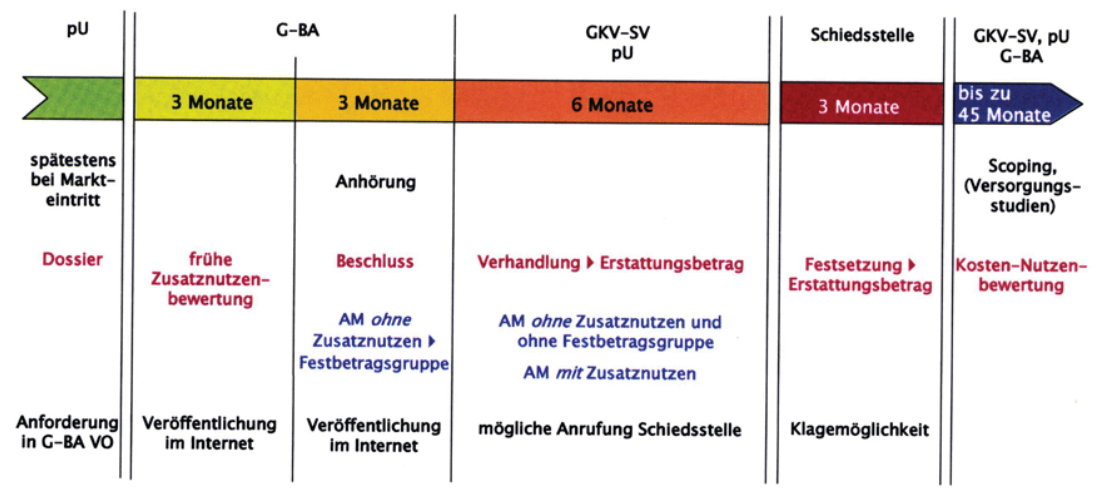

Die Bewertung des Zusatznutzens nach § 35a SGB V folgt der ArzneimittelNutzenbewertungsverordnung (AM-NutzenV). Für Arzneimittel, die pharmakologisch-therapeutisch vergleichbar mit Festbetragsarzneimitteln sind, ist der medizinische Zusatznutzen als therapeutische Verbesserung nach nachzuweisen. Der Nachweis erfolgt aufgrund der Fachinformationen und durch Bewertung von klinischen Studien nach methodischen Grundsätzen der evidenzbasierten Medizin, soweit diese Studien allgemein verfügbar sind oder gemacht werden 
und ihre Methodik internationalen Standards entspricht. Für alle anderen Arzneimittel wird ein Zusatznutzen für das jeweilige Anwendungsgebiet nachgewiesen im Vergleich zu der vom Gemeinsamen Bundesausschuss bestimmten zweckmäßigen Vergleichstherapie. Basis sind hier die arzneimittelrechtliche Zulassung, die behördlich genehmigten Produktinformationen sowie Bekanntmachungen von Zulassungsbehörden und wiederum die Bewertung von klinischen Studien nach den internationalen Standards der evidenzbasierten Medizin. Der Zusatznutzen eines Arzneimittels gegenüber einer zweckmäßigen Vergleichstherapie wird gemessen in patientenrelevanten therapeutischen Effekten insbesondere hinsichtlich der Verbesserung des Gesundheitszustands, der Verkürzung der Krankheitsdauer, der Verlängerung des Überlebens, der Verringerung von Nebenwirkungen oder der Verbesserung der Lebensqualität.

Arzneimittel ohne feststellbaren Zusatznutzen werden unmittelbar der Festbetragsregelung zugeführt, sofern sie nach den gesetzlichen Einordnungskriterien dazu geeignet sind. Der Festbetrag wird auf Basis der geltenden Regressionsgleichung ermittelt und kann dann spätestens neun Monate nach Inverkehrbringen der Arzneimittel in Kraft treten.

Für Arzneimittel, die nicht den Festbeträgen zuweisbar sind, hat der GKVSpitzenverband nach $\S 130 \mathrm{~b}$ SGB V mit dem pharmazeutischen Unternehmer innerhalb von sechs Monaten Erstattungsbeträge in Gestalt von Rabatten zu vereinbaren (siehe Abbildung 1)

Dabei erhalten Arzneimittel ohne Zusatznutzen und ohne Festbetragsgruppe einen Erstattungsbetrag, der zu nicht höheren Kosten führt als die der zweckmäBige Vergleichstherapie. Nach der AM-NutzenV werden die Therapiekosten durch den Apothekenabgabepreis und ggf. zusätzliche Kosten weiterer Leistungen bei regelhaft notwendiger Inanspruchnahme nach der Fachinformation bestimmt. Sobald eine Festbetragsgruppenbildung möglich ist, kann der GKVSpitzenverband die Vereinbarung außerordentlich kündigen, sodass der Festbetrag den Erstattungsbetrag ersetzt.

Für Arzneimittel mit Zusatznutzen führt der GKV-Spitzenverband mit dem jeweiligen pharmazeutischen Unternehmer Verhandlungen über die Höhe des Rabatts auf den Abgabepreis und die näheren Anforderungen an die Verordnung. Im Erstattungsbetrag werden sowohl die Jahrestherapiekosten der zweckmäßigen Vergleichstherapie als auch die Wahrscheinlichkeit, das Ausmaß und 
die therapeutische Bedeutung des Zusatznutzens nach dem Beschluss des Gemeinsamen Bundesausschusses Berücksichtigung finden. Im Fall eines Zusatznutzens in nur einem von mehreren zugelassenen Anwendungsgebieten gibt es für den Erstattungsbetrag prinzipiell zwei Optionen: Er wird berechnet 1. als gewichteter Zuschlag auf die Kosten der Therapiealternative oder 2. als höherer Zuschlag für die betreffende Patientengruppe unter Verordnungsausschluss für alle anderen Patientengruppen. Die Verordnungsanforderungen, welche Vorgaben zu Zweckmäßigkeit, Qualität und Wirtschaftlichkeit einer Verordnung beinhalten, werden den Therapiehinweisen des Gemeinsamen Bundesausschusses folgen

Bei neuen Arzneimitteln, die nicht einer Vergleichstherapie bewertend gegenübergestellt werden können, fehlt die Bezugsgröße für den Erstattungsbetrag. In diesen Fällen sind für den Erstattungsbetrag die tatsächlichen Abgabepreise in anderen europäischen Ländern bedeutsam.

Bei den Vereinbarungen ist es aus Sicht der GKV auch erforderlich, den Erstattungsbetrag an die erwartete Verordnungsmenge zu knüpfen. Der Beschluss des G-BA zur Anzahl der Patienten und Patientengruppen mit bedeutsamem Zusatznutzen kann dabei dem Mengengerüst zugrunde gelegt werden. Zur konkreten Umsetzung sind außer Staffelrabatten auch Mengenvereinbarungen vorstellbar, die bei Überschreitung zu Rückzahlungen oder zur Anrechnung auf einen neu zu vereinbarenden Rabatt führen. Eine weitere Möglichkeit bietet die Kündigungsoption nach einem Jahr.

Kommt es in den Verhandlungen über den Erstattungsbetrag nicht zu einer Einigung zwischen GKV-Spitzenverband und pharmazeutischen Unternehmern, erfolgt die Festsetzung durch eine neu einzurichtende Schiedsstelle innerhalb von drei Monaten. Der festgesetzte Erstattungsbetrag gilt ab dem 13. Monat nach erstmaligem Inverkehrbringen. Die vom GKV-Spitzenverband und den maßgeblichen Organisationen der pharmazeutischen Unternehmer auf Bundesebene gebildete Schiedsstelle soll die Höhe der tatsächlichen Abgabepreise in anderen Ländern berücksichtigen.

Auch wenn in den Verhandlungen die tatsächlich entstehenden Kosten für die GKV zu berücksichtigen sind, liegt zum Zeitpunkt der ersten Verhandlung keine Kosten-Nutzen-Bewertung vor. Nach dem AMNOG ist eine Kosten-NutzenBewertung auch nur auf Verlangen und Kosten eines pharmazeutischen Unter- 
nehmers im Falle keines festgestellten Zusatznutzens oder auf Antrag der Parteien nach Festsetzung des Erstattungsbetrags durch die Schiedsstelle vorgesehen. Mit verwertbaren Ergebnissen ist damit frühestens nach weiteren 9 und spätestens nach weiteren 45 Monaten zu rechnen (siehe Abbildung 1).

Abbildung 2 ordnet den neuen Erstattungsregelungen nach dem AMNOG die charakteristische Umsatzentwicklung eines neuen Arzneimittels zu. Nach Wille et al. (2003) sind die entscheidenden Umsatzphasen mit positivem Umsatzwachstum, innerhalb der ersten sechs Jahre nach Inverkehrbringen zu erwarten. Entsprechend kann sich ein neues Arzneimittel bei frühester Festlegung eines Erstattungsbetrages nach einem Jahr bereits im Markt etabliert haben. Eine Änderung des Verordnungsverhaltens der Ärzte sowie damit verbunden eine Umstellung der Patienten dürfte in einer solchen Situation nicht unproblematisch sein. Verlangt ein pharmazeutischer Unternehmer eine Kosten-NutzenBewertung unmittelbar nach Abschluss der Nutzenbewertung, fiele das Ergebnis gerade zusammen mit der Festlegung des ersten Erstattungsbetrages. Dieser könnte entsprechend außerordentlich gekündigt werden und eine zweite Verhandlungsrunde mit möglichem Schiedsverfahren würde beginnen. Ein Bewertungs- und Verhandlungsmarathon ist daher absehbar. Aus einer Kosten-NutzenBewertung unter Berücksichtigung von Versorgungsstudien kann ein Erstattungsbetrag im Extremfall erst nach sechs Jahren folgen. Zu diesem Zeitpunkt sind die entscheidenden Umsatzphasen bereits gelaufen.

Nach § 130c SGB V können einzelne Krankenkassen oder ihre Verbände abweichend von bestehenden Vereinbarungen oder Schiedssprüchen mit pharmazeutischen Unternehmern Vereinbarungen über die Erstattung von Arzneimitteln und zur Versorgung ihrer Versicherten mit Arzneimitteln treffen. Dabei kann insbesondere eine mengenbezogene Staffelung des Preisnachlasses, ein jährliches Umsatzvolumen mit Ausgleich von Mehrerlösen oder eine Erstattung in Abhängigkeit von messbaren Therapieerfolgen vereinbart werden. Durch eine solche Vereinbarung kann die Vereinbarung des GKV-Spitzenverbands ergänzt oder abgelöst werden. 
Abbildung 2: Das AMNOG und die Entwicklungsphasen eines patentgeschützten Arzneimittels

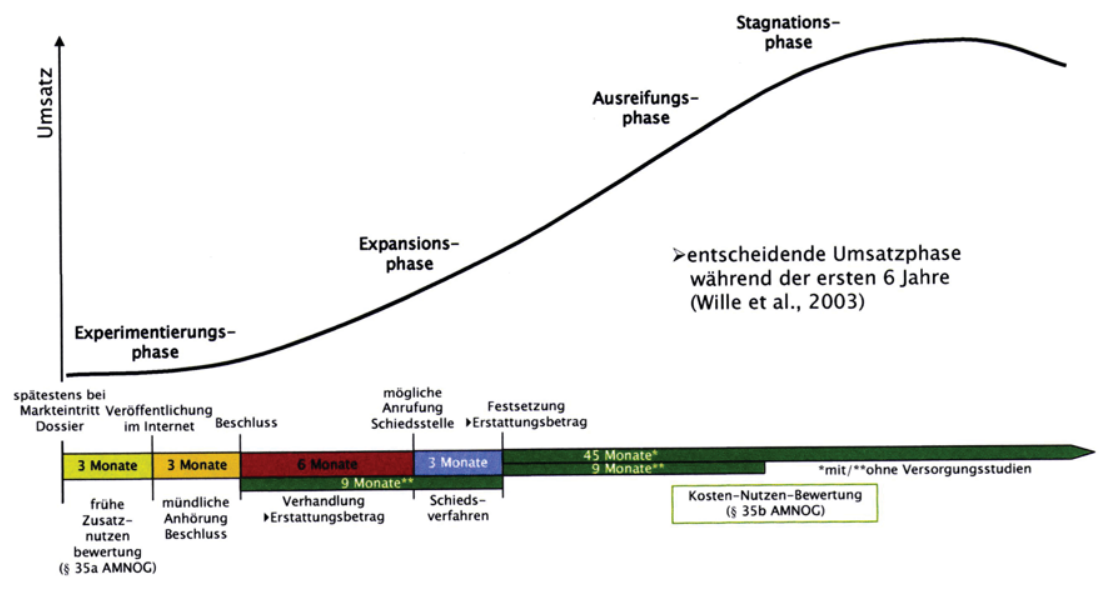

\section{Fazit und Ausblick}

Die Zusatznutzenbewertung von Arzneimitteln mit neuen Wirkstoffen bei Markteintritt ist grundsätzlich zu begrüßen. Sie ermöglicht zu einem deutlich früheren Zeitpunkt als bisher eine Aussage über den Stellenwert eines Arzneimittels im Markt. Leider bleibt das vorhandene Zeitfenster zwischen Zulassung und Markteintritt für eine Bewertung ungenutzt. Dies bedeutet, dass Ärzte und Patienten nach wie vor im ersten Jahr nach Inverkehrbringen des Arzneimittels einer vermeidbaren Unsicherheit in der Therapie ausgesetzt sind. Auch wäre auf Basis der frühen Nutzenbewertung umgehend eine Kosten-Nutzen-Bewertung anzuschließen. Es ist bedauerlich, dass im Rahmen der Frühbewertung Verordnungsausschlüsse nicht zulässig sind. Dies wäre in jedem Fall angebracht, wenn der Nutzen des bewerteten Arzneimittels geringer als der Nutzen der zweckmäBigen Vergleichstherapie ist.

Die Verhandlung zum Erstattungsbetrag erfolgt auf Grundlage des Beschlusses des Gemeinsamen Bundesausschusses über die Nutzenbewertung. Darüber hinaus können die tatsächlichen Abgabepreise in anderen europäischen Ländern berücksichtigt werden. Sowohl eine unzureichende Evidenz zum Zusatznutzen als auch ein geringer Referenzpreis im Ausland hätten damit zwangsläufig einen niedrigen Erstattungsbetrag zur Folge. Verbessert sich die Evidenz im Laufe der 
Zeit, ist eine Erhöhung des Erstattungsbetrags möglich. In diesem Sinn folgt im Arzneimittelmarkt zukünftig das Geld der Leistung, sodass Anreize zur Verbesserung der Studienlage und zur Entwicklung von Arzneimitteln mit hohem $\mathrm{Zu}$ satznutzen gesetzt werden.

Die Erstattungsbeträge nach $\S 130 \mathrm{~b}$ SGB V wie auch die Abschläge für verschreibungspflichtige Arzneimittel nach $\S 130$ a SGB V werden nach dem AMNOG nicht nur den gesetzlichen Krankenkassen sondern auch den Unternehmen der privaten Krankenversicherung zu Gute kommen. Da die PKV nicht dem Solidaritätsgrundsatz verpflichtet ist und, ganz im Gegenteil, Rosinenpickerei bei der Versichertenauswahl betreibt, bedeutet dies einen klaren Wettbewerbsnachteil für die GKV. Wie auch die Aufhebung der Sperrfrist von drei Jahren zum Verlassen der GKV, hilft dies die Beitragsfehlkalkulation und eigentliche Unwirtschaftlichkeit der PKV zu kaschieren.

Das AMNOG verhält sich ambivalent zu den Arzneimittelrabattverträgen der einzelnen Krankenkassen. Einerseits klärt es mit $\S 130$ c SGB V eindeutig das Verhältnis zwischen Selektivverträgen und Vereinbarungen des GKVSpitzenverbands und sichert damit ein weiteres Vertragsfeld. Andererseits schwächt es mit Anwendung des Kartellverbots in $\S 69$ SGB V und der Mehrkostenregelung auf diese Selektivverträge nach $\S 13$ SGB V die Möglichkeiten der Krankenkassen, durch eigene Rabattverhandlungen zusätzliche Einsparvolumina zu generieren

\section{Literatur}

Arzneimittel-Nutzenbewertungsverordnung - AM-NutzenV. Bundesgesetzblatt vom 28.12.2010

Arzneimittelmarktneuordnungsgesetz - AMNOG. BT Drucksache 17/3698. Bundesgesetzblatt vom 22.12.2010.

Schwabe U., Paffrath D., Hrsg., 2010. Arzneiverordnungs-Report 2010. Heidelberg: Springer Medizin Verlag.

Wille E., Erbsland M., Ulrich V., 2003. Zur Berechnung einer Innovationskomponente auf dem Arzneimittelmarkt 1995-2002. Gutachten im Auftrag von GlaxoSmithKline GmbH \& Co. KG. 
Eberhard Wille and Klaus Knabner - 978-3-631-75550-1 Downloaded from PubFactory at 01/11/2019 03:39:43AM via free access 


\section{Effekte der Frühbewertung patentgeschützter Arzneimittel auf die for- schende pharmazeutische Industrie}

\section{Franz Waibel}

In Deutschland wird derzeit eine frühe Zusatznutzenbewertung patentgeschützter Arzneimittel eingeführt. Die Entwicklung der Gesundheitsausgaben der vergangenen Jahre erfordert aus Sicht der Fraktionen von CDU/ CSU und FDP weitere Maßnahmen zur Begrenzung der Gesundheitsausgaben. ${ }^{1}$

Diese Ziele und die damit verbundenen Maßnahmen werden im Grundsatz von Bayer akzeptiert, und wir sind bereit, einen Beitrag zur Reform des Arzneimittelmarktes zu leisten. Allerdings hat die Frühbewertung erhebliche Konsequenzen für forschende Arzneimittelhersteller und damit auch Bayer ebenso wie für den Innovationsstandort Deutschland. Nicht alle diese Konsequenzen sind unvermeidlich. Auch eine schnelle Frühbewertung sollte als Maßnahme einer Kosten-Nutzen-Bewertung unterzogen werden: Neben den erwarteten positiven Folgen für die Arzneimittelausgabenentwicklung müssen die negativen Folgen für die Innovationsaktivitäten in Deutschland berücksichtigt werden. Bayer verfügt über umfangreiche Erfahrungen mit internationalen Arzneimittelbewertungen, und wir sind bereit, uns konstruktiv einzubringen und mitzuhelfen, die schnelle Frühbewertung sinnvoll auszugestalten.

Dabei sollte sich die Qualität des Prozesses der schnellen Frühbewertung an folgenden Maßstäben messen lassen:

- Gleichbehandlung aller Gesundheitstechnologien

- Transparenz im Bewertungsprozess

- faire Berücksichtigung von Unsicherheitsfaktoren zum Zeitpunkt der Markteinführung

Oberstes Bewertungskriterium innerhalb des Prozesses sollte der Nutzen einer Innovation für Patienten und die Gesellschaft sein. Eine Betrachtung allein aus Sicht der gesetzlichen Krankenversicherung ist zu eng.

\footnotetext{
${ }^{1}$ Siehe dazu den Entwurf eines Gesetzes zur Neuordnung des Arzneimittelmarktes in der gesetzlichen Krankenversicherung (Arzneimittelmarktneuordnungsgesetz - AMNOG) vom 6.7.2010 (BT-Drucksache 17/2413).
} 
Vor diesem Hintergrund wird nachfolgend zunächst der vom Gesetzgeber vorgesehene Prozess der schnellen Frühbewertung vorgestellt. Anschließend werden die Konsequenzen dieses Prozesses für forschende Arzneimittelhersteller und den Innovationsstandort Deutschland diskutiert. Darauf basierend werden Vorschläge zur sinnvollen Ausgestaltung des Prozesses aus der Sicht von Bayer abgeleitet.

\section{Der Prozess der schnellen Frühbewertung knüpft an die Zulassung eines Arzneimittels an}

Im Anschluss an die Zulassung eines neuen Arzneimittels findet künftig in Deutschland eine Frühbewertung statt. Diese Bewertung folgt der vorherigen intensiven Bewertung durch die internationalen Arzneimittelzulassungsbehörden. Im Rahmen der Zulassung von Arzneimitteln werden Wirksamkeit, Unbedenklichkeit und die pharmazeutische Qualität des jeweiligen Arzneimittels geprüft und abgewogen. Es werden folglich nur solche Arzneimittel in den Markt eingeführt, deren positive Wirkungsbilanz bereits gezeigt wurde. Diese Zulassung erfordert bestimmte Studiendesigns, wie zum Beispiel plazebokontrollierte Studien, damit eine sichere Einschätzung des Wirkungsprofils erfolgen kann. Diese erste Form der Nutzenbewertung ist für die pharmazeutischen Hersteller ein zwingender Schritt.

Zum Zeitpunkt der Arzneimittelzulassung in Deutschland ist der größte Teil der vorliegenden Daten also bereits bewertet worden. Die Frühbewertung ergänzt eine zweite Interpretation aus einer anderen Perspektive, aber man darf nicht vergessen, auf Basis der im Wesentlichen gleichen Daten. Umfangreiche Zusatzinformationen, wie zum Beispiel vergleichende Studien zwischen neuen Behandlungsformen, liegen in der Regel nicht vor. Dies ist dadurch begründet, dass nur bereits zugelassene Behandlungen / Arzneimittel als Vergleichsgruppe in Zulassungsstudien in Frage kommen. Ergänzend zu den Zulassungsinformationen enthält daher das Dossier, das der Hersteller des neu zugelassenen Arzneimittels beim G-BA einreicht, Informationen über zugelassene Anwendungsgebiete, erwarteten medizinischer Nutzen, erwarteten medizinischen Zusatznutzen im Verhältnis zur zweckmäßigen Vergleichstherapie, Anzahl der Patienten und Patientengruppen, für die ein therapeutisch bedeutsamer Zusatznutzen erwartet wird, sowie die geschätzten Therapiekosten für die gesetzliche Krankenversicherung. 
Die Frühbewertung soll nach dem Willen des Gesetzgebers dafür sorgen, dass im Krankheitsfall den Patienten die besten und wirksamsten Arzneimittel zur Verfügung stehen. Außerdem sollen die Preise und Verordnungen von Arzneimitteln wirtschaftlich und kosteneffizient sein. Daneben sollen verlässliche Rahmenbedingungen für Innovationen, die Versorgung der Versicherten und die Sicherung von Arbeitsplätzen geschaffen werden. Eine genauere Analyse dieser Ziele, die in sich nicht widerspruchsfrei sein können, soll an dieser Stelle nicht erfolgen. Aber wer Kosteneffizienz fordert, wird akzeptieren müssen, dass es dafür nicht unbedingt die besten Arzneimittel gibt. Dies wird davon abhängen, wie Kosteneffizienz beurteilt werden soll und welche Kriterien angelegt werden.

Wirft man einen Blick auf die Erfahrungen in anderen Ländern, dann kann dieser Prozess nur dann reibungslos funktionieren, wenn bereits vor dem Beginn der pivotalen Zulassungsstudien verbindlich zwischen dem Gemeinsamen Bundesausschuss und den jeweiligen Unternehmen vereinbart ist, was zur Frühbewertung herangezogen werden wird. Insbesondere die Bestimmung der zweckmäßigen Vergleichstherapie muss frühzeitig erfolgen, so dass eine Berücksichtigung dieser Vergleichstherapie im Rahmen klinischer Studien möglich wird. Eine Ausweitung der frühen Zusammenarbeit zwischen Zulassungsbehörden und Unternehmen auf die Arzeimittelbewertungsbehörden ist in diesem Zusammenhang ein zentraler Schritt. Da hohe Investitionen damit verbunden sind, müssen aus den gemeinsamen Planungen bindende Absprachen hervorgehen.

Auf der Basis der Frühbewertung erfolgt dann entweder die Einordnung der neuen Substanz in das deutsche Festbetragssystem oder es folgen Preisverhandlungen zwischen dem Hersteller und dem GKV-Spitzenverband bei festgestelltem Zusatznutzen. Am Ende des Verhandlungsprozesses steht eine Vereinbarung, die sowohl für die GKV als auch für die privaten Krankenversicherungen gilt (§ 78 (3a) AMG). Der Prozess der schnellen Frühbewertung regelt also nicht nur die öffentlich-rechtliche Seite der Kostenübernahme für das neue Arzneimittel, sondern greift auch in die Rechtsverhältnisse zwischen Herstellern und privaten Krankenversicherungen ein. Das ist ordnungspolitisch nicht nachvollziehbar: Warum unterstützt der Gesetzgeber private Krankenversicherungsunternehmen bei ihrem Bemühen um Gewinnerzielung?

Im Falle der Gewährung von Rabatten bei Abgabe des Arzneimittels durch den Hersteller führt die Geltungserstreckung auf die PKV dazu, dass die Rabatte schnell öffentlich bekannt werden, so dass im internationalen Zusammenhang 
die rabattierten Preise, also die Netto-Preise, bekannt werden. Diese Vorgehensweise hat Auswirkungen auf die Preisbildung in vielen vor allem europäischen Märkten aber oftmals auch auf Länder in anderen Kontinenten, die Deutschland als Referenzland heranziehen. Dies ist ein erheblicher Eingriff in die Preishoheit der Hersteller, nicht nur auf der nationalen Ebene, und beeinflusst die wirtschaftlichen Erfolge und die Innovationskraft der pharmazeutischen Industrie. Schon in der Vergangenheit haben sich die damit befassten öffentlichen Stellen in anderen europäischen Ländern für die gewährten gesetzlich vorgeschriebenen und die freiwillig vereinbarten Rabatte interessiert, um so die tatsächlichen Herstellerabgabepreise ermitteln zu können. Da die freiwillig vereinbarten Rabatte nicht öffentlich zugänglich waren, konnten folglich nur die gesetzlich vorgeschriebenen Rabatte berücksichtigt werden. Durch die neue Transparenz wird die Bereitschaft der Hersteller sinken, der GKV zusätzliche Rabatte einzuräumen, da diese zu einer Absenkung des europäischen Preisniveaus führen.

\section{Neue Arzneimittel müssen sich heute schon in vielen Ländern einer Bewer- tung stellen}

Der Bewertungsvorgang an sich ist für forschende Arzneimittelhersteller nichts Neues. Solche Prozesse werden bereits heute in zahlreichen anderen Ländern unter Beteiligung der Hersteller durchgeführt. Allerdings sind die Prozesse von Land zu Land sehr unterschiedlich ausgestaltet. Das führt zu erheblichem Aufwand bei den Herstellern, die französische, englische, schottische, schwedische, deutsche und viele andere Anforderungen zeitgleich berücksichtigen müssen. Dies lässt sich anschaulich darstellen anhand der Vergleichssubstanz, die bei Wirksamkeitsvergleichen herangezogen werden kann. Je nach Land gibt es da erhebliche Unterschiede, wie die nachfolgende Übersicht zeigt. ${ }^{2}$

\footnotetext{
${ }^{2}$ Die Übersicht ist übersetzt aus Sculpher MJ, Drummond MF. Analysis sans frontiers: can we ever make economic evaluations generalisable across jurisdictions? Pharmacoeconomics. 2006;24(11):1087-99, p. 1095. Die Autoren selbst zitieren mit der Übersicht die Ergebnisse einer Umfrage der International Society for Pharmacoeconomics and Outcomes Research im Bereich ihrer Mitglieder.
} 
Tabelle 1: Übersicht zu den unterschiedlichen Auswahlmethoden von zweckmäßigen Vergleichstherapien

\begin{tabular}{|l|l|}
\hline Empfohlene Vergleichssubstanz ( $\mathbf{n}=\mathbf{2 7}$ ) & Zahl der Guidelines \\
\hline Am häufigsten eingesetztes Medikament & 8 \\
\hline Effektives Medikament oder Minimalbehandlung & 2 \\
\hline $\begin{array}{l}\text { Jedes Medikament ist möglich, aber die Wahl muss begründet wer- } \\
\text { den }\end{array}$ & 1 \\
\hline Standardbehandlung und keine Behandlung & 2 \\
\hline $\begin{array}{l}\text { Häufigstes Medikament, günstiges Medikament und keine Behand- } \\
\text { lung }\end{array}$ & 1 \\
\hline $\begin{array}{l}\text { Häufigstes / günstigstes / effektives Medikament und keine Be- } \\
\text { handlung }\end{array}$ & 2 \\
\hline Häufigstes / günstigstes / effektives Medikament & 1 \\
\hline Behandlung, die voraussichtlich ersetzt wird & 1 \\
\hline Effizienteste / Effektivste Behandlung und keine Behandlung & 2 \\
\hline Alle relevanten Vergleiche & 2 \\
\hline Effektivste Behandlung und keine Behandlung & 1 \\
\hline Keine klare Vorgabe & 3 \\
\hline
\end{tabular}

Aus der Tabelle 1 wird deutlich, dass die Vorgaben zur zweckmäßigen Vergleichstherapie in den untersuchten Guidelines zur Bewertung Arzneimitteln erheblich variieren. Besonders häufig ist das am häufigsten eingesetzte Medikament die zweckmäßige Vergleichstherapie, mitunter aber auch das wirksamste Arzneimittel und das Placebo. Die Bandbreite in der Übersicht zeigt, dass nur in Ausnahmefällen die zweckmäßige Vergleichstherapie in verschiedenen Ländern die gleiche sein wird. Ein gemeinsamer Nenner ist eher nicht zu erwarten. Dies führt für die Hersteller zu nationalen Besonderheiten in den Bewertungen, die den Aufwand zur Einführung eines neuen Arzneimittels weiter erheblich steigern.

\section{Forschende Arzneimittelhersteller wie Bayer akzeptieren die Nutzenbewer- tung}

Die vielfältigen Unterschiede zwischen den Ländern sind für forschende Arzneimittelhersteller aufwendig. Die Hersteller führen bereits heute im Rahmen der Entwicklung neuer Arzneimittel eine Reihe von Studien durch, die allein dem Zweck dieser landesspezifischen Nutzenbewertungen dienen: 
So werden beispielsweise Patientenpräferenzen als so genannte UtilityStudies erhoben. Im Rahmen solcher Studien wird etwa die Frage gestellt, was dem Patienten wichtiger ist: die Verlängerung des Lebens oder die Lebensqualität. Eine andere Fragestellung versucht, die Patientenpräferenzen gegenüber bestimmten Nebenwirkungen bei geringerem Schlaganfallsrisiko zu ermitteln. Zudem werden Studien zu Erfassung des direkten patientenrelevanten Nutzens durchgeführt.

Da nicht für alle möglichen Vergleichssubstanzen klinische Studien durchgeführt werden können, werden indirekte Vergleichsstudien initiiert. Dies ist erforderlich, weil die Kosten direkter Vergleichsstudien hoch sind und mitunter viel Zeit vergehen muss, bis die Studienergebnisse vorliegen. Außerdem befinden sich häufig die Vergleichssubstanzen von Wettbewerbern noch in der Phase der klinischen Forschung und stehen damit nicht zur Verfügung, etwa weil die Zulassung fehlt.

Darüber hinaus werden Studien zu den Krankheitskosten in den jeweiligen Indikationen durchgeführt. Diese Studien erfordern eine aufwändige und detaillierte Beobachtung des aktuellen Behandlungsgeschehens und eine detaillierte Kostenerfassung. Krankheitskostenstudien werden mit der Einführung der Frühbewertung durch den Gesetzgeber gefordert, wenn nach den Kosten der Therapie einer bestimmten Erkrankung für die GKV gefragt wird. ${ }^{3}$ Die für die Beantwortung dieser Frage erforderlichen Daten liegen in der Regel nirgendwo vor. Die Hersteller müssen folglich Krankheitskosten in hinreichend große Stichproben von Patienten beobachtet und dokumentieren.

Daneben sind in epidemiologischen Studien Prävalenz und Inzidenz einer Erkrankung, zunehmend sogar in einer genau bestimmten Patientengruppe mit dieser Erkrankung, zu erheben. Diese Studien sind Grundlage von Modellen zur Einschätzung der Ausgabenwirkungen des neuen Arzneimittels (Budget impactModelle). Das Gesetz fordert die Abschätzung der Ausgabenwirkungen eines neuen Arzneimittels im einzureichenden Dossier im Rahmen der Frühbewertung.

\footnotetext{
${ }^{3}$ Bei der Antwort auf die Frage nach den Therapiekosten wird man zumindest die vermiedenen Kosten durch die neue Therapie an anderer Stelle in der Gesundheitsversorgung mit berücksichtigen müssen. Ein „Brutto-Kosten“-Vergleich stünde dem Ziel des Gesetzentwurfs, eine kosteneffiziente Versorgung mit Arzneimitteln zu erreichen, entgegen.
} 
Die Ausgabenwirkungen neuer Therapien und der jeweiligen zweckmäßigen Vergleichstherapie können nur errechnet werden, wenn die Abläufe im Behandlungsalltag hinreichend beschrieben sind. Diese Ablaufbeschreibung umfasst Standardtherapien, den schrittweisen Wechsel zu Kombinationstherapien, umfassendere Interventionen, etc. Viele dieser Studien geben umfassende Einblicke in die gesundheitliche Versorgung des jeweiligen Landes und dienen damit weit über die Informationen zum Produkt dem gesundheitspolitischen Allgemeinwohl. Die Kosten solcher Studien sollten entsprechend nicht ausschließlich den pharmazeutischen Herstellern aufgebürdet, sondern gemeinschaftlich von den Partnern in den jeweiligen nationalen Gesundheitswesen geschultert werden.

Forschende Hersteller führen bereits heute Studien im Versorgungsalltag nicht nur nach Zulassung eines neuen Arzneimittels, sondern auch vor dem Abschluss der Entwicklung durch, um Nutzenbewertungen zu ermöglichen. Jede weitere Erhöhung der Datenanforderungen an die Hersteller reduziert die Investitionsmittel, die für Innovationen zur Verfügung stehen. Hier sollte ein Umdenken stattfinden: Wie viel Zusatzinformationen sind zwingend erforderlich, um eine adäquate Entscheidung zu treffen? Diese Frage ist selbst im Rahmen einer Kosten-Nutzen-Abwägung der Arzneimittelbewertung zu prüfen und zu beantworten.

\section{Die Frühbewertung hat erhebliche Konsequenzen für die Unternehmen}

Im Rahmen der Bewertungsprozesse in den verschiedenen Ländern ist immer wieder festzustellen, dass sich Nutzenbegriffe systemabhängig unterscheiden und mal enger und mal weiter gefasst sind. Ein gutes Beispiel ist in diesem $\mathrm{Zu}$ sammenhang der Nutzenbegriff im Zusammenhang mit der Behandlung von Thrombosen. Thrombosen können lebensbedrohende Erkrankungen sein, die zu tödlichen Lungenembolien, zum Schlaganfall oder zu Durchblutungsstörungen der Herzkranzgefäße führen können.

Die aktuellen Standardtherapien weisen Nachteile auf: Bei kurzer Therapiedauer, beispielsweise nach Operationen, wird der Wirkstoff gespritzt. Bei Erkrankungen, die eine langjährige Therapie erfordern, gibt es zwar bereits seit vielen Jahren Medikamente in Tablettenform, Dosierung und Blutspiegel müssen allerdings vom Arzt regelmäßig überwacht werden, damit Thromboseprophylaxe und Blutungsrisiko nicht außerhalb der individuell erforderlichen Balance liegen. 
Bayer führt derzeit ein Studienprogramm mit mehr als 65.000 Patienten durch, in dem der Wirkstoff Rivaroxaban in der Prävention und Therapie eines breiten Spektrums akuter und chronischer thromboembolischer Erkrankungen, beispielsweise zur Schlaganfallprävention bei Patienten mit Vorhofflimmern, geprüft wird. Mit Rivaroxaban werden die Patienten nur einmal täglich ein $\mathrm{Me}-$ dikament nehmen müssen, ohne dass man vielfältige Wechselwirkungen mit anderen Medikamenten und mit Lebensmitteln befürchten muss, die die Wirksamkeit des Thrombosemittels beeinträchtigen können. Folglich würden die regelmäßigen, teilweise mehrmals pro Woche erforderlichen Überprüfungen des Blutgerinnungsstatus durch den Arzt wegfallen.

Welcher Nutzen entsteht nun in den verschiedenen Systemen? Nach welchen Kriterien bewertet die Gesellschaft den Wegfall der regelmäßigen ärztlichen Überwachung und damit eine höhere Akzeptanz der Therapie? Welchen Wert hat für den Patienten die Einnahme einer Tablette im Vergleich zum Spritzen?

Diese Aspekte werden in den unterschiedlichen Gesundheitssystemen ganz, nur teilweise oder gar nicht beachtet. Während in den Systemen, die Lebensqualitätsveränderungen über qualitätsadjustierte Lebensjahre erfassen können, die oben genannten Aspekte zumindest eine gewisse Rolle spielen können, scheinen sie in der nun vorgeschlagenen Frühbewertungsmethodik eher von untergeordneter Bedeutung zu sein. Zumindest legt das die vorgeschlagene Rechtsverordnung nahe. Aus der Sicht von Bayer sollte der Nutzenbegriff weit gefasst werden, dass auch Verbesserungen, die den Patienten direkt zu Gute kommen wie reduzierte Laborkontrollen, weniger Arztbesuche, vereinfachte Arzneimittelanwendung berücksichtigt werden. Auch Kosten die jenseits der GKV entstehen, wie die Kosten für die Patienten, Kosten infolge von reduzierter Arbeitsfähigkeit, Pflege, Reha, Frühverrentung sowie Verminderungen der Belastung von Angehörigen sollten wie in anderen Ländern berücksichtigt werden.

Darüber hinaus ist es ein Unterschied festzustellen, ob ein Zusatznutzen vorliegt, oder zu bewerten, was (uns als Gesellschaft) dieser Zusatznutzen wert ist. Während das Verfahren zur Feststellung eines Zusatznutzens inzwischen stark ausgebaut ist und auch Erfahrungswerte zur Durchführung der Nutzenbewertung vorliegen, ist die Bewertung des Zusatznutzens nicht vorangekommen. Die Diskussion um das Effizienzkurvenkonzept hat gezeigt, dass es bei der Bewertung des Zusatznutzens sehr unterschiedliche Wertansätze geben kann, dass letztlich 
aber eine normative Entscheidung zu fällen ist, bei der bezweifelt werden kann, ob der Gemeinsame Bewertungsausschuss dazu hinreichend legitimiert ist.

Neben den bereits angesprochenen Fragen nach dem Nutzenbegriff und der zweckmäßigen Vergleichstherapie unterscheiden sich auch die Behandlungspfade in den Ländern und damit die Krankheitskosten wie die Tabelle 2 zeigt. So zeigen etwa die Daten des MS-Barometers, ${ }^{4}$ dass in weniger als 10 Ländern mehr als 50\% der MS-Erkrankten krankheitsmodifizierende Medikamente erhalten. Während in Deutschland $70 \%$ der Erkrankten entsprechend therapiert werden, sind es in Polen lediglich 4\%.

Tabelle 2: Übersicht zu den lokalen Unterschieden der erforderlichen Informationen für die Nutzenbewertung

\section{Informationsbedarf Lokale Unterschiede}

Qualitätsadjustierte Einsatz in Großbritannien und Schweden, aber nicht in Lebensjahre (QALY) Frankreich oder Deutschland

Zweckmäßige Ver- Unterschiedliche Ansätze in den Ländern

gleichstherapie - Häufigste Therapie

- Therapie nach Leitlinie

- Billigste Therapie

Behandlungspfade Unterschiedlich in den Ländern

Krankheitskosten Systemabhängig verschieden

Patientenpräferenzen Unterschiedlich in den Ländern

Nutzen unter Alltags- Versorgungsstudien sind systemabhängig bedingungen

Ferner unterscheiden sich die Menschen in dem, was ihnen wichtig ist. ${ }^{5}$ Daher werden im Versorgungsalltag immer wieder andere Versorgungsentscheidungen

4 Vergleiche zum MS-Barometer http://www.dmsg.de/multiple-sklerose-news/index.php ?w3pid=news\&kategorie=ausdembundesverband\&anr=2156 (Zugriff am 6.2.2011).

${ }^{5}$ Ein gutes Beispiel dafür mögen die Erkenntnisse zu den Präferenzen von SchizophreniePatienten sein, die sich erheblich unterschieden. So gab es Patienten, die eher das „NichtAuffallen" im Alltag der Abwesenheit von Symptomen vorgezogen haben. Siehe dazu Rosenheck R et al., (2005), Measuring outcome priorities and preferences in people with schizophrenia, in: The British Journal of Psychiatry, Vol 187, S. 529-536. 
getroffen als etwa Behandlungsleitlinien nahelegen. ${ }^{6}$ Der Nutzen im Versorgungsalltag hängt ebenfalls stark von den jeweiligen Gesundheitssystemen ab: So wird der Nutzen einer Intervention, die Krankenhaustage einspart, in einem System mit geringer stationärer Beanspruchung weniger wert sein, als in einem System mit starker stationärer Beanspruchung. ${ }^{7}$

Es sind also die gleichen Forschungsfragen, die in den verschiedenen Ländern jeweils gesondert untersucht werden müssen, um die Besonderheiten von Gesundheitssystemen, Behandlungskulturen und Patientenpräferenzen berücksichtigen und den jeweiligen Anforderungen an Nutzenbewertungen in den Ländern gerecht werden zu können.

$\mathrm{Zu}$ den steigenden Ausgaben aufgrund erhöhter Informationsbedürfnisse in den Ländern kommt hinzu, dass im Rahmen der Frühbewertung eines neuen Produkts auch bereits bewertete Produkte wieder auf den Prüfstand kommen können, indem sie indirekt als zweckmäßige Vergleichstherapie herangezogen werden können. Darüber hinaus kann der Gemeinsame Bundesausschuss jederzeit eine Nutzenbewertung veranlassen. Beides erfordert eine laufende Aktualisierung des Dossiers bzw. der darin enthaltenen Informationen. Um das zu gewährleisten, müssen nach Markteinführung in der jeweiligen Indikation und zum Produkt umfangreiche weitere Studien und Datenerhebungen durchgeführt werden. Diese erheblichen Investitionen ziehen Ressourcen von der Erforschung und Entwicklung neuer Medikamente ab, und reduzieren damit die Innovationskraft der pharmazeutischen Industrie. Wer wiederholte Bewertungen verlangt, muss die Konsequenzen berücksichtigen.

Noch komplexer wird die Situation, wenn die Anforderungen der Beteiligten im Prozess zum Marktzugang sehr unterschiedlich sind oder sich in manchen Fällen sogar widersprechen. Das Beispiel der pulmonalen Hypertonie zeigt: Zulassungsbehörden möchten den 6 Minutes Walking Distance Test als Endpunkt. Andere Institutionen, die die Nutzenbewertung oder das Health Technology Assessment durchführen, haben diesen Test mehrfach abgelehnt aufgrund von

\footnotetext{
${ }^{6}$ Was sich umgekehrt niederschlägt in der Tatsache, dass Patientenpräferenzen in klinischen Leitlinien wohl nur unzureichend berücksichtigt werden. Siehe dazu Chong $\mathrm{Ch}$ et al., (2009), How Well Do Guidelines Incorporate Evidence on Patient Preferences? In: J Gen Intern Med, Vol 24(8), S. 977-82.

${ }^{7}$ Beispielhaft für eine solche Situation sei hier die Einführung arzneimittelfreisetzender Stents genannt, die in Großbritannien auch Fragen zur Kapazität von Katheterplätzen hervorgerufen hat.
} 
Zweifeln an seiner prognostischen Relevanz. Überlegenheit beim primären Endpunkt in den klinischen Studien ist deshalb nicht immer auch als Überlegenheit in Nutzenbewertungen anerkannt worden (z.B. Ventavis mit Wirkstoff Iloprost zur inhalativen Therapie in der primären pulmonalen Hypertonie (NYHA III)). In solchen Fällen ist eine größere Flexibilität in der Umsetzung von Nutzenbewertungen erforderlich, umso mehr, wenn es sich dabei um seltene Erkrankungen handelt.

Aus Sicht von Bayer sollte ergänzender Informationsbedarf auf den vorhandenen Informationen aufbauen und diese verwerten. Eine Nutzenbewertung zusätzlich zur Zulassung führt in jedem Fall zu höheren Kosten für die Hersteller. Dies gilt auch für die Frühbewertung. Allerdings entstehen zusätzliche Anforderungen und damit höhere Kosten nicht aus dem Bewertungsanspruch an sich, sondern aus den nationalen (deutschen) Besonderheiten und den Unterschieden zwischen Zulassung und Nutzenbewertung.

Die Aussage des Gesetzgebers, dass das bei Markteintritt einzureichende Dossier nicht mehr als 1.250 EUR kostet, basiert zweifellos auf falschen Annahmen. Wie dargelegt, erfordert die Erhebung der spezifischen Daten für Deutschland umfassende Investitionen. Die Zusammenstellung der Daten im Einreichungsdossier ist dann tatsächlich nicht mehr besonders teuer.

Forschung ist ein aufwendiger und unsicherer Prozess. Die Unternehmen verwenden jedes Jahr erhebliche Ressourcen auf die Erforschung und Entwicklung neuer Arzneimittel. Für Bayer belief sich der Aufwand für Forschung und Entwicklung 2009 allein in Deutschland auf fast 1 Milliarde Euro.

Aus der Vergangenheit wissen wir, dass nur eine aus 10.000 untersuchten Substanzen zugelassen wird. Der Entwicklungsprozess eines Arzneimittels dauert im Durchschnitt 12 Jahre und kostet bis zu 1 Mrd. US\$. In der gleichen Zeitspanne (1998 - 2010) hat sich in Deutschland das gesundheitspolitische Umfeld durch mindestens 12 Reformgesetze verändert wie der Tabelle 3 zu entnehmen ist. $\mathrm{Zu}$ den Unsicherheiten im Entwicklungsprozess kommen also erhebliche Unsicherheiten hinsichtlich des künftigen Vermarktungsumfelds für neue Arzneimittel hinzu. Mehr Planbarkeit und verbindliche Absprachen über eine Nutzenbewertung von Arzneimitteln, so wie sie die Verordnung über die Nutzenbewertung von Arzneimitteln nach $\S 35 \mathrm{a}$ Absatz 1 SGB V für Erstattungsvereinbarungen nach $\S 130 \mathrm{~b}$ SGB V (Arzneimittel-Nutzenbewertungsverordnung - 
AM-NutzenV) vorsieht, sind ein Schritt in die richtige Richtung. Wir sehen den frühen Dialog mit dem G-BA als eine Chance, die Unwägbarkeiten für neue Produkteinführungen zu reduzieren. Wenn Studien mit dem Gemeinsamen Bundesausschuss klar vereinbart werden, werden ihre Ergebnisse nach Vorlage vor der gleichen Institution auch akzeptiert, so dass sich weitere Kosten und Zeitverzögerungen vermeiden lassen. Dies ist im Interesse der Hersteller und der Patienten, denen mit schnellen Markteinführungen innovativer Produkte bessere Behandlungsergebnisse beschert werden.

Tabelle 3: Übersicht zu den Reformgesetzen von 1993 bis 2010

\begin{tabular}{|l|l|}
\hline Jahr & Reformgesetz \\
\hline 01.01 .1993 & Gesundheits-Strukturgesetz \\
\hline 01.01 .1997 & Beitragsentlastungsgesetz \\
\hline 01.07 .1997 & GKV-Neuordnungsgesetz \\
\hline 01.01 .1999 & GKV-Solidaritätsstärkungsgesetz \\
\hline 01.01 .2000 & GKV-Gesundheitsreform 2000 \\
\hline 03.08 .2001 & Festbetrags-Anpassungsgesetz \\
\hline 31.12 .2001 & Arzneimittelbudget-Ablösungsgesetz \\
\hline 23.02 .2002 & Arzneimittelausgaben-Begrenzungsgesetz \\
\hline 01.01 .2003 & Beitragssatzsicherungsgesetz \\
\hline 01.01 .2004 & GKV-Modernisierungsgesetz \\
\hline 2006 & Arzneimittelversorgungs-Wirtschaftlichkeitsgesetz \\
\hline 2007 & Vertragsarztrechts-Ånderungsgesetz \\
\hline 2007 & $\begin{array}{l}\text { Gesetz zur Stärkung des Wettbewerbs in der Gesetzlichen Krankenversiche- } \\
\text { rung }\end{array}$ \\
\hline 2010 & GKV-Änderungsgesetz \\
\hline 2010 & Arzneimittelmarkt-Neuordnungsgesetz \\
\hline
\end{tabular}

\section{Die Frühbewertung hat erhebliche Konsequenzen für den Innovations- standort Deutschland}

Im internationalen Vergleich zeigt sich, dass Arzneimittelinnovationen in Deutschland nur sehr zurückhaltend eingesetzt werden. Ärzte sind infolge ihrer Budgetverantwortung oftmals sehr zögerlich in der Verschreibung innovativer Produkten, so dass Patienten auch die Produkte, die einen Mehrnutzen bewiesen haben, in Deutschland seltener verordnet bekommen, als im benachbarten Ausland. Es ist im Interesse von Gesundheitspolitik und Bevölkerung, die Qualität der Versorgung zu verbessern, medizinische Fortschritte zum Wohle der Patienten schneller und umfassender zu nutzen. 
Durch eine Entschlackung der vielfältigen Kostendämpfungsmaßnahmen im Arzneimittelsektor kann die Verunsicherung der verordnenden Ärzte reduziert werden.

Abbildung 1: Übersicht zu den Steuerungsinstrumenten in der GKVArzneimittelversorgung

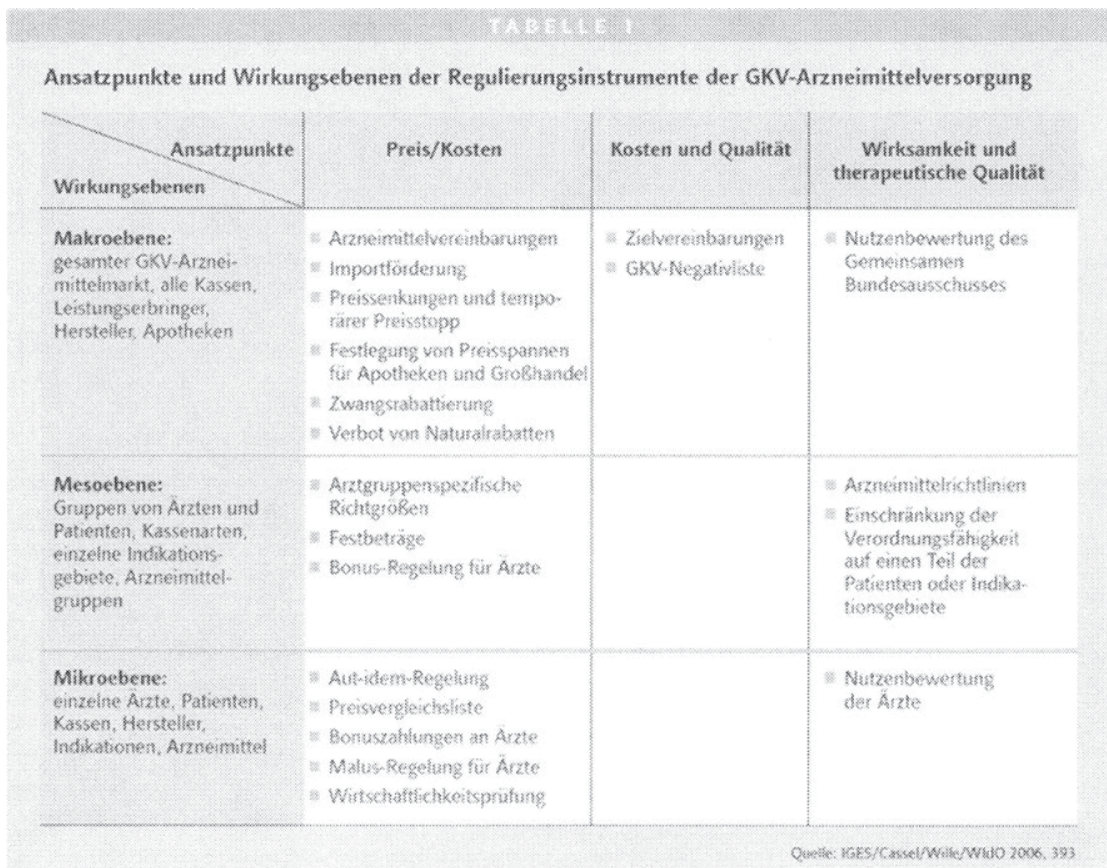

Das Wissen über die Wechselwirkungen dieser vielen Steuerungsinstrumente wie in Abbildung 1 dargestellt ist gering. Ein entsprechendes Gutachten aus dem Jahr 2006 systematisiert diese Regulierungsinstrumente und kommt zu dem Schluss, dass die bestehenden Regulierungen deutlich zurückgeführt werden sollten zugunsten eines Vertragswettbewerbs zur Steuerung der Arzneimittelversorgung in der Gesetzlichen Krankenversicherung. ${ }^{8}$

${ }^{8}$ Siehe dazu Cassel D et al, (2006), Weiterentwicklung wettbewerblicher Strukturen im GKVLeistungsmarkt. Ein interdisziplinäres Gutachten im Auftrag des AOK-Bundesverbandes, Endbericht vom 12. Mai 2006, Bonn. 
Der Gesetzgeber hat mit der Einführung der Frühbewertung die Chance, überholte Steuerungsinstrumente im Arzneimittelmarkt abzuschaffen. Denn ohne Deregulierung wird jede Neuregelung nur zu einem Mehr an Gesetzen. Und damit wird das deutsche Gesundheitssystem nur noch komplizierter. Steigende Komplexität im Regelungssystem führt in der Regel zu steigender Unsicherheit und steigenden Kosten für die davon betroffenen Akteure. Die dafür eingesetzten Mittel stehen dann für Forschung und Entwicklung nicht mehr zur Verfügung.

Regularien mit einem ausschließlichen Budgetfokus können durch die Frühbewertung ersetzt werden, die Mehrnutzen und Mehrkosten in Einklang bringt. Wo ein solches Gleichgewicht entstanden ist, sollten Ärzte nicht weiter durch starre Budgets zur Verschreibung veralteter Produkte bewegt werden.

Forschende Arzneimittelhersteller wie Bayer wollen mithelfen, den geplanten Bewertungsprozess sinnvoll auszugestalten

Vor diesem Hintergrund wollen wir von Bayer, dazu beitragen, den geplanten Bewertungsprozess sinnvoll auszugestalten. Sinnvoll heißt, dass Mehrkosten nur dort verursacht werden, wo dies unumgänglich ist, und Unsicherheiten über das Verfahren soweit als möglich beseitigt werden. Zudem sollten die Risiken für den Standort Deutschland nicht weiter erhöht werden. Daher sollten in der Ausgestaltung der Frühbewertung folgende Punkte berücksichtigt werden:

Die Zulassung muss als erste Form der Arzneimittelbewertung anerkannt werden. Zum Zeitpunkt der Zulassung kann noch nicht klar sein, wie leistungsfähig ein Arzneimittel im Versorgungsalltag ist - dem muss Rechnung getragen werden, in dem Zulassungsstudien sowie weitere verfügbare Daten und entsprechende Modelle herangezogen werden, um Aussagen zum Nutzen eines neuen Arzneimittels ableiten zu können.

Dabei muss der Nutzenbegriff eindeutig und umfassend definiert werden. Die Perspektive bei der Bewertung von Arzneimitteln sollte möglichst weit sein, das heißt, die Sicht von Ärzten, Patienten, gegebenenfalls auch Angehörigen berücksichtigen. Alle relevanten Kosten und Kostenersparnisse müssen berücksichtigt werden, unabhängig davon, wem sie entstehen. Dazu gehört z.B. ein reduzierter Betreuungsbedarf durch den Hausarzt ebenso wie ein verkürzter Krankenhausaufenthalt oder eine schnellere Rückkehr an den Arbeitsplatz. Arznei- 
mittel können helfen, die Gesundheitsausgaben zu vermindern. Ein restriktiver Umgang mit Arzneimitteln kann umgekehrt zum Anstieg von Gesundheitsausgaben führen. ${ }^{9}$

Der Bewertungsprozess muss eindeutig und transparent definiert werden. Rechtzeitig vor dem Beginn eines Bewertungsverfahrens sollte immer ein strukturierter Dialog-Prozess unter Einbeziehung von Patienten, Fachkreisen und Arzneimittelherstellern stehen (Scoping). Eine angemessene Verfahrenstransparenz und Beteiligungsrechte der betroffenen Hersteller müssen gewährleistet sein. Vereinbarungen mit dem Gemeinsamen Bundesausschuss müssen Hersteller zur Verringerung von Unsicherheit einfordern können.

Das Gesetz zur Neuordnung des Arzneimittelmarktes sieht einen Paradigmenwechsel bei Arzneimittelinnovationen vor. Mit der Frühbewertung und der zentralen Verhandlung von Erstattungskonditionen werden Regulierungsinstrumente aus anderen Ländern importiert und zentral etabliert. Zugleich bleibt der umfassende Abbau anderer, preis- und mengenregulierender Instrumente - die in den betreffenden Ländern nicht bestehen - aus. Diese überflüssigen Steuerungsinstrumente müssen abgeschafft werden, zentralistische Elemente im System durch wettbewerbliche ersetzt werden.

Mit diesen Vorschlägen soll erreicht werden, dass mit der schnellen Frühbewertung positive Effekte für die Patientenversorgung erzielt werden können, ohne dass die Nebenwirkungen für die Hersteller den Innovationsstandort Deutschland noch schlechter werden lassen. Für dieses Ziel setzt sich Bayer ein.

${ }^{9}$ Dies belegen Untersuchungen zum Zusammenhang zwischen der Innovationsaktivität im Bereich der Arzneimittel und der Ausgabenentwicklung im jeweiligen Gesundheitssystem. Siehe dazu auch verschiedene Publikation von F. Lichtenberg, insbesondere F. Lichtenberg (2001), Are the benefits of newer drugs worth their cost? Evidence from the 1996 MEPS, in: Health Affairs 20(5), p- 241-51. 


\section{Literaturverzeichnis}

Cassel D et al, (2006), Weiterentwicklung wettbewerblicher Strukturen im GKV-Leistungsmarkt. Ein interdisziplinäres Gutachten im Auftrag des AOKBundesverbandes, Endbericht vom 12. Mai 2006, Bonn.

Chong Ch et al., (2009), How Well Do Guidelines Incorporate Evidence on Patient Preferences? In: J Gen Intern Med, Vol 24(8), S. 977-82.

Lichtenberg, F., (2001), Are the benefits of newer drugs worth their cost? Evidence from the 1996 MEPS, in: Health Affairs 20(5), S. 241-51.

o. V., (2010), Entwurf eines Gesetzes zur Neuordnung des Arzneimittelmarktes in der gesetzlichen Krankenversicherung (Arzneimittelmarktneuordnungsgesetz - AMNOG) vom 6.7.2010 (BT-Drucksache 17/2413).

Rosenheck R et al., (2005), Measuring outcome priorities and preferences in people with schizophrenia, in: The British Journal of Psychiatry, Vol 187, S. 529-536.

Sculpher MJ, Drummond MF, (2006), Analysis sans frontiers: can we ever make economic evaluations generalisable across jurisdictions? In: Pharmacoeconomics; Vol 24(11), S. 1087-99.

Internet:

http://www.dmsg.de/multiple-sklerose-news/index.php?w3pid=news\&kategorie $=$ ausdembundesverband\&anr=2156 (Zugriff am 6.2.2011). 


\section{Verzeichnis der Autoren}

Joachim Bovelet

Vivantes Netzwerk für Gesundheit

Klinikum am Urban

Dieffenbachstraße 1

10967 Berlin

Dr. Maximilian Gaßner

Präsident des Bundesversicherungsamtes

Friedrich-Ebert-Allee 38

53113 Bonn

Dr. Dirk Göpffarth

Bundesversicherungsamt

Friedrich-Ebert-Allee 38

53113 Bonn

Dr. Rolf Hoberg

Vorsitzender des Vorstandes

der AOK Baden-Württemberg

Heilbronner Straße 184

70191 Stuttgart

Professor Dr. Norbert Klusen

Vorsitzender des Vorstandes

der Techniker Krankenkasse

Bramfelder Straße 140

22305 Hamburg

Dr. Klaus Knabner

Kaiserstuhlstraße 3

14129 Berlin

Professor Dr. Wolf-Dieter Ludwig

HELIOS Klinikum Berlin-Buch

Schwanebecker Chaussee 50

13125 Berlin 
Dr. Carl-Heinz Müller

Mitglied des Vorstandes

Kassenärztliche Bundesvereinigung

Herbert-Lewin-Platz 2

10623 Berlin

Professor Dr. Günter Neubauer

Institut für Gesundheitsökonomik

Nixenweg $2 b$

81739 München

Dr. Anja Olbrich

Spitzenverband der Krankenkassen

Mittelstraße 51

10117 Berlin

Dr. Hagen Pfundner

Mitglied des Vorstandes

Roche Pharma AG

Emil-Barell-Straße 1

79639 Grenzach-Wyhlen

Johann-Magnus von Stackelberg

Mitglied des Vorstandes

Spitzenverband der Krankenkassen

Mittelstraße 51

10117 Berlin

Dr. Frank Waibel

Head Global Market Access GMACS

Bayer Schering Pharma AG

Müllerstraße 178

13342 Berlin

Christian Weber

Abteilungsleiter

Bundesministerium für Gesundheit

Friedrichstraße 108

10117 Berlin 
Rüdiger Wittmann

Bundesversicherungsamt

Friedrich-Ebert-Allee 38

53113 Bonn

Manfred Zach

Ministerialdirigent

Ministerium für Arbeit und Sozialordnung, Familien

und Senioren Baden-Württemberg

Schellingstraße 15

70174 Stuttgart 


\section{STAATLICHE ALLOKATIONSPOLITIK IM MARKTWIRTSCHAFTLICHEN SYSTEM}

Band 1 Horst Siebert (Hrsg.): Umweltallokation im Raum. 1982.

Band 2 Horst Siebert (Hrsg.): Global Environmental Resources. The Ozone Problem. 1982.

Band 3 Hans-Joachim Schulz: Steuerwirkungen in einem dynamischen Unternehmensmodell. Ein Beitrag zur Dynamisierung der Steuerüberwälzungsanalyse. 1981.

Band 4 Eberhard Wille (Hrsg.): Beiträge zur gesamtwirtschaftlichen Allokation. Allokationsprobleme im intermediären Bereich zwischen öffentlichem und privatem Wirtschaftssektor. 1983.

Band 5 Heinz König (Hrsg.): Ausbildung und Arbeitsmarkt. 1983.

Band 6 Horst Siebert (Hrsg.): Reaktionen auf Energiepreissteigerungen. 1982.

Band 7 Eberhard Wille (Hrsg.): Konzeptionelle Probleme öffentlicher Planung. 1983.

Band 8 Ingeborg Kiesewetter-Wrana: Exporterlösinstabilität. Kritische Analyse eines entwicklungspolitischen Problems. 1982.

Band 9 Ferdinand Dudenhöfer: Mehrheitswahl-Entscheidungen über Umweltnutzungen. Eine Untersuchung von Gleichgewichtszuständen in einem mikroökonomischen Markt- und Abstimmungsmodell. 1983.

Band 10 Horst Siebert (Hrsg.): Intertemporale Allokation. 1984.

Band 11 Helmut Meder: Die intertemporale Allokation erschöpfbarer Naturressourcen bei fehlenden Zukunftsmärkten und institutionalisierten Marktsubstituten. 1984.

Band 12 Ulrich Ring: Öffentliche Planungsziele und staatliche Budgets. Zur Erfüllung öffentlicher Aufgaben durch nicht-staatliche Entscheidungseinheiten. 1985.

Band 13 Ehrentraud Graw: Informationseffizienz von Terminkontraktmärkten für Währungen. Eine empirische Untersuchung. 1984.

Band 14 Rüdiger Pethig (Ed.): Public Goods and Public Allocation Policy. 1985.

Band 15 Eberhard Wille (Hrsg.): Óffentliche Planung auf Landesebene. Eine Analyse von Planungskonzepten in Deutschland, Österreich und der Schweiz. 1986.

Band 16 Helga Gebauer: Regionale Umweltnutzungen in der Zeit. Eine intertemporale Zwei-Regionen-Analyse. 1985.

Band 17 Christine Pfitzer: Integrierte Entwicklungsplanung als Allokationsinstrument auf Landesebene. Eine Analyse der öffentlichen Planung der Länder Hessen, Bayern und Niedersachsen. 1985.

Band 18 Heinz König (Hrsg.): Kontrolltheoretische Ansätze in makroökonometrischen Modellen. 1985.

Band 19 Theo Kempf: Theorie und Empirie betrieblicher Ausbildungsplatzangebote. 1985.

Band 20 Eberhard Wille (Hrsg.): Konkrete Probleme öffentlicher Planung. Grundlegende Aspekte der Zielbildung, Effizienz und Kontrolle. 1986.

Band 21 Eberhard Wille (Hrsg.): Informations- und Planungsprobleme in öffentlichen Aufgabenbereichen. Aspekte der Zielbildung und Outputmessung unter besonderer Berücksichtigung des Gesundheitswesens. 1986.

Band 22 Bernd Gutting: Der Einfluß der Besteuerung auf die Entwicklung der Wohnungs- und Baulandmärkte. Eine intertemporale Analyse der bundesdeutschen Steuergesetze. 1986.

Band 23 Heiner Kuhl: Umweltressourcen als Gegenstand internationaler Verhandlungen. Eine theoretische Transaktionskostenanalyse. 1987. 
Band 24 Hubert Hombach: Besteuerung, Inflation und Kapitalallokation. Intersektorale und internationale Aspekte. 1987.

Band 25 Peter Müller: Intertemporale Wirkungen der Staatsverschuldung. 1987.

Band 26 Stefan Kronenberger: Die Investitionen im Rahmen der Staatsausgaben. 1988.

Band 27 Armin-Detlef Rieß: Optimale Auslandsverschuldung bei potentiellen Schuldendienstproblemen. 1988.

Band 28 Volker Ulrich: Preis- und Mengeneffekte im Gesundheitswesen. Eine Ausgabenanalyse von GKV-Behandlungsarten. 1988.

Band 29 Hans-Michael Geiger: Informational Efficiency in Speculative Markets. A Theoretical Investigation. Edited by Ehrentraud Graw. 1989.

Band 30 Karl Sputek: Zielgerichtete Ressourcenallokation. Ein Modellentwurf zur Effektivitätsanalyse praktischer Budgetplanung am Beispiel von Berlin (West). 1989.

\section{ALLOKATION IM MARKTWIRTSCHAFTLICHEN SYSTEM}

Band 31 Wolfgang Krader: Neuere Entwicklungen linearer latenter Kovarianzstrukturmodelle mit quantitativen und qualitativen Indikatorvariablen. Theorie und Anwendung auf ein mikroempirisches Modell des Preis-, Produktions- und Lageranpassungsverhaltens von deutschen und französischen Unternehmen des verarbeitenden Gewerbes. 1991.

Band 32 Manfred Erbsland: Die öffentlichen Personalausgaben. Eine empirische Analyse für die Bundesrepublik Deutschland. 1991.

Band 33 Walter Ried: Information und Nutzen der medizinischen Diagnostik. 1992.

Band 34 Anselm U. Römer: Was ist den Bürgern die Verminderung eines Risikos wert? Eine Anwendung des kontingenten Bewertungsansatzes auf das Giftmüllrisiko. 1993.

Band 35 Eberhard Wille, Angelika Mehnert, Jan Philipp Rohweder: Zum gesellschaftlichen Nutzen pharmazeutischer Innovationen. 1994.

Band 36 Peter Schmidt: Die Wahl des Rentenalters. Theoretische und empirische Analyse des Rentenzugangsverhaltens in West- und Ostdeutschland. 1995.

Band 37 Michael Ohmer: Die Grundlagen der Einkommensteuer. Gerechtigkeit und Effizienz. 1997.

Band 38 Evamaria Wagner: Risikomanagement rohstoffexportierender Entwicklungsländer. 1997.

Band 39 Matthias Meier: Das Sparverhalten der privaten Haushalte und der demographische Wandel: Makroökonomische Auswirkungen. Eine Simulation verschiedener Reformen der Rentenversicherung. 1997.

Band 40 Manfred Albring / Eberhard Wille (Hrsg.): Innovationen in der Arzneimitteltherapie. Definition, medizinische Umsetzung und Finanzierung. Bad Orber Gespräche über kontroverse Themen im Gesundheitswesen 25.-27.10.1996. 1997.

Band 41 Eberhard Wille / Manfred Albring (Hrsg.): Reformoptionen im Gesundheitswesen. Bad Orber Gespräche über kontroverse Themen im Gesundheitswesen 7.-8.11.1997. 1998.

Band 42 Manfred Albring / Eberhard Wille (Hrsg.): Szenarien im Gesundheitswesen. Bad Orber Gespräche über kontroverse Themen im Gesundheitswesen 5.-7.11.1998. 1999.

Band 43 Eberhard Wille / Manfred Albring (Hrsg.): Rationalisierungsreserven im deutschen Gesundheitswesen. 2000. 
Band 44 Manfred Albring / Eberhard Wille (Hrsg.): Qualitätsorientierte Vergütungssysteme in der ambulanten und stationären Behandlung. 2001.

Band 45 Martin Pfaff / Dietmar Wassener / Astrid Sterzel / Thomas Neldner: Analyse potentieller Auswirkungen einer Ausweitung des Pharmaversandes in Deutschland. 2002.

Band 46 Eberhard Wille / Manfred Albring (Hrsg.): Konfliktfeld Arzneimittelversorgung. 2002.

Band 47 Udo Schneider: Theorie und Empirie der Arzt-Patient-Beziehung. Zur Anwendung der Principal-Agent-Theorie auf die Gesundheitsnachfrage. 2002.

Band 48 Manfred Albring / Eberhard Wille: Die GKV zwischen Ausgabendynamik, Einnahmenschwäche und Koordinierungsproblemen. 2003.

Band 49 Uwe Jirjahn: X-Ineffizienz, Managementanreize und Produktmarktwettbewerb. 2004.

Band 50 Stefan Resch: Risikoselektion im Mitgliederwettbewerb der Gesetzlichen Krankenversicherung. 2004.

Band 51 Paul Marschall: Lebensstilwandel in Ostdeutschland. Gesundheitsökonomische Implikationen. 2004.

Band 52 Eberhard Wille / Manfred Albring (Hrsg.): Paradigmenwechsel im Gesundheitswesen durch neve Versorgungsstrukturen? 8. Bad Orber Gespräche. 6.-8. November 2003. 2004.

Band 53 Eberhard Wille / Manfred Albring (Hrsg.): Versorgungsstrukturen und Finanzierungsoptionen auf dem Prüfstand. 9. Bad Orber Gespräche. 11.-13. November 2004. 2005.

Band 54 Brit S. Schneider: Gesundheit und Bildung. Theorie und Empirie der Humankapitalinvestitionen. 2007.

Band 55 Klaus Knabner / Eberhard Wille (Hrsg.): Qualität und Nutzen medizinischer Leistungen. 10. Bad Orber Gespräche, 10.-12. November 2005. 2007.

Band 56 Holger Cischinsky: Lebenserwartung, Morbidität und Gesundheitsausgaben. 2007.

Band 57 Eberhard Wille / Klaus Knabner (Hrsg.): Wettbewerb im Gesundheitswesen: Chancen und Grenzen. 11. Bad Orber Gesprăche. 16.-18. November 2006. 2008.

Band 58 Christian lgel: Zur Finanzierung von Kranken- und Pflegeversicherung. Entwicklung, Probleme und Reformmodelle. 2008.

Band 59 Christiane Cischinsky: Auswirkungen der Europäischen Integration auf das deutsche Gesundheitswesen. 2008.

Band 60 Eberhard Wille / Klaus Knabner (Hrsg.): Die besonderen Versorgungsformen: Herausforderungen für Krankenkassen und Leistungserbringer. 12. Bad Orber Gespräche über kontroverse Themen im Gesundheitswesen. 15.-17. November 2007. 2009.

Band 61 Malte Wolff: Interdependenzen von Arzneimittelregulierungen. 2010.

Band 62 Eberhard Wille / Klaus Knabner (Hrsg.): Qualitätssicherung und Patientennutzen. 13. Bad Orber Gespräche über kontroverse Themen im Gesundheitswesen. 20.-21. November 2008. 2010.

Band 63 Eberhard Wille / Klaus Knabner (Hrsg.): Reformkonzepte im Gesundheitswesen nach der Wahl. 14. Bad Orber Gespräche über kontroverse Themen im Gesundheitswesen. 12.-13. November 2009. 2011.

Band 64 Eberhard Wille / Klaus Knabner (Hrsg.): Dezentralisierung und Flexibilisierung im Gesundheitswesen. 15. Bad Orber Gespräche über kontroverse Themen im Gesundheitswesen. 18.-19. November 2010. 2011.

www.peterlang.de 
Eberhard Wille and Klaus Knabner - 978-3-631-75550-1 Downloaded from PubFactory at 01/11/2019 03:39:43AM via free access 
Eberhard Wille and Klaus Knabner - 978-3-631-75550-1 Downloaded from PubFactory at 01/11/2019 03:39:43AM via free access 